\title{
Analysis of Dimensionless Scaling Relationships for Rotating Free Flight Bodies
}

\author{
Shawn A. Smearcheck
}

Follow this and additional works at: https://researchrepository.wvu.edu/etd

\section{Recommended Citation}

Smearcheck, Shawn A., "Analysis of Dimensionless Scaling Relationships for Rotating Free Flight Bodies" (2018). Graduate Theses, Dissertations, and Problem Reports. 6659.

https://researchrepository.wvu.edu/etd/6659

This Thesis is protected by copyright and/or related rights. It has been brought to you by the The Research Repository @ WVU with permission from the rights-holder(s). You are free to use this Thesis in any way that is permitted by the copyright and related rights legislation that applies to your use. For other uses you must obtain permission from the rights-holder(s) directly, unless additional rights are indicated by a Creative Commons license in the record and/ or on the work itself. This Thesis has been accepted for inclusion in WVU Graduate Theses, Dissertations, and Problem Reports collection by an authorized administrator of The Research Repository @ WVU. For more information, please contact researchrepository@mail.wvu.edu. 


\title{
Analysis of Dimensionless Scaling Relationships for Rotating Free Flight Bodies
}

\author{
Shawn A. Smearcheck
}

Thesis submitted to the

Benjamin M. Statler College of Engineering and Mineral Resources

at West Virginia University

in partial fulfillment of the requirements for the degree of

\section{Master of Science}

in

Aerospace Engineering

\section{Wade W. Huebsch, Ph.D., Committee Chairperson Patrick Browning, Ph.D. Christopher D. Griffin, Ph.D.}

Department of Mechanical and Aerospace Engineering

Morgantown, West Virginia

2018

Keywords: Mass Moment of Inertia; Unsteady Aerodynamics; Dimensional analysis; Angular

Velocity; High Speed Analysis; Vicon

Copyright 2018 Shawn A. Smearcheck 


\title{
Abstract \\ Analysis of Dimensionless Scaling Relationships for Rotating Free Flight Bodies
}

\author{
Shawn Smearcheck
}

Though more obscure than traditional flight analysis, the tendency for a rigid body to flutter or tumble in flight during unstable conditions has been analyzed through physical experiments since the 1960s. In more recent years, similar conditions have been subjected to numerical simulation to further understand the effects of dimensionless properties on unstable body motion. It is important to understand dimensionless scaling relationships to compare properties of a body to that of a scaled model or for adjusting data when manufacturing limitations exist. For example, if the geometry of a scaled object could easily be manufactured, within tolerance, but material properties force the mass moment of inertia (MMOI) to deviate from an accepted value, dimensionless scaling relationships could aid in corrections for the desired MMOI. For tumbling bodies, two important properties are the angular velocity and the mass moment of inertia. However, the relationship between dimensionless MMOI and dimensionless angular velocity remains unclear. The purpose of this study was to understand how changing MMOI affects the behavior of angular rates for a rigid body, influenced by repeatable initial conditions and try to characterize that relationship. Using four blocks of varying size and MMOI, a series of tests were conducted to subject the blocks to a range of angular velocities between 0.3 radians $/ \mathrm{sec}$ and $36.5 \mathrm{radians} / \mathrm{sec}$. Body rates were difficult to measure using standard recording devices; therefore, two different high-speed camera systems were used to calculate body rates. Methods: Four different block sizes were manufactured to receive two additional masses. The additional masses were inserted symmetrically about the $x, y$, and $z$ axes of the block. In order to change the MMOI of the block while also preserving its mass, geometry, and density, the additional masses were placed in one of three locations. The first of four different tests involved exposing the blocks to the same initial force at a location that produced rotation about the $z$ axis. Supported uniformly by free jets of air, the blocks traveled horizontally across a flat table. A Photron SA5 was used to record a trajectory at $500 \mathrm{fps}$, and the software Tracker was used to evaluate rates. The second test evaluated body rates as the blocks fell from a height of $243 \mathrm{~cm}$. For each randomized test, the MMOI of the block was changed by adjusting the location of additional masses. Again, using a Photron SA5, high speed video was taken at 500 frames per second (fps) during testing to analyze average linear and angular velocities in the software. The third and fourth tests involved an arm-spring mechanism (i.e. clay pigeon thrower) to induce translational and rotational velocity. However, the third test, just as the first and second, used the software Tracker to evaluate rates. In contrast, the fourth test involved a larger volume over which to track the block's trajectory, and a Vicon camera system was used to evaluate rates. From the high-speed data, a power law scaling relationship between dimensionless MMOI and dimensionless angular velocity was determined. However, the scaling relationship was limited to calculating a velocity ratio rather than individual velocities, due to the translational velocity and rotational velocity being dependent on one another. A two-factor Analysis of Variance (ANOVA) analysis on the data showed that geometric properties of the body greatly influenced the data when compared to the changing MMOI and interaction between the factors. 


\section{Acknowledgments}

I would like to express my gratitude to my family and friends for their constant support and motivation throughout my graduate experience. Especially my parents, Robert and Janet, who have provided me with this opportunity. Without your guidance and involvement in my life, I would not be in the position I am today. I will continue to learn from you both for as long as I can.

To my advisor, Dr. Wade Huebsch, thank you for finding ways to fund my graduate education though teaching assistantships and research. I am grateful to have been a part of the UAV-AJ project; the experience I gained from it will surely benefit me in the future.

To Dr. Christopher Griffin, thank you for offering me the opportunity to do undergraduate research. The position paved the way for me to later start and complete my M.S.A.E. Also, thank you for always taking the time to answer my questions, anytime I knock on your door.

To Dr. Patrick Browning, you have taught me one of the most important lessons I've learned in graduate school: "If things are going well, don't mess with it." It was a good experience learning how to use the pneumatic launcher, and I'm humbled you trusted me to assist you with testing.

To my girlfriend, Marissa Davis, thank you for your patience and guidance throughout the last five years, especially on stressful days.

Finally, to any other persons not specifically mentioned, thank you for your part in making my dream a reality. Whether thorough guidance in class, research, or life, I am grateful for your time and consideration.

Sincerely,

Shawn Smearcheck 


\section{Table of Contents}

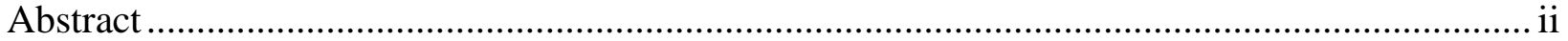

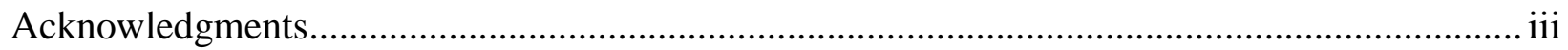

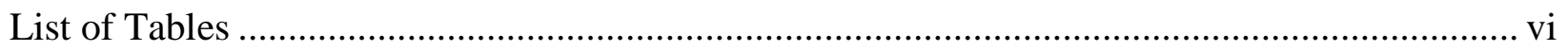

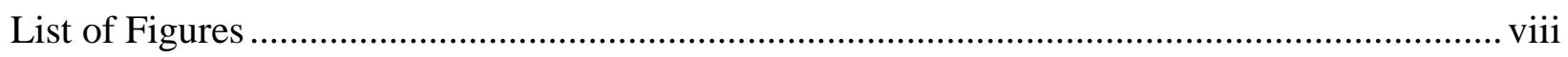

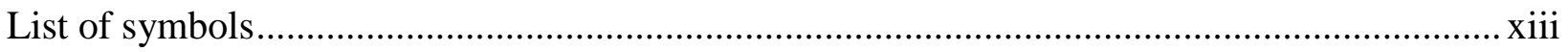

Chapter 1 Problem Statement ……………………………............................................. 1

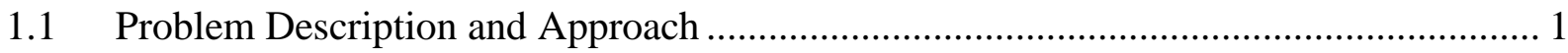

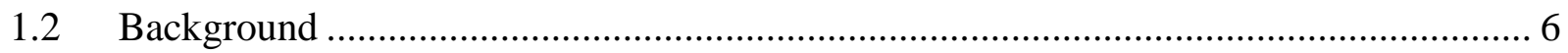

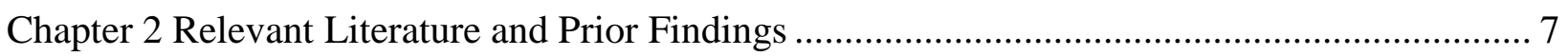

$2.1 \quad$ Unstable and Unsteady Aerodynamics ..................................................................... 7

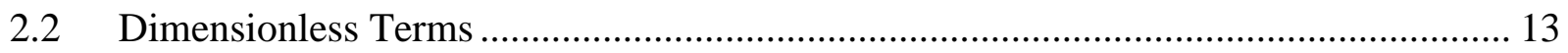

Chapter 3 Block Manufacturing, MMOI Calculations, and Tracking Block .............................. 14

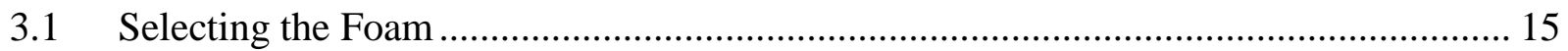

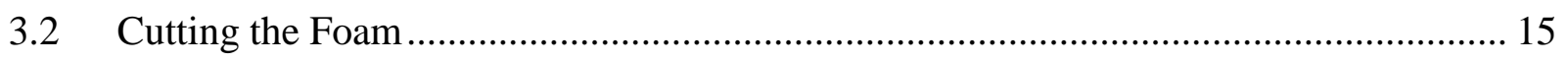

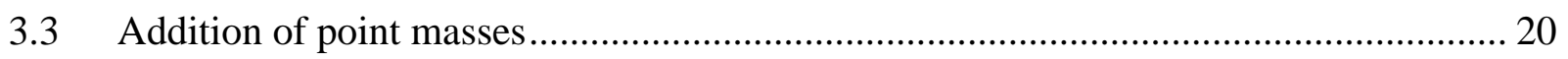

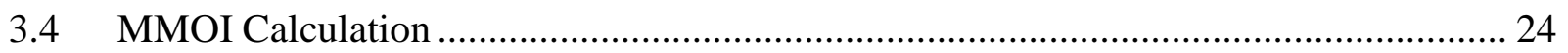

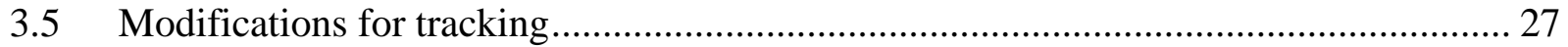

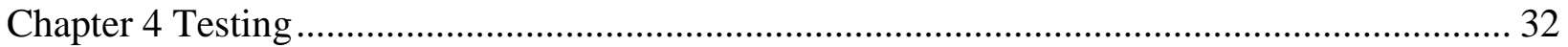

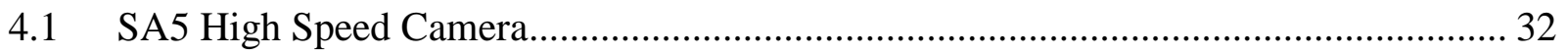

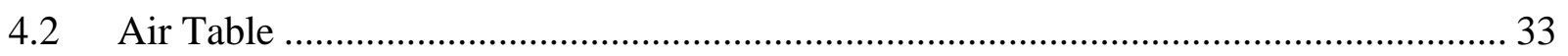

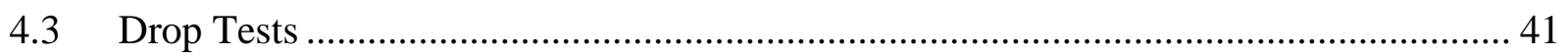

4.4 Throwing Mechanism SA5 High Speed Camera …………………………………....... 46

4.5 Throwing Mechanism VICON Camera System ……………………………….......... 50

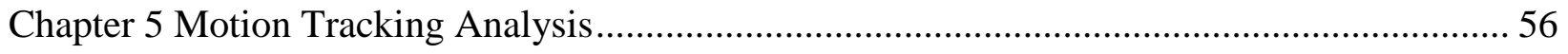

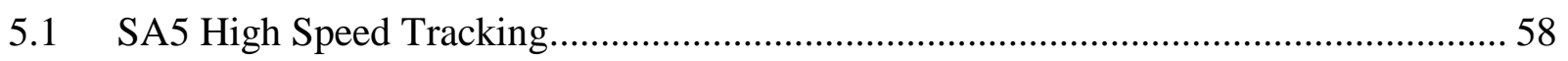

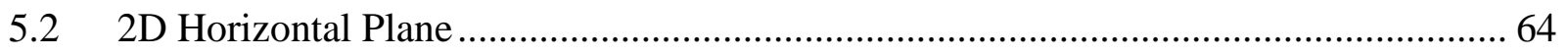

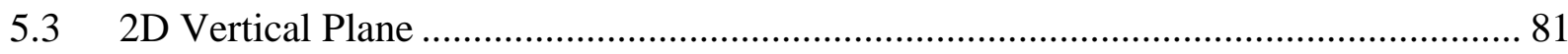

5.4 Throwing Mechanism with Photron SA5 .................................................................. 106

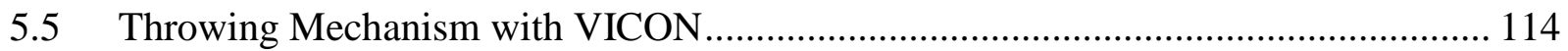

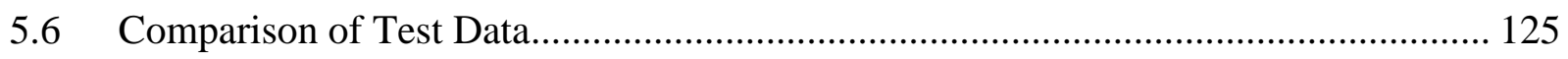

Chapter 6 Conclusions and Recommendations.................................................................... 130 


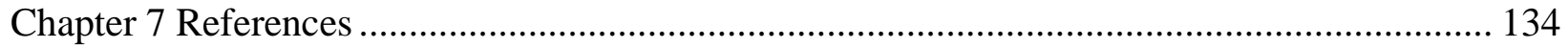

Appendix A

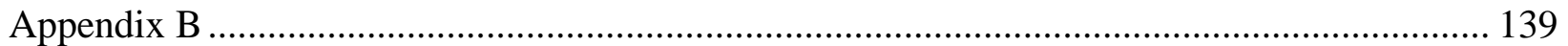




\section{List of Tables}

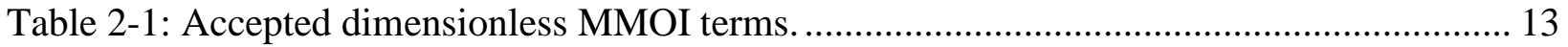

Table 2-2: Length scales used to evaluate scaling relationship between dimensionless terms. ... 14

Table 3-1: Foam block manufacturing repeatability analysis................................................... 19

Table 3-2: Foam block manufacturing repeatability analysis converted to metric units.............. 20

Table 3-3: Maximum allowable mass for each block before interference with table surface. ..... 21

Table 3-4: Additional weight to change MMOI relationship to block mass. ................................ 23

Table 3-5: Numerical MMOI values calculated using Solidworks. ............................................ 26

Table 3-6: Comparison of block length scales to the spherical marker length scale of $10.6 \mathrm{~mm}$

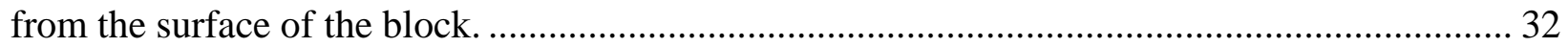

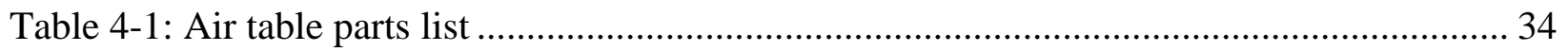

Table 4-2: Impact locations of specimens of different size and MMOI....................................... 44

Table 4-3: Initial thrower arm angle to induce a trajectory trackable by the SA5 camera system.

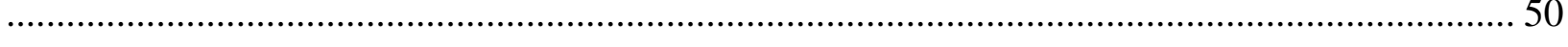

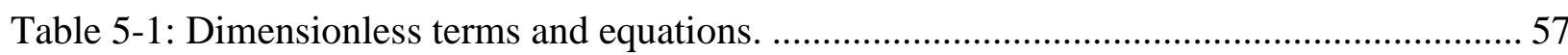

Table 5-2: Length scales analyzed to calculate dimensionless terms. .........................................5 58

Table 5-3: Hammer energy loss due to friction and drag. .............................................................. 71

Table 5-4: Horizontal test block translational velocities collected from Tracker software and

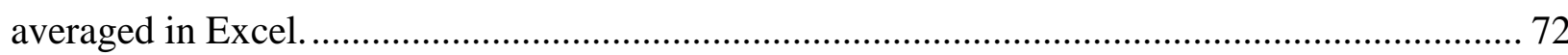

Table 5-5: Horizontal test block rotational velocities collected from Tracker software and averaged in Excel................................................................................................................ 72

Table 5-6: Translational and rotational velocities used for dimensionless term calculations. Average of values over three runs. .................................................................................... 73

Table 5-7: Trendline equations from Figure 5.20. Equations used to interpolate more MMOI values to compare to experimental data..................................................................................... 77 Table 5-8: Interpolated values for comparison of theoretical and experimental data using Block 1

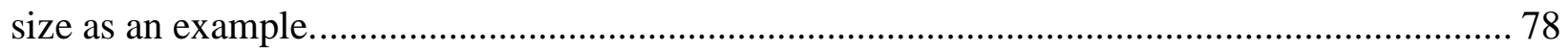

Table 5-9: ANOVA analysis of translational velocity data from Table 5-4 ................................ 80

Table 5-10: ANOVA analysis of rotational velocity data from Table 5-5 .................................... 81

Table 5-11: Methods tested to determine an accurate representation of Ux_avearage for drop

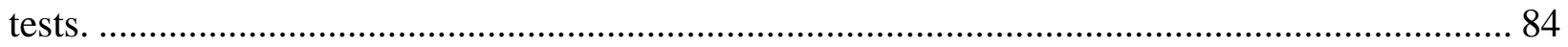

Table 5-12: Average translational velocities calculated using the three methods shown as

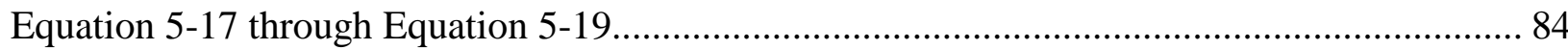

Table 5-13: Drop test measured data used for analysis. ............................................................... 86

Table 5-14: Block properties for theoretical calculations based on initial conditions.................. 98

Table 5-15: Tracker data and additional properties used for dimensionless term calculations.... 99

Table 5-16: Drop test block velocities collected for ANOVA analysis........................................ 101

Table 5-17: ANOVA calculations of MMOI and size effects on translational velocity ............. 101

Table 5-18: Drop test block angular velocities collected for ANOVA analysis.......................... 102

Table 5-19: ANOVA calculations of MMOI and size effects on angular velocity.................... 102

Table 5-20: Drop test block landing locations collected for ANOVA analysis. ......................... 104

Table 5-21: ANOVA calculations of MMOI and size effects on landing location. ..................... 104

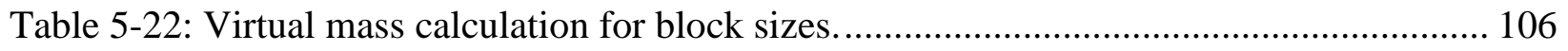


Table 5-23: Throwing arm average angular velocity data..... 109

Table 5-24: Average translational velocity data collected from each run for ANOVA analysis.

Table 5-25: Average angular velocity data collected from each run for ANOVA analysis....... 113

Table 5-26: ANOVA data based on translational velocity data. ........................................... 114

Table 5-27: ANOVA data based on rotational velocity data................................................... 114

Table 5-28: Average velocity data calculated from smoothed data collected by Vicon system. 117

Table 5-29: Tracker data for throwing arm angular velocity over three runs.......................... 120

Table 5-30: Vicon translational velocity data used for ANOVA analysis.............................. 124

Table 5-31: Vicon rotational velocity data used for ANOVA analysis. Block 4 omitted from

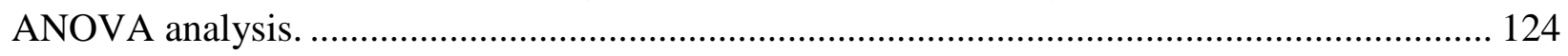

Table 5-32: ANOVA analysis for Ux from Vicon data..................................................... 125

Table 5-33: ANOVA analysis for $\omega$ from Vicon data........................................................ 125

Table 5-34: Combined ANOVA analysis for Ux for each of the four testing methods. ........... 129

Table 5-35: Combined ANOVA analysis for $\omega$ for each of the four testing methods. ............. 129 


\section{List of Figures}

Figure 1.1: Block axes and adjustable point mass locations for changing MMOI....................... 2

Figure 1.2: Air table and camera mount for horizontal plane in testing method one. .................. 3

Figure 1.3: Drop mechanism experimental set up for testing method two................................ 4

Figure 1.4: Clay thrower experimental set up for analysis with SA5 high speed camera in testing method three.

Figure 1.5: Vicon camera system experimental setup used for trajectory analysis in testing method four.....

Figure 2.1: Trajectories obtained from direct numerical simulations of the two-dimensional Navier-Stokes equation for freely falling plates in the transition range between fluttering and tumbling: (a) periodic fluttering with the non-dimensional period of oscillation $\mathrm{T}=8.1$, (b) fluttering close to the transition between fluttering and tumbling with $\mathrm{T}=17.3$, (c) mixture of fluttering and tumbling with $\mathrm{T}=34.1$ for $\beta=1 / 12$, and $(\mathrm{d})$ periodic tumbling with $\mathrm{T}=12.1$ [7]. 8 Figure 2.2: Falling trajectory of a rectangle from fluttering to tumbling motion [8].

Figure 2.3: (a) View normal to plane of staggered arrangement of two rows of equally spaced (5 $\mathrm{cm}$ ) vortex rings left in the wake $40 \mathrm{sec}$ after passage of disk which exhibited oscillatory pitching motion as it fell almost vertically, $\mathrm{Re}=170$. (b) Wake of a freely falling disk which exhibited regular translational and pitching oscillations, $\mathrm{Re}=1547$ [9]....................................... 11 Figure 2.4: Measured plate trajectories: (a) the fluttering plate with $\mathrm{h}=0.081 \mathrm{~cm}$ and $\beta=1 / 14$, (b) two apparently chaotic trajectories for the $\mathrm{h}=0.162 \mathrm{~cm}$ and $\beta=1 / 6$ plate, and (c) the tumbling plate with $h=0.162 \mathrm{~cm}$ and $\beta=1 / 5$. For clarity the two trajectories in (b) are displaced from each other $0.5 \mathrm{~cm}$ horizontally and $1.5 \mathrm{~cm}$ vertically. The thick line segments show the plate cross-sections with time intervals of $0.04 \mathrm{sec}$ in (a) and $0.025 \mathrm{sec}$ in (b) and (c) [6]......... 12

Figure 3.1: Hot-wire used for cutting foam ................................................................... 15

Figure 3.2: CAD model showing wooden jig used to cut foam............................................. 16

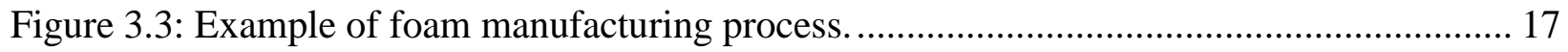

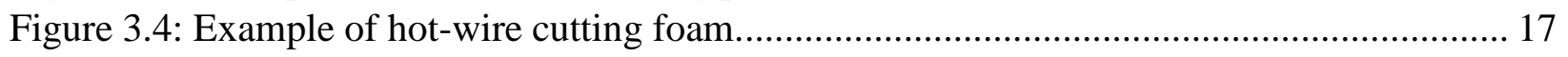

Figure 3.5: Weighing technique for foam blocks and additional masses. Foam measured in ounces and then converted to grams while bolts measured in grams initially. 18

Figure 3.6: Block measurement techniques. (a) Height measurement taken using digital micrometer. (b) Length measurement taken using tape measure. (c) Width measurement taken using tape measure.

Figure 3.7: Additional weights for changing MMOI of block. (a) Additional weights designed for Block 1. (b) Additional weights designed for Block 2. (c) Additional weights designed for Block 3. (d) Additional weights designed for Block 4. ......................................................... 22

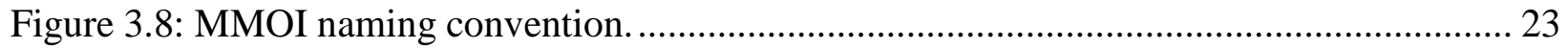

Figure 3.9: Covering holes on blocks to create even surface plane......................................... 24

Figure 3.10: Solidworks assembly of Block 1 - MMOI 1 with mass properties window showing calculations. 25

Figure 3.11: Enhanced view of mass properties window and MMOI calculation, shown in red box, for specific configuration of block and additional weights. 
Figure 3.12: Location of high contrast stickers for tracking. The center sticker was used to track translational velocity with respect to a user defined coordinate system while the sticker in the upper left corner was used to track rotational velocity with respect to the center sticker. 27 Figure 3.13: Demonstration of reflective property of markers for analysis using Vicon camera system. (a) Picture taken using no flash. (b) Picture taken using flash. 28 Figure 3.14: (a) Face one of Block 2, which shows three spherical markers indicated by red arrows. (b) Face two of Block 2 which shows two additional spherical markers indicated by red arrows. A total of five spherical markers were used on each block size.

Figure 3.15: Object coordinate system for throwing mechanism evaluated with Vicon camera system.

Figure 3.16: Object created in Vicon software based on reflective markers. Coordinate system at CG oriented to match global coordinate system.

Figure 3.17: Spherical marker length scale of $10.6 \mathrm{~mm}$ from the bottom of the base plate to the

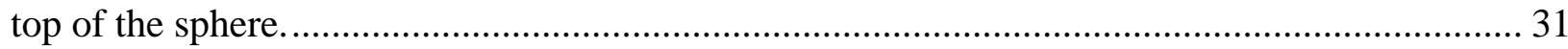

Figure 4.1: Air table, firing mechanism, and Unistrut camera mount. .................................... 33

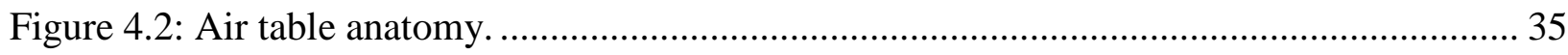

Figure 4.3: Fan used to pressurize the table........................................................................ 36

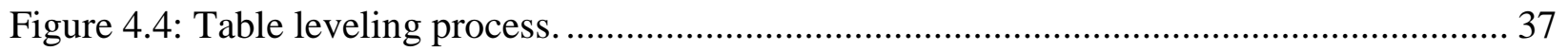

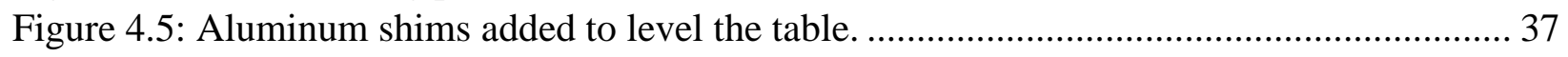

Figure 4.6: CAD model of SA5 high speed camera mount for angling the camera 90 degrees to

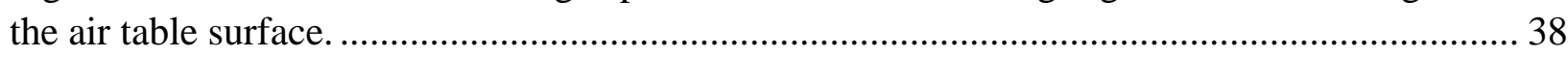

Figure 4.7: Camera mount for horizontal plane experiments. ................................................ 39 Figure 4.8: Camera mounted above air table during horizontal tests. (a) Side view. (b) Rear view.

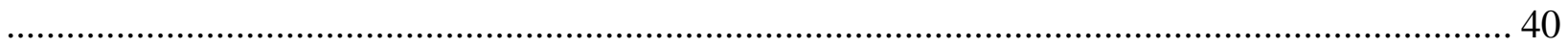

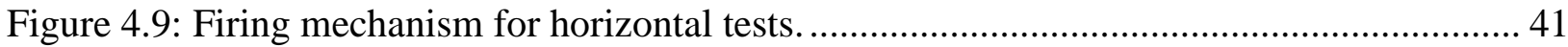
Figure 4.10: CAD and dimensions of drop mechanism experiment. Dimensions are shown in

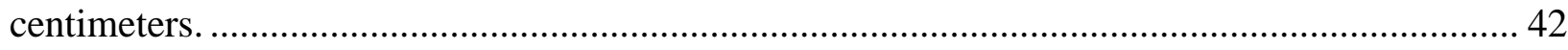

Figure 4.11: Drop test experiment setup to determine relationship between a relationship between dimensionless MMOI and angular velocity and tendency to tumble into perpendicular

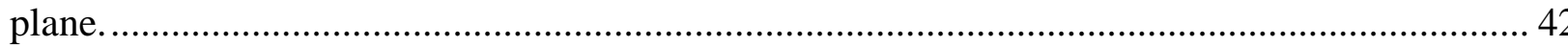

Figure 4.12: Initial condition of block for drop testing. The block is held by two 1/4" rods until one is quickly removed using the attached string. ............................................................ 43 Figure 4.13: Grid of 4"x4" squares used to determine horizontal block motion. The center of the grid, signified using a block dot, was aligned with the $\mathrm{CG}$ of the block usign a plumb bob. ..... 44 Figure 4.14: Visual representation of the impact locations of test articles. Numbers represent MMOI of the block while shape and color of represent size. (E.g. a blue triangle containing the number 1 represents the Block 1 with MMOI 1).

Figure 4.15: (a) GoPro perspective used to determine block location at impact in the horizontal plane. (b) High speed camera perspective used to determine block location at impact in the vertical plane. Block 2 with MMOI 1 is used as an example. The location for this example is G5 and can cross referenced in Figure 4.14, showing all drop locations. 46 Figure 4.16: Clay pigeon thrower used to induce rotational and translational motion. Note that the midpoint of the block in the "L" dimension is aligned with the end of the holder. 
Figure 4.17: Throwing arm test experimental setup. Dimensions given in inches.................... 48

4.18: Initial thrower arm calculation using level and protractor......................................... 49

Figure 4.19: Throwing mechanism angle adjustment plate locking system. The plates were

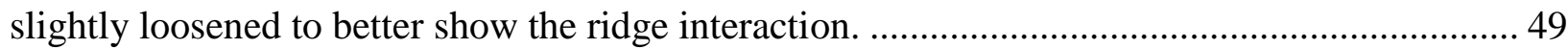

Figure 4.20: CAD model of Vicon test volume and camera configuration.............................. 50

Figure 4.21: Vicon camera system experimental setup with coordinate system overlaid........... 51

Figure 4.22: Vicon masking process to remove reflective material from the volume. Blue marks indicate reflective material such as other cameras, metal, etc. that has been masked................. 52 Figure 4.23: Vicon active wand, with coordinate system overlaid, used for system calibration.. 53 Figure 4.24: Setting the global origin for the Vicon system, using the active wand.................. 53 Figure 4.25: Comparison of block placement for Block 1 and Block 3 in the throwing mechanism. The blocks were placed so that only half of the L dimension was in contact with the mechanism. The yellow line indicates the centerline of the block as it extends through the tip of

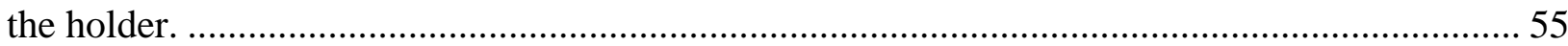

Figure 4.26: Initial thrower arm calculation using level and protractor. ................................. 55

Figure 5.1: Grid of 4 inch squares used to calibrate images to reduce edge distortion. .............. 59

Figure 5.2: Mean reprojection error per image calculated by MATLAB Camera Calibration App.

An overall mean error of less than 1 pixel was used to calibrate images.

Figure 5.3: MATLAB extrinsic projection of camera location in relation to calibration board

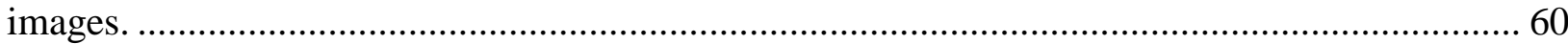

Figure 5.4: Comparison of original image, (a), and calibrated image, (b) . ............................. 61

Figure 5.5: Tracking a stationary point to determine noise in the software. ........................... 62

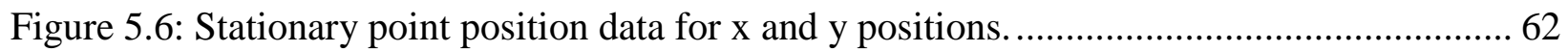

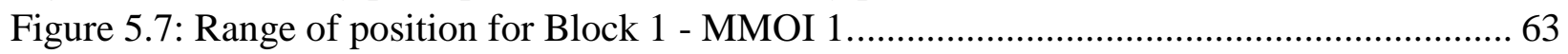

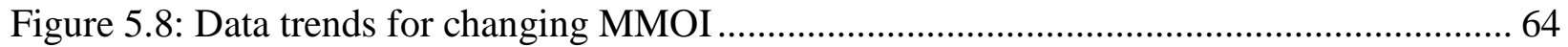

Figure 5.9: Default Tracker coordinate system (blue) and trail of tracked velocity (red)........... 65

Figure 5.10: (a) Range of velocity variation over three runs for Block 1. (b) Range of velocity variation over three runs for Block 2. (c) Range of velocity variation over three runs for Block 3.

Figure 5.11: Calculating hammer impact velocity using conservation of energy in two stages.

Stage 1 indicates initial height of the hammer. Stage 2 indicates the position of the hammer just

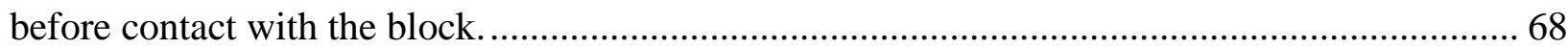

Figure 5.12: Impact location on the block during contact from hammer. ............................... 68

Figure 5.13: Experimental hammer velocity to determine loss due to friction and drag. One data point was taken for 15 runs and averaged together to determine hammer velocity. .................. 71

Figure 5.14: Length scale of hydraulic diameter ............................................................ 74

Figure 5.15: Length scale of surface area divided by L.................................................. 74

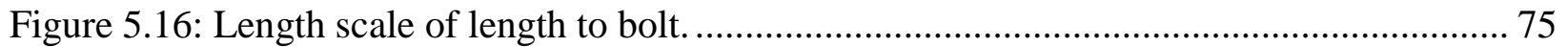

Figure 5.17: Length scale of dimension $\mathrm{W}$....................................................................... 75

Figure 5.18: Length scale of volume divided by total surface area......................................... 76

Figure 5.19: Length scale of dimension $\mathrm{W}$ multiplied by aspect ratio..................................... 76

Figure 5.20: Curve fit equation to interpolate more MMOI data points. Data is used to compare experiential data to theoretical. (a) Block 1. (b) Block 2. (c) Block 3. (d) Block 4.................... 77 
Figure 5.21: Semi-log plot comparing experimental and theoretical data for dimensionless MMOI and dimensionless angular velocity of block. 79

Figure 5.22: Demonstration of Tracker's tape measure tool (indicated by the red arrow) to determine a distance, $\mathrm{x}$, for calculating average velocity. 83

Figure 5.23: Block rotating point of interest out of view. Tracker software unable to determine

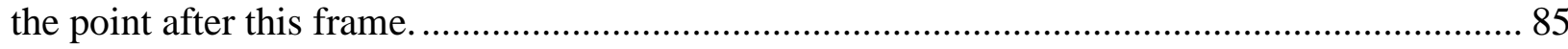
Figure 5.24: Hydraulic Diameter length scale using Equation 5-17 average velocity values. ..... 86 Figure 5.25: Semi-Log plot of SA/L length scale using Equation 5-17 average velocity values. 87 Figure 5.26: Length to bolt length scale using Equation 5-17 average velocity values. ............. 87 Figure 5.27: Largest dimension, W, length scale using Equation 5-17 average velocity values.. 88 Figure 5.28: Volume/SA length scale using Equation 5-17 average velocity values................. 88 Figure 5.29: Largest dimension, W, multiplied by aspect ratio length scale using Equation 5-17 average velocity values. 89

Figure 5.30: Hydraulic diameter length scale using Equation 5-18 average velocity values....... 89 Figure 5.31:Semi-Log plot of SA/L length scale using Equation 5-18 average velocity values.. 90 Figure 5.32: Length to bolt length scale using Equation 5-18 average velocity values. ............. 90 Figure 5.33: Largest dimension, W, length scale using Equation 5-18 average velocity values.. 91 Figure 5.34: Volume/SA length scale using Equation 5-18 average velocity values. 91 Figure 5.35: Largest dimension, W, multiplied by aspect ratio length scale using Equation 5-18

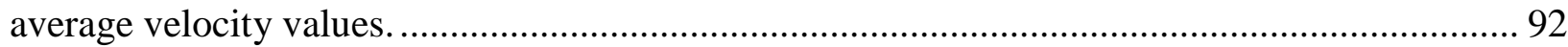
Figure 5.36: Hydraulic diameter length scale using Equation 5-19 average velocity values...... 92 Figure 5.37: Semi-Log plot of SA/L length scale using Equation 5-19 average velocity values. 93 Figure 5.38: Length to bolt length scale using Equation 5-19 average velocity values. ............. 93 Figure 5.39: Largest dimension, W, length scale using Equation 5-19 average velocity values.. 94 Figure 5.40: Volume/SA length scale using Equation 5-19 average velocity values................. 94 Figure 5.41: Largest dimension, W, multiplied by aspect ratio length scale using Equation 5-19

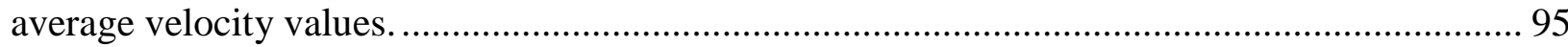

Figure 5.42: Initial condition of the blocks for drop testing. ........................................... 96 Figure 5.43: Experimental dimensionless terms versus theoretical dimensionless terms for the length scale of hydraulic diameter, using the velocity calculations based off of Equation 5-18.100 Figure 5.44: Experimental dimensionless terms versus theoretical dimensionless terms for the length scale of SA/L, using the velocity calculations based off of Equation 5-18 ................. 100 Figure 5.45: Assigned coordinate values for drop coordinates for ANOVA analysis. Where the

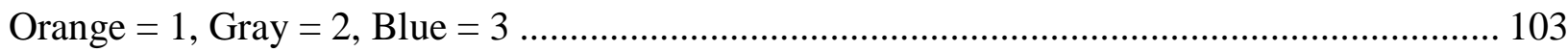

Figure 5.46: Froude number calculation for drop tests................................................. 105 Figure 5.47: User defined reference coordinate system, placed at the hinge point of the throwing mechanism. Additionally, the projected paths of the points of interest and user defined scale. 107 Figure 5.48: Tracking throwing ar angular velocity for SA5 tests to compare with Vicon tests.

Figure 5.49: Dimensionless term comparison based on hydraulic diameter length scale......... 109

Figure 5.50: Dimensionless term comparison based on SA/L length scale............................ 110

Figure 5.51: Dimensionless term comparison based on distant to bolt length scale. ............... 110

Figure 5.52: Dimensionless term comparison based on largest dimension, W, length scale..... 111 
Figure 5.53: Dimensionless term comparison based on Volume/SA length scale.

Figure 5.54: Dimensionless term comparison based on largest dimension, W, multiplied by aspect ratio length scale.

Figure 5.55: Object in launcher before motion was induced.

Figure 5.56: Translational velocity of the block vs. smoothed data to demonstrate noise in

measurement.

Figure 5.57: Excessive noise in run 1 of Block 4 for MMOI 1.

Figure 5.58: Raw translational data for Block 1- Run 1- MMOI 1 taken from Vicon system.

Only Downrange Velocity in the $\mathrm{x}$-axis was analyzed.

Figure 5.59: Raw rotational rate data for Block 1- Run 1- MMOI 1 taken from Vicon system.

Only pitching rate about the y-axis was desired.

Figure 5.60: Hydraulic diameter length scale for Vicon high speed data.............................. 121

Figure 5.61: SA/L length scale for Vicon high speed data................................................. 121

Figure 5.62: Distance to bolt length scale for Vicon high speed data. ...................................... 122

Figure 5.63: Dimension W length scale for Vicon high speed data. ...................................... 122

Figure 5.64: Volume/SA length scale for Vicon high speed data....................................... 123

Figure 5.65: Dimension W multiplied by aspect ratio length scale for Vicon high speed data.. 123 Figure 5.66: Dimensionless term data for the four test methods calculated using the SA/L length scale. Three of the four methods tend to converge to a single line. Demonstrating a scaling relationship. 127

Figure 5.67: Dimensionless data for the horizontal plane test, thrower test using Sa5, and thrower test using Vicon with a power function trendline overlaid. 127

Figure 6.1: Application Example 
List of symbols

\begin{tabular}{|c|c|c|}
\hline Symbol & Description & Units \\
\hline$I$ & mass moment of inertia & $\mathrm{g}-\mathrm{cm}^{2}$ \\
\hline$L$ & length of the block & $\mathrm{cm}$ \\
\hline $\mathrm{W}$ & width of the block & $\mathrm{cm}$ \\
\hline $\mathrm{H}$ & height of the block & $\mathrm{cm}$ \\
\hline$\omega$ & angular velocity & $\mathrm{rad} / \mathrm{sec}$ \\
\hline$I^{*}$ & dimensionless mass moment of inertia & - \\
\hline$\omega^{*}$ & dimensionless angular velocity & - \\
\hline$\rho_{f}$ & density of fluid & $\mathrm{g} / \mathrm{cm}^{3}$ \\
\hline$\rho_{b}$ & density of block & $\mathrm{g} / \mathrm{cm}^{3}$ \\
\hline$g$ & acceleration due to gravity & $\mathrm{cm} / \mathrm{sec}^{2}$ \\
\hline$a$ & acceleration & $\mathrm{cm} / \mathrm{sec}^{2}$ \\
\hline$F$ & force & $\mathrm{N}$ \\
\hline$m_{h}$ & mass of hammer & $\mathrm{g}$ \\
\hline$m_{b}$ & mass of block & $\mathrm{g}$ \\
\hline$I_{z z}$ & mass moment of inertia about the $\mathrm{z}$-axis & $\mathrm{g}-\mathrm{cm}^{2}$ \\
\hline$x$ & $\mathrm{x}$-axis & - \\
\hline$y$ & $y$-axis & - \\
\hline$z$ & $\mathrm{z}$-axis & - \\
\hline fps & frames per second & frames/sec \\
\hline$C G$ & center of gravity & - \\
\hline$P$ & $\begin{array}{l}\text { perpendicular distance to centerline of impact for } \\
\text { eccentric collision }\end{array}$ & $\mathrm{cm}$ \\
\hline$u_{h}$ & hammer velocity at impact & $\mathrm{cm} / \mathrm{sec}$ \\
\hline$u_{b}$ & initial block velocity & $\mathrm{cm} / \mathrm{sec}$ \\
\hline$v_{h}$ & hammer velocity after impact & $\mathrm{cm} / \mathrm{sec}$ \\
\hline$v_{b}$ & block tangential velocity after impact & $\mathrm{cm} / \mathrm{sec}$ \\
\hline$C M$ & center of mass & - \\
\hline$H P$ & horse power & $\mathrm{ft}-\mathrm{lb} / \mathrm{sec}$ \\
\hline $\mathrm{Hz}$ & Hertz & $1 / \mathrm{sec}$ \\
\hline
\end{tabular}




\section{Chapter 1 Problem Statement}

\subsection{Problem Description and Approach}

To better represent real world applications, an understanding of scaling relationships between dimensionless terms is essential to understanding important dimensionless groupings, predicting behavior of different scales, and to develop a basis for which data can be corrected. Furthermore, the scaling effects of mass moment of inertia (MMOI) for a rigid body on angular rates are particularly important for unstable bodies. The purpose of this study was to develop a method for comparing MMOI of a body to its angular velocity to aid in trajectory analysis. For example, if a scaled rigid body of interest were able to meet geometric criteria but material properties alter MMOI, a correction to the data could be calculated to better represent the rates of the body with the adjusted MMOI.

Dimensionless mass moment of inertia, $I^{*}$, and dimensionless angular velocity, $\omega^{*}$, were analyzed to determine effects on a simple geometric body for application to flight stability of an Unmanned Aerial System (UAS). High speed video was used to capture the flight pattern of the specimens. Four foam blocks of different sizes, Block 1-4, were tested with three possible MMOIs. The four distinct sizes were used to determine how geometric properties affect the body rates. The dimensions were determined based on manufacturing capabilities, material availability, and creating a range of distinct MMOI values. To change MMOI of the blocks, additional weights were added to one of three locations symmetric about the $x, y$, and $z$ axes. The three locations for weights were selected to be $0.125 \mathrm{~W}$, where $W$ represents the largest dimension of the block. Using the scale of $0.125 \mathrm{~W}$ allowed the additional weights to be spaced evenly across the blocks. That is, the spacing between the weight locations was the same. Bolts weighting approximately the same weight as the block were added to create a significant change in the MMOI. Selecting additional weights with the same mass as the blocks was chosen arbitrarily as a simple means to ensure the MMOI would change significantly enough to affect the data. An example of block dimensions and additional weight adjustment locations is shown as Figure 1.1. 


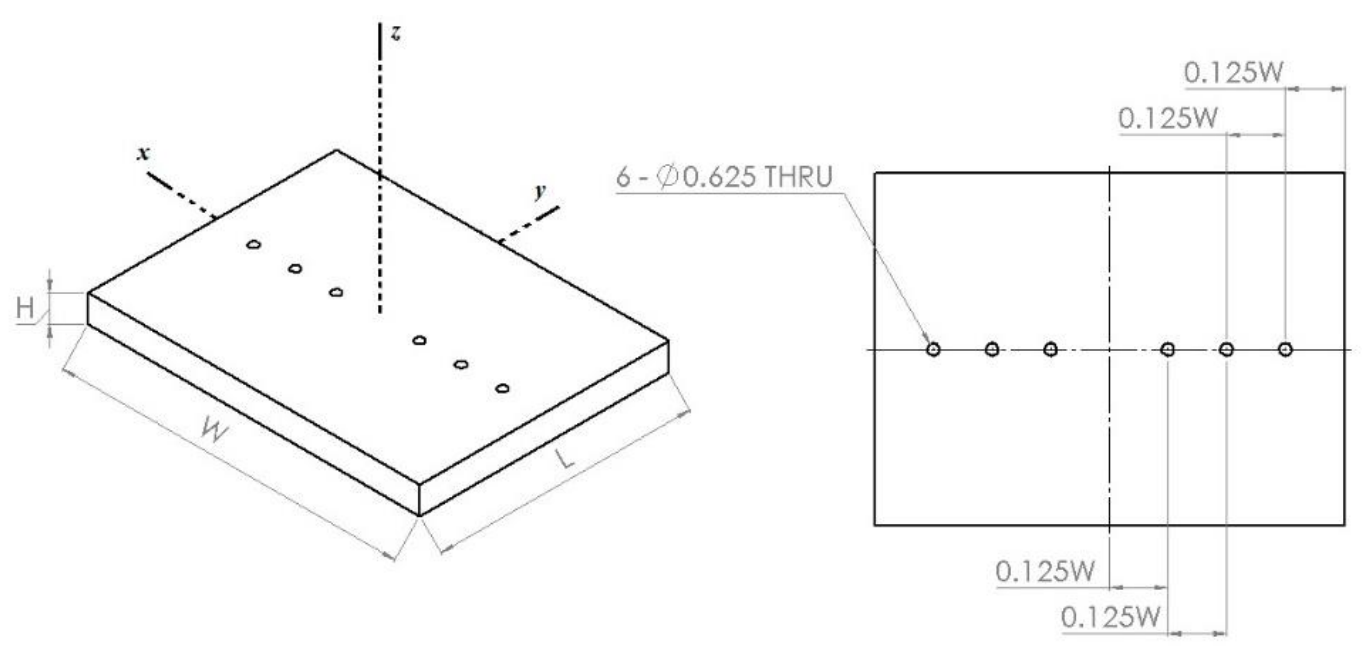

Figure 1.1: Block axes and adjustable point mass locations for changing MMOI.

Four approaches were observed to determine the effects of mass moment of inertia and angular velocity on the trajectory of a rigid body. For each method of testing, three runs at each MMOI were evaluated for a total of 36 runs. The rationale for selecting three runs is further explained in Chapter 5, but, simply, it was based on the repeatability of block trajectory. First, using a pendulum with known initial energy, the blocks were subjected to an impact at a consistent location from the CG. The impact induced an initial translational velocity and angular velocity in the block. As the block translated across the horizontal surface, free jets of air counteracted the blocks weight, allowing it to hover just above the table's surface. Using air to support the block during its trajectory reduced loss due to friction as well as maintained motion in a single plane. A Photron SA5 was mounted above the table to capture a two-dimensional view of each trajectory at $500 \mathrm{fps}$. The high-speed video was then analyzed in the software Tracker to determine rates. The experimental setup for the first method of testing is shown in Figure 1.2. More detail is provided in Chapter 4. 


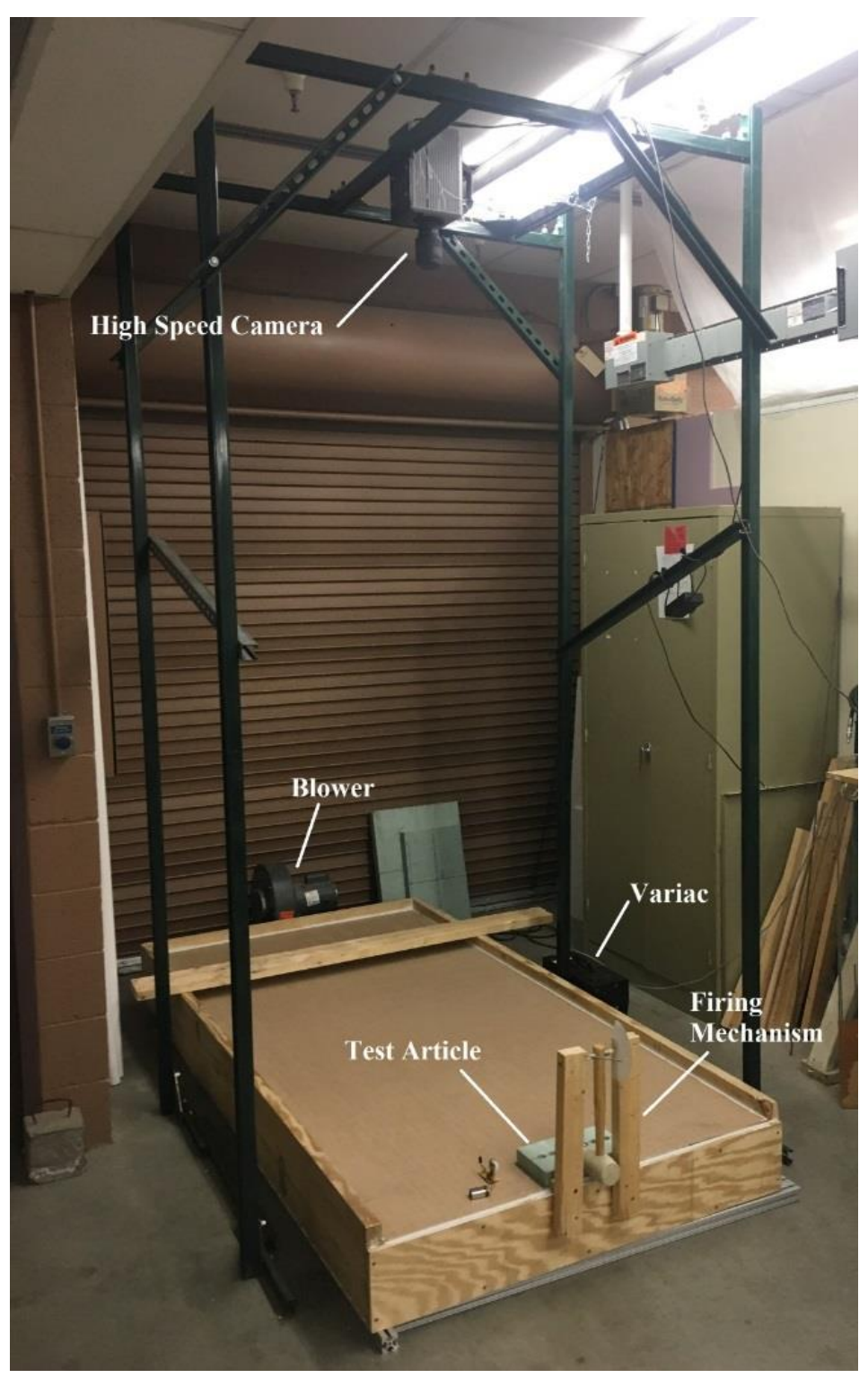

Figure 1.2: Air table and camera mount for horizontal plane in testing method one.

Subsequently, the same blocks were to be dropped in a vertical plane, as shown in Figure 1.3. In this testing, instead of inducing motion with kinetic energy from an outside mass, the blocks were forced into motion due to the effects of gravity. The blocks were placed in a holding mechanism at a slight angle. Two pins supported each end of the block, one fixed and the other removable. Using a pull string attached to the support pin, the pin could quickly be ejected from its slot in the same way a table cloth is pulled out from under a set of dishes. As in the first testing method, the Photron SA5 camera and Tracker software were used in conjunction to calculate rates of the blocks for each run. 


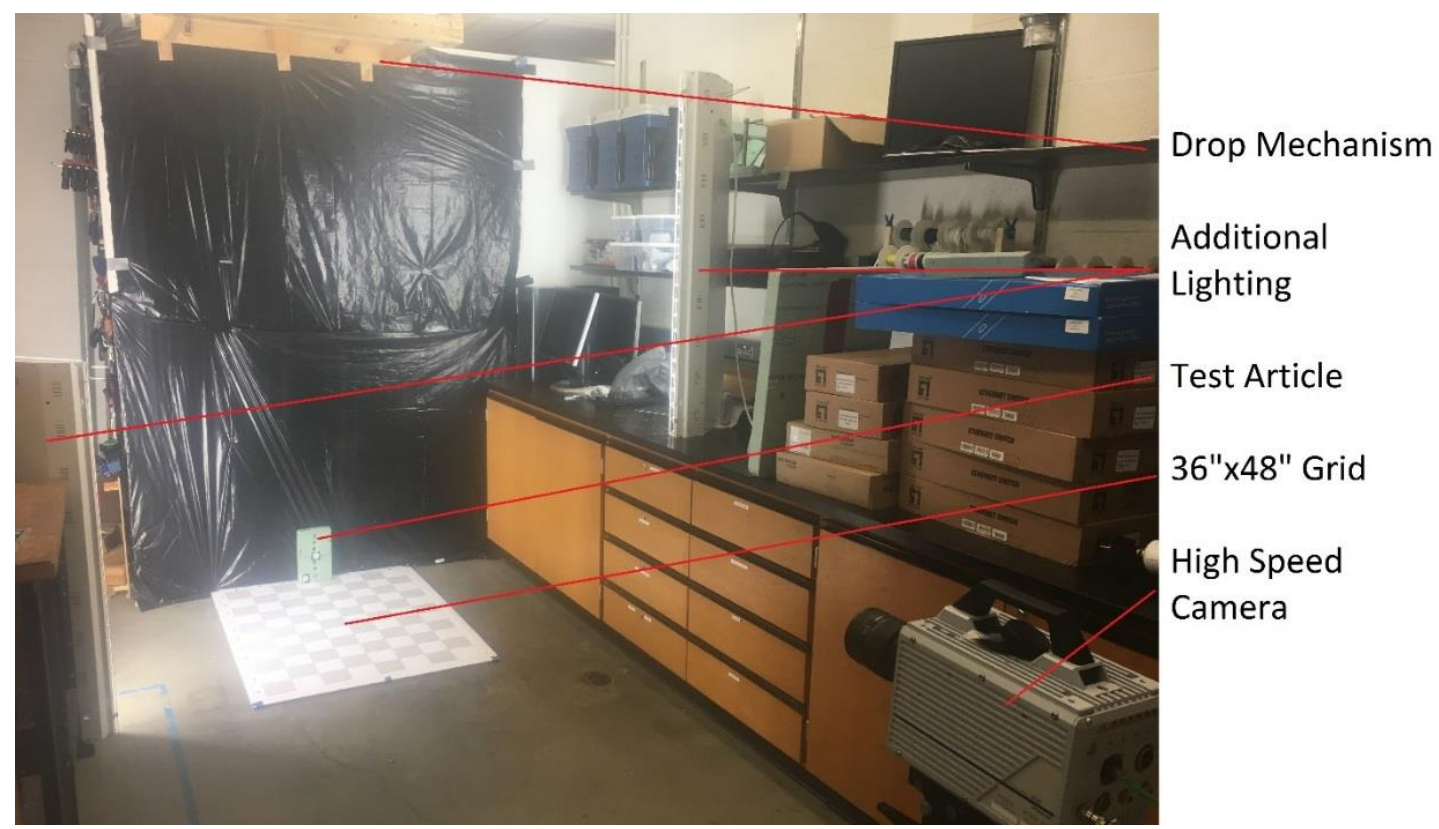

Figure 1.3: Drop mechanism experimental set up for testing method two.

The third method involved use of a sporting clay thrower to induce motion (Figure 1.4). The clay thrower transferred enough energy to the blocks to reach an angular velocity of approximately 2000 degrees per second, the highest of the three methods to induce motion. The thrower tests were analyzed using the Photron SA5 high speed camera and Tracker, as in the horizontal table and drop tests. Figure 1.4 shows the experimental setup for the third test method. To keep the blocks from becoming damaged, a damping screen was used to cushion the blocks as they decelerated. Careful consideration was also given to placing the blocks at a consistent location on the launcher.

Additionally, to compare results between two systems, the clay thrower tests were repeated and analyzed using a Vicon high speed tracking system (Figure 1.5). For this method, 12 Vicon cameras were used to capture the block trajectory at 500fps. The blocks were outfitted with highly reflective markers to allow the software to create an object. The Vicon system used infrared (IR) light to distinguish between spherical markers and noise in the volume. This was the only method that had the capability to track the block's trajectory in 3D. Ideally, since only rotation about the $z$-axis was desired, the rates about the $x$ and $y$ axes were kept as low as possible. The throwing mechanism itself was also tracked to determine the maximum velocity as it released the blocks. Additional testing details are provided in Chapter 4. 


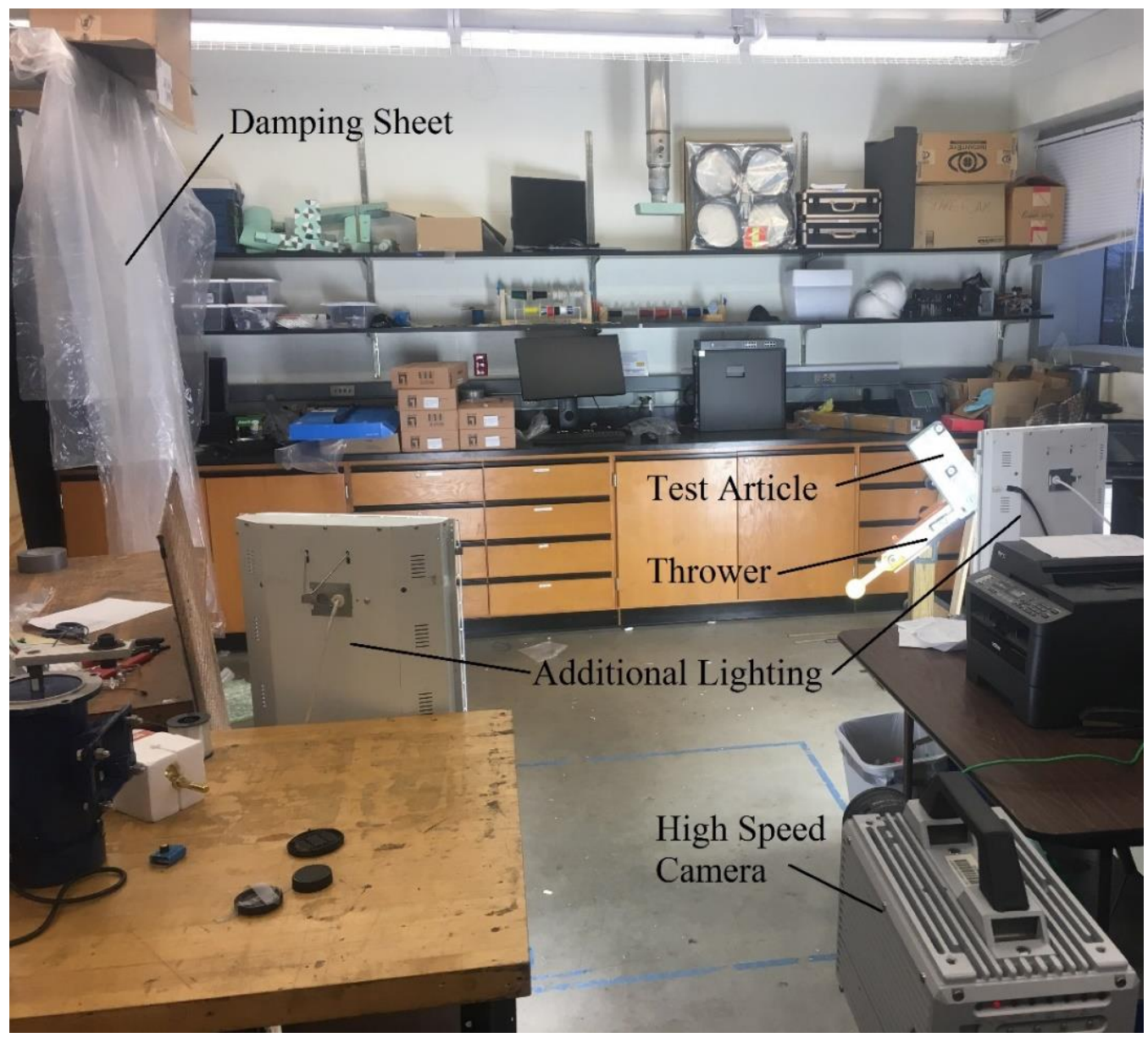

Figure 1.4: Clay thrower experimental set up for analysis with SA5 high speed camera in testing method three. 


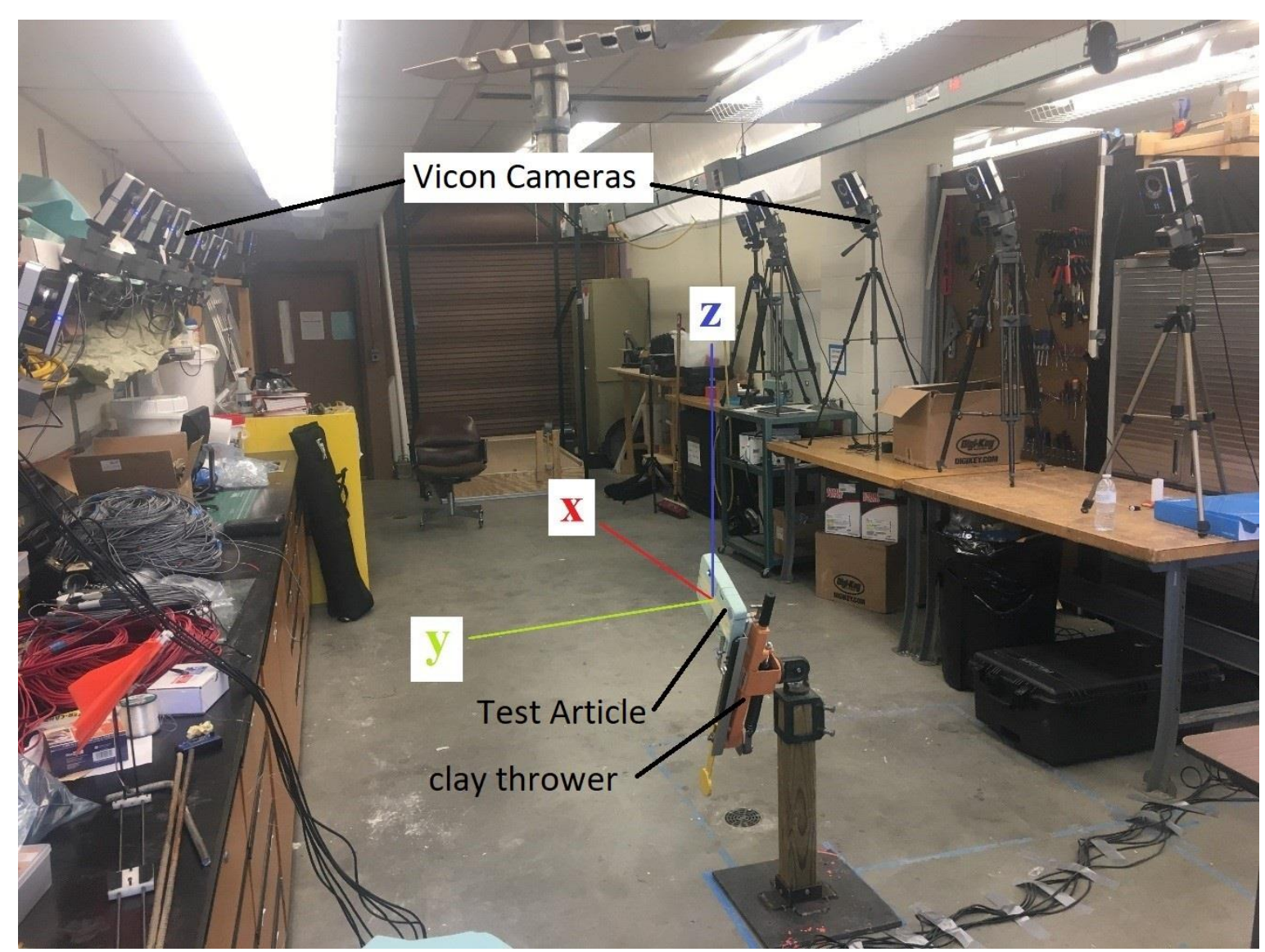

Figure 1.5: Vicon camera system experimental setup used for trajectory analysis in testing method four.

\subsection{Background}

The study of aerodynamics can be traced back to the late $15^{\text {th }}$ century when inventor Leonardo da Vinci began to theorize about flying machines [1]. Da Vinci used his knowledge of flying animals in nature, as well as mythology, to design numerous methods of controlled flight [1].

In more recent years, there has been a higher inclination to understand the science of unstable aerodynamics. For the purposes of this study, unstable aerodynamics was defined to be rapid rotation throughout a high range of pitch angles. Unstable flow phenomena can be analyzed in nature as objects, such as leaves, cards, paper, or other materials with unconventional aerodynamic geometries, fall through the air. For application purposes, unstable aerodynamics is used to determine how a body will react under specific boundary conditions. For example, studies performed on the unstable aerodynamics involved with aircraft seat ejection analyzed important factors such as canopy detachment, seat performance, and body motion during ejection [2, 3, 4]. 
Unstable and unsteady aerodynamics have also aided in the advancement of UASs through means of trajectory analysis and prediction in various mediums [5]. Using numerical methods to simulate unstable trajectories can greatly reduce the cost of UAS design and analysis. However, with more complex fuselage designs and the combination of detachable payloads, small changes to the boundary conditions can greatly alter the results. Therefore, experimental analysis is still necessary to create a baseline for which computer modeling can obtain data. Additionally, simplified, scaled experiments are useful to determine the fundamental relationships between dimensionless terms that drive the physics.

Additional relationships between dimensionless terms will give researchers the freedom to match only the required properties of a body and calculate the actual values of a specific trajectory during post processing. For example, if a scaled MMOI of a model could not be matched due to manufacturing processes, the trajectory determined through experiments may differ from the trajectory of the same model with the desired MMOI. However, using dimensionless scaling relationships, the actual values of properties such as angular velocity and translational velocity could be determined.

\section{Chapter 2 Relevant Literature and Prior Findings}

\subsection{Unstable and Unsteady Aerodynamics}

Unsteady and unstable trajectories are sometimes designed into a UAS. Application of this method is used to aid in the design of a scaled model. Simple shapes were used to reduce the need for complex manufacturing. As a result of unstable aerodynamic motion of the body as it rotates through the air, unsteady properties arise. That is, it is desired to induce an unstable trajectory in order to relate the data to the model in question. Contrary to the flight pattern of a standard aircraft, the body in question moves in a complex motion through all six degrees of freedom; it is common for the model to pass through 360 degrees in any, or all, axes. As the body rotates, extreme angles of attack are reached, causing increased drag or lift. Little research has been developed in the field of unstable aerodynamics. The limited material tends to study common occurrences within nature to develop numerical simulations.

For example, leaves falling from trees or cards falling off a table are phenomena that happen so often, they are often ignored. However, a study by Andersen [6] used these everyday occurrences to observe the unsteady nature of a falling body, induced by instability. That is, under 
certain conditions, the playing card transitioned between fluttering, tumbling, and steady decent. Using numerical simulations derived from the two-dimensional Navier-Stokes equations, a relationship between thickness-to-width ratio and Reynolds number was used to predict the behavior of the playing card (Figure 2.1). For the experiment, $\beta$ was used to represent the thickness to diameter ratio of the disk.
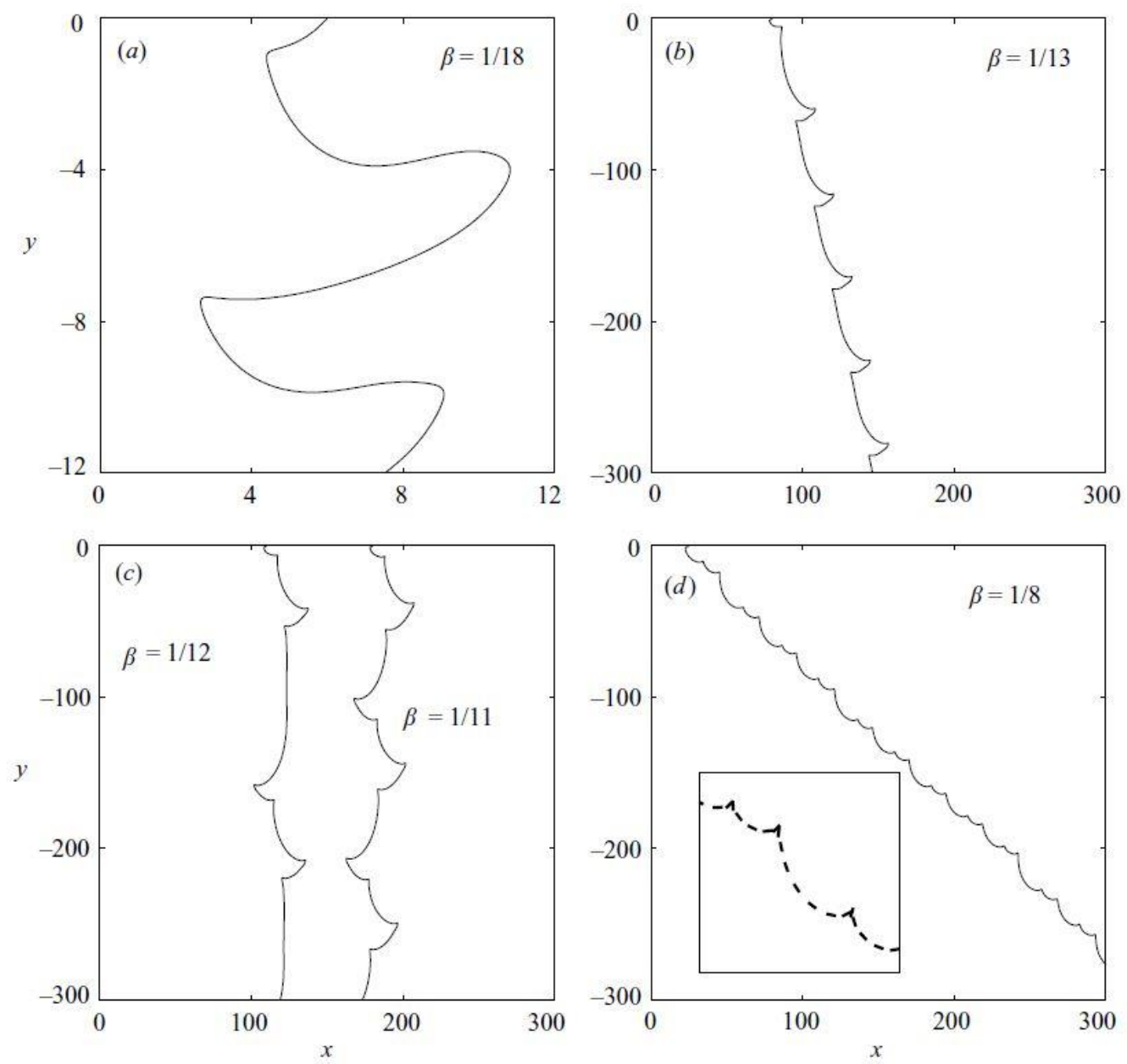

Figure 2.1: Trajectories obtained from direct numerical simulations of the two-dimensional Navier-Stokes equation for freely falling plates in the transition range between fluttering and tumbling: (a) periodic fluttering with the non-dimensional period of oscillation $T=8.1$, (b) fluttering close to the transition between fluttering and tumbling with $T=17.3$, (c) mixture of fluttering and tumbling with $T=34.1$ for $\beta=1 / 12$, and (d) periodic tumbling with $T=12.1[7]$.

Terms to obtain dimensionless MMOI and Reynolds number used to reduce the NavierStokes equations include a length scale, $L$, and velocity scale, $U$. 


$$
L=a, \quad U=\sqrt{\left(\frac{\rho_{s}}{\rho_{f}}-1\right) g b}
$$

Where $a$ the width of the card, $\rho_{s}$ is the density of the card, $\rho_{f}$ is the density of the fluid, $g$ is the acceleration due to gravity, and $b$ is the thickness of the card. The velocity scale used includes a balance between gravity and drag on the card. From these two scales, the dimensionless MMOI, $I^{*}$, and Reynolds number, $R e$, were determined.

$$
I^{*}=\frac{\rho_{s} b}{\rho_{f} a}, \quad R e=\frac{a U}{v}
$$

The additional term, $v$, represents kinematic viscosity of the fluid. This dimensionless MMOI works for a numerical analysis, but it was desired to have a dimensionless MMOI that can be compared to dimensionless angular velocity for a body with changing MMOI and rotation about the z-axis. Tumbling for this experiment occurred about either the $\mathrm{x}$-axis or the $\mathrm{y}$-axis. No analysis was performed with rotation about the z-axis. Furthermore, dimensionless MMOI was changed by adjusting $\beta$ rather than point masses within the body. For a two-dimensional test of the block in a horizontal plane, the balance between gravity and drag was invalid.

Comparing the numerical simulation work of Andersen [6] to another numerical study performed by Jin and $\mathrm{Xu}$ [8] shows the discrepancies in simulations for different methods. Dimensionless MMOI for an elliptical plate is reduced to:

$$
I^{*}=\frac{h\left(l^{2}+h^{2}\right) \rho_{b}}{2 l^{3} \rho_{f}}
$$

and a rectangular plate,

$$
I^{*}=\frac{8 h\left(l^{2}+h^{2}\right) \rho_{b}}{3 \pi l^{3} \rho_{f}}
$$

In the case of Equation 2-1 and Equation 2-2, $h$ is the thickness of the plate, $l$ is the width, and $\rho_{s}$ and $\rho_{f}$ represent the density of the block and the fluid, respectively. The dimensionless MMOI shows a different approach to the same problem. However, changing the equation as well as using a moving mesh approach gives slightly different results. As with the study done by Andersen [6], Jin and Xu attempted to simulate rotation about the $\mathrm{x}$-axis and $\mathrm{y}$-axis rather than the 
z-axis. A simple position plot for a rectangular plate shows the simulated trajectory over time (Figure 2.2).

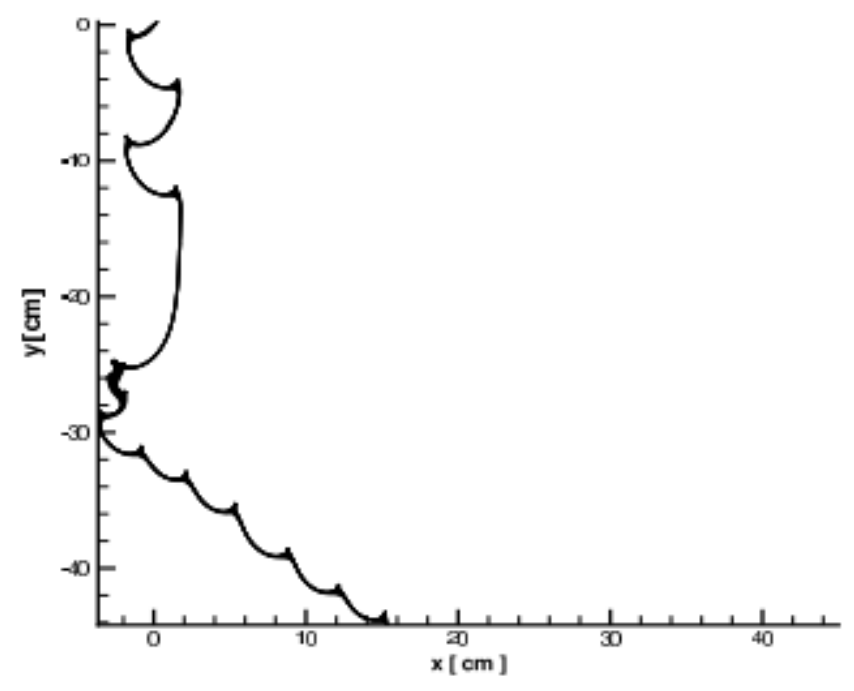

Figure 2.2: Falling trajectory of a rectangle from fluttering to tumbling motion [8].

Some discrepancies observed by Jin and $\mathrm{Xu}$ [8] show that the simulation developed by Andersen [7] produces nearly the same trajectory for elliptical and rectangular plates, under the same conditions. Numerical simulations provide useful estimates of possible body behavior, but the complex fluid mechanics driving the trajectory of each block is difficult to model.

An experimental analysis, such as the one performed by Willmarth [9], with which to compare numerical data further bolsters the understanding of unstable bodies. Studying a variety of disk sizes, Willmarth [9] concluded that falling disks have some dependency on Reynolds number and dimensionless MMOI. Reynolds number associated with stable trajectory was approximately 100 and tumbling did not begin to occur until a Reynolds number greater than 2000 was reached [9].

Snapshots of trailing wakes shown in Figure 2.3 show similar patterns to that of the numerical simulations developed in the previous examples. The snapshots show the wake of the plate for two separate Reynolds number regimes. These wakes can be directly compared to the transition regions of oscillation in Figure 2.1 and Figure 2.2. 

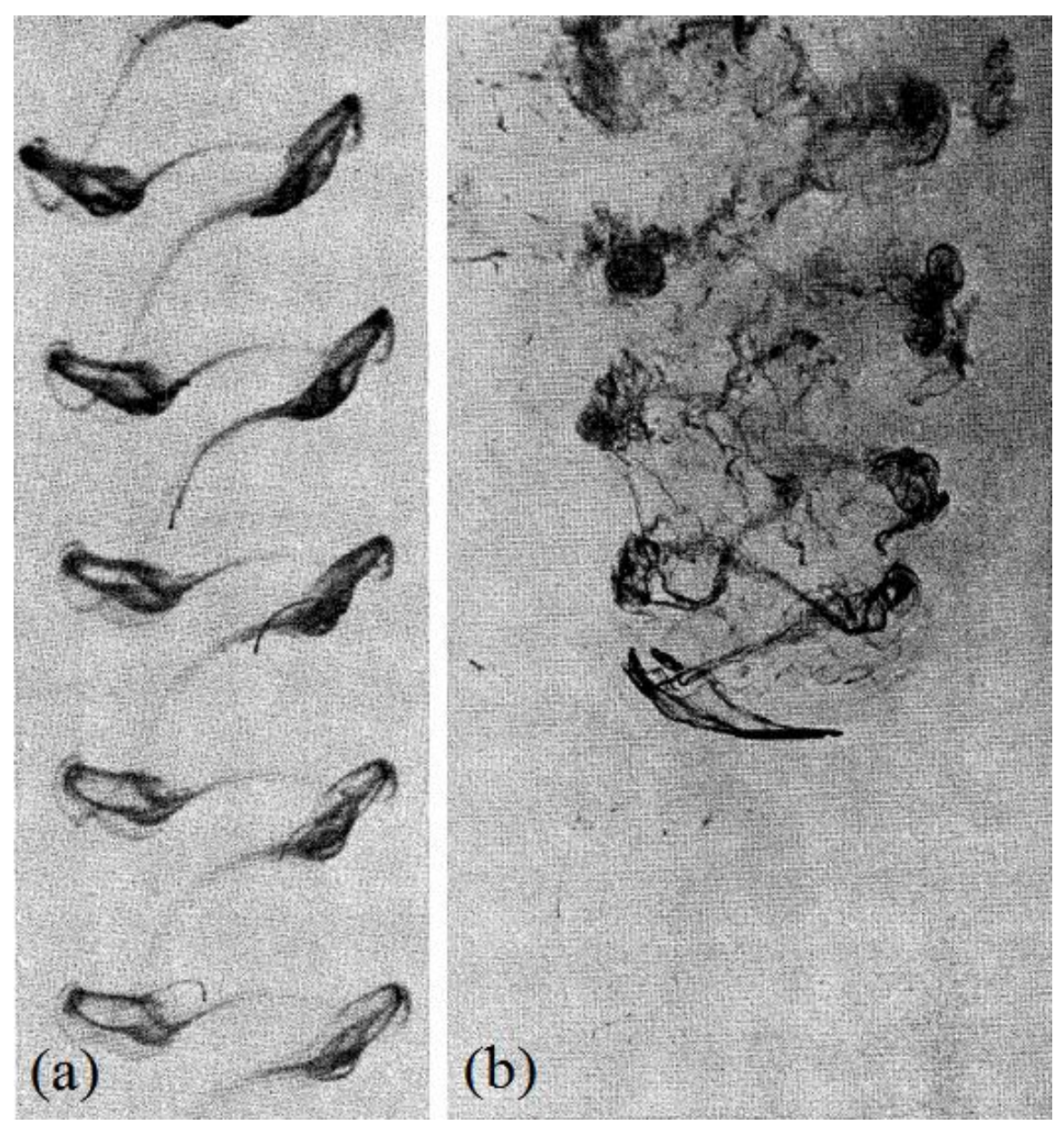

Figure 2.3: (a) View normal to plane of staggered arrangement of two rows of equally spaced $(5 \mathrm{~cm})$ vortex rings left in the wake $40 \mathrm{sec}$ after passage of disk which exhibited oscillatory pitching motion as it fell almost vertically, $R e=170$. (b) Wake of a freely falling disk which exhibited regular translational and pitching oscillations, $R e=$ 1547 [9].

Andersen [6] also conducted another set of experiments for comparison to numerical data. For this experiment, flat plates were dropped in water, rather than air, to lower the kinematic viscosity; consequently, increasing Reynolds number. Using water gives relatively low dimensionless MMOI values as compared with the dimensionless MMOI values of air. From the trajectories shown by Andersen [6] in Figure 2.4, similarities can be seen with those in Figure 2.1. 

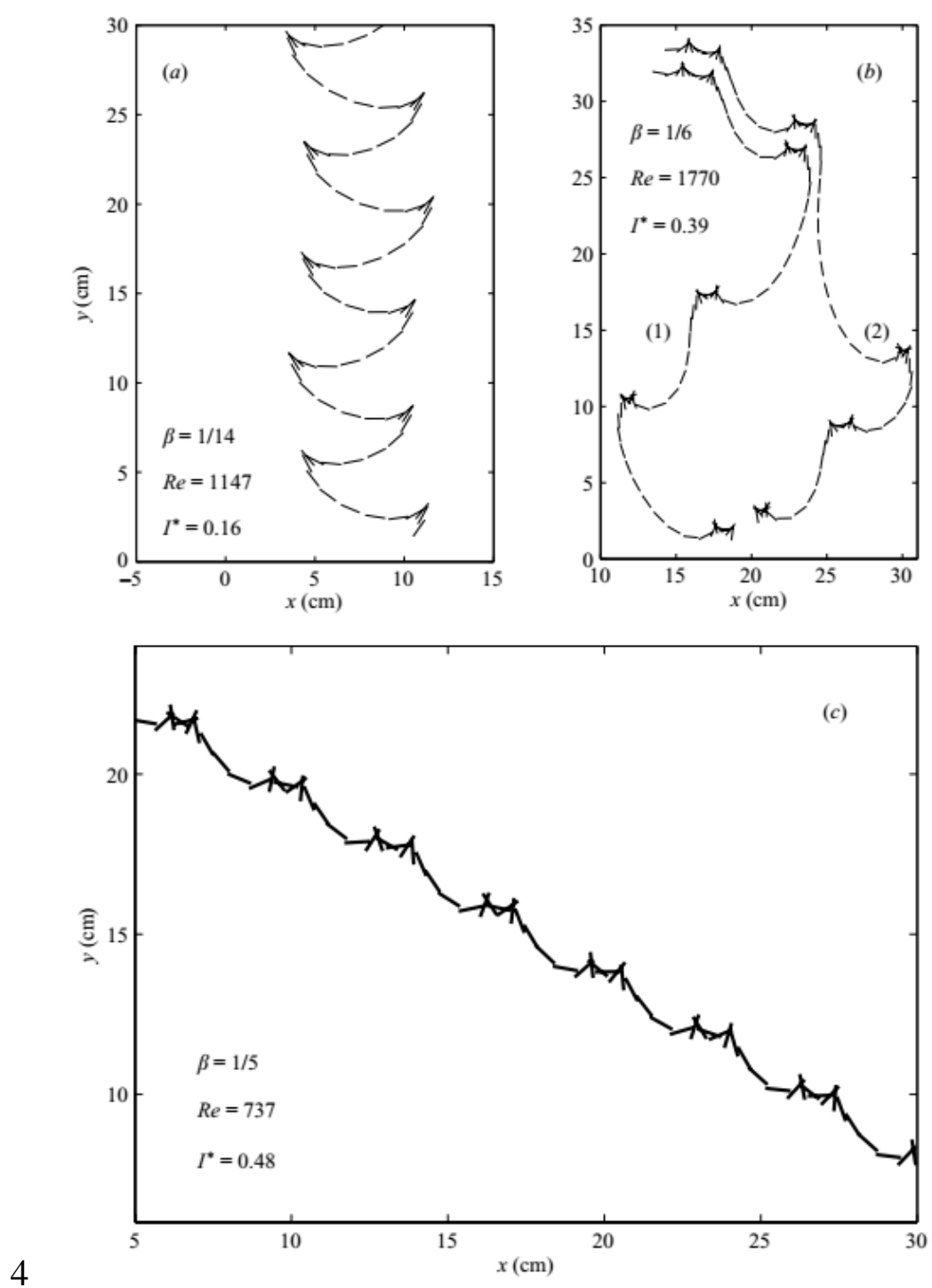

Figure 2.4: Measured plate trajectories: (a) the fluttering plate with $h=0.081 \mathrm{~cm}$ and $\beta=1 / 14$, (b) two apparently chaotic trajectories for the $h=0.162 \mathrm{~cm}$ and $\beta=1 / 6$ plate, and (c) the tumbling plate with $h=0.162 \mathrm{~cm}$ and $\beta=$ $1 / 5$. For clarity the two trajectories in $(b)$ are displaced from each other $0.5 \mathrm{~cm}$ horizontally and $1.5 \mathrm{~cm}$ vertically. The thick line segments show the plate cross-sections with time intervals of $0.04 \mathrm{sec}$ in (a) and $0.025 \mathrm{sec}$ in (b) and (c) $[6]$.

From the experiments, it was found that varying thickness-to-width ratio, dimensionless MMOI, and Reynolds number greatly affect whether the plate tended to flutter or tumble [6]. The experiment also showed signs of purely chaotic behavior for specific cases within certain regimes.

The previously mentioned experiments focused mainly on the relationship between dimensionless mass moment of inertia and Reynolds number for a large number of test articles of varying dimensions or numerical simulations based on the two-dimensional Navier-Stokes equations. Further investigation is needed to determine behavior of rotation about the z-axis for 
different MMOI values. Likewise, limited research has been conducted on changing the MMOI on the same body. It is assumed that for a proper scaling analysis of a model with known MMOI and angular velocity, the dimensionless angular velocity must somehow be related to dimensionless MMOI.

\subsection{Dimensionless Terms}

The experiments covered in the previous section used similar dimensionless terms to organize data into meaningful results. However, these terms change slightly for the experiments on foam blocks, analyzed in this paper. For example, Table 2-1 shows the dimensionless MMOI, $I^{*}$, and length scale used for each experiment covered thus far.

Table 2-1: Accepted dimensionless MMOI terms.

\begin{tabular}{|c|c|}
\hline $\boldsymbol{I}^{*}$ & Source \\
\hline$\frac{\rho_{s} b}{\rho_{f} a}$ & {$[7]$} \\
\hline$\frac{8 h\left(l^{2}+h^{2}\right) \rho_{b}}{3 \pi l^{3} \rho_{f}}$ & {$[8]$} \\
\hline$\frac{I}{\rho_{f} d^{5}}$ & {$[9]$} \\
\hline$\frac{8 h\left(l^{2}+h^{2}\right) \rho_{b}}{3 \pi l^{3} \rho_{f}}$ & {$[6]$} \\
\hline
\end{tabular}

For the purposes of analyzing the blocks in these experiments, the dimensionless MMOI used was that of Willmarth [9], modified to include block density and written as:

$$
I^{*}=\frac{I}{\rho_{b} d^{5}}
$$

and dimensionless angular velocity was calculated as:

$$
\omega^{*}=\frac{\omega_{a v g} d}{u_{x_{a v g}}}
$$

An appropriate length scale must be determined to effectively calculate dimensionless MMOI. The calculated dimensionless MMOI can then be compared to dimensionless angular 
velocity to determine if a scaling factor exists. Different length scales were analyzed to determine a scaling relationship between dimensionless terms. Table 2-2 shows the length scales used for the analysis. Note that Length to Bolt is the only length scale that changes with respect to the block MMOI. Refer to Figure 1.1 for naming convention of block dimensions.

Table 2-2: Length scales used to evaluate scaling relationship between dimensionless terms.

\begin{tabular}{|c|c|c|c|c|c|c|}
\hline $\begin{array}{c}\text { Length } \\
\text { Scale }\end{array}$ & $\begin{array}{c}\text { Aspect } \\
\text { Ratio }\end{array}$ & $\begin{array}{c}\text { Hydraulic } \\
\text { Diameter } \\
{[\mathrm{cm}]}\end{array}$ & $\begin{array}{c}\text { Surface Area } \\
\text { Length } \\
{[\mathrm{cm}]}\end{array}$ & $\begin{array}{c}\text { Length } \\
\text { to Bolt } \\
{[\mathrm{cm}]}\end{array}$ & $\begin{array}{c}\text { Largest } \\
\text { Dimension } \\
{[\mathrm{cm}]}\end{array}$ & $\begin{array}{c}\text { Total Surface Area } \\
{[\mathrm{cm}]}\end{array}$ \\
\hline Equation & $\frac{W}{L}$ & $\frac{4(W L)}{2(W+L)}$ & $\frac{H W}{L}$ & - & $\mathrm{W}$ & $\frac{L W H}{2(L W+H W+L H)}$ \\
\hline
\end{tabular}

\section{Block 1}

\begin{tabular}{|l|l|l|l|l|l|l|}
\hline MMOI 1 & 1.67 & 19.05 & 6.35 & 3.18 & 25.4 & 0.54 \\
\hline MMOI 2 & 1.67 & 19.05 & 6.35 & 6.35 & 25.4 & 0.54 \\
\hline MMOI 3 & 1.67 & 19.05 & 6.35 & 9.53 & 25.4 & 0.54 \\
\hline
\end{tabular}

\section{Block 2}

\begin{tabular}{|l|l|l|l|l|l|l|}
\hline MMOI 1 & 1.67 & 38.10 & 8.47 & 6.35 & 50.8 & 0.79 \\
\hline MMOI 2 & 1.67 & 38.10 & 8.47 & 12.70 & 50.8 & 0.79 \\
\hline MMOI 3 & 1.67 & 38.10 & 8.47 & 19.05 & 50.8 & 0.79
\end{tabular}

\section{Block 3}

\begin{tabular}{|l|l|l|l|l|l|l|}
\hline MMOI 1 & 1.33 & 52.25 & 6.77 & 7.62 & 61.0 & 0.84 \\
\hline MMOI 2 & 1.33 & 52.25 & 6.77 & 15.24 & 61.0 & 0.84 \\
\hline MMOI 3 & 1.33 & 52.25 & 6.77 & 22.86 & 61.0 & 0.84 \\
\hline
\end{tabular}

\section{Block 4}

\begin{tabular}{|l|l|l|l|l|l|l|}
\hline MMOI 1 & 2.51 & 21.73 & 12.73 & 4.8 & 38.1 & 0.68 \\
\hline MMOI 2 & 2.51 & 21.73 & 12.73 & 9.5 & 38.1 & 0.68 \\
\hline MMOI 3 & 2.51 & 21.73 & 12.73 & 14.3 & 38.1 & 0.68 \\
\hline
\end{tabular}

\section{Chapter 3 Block Manufacturing, MMOI Calculations, and Tracking Block}

Prior to testing, an analysis was done to investigate the repeatability and tolerances of the block manufacturing process. Five foam blocks of each size were developed using a hot-wire cutter and cutting jig. A lot of attention was provided to creating accurate dimensions. Therefore, the jig was developed to cut the foam as straight and square as possible. This manufacturing process must be able to be replicated in case of excessive damage to the test specimen. Originally, only three blocks were going to be used for testing. After testing began on the air table, it was 
decided that another block with a relatively higher aspect ratio was needed. Therefore, Block 4 was manufactured at a later time to further investigate scaling relationships and does not appear in the manufacturing analysis.

\subsection{Selecting the Foam}

Kingspan Insulation R 10 Unfaced Polystyrene Foam Board from Lowe's was selected for this study; the foam was selected based on manufacturing capabilities, availability, and price. Foam board used for these experiments comes in 2-in x 4-ft x 8-ft sections, making it the ideal choice for cutting multiple test articles. Another property of the Kingspan Foam Board is that it is closed cell and does not absorb moisture, keeping the density measurement consistent regardless of relative humidity in the room.

\subsection{Cutting the Foam}

Foam blocks were cut using a hot-wire (Figure 3.1) and wooden jig (Figure 3.2). The jig was designed to accommodate blocks up to 24 inches in length and six inches thick. Saws and bits tended to tear out the edges of the foam, creating a rough, uneven surface. Using a hot-wire cutter created a clean cutting edge, unlike a band saw or other cutting method. Running a sharp blade along the foam was another option for creating a clean edge, but the hot-wire cutter proved to be more efficient after initial warm up.

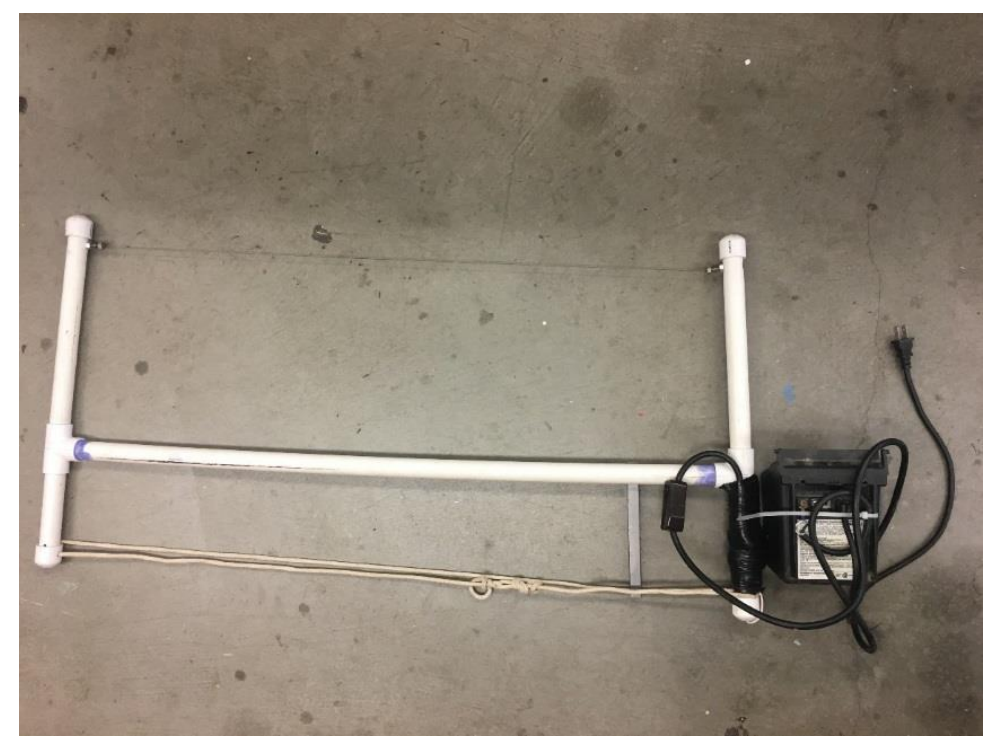

Figure 3.1: Hot-wire used for cutting foam 


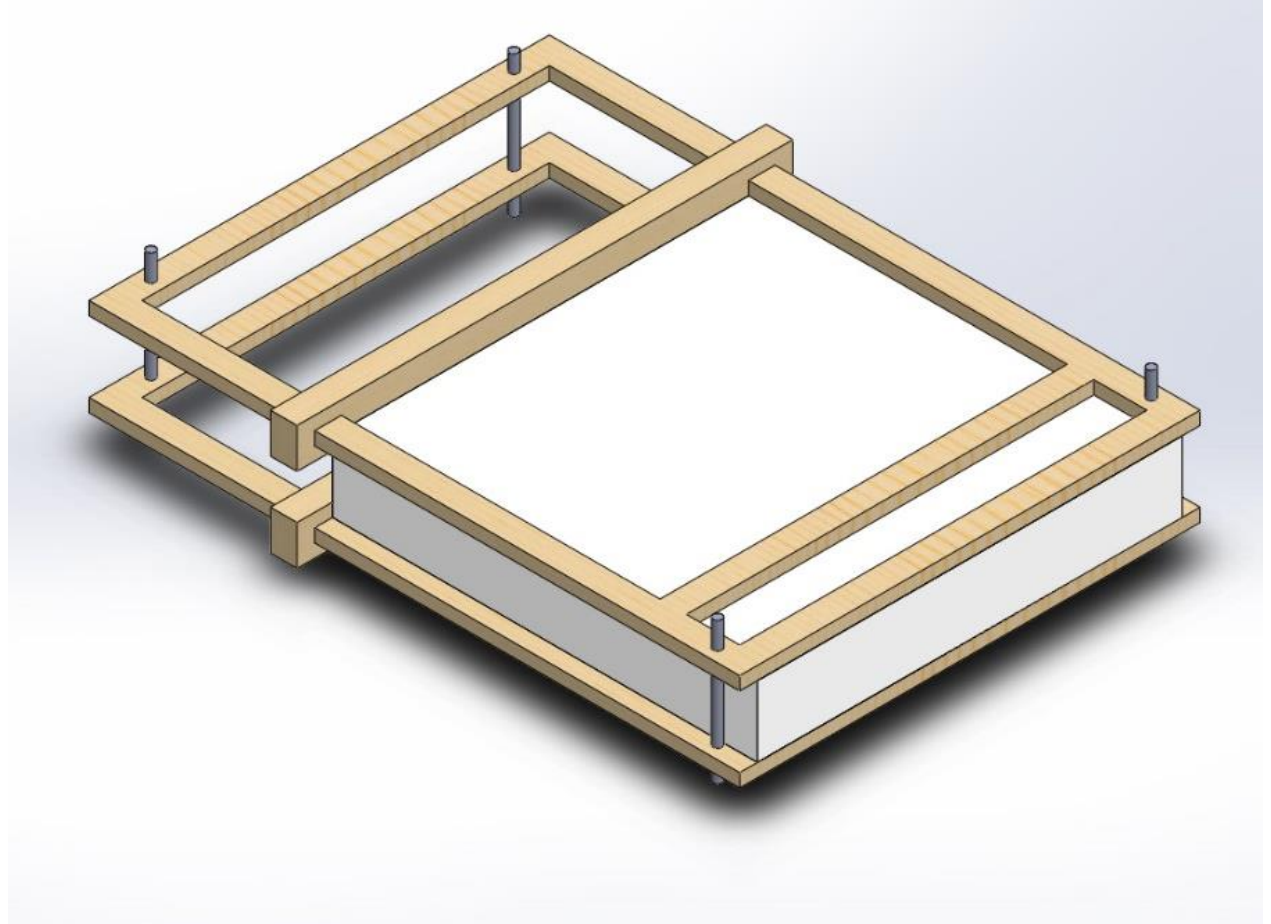

Figure 3.2: CAD model showing wooden jig used to cut foam

The jig worked by placing the sliding stop-block at the rear of the jig to the desired cut length. Then, the rough-cut foam was placed into the jig, while allowing one square side to rest against the stop-block. Since the jig was square, the cut was referenced off of the stop-block, creating two parallel sides on the block at the desired length. This process was then repeated for the adjacent side. Note that for manufacturing of Block 1 , the height, $H$, was reduced from two inches to one and one-half inches. The jig can be designed to accommodate a large range of block sizes by scaling the dimensions up or down, based on desired test article size constraints. The use of a hand held hot-wire cutter created a clean cut quickly and efficiently. An example of a block being adjusted in the jig and cut is shown in Figure 3.3 and Figure 3.4, respectively. The desired dimensions of blocks, as well as the repeatability analysis, is shown in Table 3-1. 


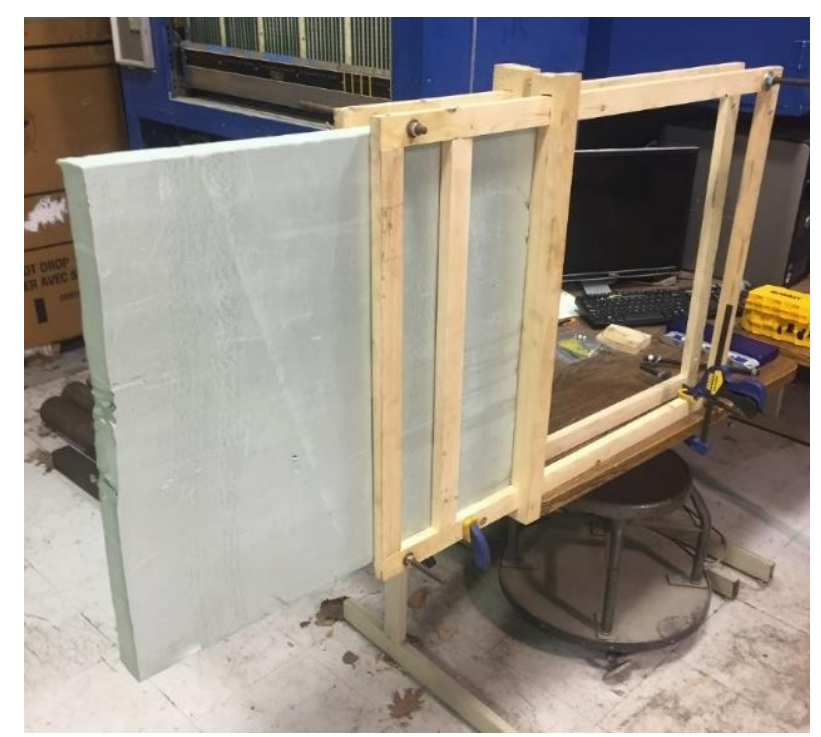

Figure 3.3: Example of foam manufacturing process.

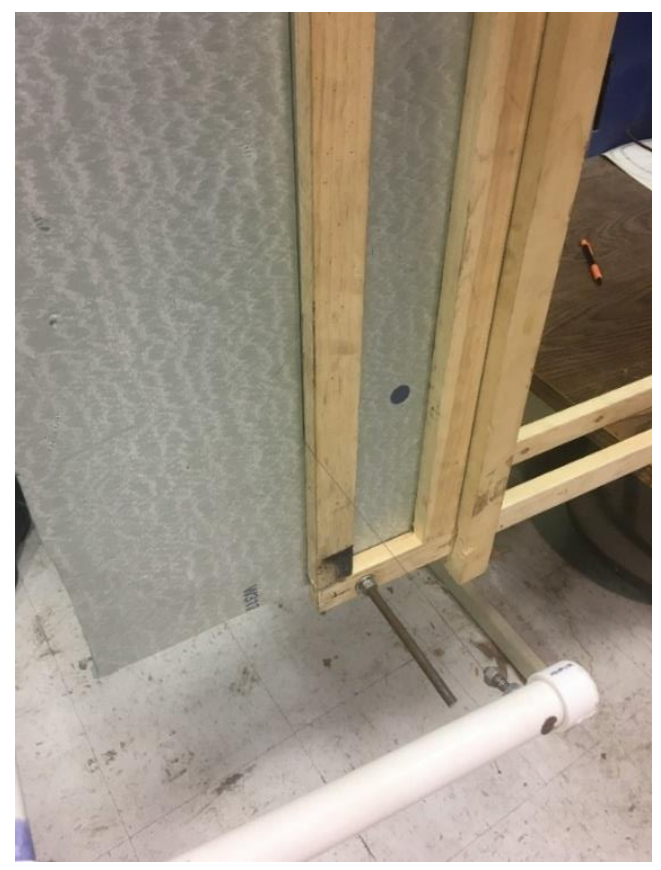

Figure 3.4: Example of hot-wire cutting foam.

To determine repeatability of the foam block manufacturing process, five blocks of each size were created for a total of 15 blocks. However, sample 4 of the Block 1 and sample 2 of the Block 2 were modified to accept an Inertial Measurement Unit (IMU) before a mass was calculated. The IMU was added for the purposes of testing its capabilities to measure position and rotation of the block for a separate study. IMU data is omitted from this study. The height percent difference for Block 2 and Block 3 is not available due to no change to the thicknesses. As previously stated, 
only the height of the Block 1 specimens was modified from two inches to one and one-half inches. Measurements for length, width, and height were taken at each end of the block, and the greatest value from the desired measurement was recorded. For example, when measuring the length of sample 1 of the Block 1, one side measured 5.98 inches while the other side measured 5.97 inches. The value 5.97 inches was recorded, since it is farther from the desired value of 6 inches. The height of each block was measured using a digital micrometer with a resolution of 0.001 inches. The length and width of the blocks were out of the range of the micrometer's measuring capabilities, so dimensions were recorded using a tape measure with a resolution of 0.0625 inches. Measurement techniques for mass and lengths are shown in Figure 3.5 and Figure 3.6, respectively. Percent difference was calculated using the relationship:

$$
\text { Percent Difference }=\left|\frac{\text { Desired Value }- \text { Measured Value }}{\text { Desired Value }}\right| \times 100
$$

Density of the foam material was calculated by first measuring the mass of each block, then dividing the mass by the measured dimensions. An average density was used in calculations for MMOI in CAD. When holes were later added for the additional weights, the density of the foam material remained constant even though the mass changed due to removal of material. Masses of the blocks were measured using an ACCULAB EC-2100 scale, with a resolution of 1 gram. Block masses were measured in ounces and then converted to grams.
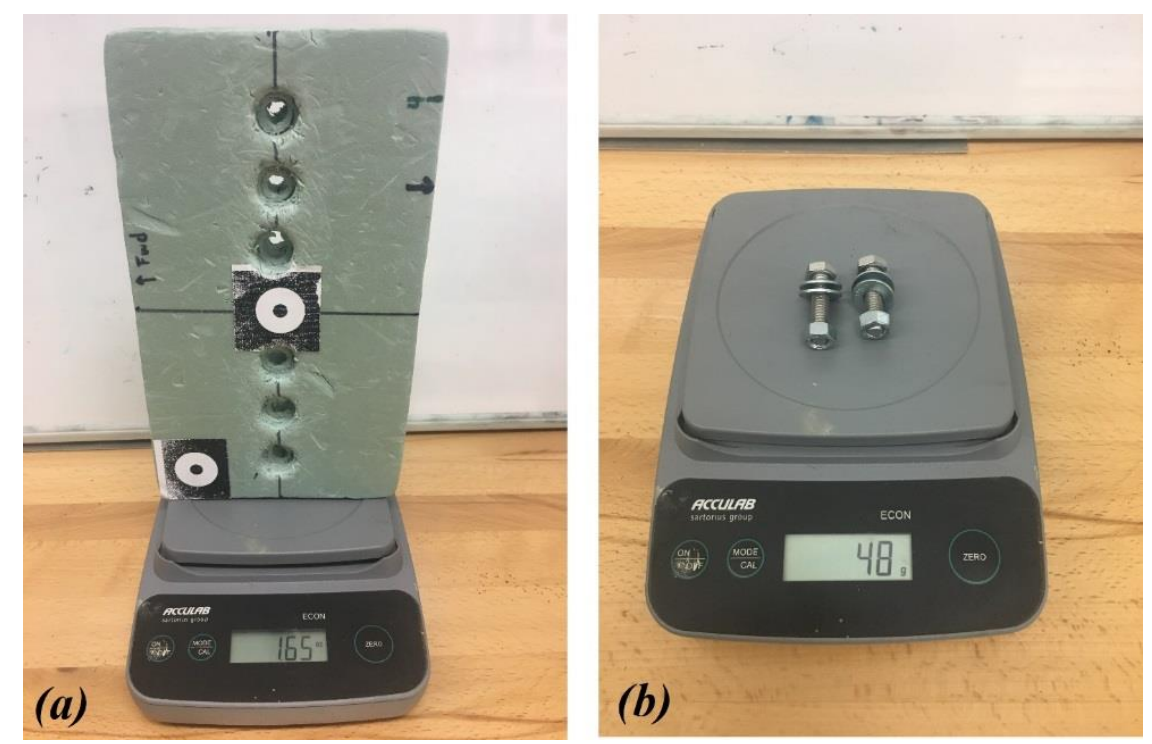

Figure 3.5: Weighing technique for foam blocks and additional masses. Foam measured in ounces and then converted to grams while bolts measured in grams initially. 

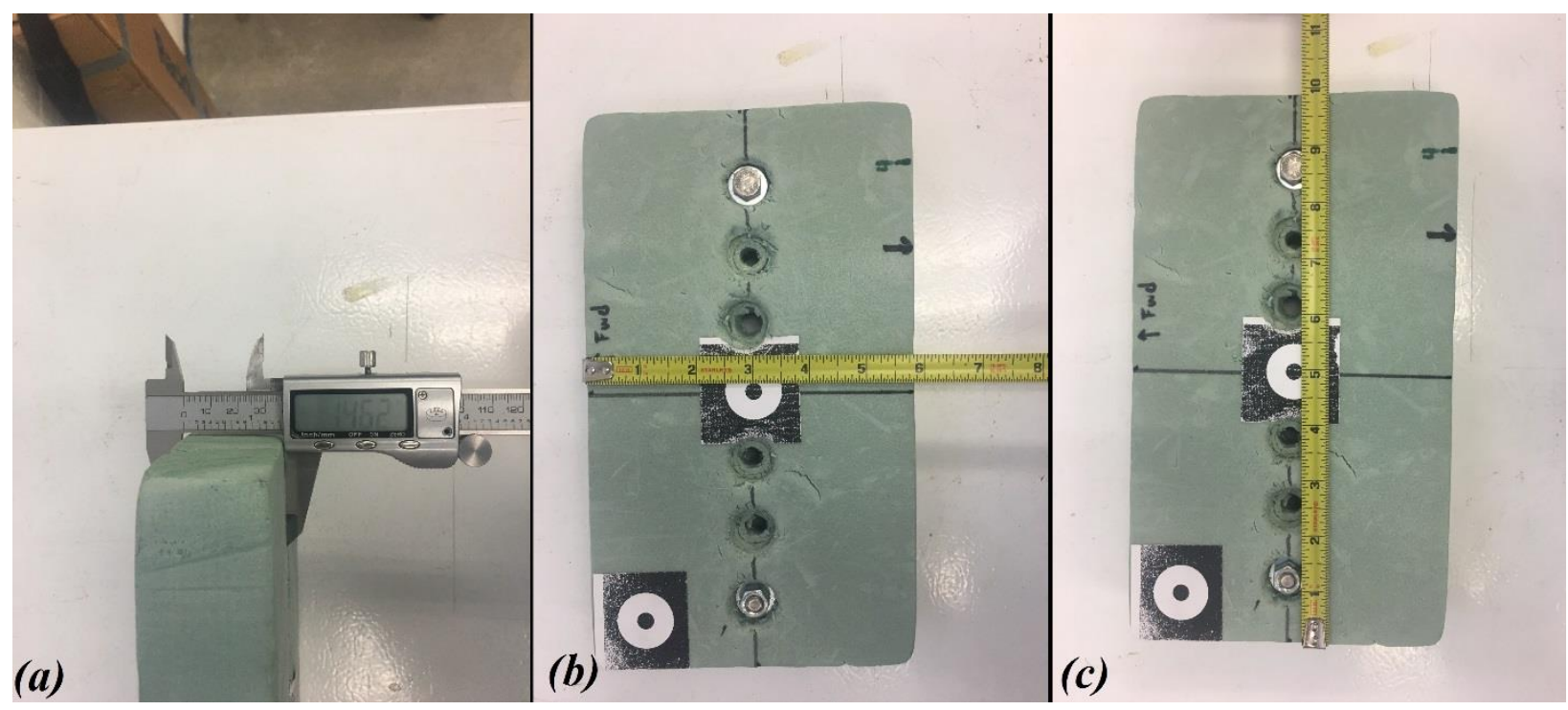

Figure 3.6: Block measurement techniques. (a) Height measurement taken using digital micrometer. (b) Length measurement taken using tape measure. (c) Width measurement taken using tape measure.

Table 3-1: Foam block manufacturing repeatability analysis.

\begin{tabular}{|c|c|c|c|c|c|c|c|c|c|c|c|c|}
\hline & & \multicolumn{3}{|c|}{ Desired Geometry } & \multicolumn{4}{|c|}{ Measured Geometry } & \multicolumn{4}{|c|}{ Calculated Properties } \\
\hline & Sample & Length [in.] & Width [in.] & Height [in.] & Length [in.] & Width [in.] & Height [in.] & Weight [oz] & $\begin{array}{c}\text { Specific Weight } \\
{\left[\mathrm{lbs} / \mathrm{ft}^{\wedge} 3\right]}\end{array}$ & $\begin{array}{l}\text { Length Percent } \\
\text { difference }\end{array}$ & $\begin{array}{l}\text { Width Percent } \\
\text { Difference }\end{array}$ & $\begin{array}{c}\text { Height Percen } \\
\text { Difference }\end{array}$ \\
\hline \multirow{5}{*}{$\begin{array}{l}\vec{r} \\
\text { 음 } \\
\text { D. }\end{array}$} & 1 & 6 & 10 & 1.5 & 5.97 & 9.9375 & 1.53 & 1.7 & 2.02 & $0.50 \%$ & $0.63 \%$ & $2.00 \%$ \\
\hline & 2 & 6 & 10 & 1.5 & 5.96 & 9.90625 & 1.46 & 1.65 & 2.07 & $0.67 \%$ & $0.94 \%$ & $2.67 \%$ \\
\hline & 3 & 6 & 10 & 1.5 & 5.94 & 10 & 1.48 & 1.6 & 1.97 & $1.00 \%$ & $0.00 \%$ & $1.33 \%$ \\
\hline & 4 & 6 & 10 & 1.5 & 5.94 & 9.96875 & 1.49 & $* * *$ & $* * *$ & $1.00 \%$ & $0.31 \%$ & $0.67 \%$ \\
\hline & 5 & 6 & 10 & 1.5 & 5.96 & 10 & 1.49 & 1.6 & 1.95 & $0.67 \%$ & $0.00 \%$ & $0.67 \%$ \\
\hline \multirow{5}{*}{$\begin{array}{l}\stackrel{\sim}{\breve{o}} \\
\text { o }\end{array}$} & 1 & 12 & 20 & 2 & 11.875 & 20 & $\mathrm{n} / \mathrm{a}$ & 8.75 & 1.99 & $1.04 \%$ & $0.00 \%$ & $\mathrm{n} / \mathrm{a}$ \\
\hline & 2 & 12 & 20 & 2 & 11.75 & 19.875 & $\mathrm{n} / \mathrm{a}$ & $* * *$ & $* * *$ & $2.08 \%$ & $0.63 \%$ & $\mathrm{n} / \mathrm{a}$ \\
\hline & 3 & 12 & 20 & 2 & 11.9375 & 19.875 & $\mathrm{n} / \mathrm{a}$ & 8.75 & 1.99 & $0.52 \%$ & $0.63 \%$ & $\mathrm{n} / \mathrm{a}$ \\
\hline & 4 & 12 & 20 & 2 & 11.875 & 19.75 & $n / a$ & 8.65 & 1.99 & $1.04 \%$ & $1.25 \%$ & $n / a$ \\
\hline & 5 & 12 & 20 & 2 & 11.875 & 20.125 & $\mathrm{n} / \mathrm{a}$ & 7.75 & 1.75 & $1.04 \%$ & $0.63 \%$ & $\mathrm{n} / \mathrm{a}$ \\
\hline \multirow{5}{*}{$\begin{array}{l}m \\
\text { ㅁ } \\
\text { 음 }\end{array}$} & 1 & 18 & 24 & 2 & 17.9375 & 23.875 & $\mathrm{n} / \mathrm{a}$ & 13.75 & 1.73 & $0.35 \%$ & $0.52 \%$ & $\mathrm{n} / \mathrm{a}$ \\
\hline & 2 & 18 & 24 & 2 & 18 & 23.75 & $n / a$ & 13.9 & 1.76 & $0.00 \%$ & $1.04 \%$ & $n / a$ \\
\hline & 3 & 18 & 24 & 2 & 18.125 & 24 & $n / a$ & 15.85 & 1.97 & $0.69 \%$ & $0.00 \%$ & $n / a$ \\
\hline & 4 & 18 & 24 & 2 & 18 & 23.9375 & $\mathrm{n} / \mathrm{a}$ & 16 & 2.01 & $0.00 \%$ & $0.26 \%$ & $\mathrm{n} / \mathrm{a}$ \\
\hline & 5 & 18 & 24 & 2 & 18.125 & 24 & $n / a$ & 15.9 & 1.97 & $0.69 \%$ & $0.00 \%$ & $n / a$ \\
\hline
\end{tabular}

It can be seen in Table 3-1 that the largest percent difference of any measurement was $2.67 \%$ and the average percent difference over all the measurements was $0.73 \%$. The percent difference increased for blocks that may have been inserted into the jig at a slight angle or obtained damage from mishandling. However, most cuts were made with a negligible percent difference of less than $2 \%$ from the desired dimensions. Note that the spaces marked with a “***" were removed from the study before their specific weight was measured. These blocks were used in a separate study that will not be detailed within this paper.

Before the blocks were modified to accept additional weights, each block was weighed to calculate the density of the material. This will later be used in Solidworks to numerically calculate the mass moment of inertia of each of the blocks. Solidworks was used to simplify the MMOI calculation due the addition of steel weights to the blocks creating a nonhomogeneous specimen. 
To manufacture all the samples, numerous sheets of foam were cut to size. Featured in Table 3-1 are the slight differences in the foam board density in sample 5 of Block 2 and sample 1 and 2 of Block 3. However, using Chauvenet's Criteria on the data, these points could not be omitted from the average specific weight of the material. Therefore, the average specific weight of the material is $1.94 \mathrm{lb} / \mathrm{ft}^{3}$. This specific weight relates to a density of $0.06 \mathrm{slug} / \mathrm{ft}^{3}$ by Newton's Second Law:

$$
m=\frac{F}{a}
$$

Where $m$ is the mass of the block, $F$ is the force exerted on the scale, and $a$ is acceleration due to gravity, $32.2 \mathrm{ft} / \mathrm{s}^{2}$.

For ease of use during calculations, dimensions were converted from standard to metric and shown in Table 3-2 below. Converting to metric units also provides a better representation of foam density for use in Solidworks.

Table 3-2: Foam block manufacturing repeatability analysis converted to metric units.

\begin{tabular}{|c|c|c|c|c|c|c|c|c|c|c|c|}
\hline & \multicolumn{3}{|c|}{ Desired Geometry } & \multicolumn{4}{|c|}{ Measured Geometry } & \multicolumn{4}{|c|}{ Calculated Properties } \\
\hline Sample & Length $[\mathrm{cm}]$ & Width $[\mathrm{cm}]$ & Height $[\mathrm{cm}]$ & Length $[\mathrm{cm}]$ & Width $[\mathrm{cm}]$ & Height $[\mathrm{cm}]$ & Mass [g] & $\begin{array}{c}\begin{array}{c}\text { Density } \\
{\left[\mathrm{g} / \mathrm{cm}^{\wedge} 3\right]}\end{array} \\
\end{array}$ & $\begin{array}{l}\text { Length Percent } \\
\text { difference }\end{array}$ & $\begin{array}{c}\text { Width Percent } \\
\text { Difference }\end{array}$ & $\begin{array}{l}\text { Height Percent } \\
\text { Difference }\end{array}$ \\
\hline 1 & 15.240 & 25.400 & 3.810 & 15.164 & 25.241 & 3.886 & 48.2 & 0.032 & $0.50 \%$ & $0.62 \%$ & $2.00 \%$ \\
\hline 2 & 15.240 & 25.400 & 3.810 & 15.138 & 25.162 & 3.708 & 46.8 & 0.033 & $0.67 \%$ & $0.94 \%$ & $2.67 \%$ \\
\hline 3 & 15.240 & 25.400 & 3.810 & 15.088 & 25.400 & 3.759 & 45.4 & 0.032 & $1.00 \%$ & $0.00 \%$ & $1.33 \%$ \\
\hline 4 & 15.240 & 25.400 & 3.810 & 15.088 & 25.321 & 3.785 & $* * *$ & $n / a$ & $1.00 \%$ & $0.31 \%$ & $0.67 \%$ \\
\hline 5 & 15.240 & 25.400 & 3.810 & 15.138 & 25.400 & 3.785 & 45.4 & 0.031 & $0.67 \%$ & $0.00 \%$ & $0.67 \%$ \\
\hline 1 & 30.480 & 50.800 & 5.080 & 30.163 & 50.800 & $n / a$ & 248.1 & 0.032 & $1.04 \%$ & $0.00 \%$ & $\mathrm{n} / \mathrm{a}$ \\
\hline 2 & 30.480 & 50.800 & 5.080 & 29.845 & 50.483 & $\mathrm{n} / \mathrm{a}$ & $* * *$ & $\mathrm{n} / \mathrm{a}$ & $2.08 \%$ & $0.62 \%$ & $\mathrm{n} / \mathrm{a}$ \\
\hline 3 & 30.480 & 50.800 & 5.080 & 30.321 & 50.483 & $\mathrm{n} / \mathrm{a}$ & 248.1 & 0.032 & $0.52 \%$ & $0.62 \%$ & $\mathrm{n} / \mathrm{a}$ \\
\hline 4 & 30.480 & 50.800 & 5.080 & 30.163 & 50.165 & $n / a$ & 245.2 & 0.032 & $1.04 \%$ & $1.25 \%$ & $\mathrm{n} / \mathrm{a}$ \\
\hline 5 & 30.480 & 50.800 & 5.080 & 30.163 & 51.118 & $n / a$ & 219.7 & 0.028 & $1.04 \%$ & $0.63 \%$ & $n / a$ \\
\hline 1 & 45.720 & 60.960 & 5.080 & 45.561 & 60.643 & $\mathrm{n} / \mathrm{a}$ & 389.8 & 0.028 & $0.35 \%$ & $0.52 \%$ & $\mathrm{n} / \mathrm{a}$ \\
\hline 2 & 45.720 & 60.960 & 5.080 & 45.720 & 60.325 & $\mathrm{n} / \mathrm{a}$ & 394.1 & 0.028 & $0.00 \%$ & $1.04 \%$ & $\mathrm{n} / \mathrm{a}$ \\
\hline 3 & 45.720 & 60.960 & 5.080 & 46.038 & 60.960 & $n / a$ & 449.3 & 0.032 & $0.69 \%$ & $0.00 \%$ & $\mathrm{n} / \mathrm{a}$ \\
\hline 4 & 45.720 & 60.960 & 5.080 & 45.720 & 60.801 & $\mathrm{n} / \mathrm{a}$ & 453.6 & 0.032 & $0.00 \%$ & $0.26 \%$ & $\mathrm{n} / \mathrm{a}$ \\
\hline \multirow[t]{2}{*}{5} & 45.720 & 60.960 & 5.080 & 46.038 & 60.960 & $\mathrm{n} / \mathrm{a}$ & 450.8 & 0.032 & $0.69 \%$ & $0.00 \%$ & $\mathrm{n} / \mathrm{a}$ \\
\hline & & & & & & & avg & 0.031 & \multicolumn{3}{|c|}{$0.73 \%$} \\
\hline
\end{tabular}

\subsection{Addition of point masses}

To effectively change the MMOI of each bock, bolts, nuts, and washers were added to the foam to represent two additional weights. The mass of one additional weight is half the total weight of the block. For example, Block 1, having an average mass of 46 grams, received an additional 46 grams of weight in the form of two 23 gram bolt, nut, and washer combinations. However, obtaining high accuracy was not feasible without milling customized weights. For this reason, the weights were given a tolerance of $5 \%$ error, shown in Figure 3.8. 
Additionally, adding masses of a different material created nonhomogeneous blocks. That is, the density of the foam blocks was no longer the same with additional masses. To calculate an effective density, the total mass of each block was divided by its total volume, assuming the holes were filled with the additional masses, as shown in Equation 3-1. The change in density was not needed for the Solidworks numerical calculation of MMOI because each bolt, nut, and washer used was modeled separately and assigned a measured mass. Thus, each component remained as a separate part with its own properties.

$$
\rho_{\text {eff }}=\frac{m_{\text {total }}}{\text { Volume }_{\text {total }}}=\frac{m_{\text {block }}+m_{\text {additional mass }}}{\text { Volume }_{\text {block }}} \quad \text { Equation 3-1 }
$$

Special attention was given to balancing the weights along each axis as closely as possible. Weight variance in the $x-y$ axis would have had greater effects on the results of the drop tests. With the goal of effectively changing the MMOI and maintaining a light weight block, it was decided that doubling the block's mass would best meet these conditions. The weights must have enough influence to affect the block's trajectory under the desired initial conditions, as well as keep the blocks light enough to glide across the air table with no contact between the surfaces during the horizontal table test. The maximum allowable mass for the blocks and weights before contact with the air table surface can be seen in

Table 3-3. At the desired $60-65 \%$ output on the voltage regulator, weights were added to each block and tested on the table until a rubbing noise was heard.

Table 3-3: Maximum allowable mass for each block before interference with table surface.

\begin{tabular}{|c|c|c|}
\hline Block Size & $\begin{array}{c}\text { Additional Mass when Contact with } \\
\text { Table Occurs [g] }\end{array}$ & \% of Block Mass \\
\hline Block 1 & 106 & 225 \\
\hline Block 2 & 453 & 189 \\
\hline Block 3 & 1893 & 442 \\
\hline Block 4 & n/a & n/a \\
\hline
\end{tabular}

Additional weights were selected by trial and error. In order to be recessed within the block, the total length of the bolt was required to be shorter than the height of the block. Recessing the additional weights into the block ensured a smoother surface and less change to the aerodynamic geometry of the test article. With the mass requirement in mind, each bolt was selected and weighed. Once a bolt with a mass slightly below the desired value was found, a nut was added to secure it to the foam block. If the mass was still outside the $5 \%$ tolerance, washers 
were added. The final configuration for additional weights for each block size can be seen in Figure 3.7. The holes made in the foam were designed to compress the foam between the larger surfaces of the bolt head and nut or washer, while the bolt shaft is able to go all the way through the foam. The holes closest to the center of the block were designated MMOI 1, the second set of holes MMOI 2, and holes furthest from the center are MMOI 3.

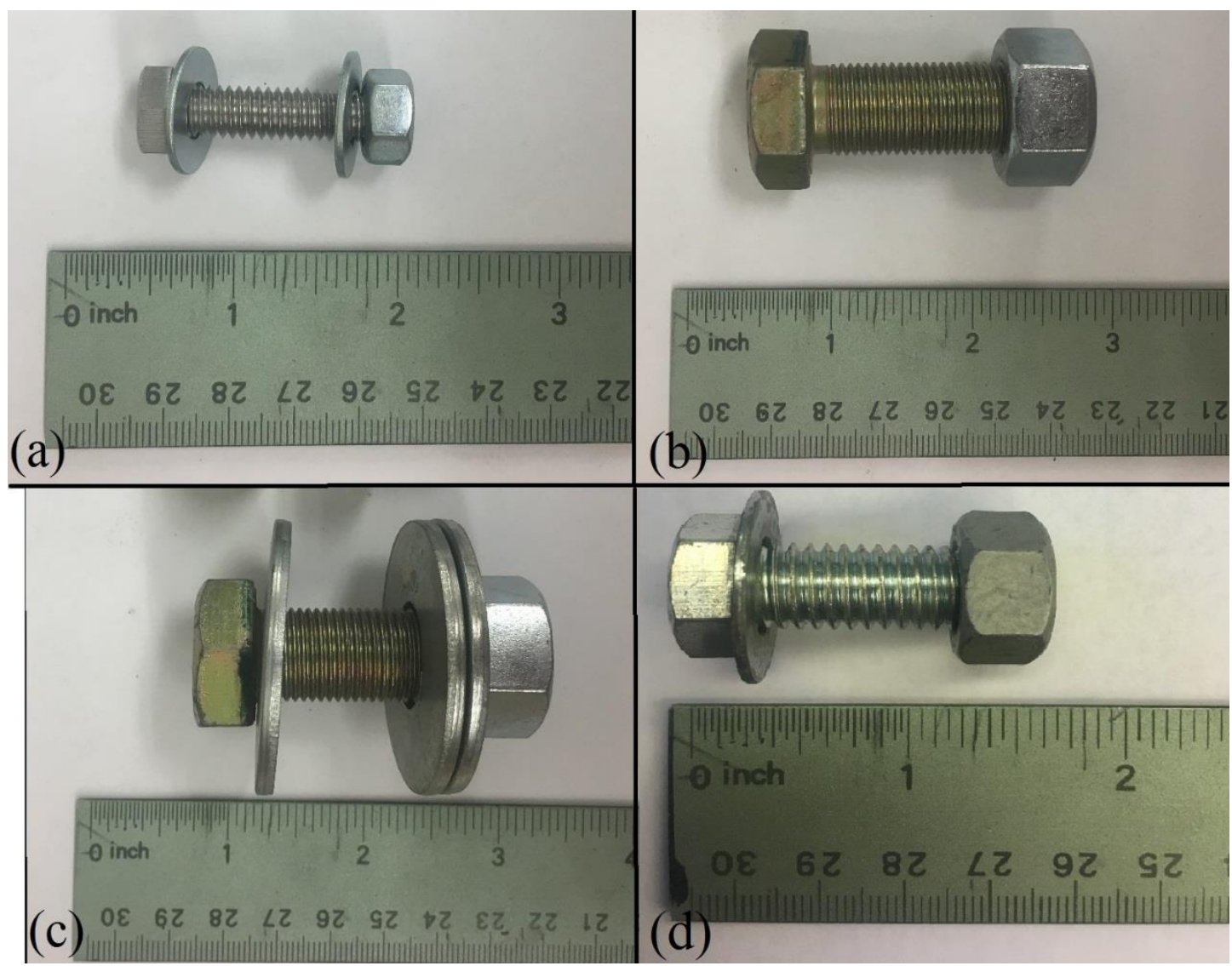

Figure 3.7: Additional weights for changing MMOI of block. (a) Additional weights designed for Block 1. (b) Additional weights designed for Block 2. (c) Additional weights designed for Block 3. (d) Additional weights designed for Block 4. 


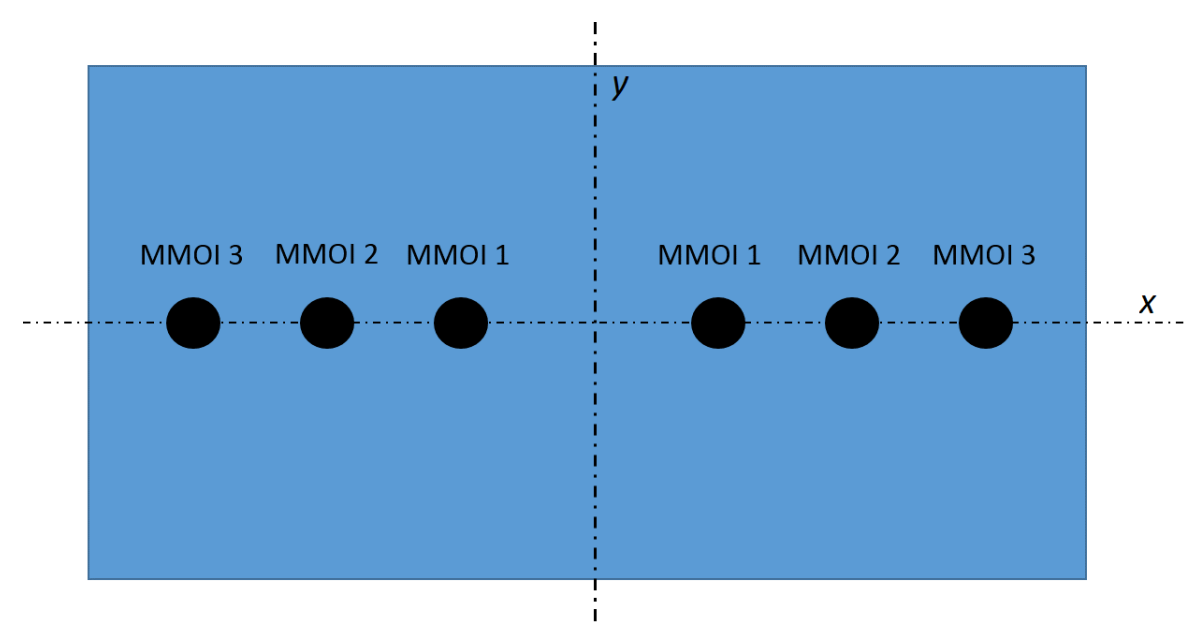

Figure 3.8: MMOI naming convention.

Table 3-4: Additional weight to change MMOI relationship to block mass.

\begin{tabular}{|c|c|c|c|c|c|c|}
\hline $\begin{array}{c}\text { Test } \\
\text { Article }\end{array}$ & $\begin{array}{c}\text { Avg. } \\
\text { Block } \\
\text { Mass } \\
{[\mathbf{g}]}\end{array}$ & $\begin{array}{c}\text { Desired } \\
\text { Single Bolt } \\
\text { Mass } \\
{[\mathbf{g}]}\end{array}$ & $\begin{array}{c}\text { Measured } \\
\text { Bolt 1 Mass } \\
\text { [g] }\end{array}$ & $\begin{array}{c}\text { Measured } \\
\text { Bolt 2 Mass } \\
\text { [g] }\end{array}$ & $\begin{array}{c}\text { Total Mass of } \\
\text { Test Article [g] }\end{array}$ & $\begin{array}{c}\text { Max \% } \\
\text { Error } \\
<\mathbf{5}\end{array}$ \\
\hline Block 1 & 47 & 23.5 & 23.7 & 23.8 & 95 & 1.3 \\
\hline Block 2 & 240 & 120.0 & 117.1 & 117.3 & 473 & 2.4 \\
\hline Block 3 & 428 & 214.0 & 210.8 & 209.3 & 848 & 2.2 \\
\hline Block 4 & 78 & 39.0 & 40.8 & 40.7 & 162 & 4.6 \\
\hline
\end{tabular}

The addition of holes in the blocks affected their aerodynamic properties. To compensate for these voids in the surface, tape was placed over the bolt holes to allow for a uniform surface across the block (Figure 3.9). The tape helped to keep the block surface uniform so the force from the jets could be distributed evenly. 


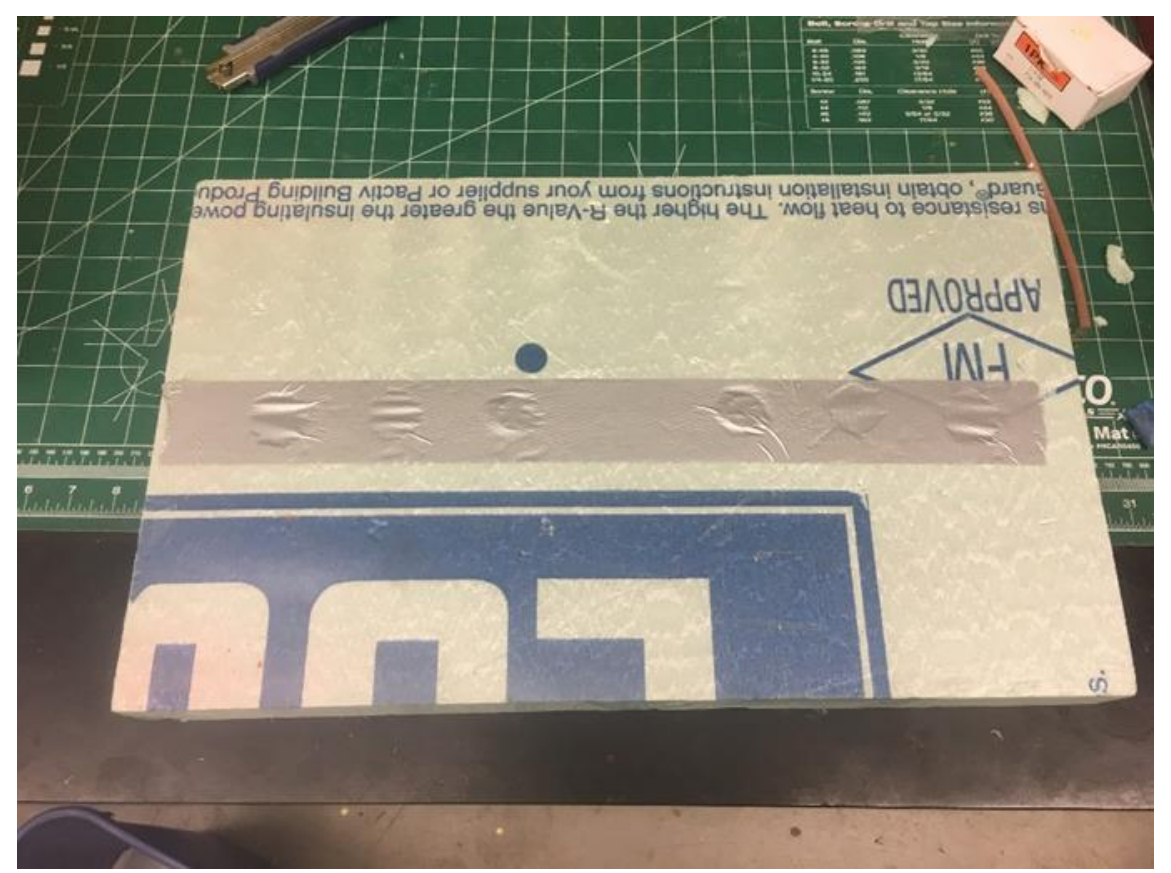

Figure 3.9: Covering holes on blocks to create even surface plane.

\subsection{MMOI Calculation}

For a three-dimensional, homogeneous material without additional point masses, mass moment of inertia about the z-axis can be calculated by:

$$
I_{z z}=\frac{1}{12} m\left(w^{2}+h^{2}\right)
$$

Where $m$ is the mass of the block, $w$ is the width of the block, and $h$ is the height (Figure 1.1). However, the additional weights added to the foam created a non-homogenous material, deeming this equation inaccurate for this situation.

To accurately calculate MMOI, $\mathrm{I}_{\mathrm{zz}}$, about the z-axis of each block size, numerical methods were implemented using Solidworks. A custom material was added to Solidworks to better represent the properties of the foam. The material "Foam" was added to the Solidworks library using the density calculated from Table 3-2 and a Poisson's Ratio of zero. A Poisson's Ratio of zero is merely a simplified assumption for determining MMOI. Therefore, it was not an accurate comparison to the actual foam properties. However, since the foam was not being deformed in tension or compression, other than a small centripetal force, the assumption of zero for Poisson's Ratio was a reasonable simplification. The block was also assumed to be a rigid body. For each 3D block, three MMOIs were calculated. However, only MMOI about the z-axis was analyzed in 
this study. Each bolt, washer, and nut were also represented in Solidworks, and an assembly was created using the same configuration shown in Figure 3.10. Given in units of $\mathrm{g}-\mathrm{cm}^{2}$, a screenshot of the assembly window in Solidworks for the first MMOI of Block 1 is shown as Figure 3.10, and an enhanced view of the mass properties tab is shown as Figure 3.11. The other blocks were analyzed using the same method. Four block sizes and three MMOI possibilities give a total of 12 $I_{z z}$ values. As previously stated, the value for $I_{z z}$ was the only value used for calculations.

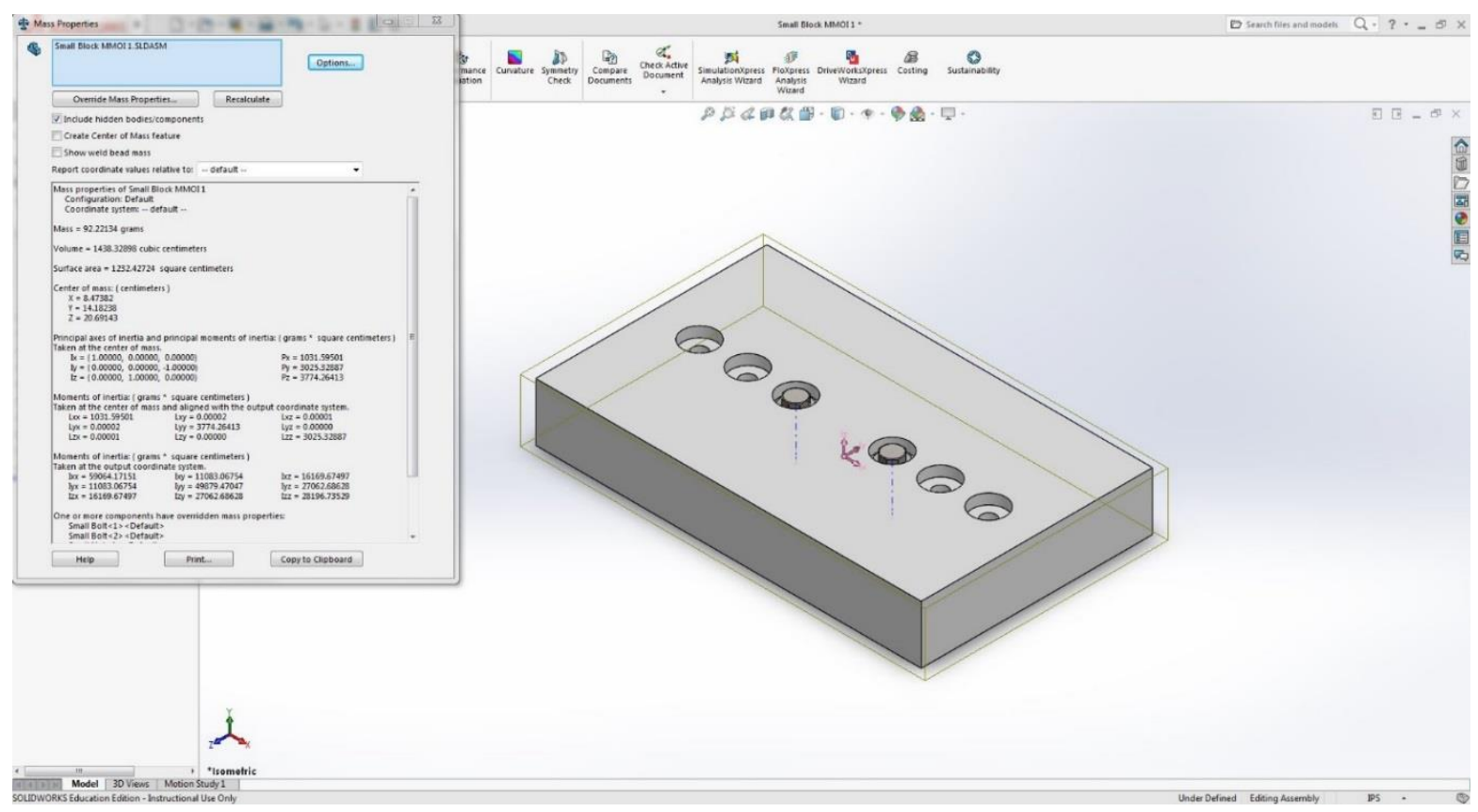

Figure 3.10: Solidworks assembly of Block 1 - MMOI 1 with mass properties window showing calculations. 


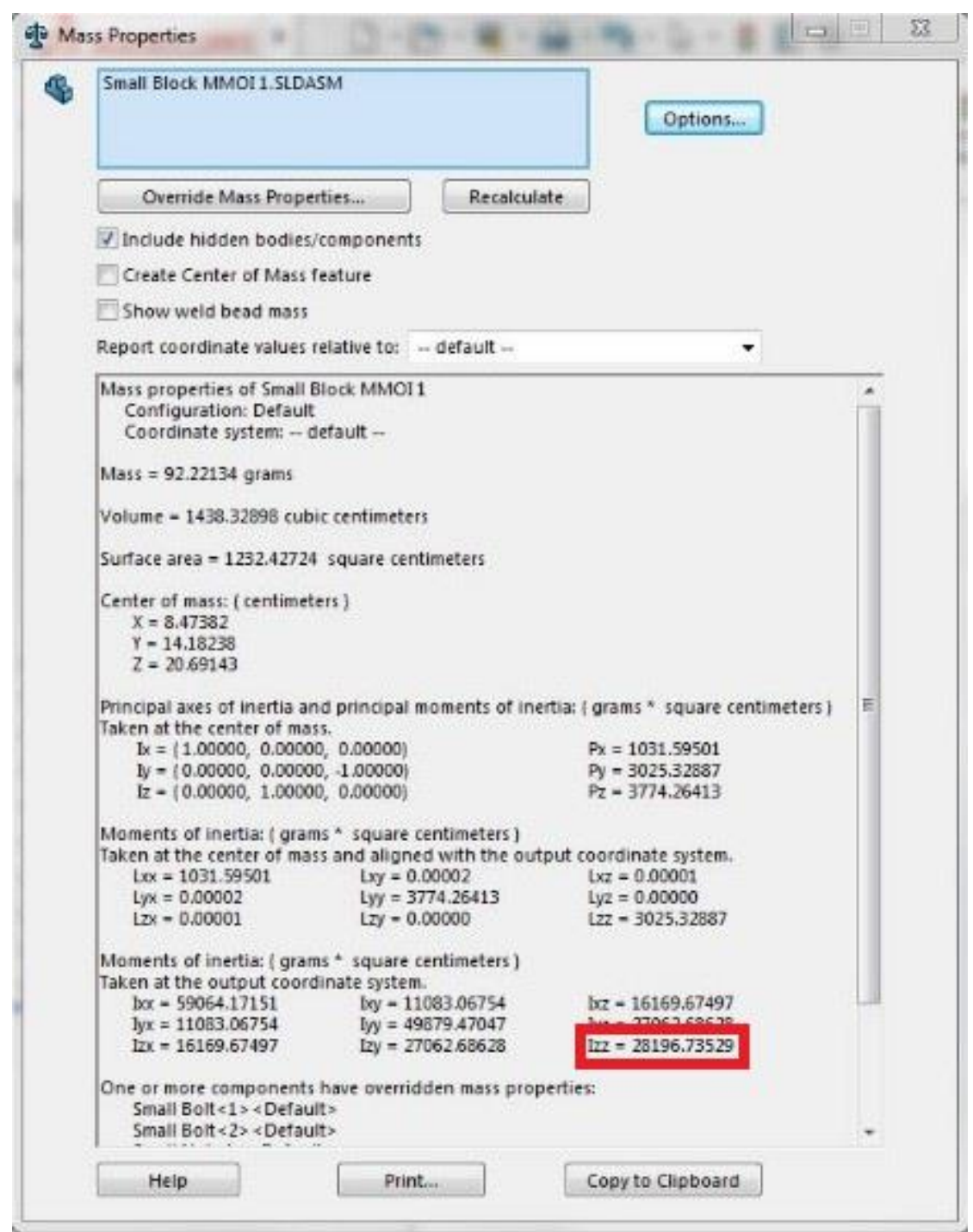

Figure 3.11: Enhanced view of mass properties window and MMOI calculation, shown in red box, for specific configuration of block and additional weights.

Each configuration of block size and additional mass location was constructed in Solidworks and analyzed using the mass properties feature (Table 3-5).

Table 3-5: Numerical MMOI values calculated using Solidworks.

\begin{tabular}{|c|c|c|c|c|}
\hline \multirow{2}{*}{} & Block 1 & Block 2 & Block 3 & Block 4 \\
\cline { 2 - 5 } & $I_{z z}\left(\mathrm{~g}-\mathrm{cm}^{2}\right)$ & $I_{z z}\left(\mathrm{~g}-\mathrm{cm}^{2}\right)$ & $I_{z z}\left(\mathrm{~g}-\mathrm{cm}^{2}\right)$ & $I_{z z}\left(\mathrm{~g}-\mathrm{cm}^{2}\right)$ \\
\hline MMOI 1 & 28196.7 & 638661.3 & 11247770.9 & 117487.4 \\
\hline MMOI 2 & 29642.3 & 667193.6 & 1202314 & 123187.4 \\
\hline MMOI 3 & 32051.6 & 714747.6 & 1331552.5 & 132694.5 \\
\hline
\end{tabular}




\subsection{Modifications for tracking}

The two software programs used to analyze the block trajectories operate using similar principles. However, the complexity of detail greatly differs between them. Each software uses points of interest and a known length scale to determine position of the points as the move through space and time, but the methods used to determine those points is where the Vicon system surpasses the open source tracker software.

The software, Tracker, uses differences in color scale to track points in a video and was employed in the current research effort. Simply, an initial point, within a window of space, was defined in the software as a reference. Tracker then attempts to find that window throughout each frame of the video. The center point within the window was the data point taken for analysis. Chapter 4 explains this process in greater detail. To create the most contrast for Tracker to determine points, black and white stickers were applied to the center of the block and the top left corner as shown in Figure 3.12. The point at the center of the block was tracked with respect to a user defined coordinate system to determine translational velocity, and the point at the corner was tracked with respect to the center point to determine rotational velocity.

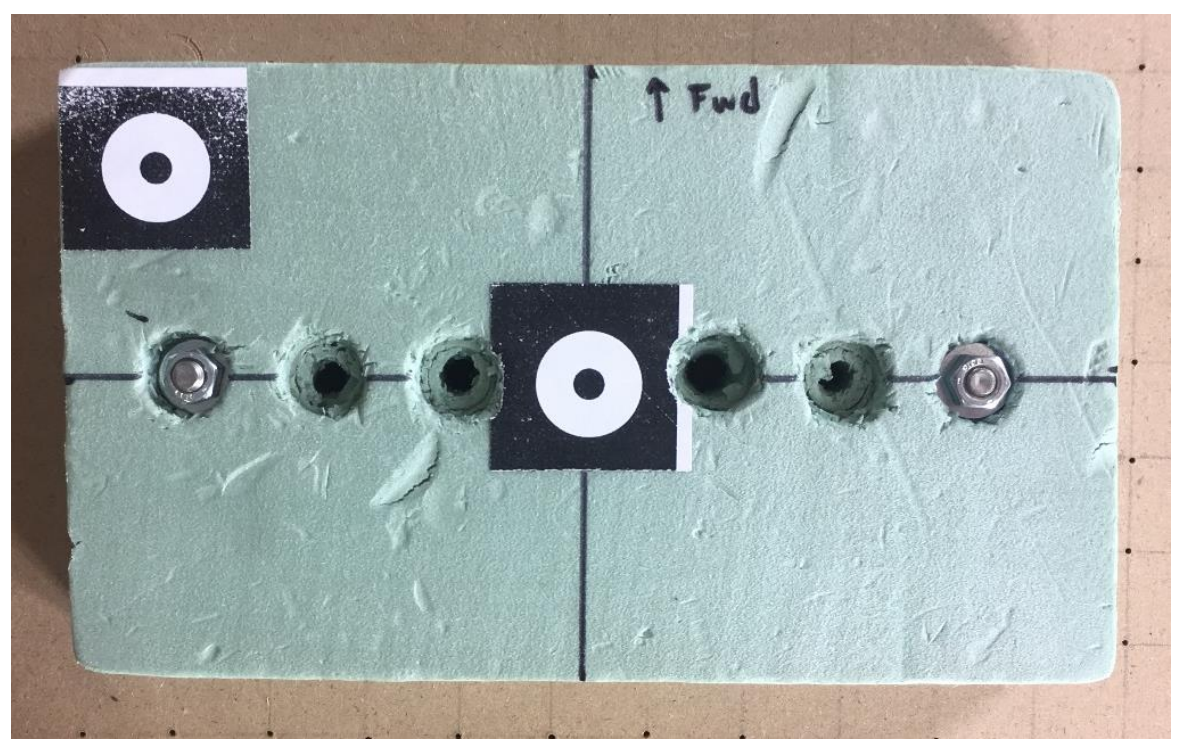

Figure 3.12: Location of high contrast stickers for tracking. The center sticker was used to track translational velocity with respect to a user defined coordinate system while the sticker in the upper left corner was used to track rotational velocity with respect to the center sticker.

The VICON camera system tracks points using infrared light to highlight points of interest. Similar to the software Tracker, VICON cameras "look" for a predetermined shape and white balance that match a specified intensity. Simply, highly reflective flat or spherical markers were 
attached to the test article so the software could generate an object. Using a simple camera flash, the reflective property of the spheres is shown in Figure 3.13. The IR light emitted from the cameras bounces off the reflective markers and back into the lens of the camera. VICON software is then able to determine properties of the object based on marker orientation in relation to the cameras. The software uses the points to compare to the geometry of the accepted object. Figure 3.14 demonstrates an orientation of markers used to create the object. Once an object is created, the markers must remain in the same respective location. Additionally, the orientation of marker placement is very import when using the Vicon system. Markers must be placed so that the object has no symmetry. That is, all markers cannot be in the same plane or be similar distances apart. Creating an unsymmetrical marker orientation allows the system to determine orientation of the object as it rotates through different planes. If markers were placed in a symmetrical pattern, the software would not be able to differentiate whether the block was in one orientation verses another. Each point would be tracked, and inaccurate data would be recorded.

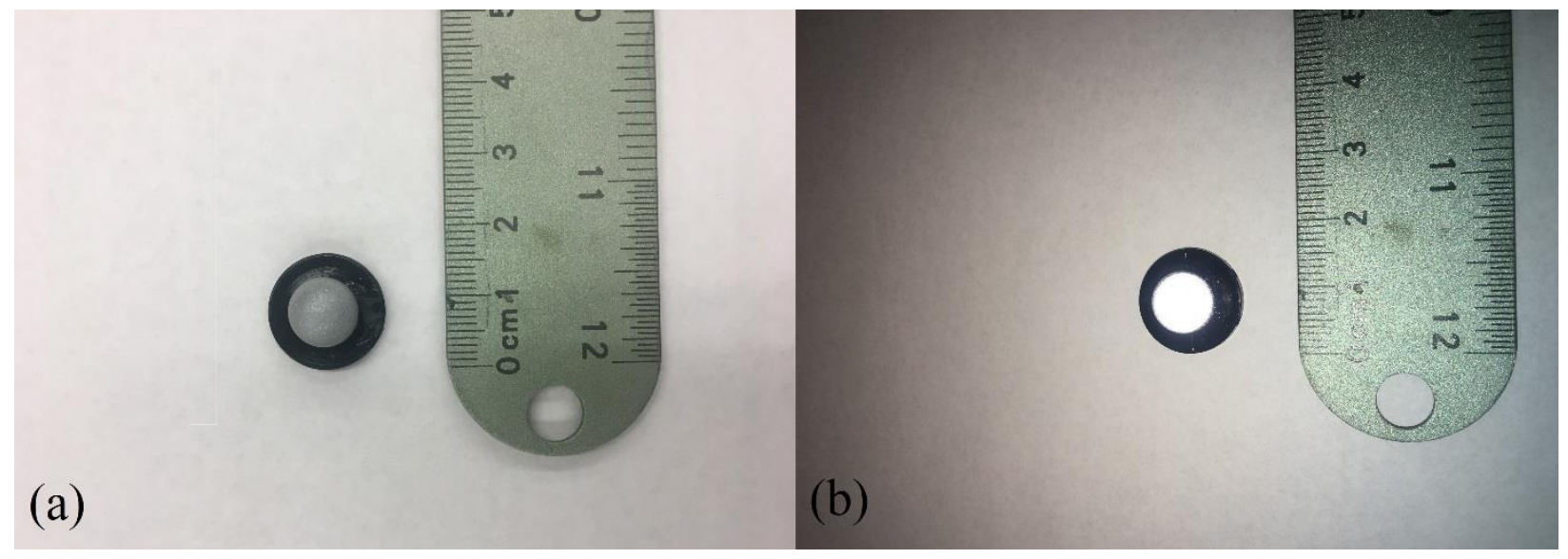

Figure 3.13: Demonstration of reflective property of markers for analysis using Vicon camera system. (a) Picture taken using no flash. (b) Picture taken using flash. 


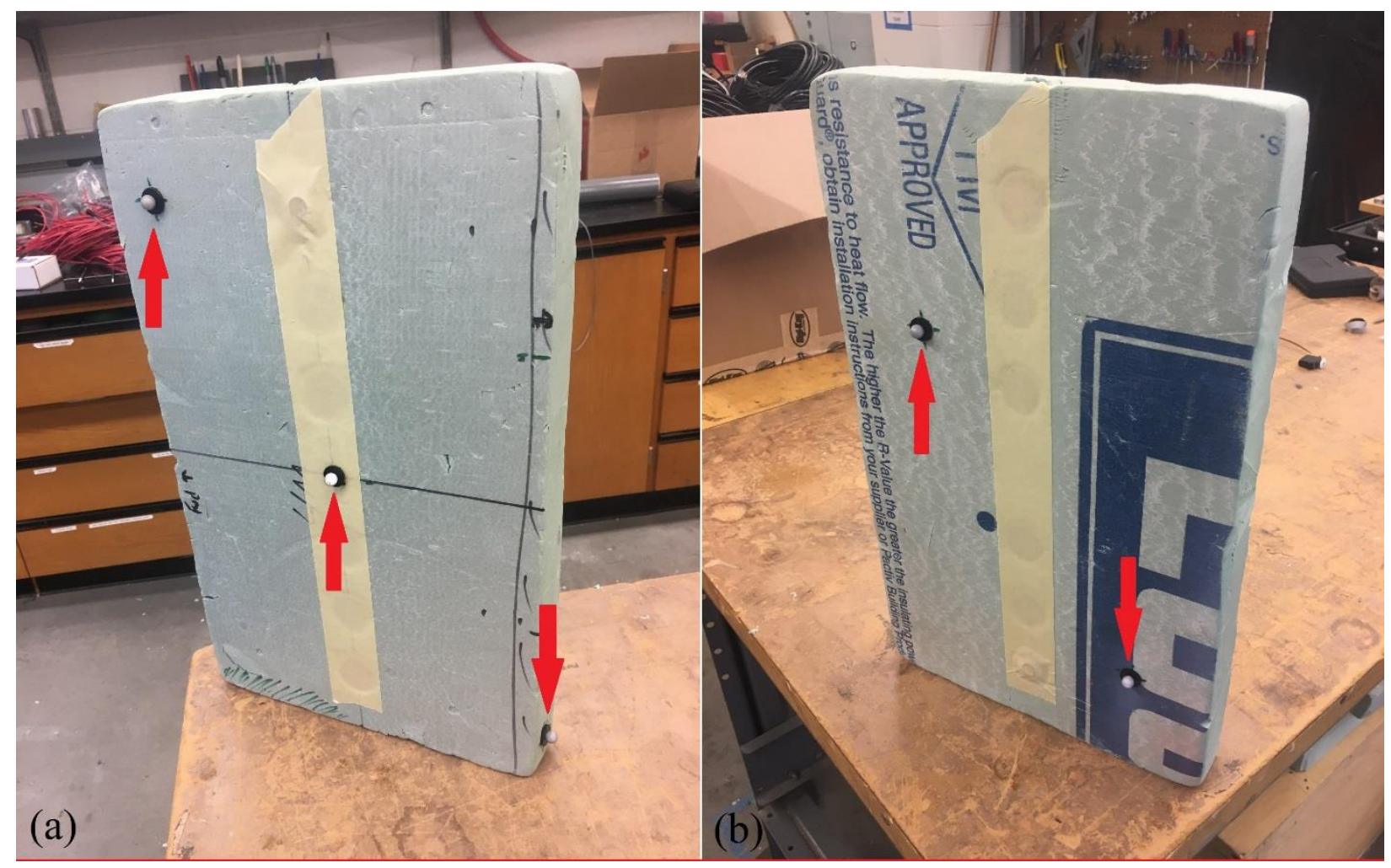

Figure 3.14: (a) Face one of Block 2, which shows three spherical markers indicated by red arrows. (b) Face two of Block 2 which shows two additional spherical markers indicated by red arrows. A total of five spherical markers were used on each block size.

One marker was placed at the center of either face to later determine the center of mass of the block in the software. During object creation, the marker on the center of the block was used to mark the center point for the objects coordinate system. Then, the coordinate system was offset by the distance between two markers on opposing faces. This process allowed the software to calculate quaternion data based on the center of the block. The direction of the axes was also oriented to match the global axes. Figure 3.15 and Figure 3.16 compare a similar perspective of the experimental setup in the real world versus Vicon software. 


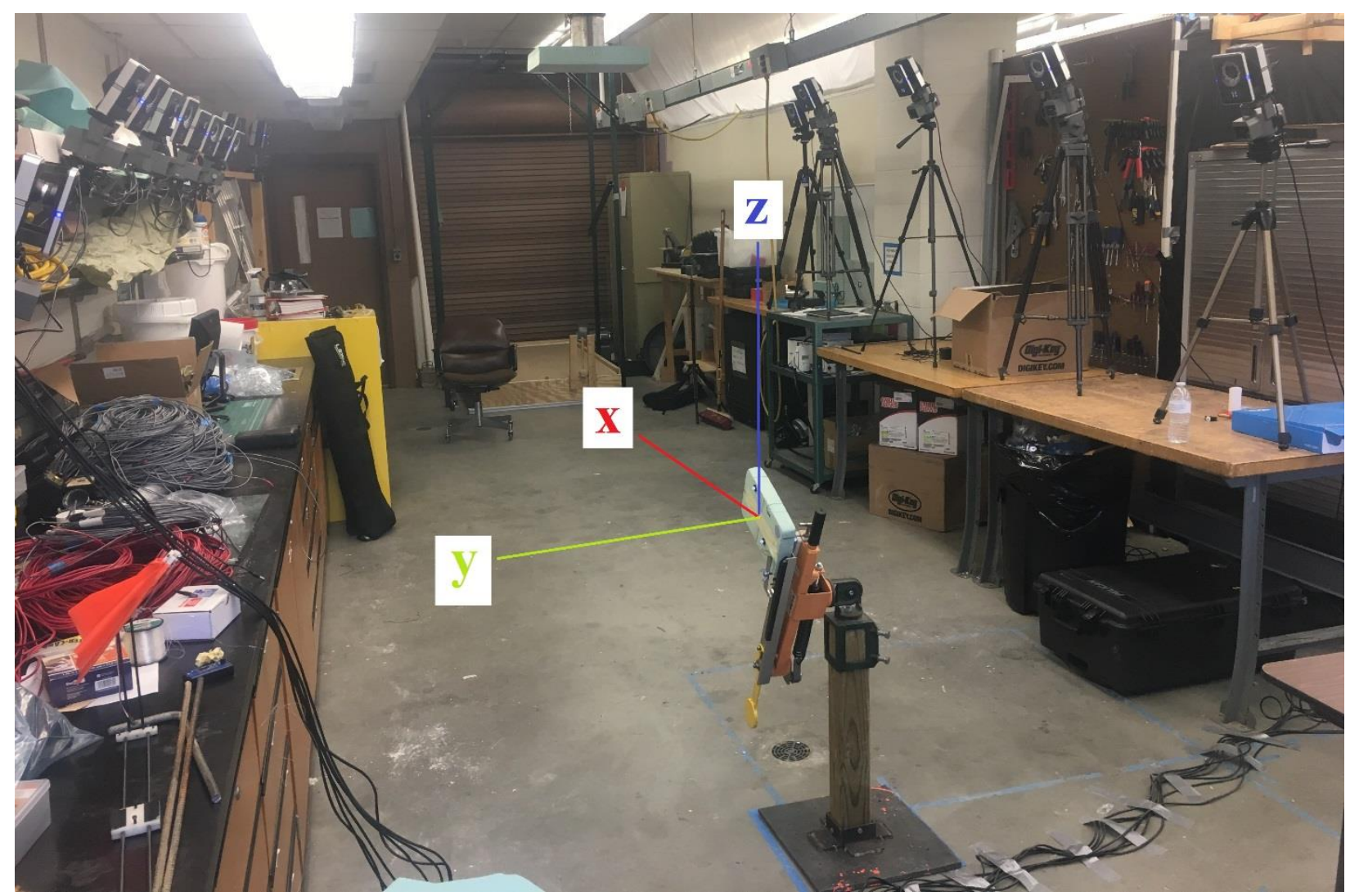

Figure 3.15: Object coordinate system for throwing mechanism evaluated with Vicon camera system.

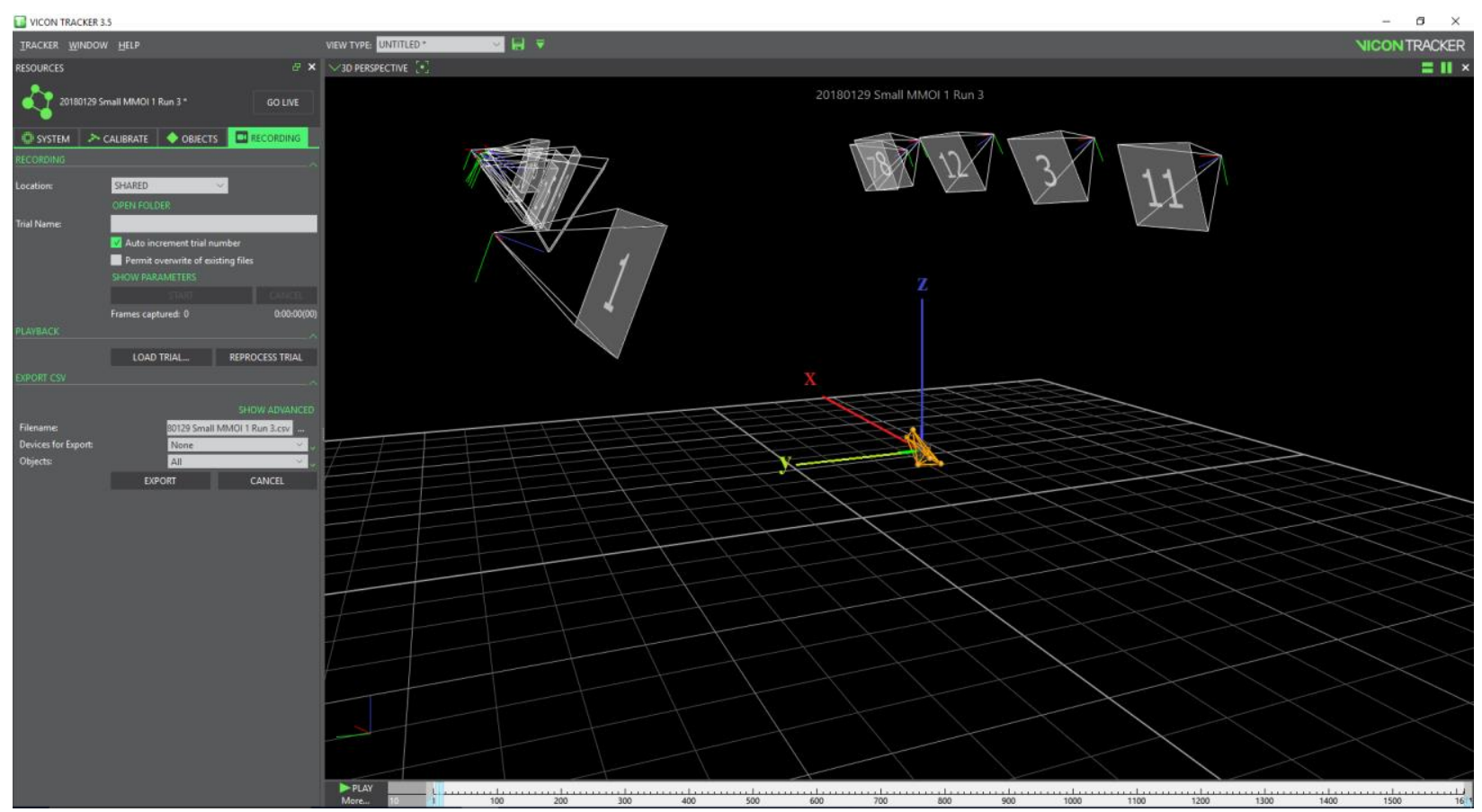

Figure 3.16: Object created in Vicon software based on reflective markers. Coordinate system at CG oriented to match global coordinate system. 
For the purposes of this experiment, five reflective spheres, measuring $9.50 \mathrm{~mm}$ in diameter, were used as the points of interest. Flat, round markers, measuring $32 \mathrm{~mm}$ in diameter, were tested first, due to their relatively lower influence on the aerodynamics of the blocks. However, to create the object, the minimum number of cameras able to "see" the flat markers was not met. For multiple time steps throughout tracking, the object was not created, and no data points were recorded. It was necessary for at least three Vicon cameras to have the marker in frame to create the object. This setting can be changed manually, but having fewer cameras create the object reduces the accuracy. Spheres offered a significant increase in visibility. Since the spheres protruded away from the surface, multiple cameras were able to capture the reflected IR as the block rotated about different axes. Using spheres, aerodynamic effects were increased, compared to the flat markers. However, comparing length scales of the spheres to the blocks shows that the relatively small scale of the spheres should not alter the trajectory a substantial amount. The diameter of the spheres is $9.50 \mathrm{~mm}$, but the spheres were fixed to a small, plastic platform. The addition of the platform increased the total height of the spheres to $10.6 \mathrm{~mm}$ (Figure 3.17). Although, even using the largest length scale of the spherical marker, it was only $4 \%$ of the length scale of Block 1 (Table 3-6).

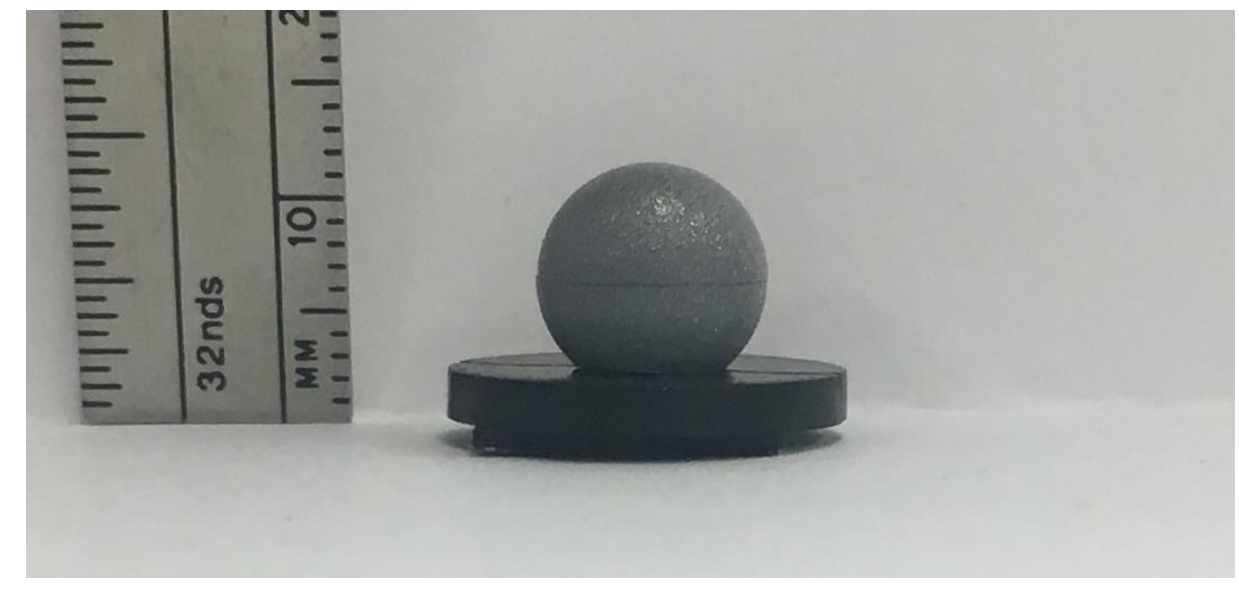

Figure 3.17: Spherical marker length scale of $10.6 \mathrm{~mm}$ from the bottom of the base plate to the top of the sphere. 
Table 3-6: Comparison of block length scales to the spherical marker length scale of $10.6 \mathrm{~mm}$ from the surface of the block.

\begin{tabular}{|c|c|c|}
\hline Test Article & Test Article Length Scale [cm] & Percent of Length Scale \\
\hline Block 1 & 25.4 & $4 \%$ \\
\hline Block 2 & 50.8 & $2 \%$ \\
\hline Block 3 & 61.0 & $2 \%$ \\
\hline Block 4 & 38.0 & $3 \%$ \\
\hline
\end{tabular}

It was also important to track the throwing mechanism. Since the thrower used variable spring tension and launch angle, the transfer of energy to the blocks could vary with adjustments. The same reflective spheres were used on the throwing mechanism to determine its angular velocity throughout its travel. The maximum angular velocity of the thrower during testing with the Vicon system is shown in Table 5-23.

\section{Chapter 4 Testing}

\subsection{SA5 High Speed Camera}

The high-speed camera used to capture foam block trajectory was the FASTCAM SA5 by Photron. At the highest resolution of $1024 \times 1024$ pixels, the camera is able to capture full resolution images at 7,500 fps. However, the highest capture rate used during testing was $500 \mathrm{fps}$. At this setting, the tracker software was easily able to determine the points placed on the foam blocks. The lens used was a $24-85 \mathrm{~mm}$ Nikon with an aperture range from 2.8 to 22 . At $500 \mathrm{fps}$, two to three additional lights, as well as a fully opened aperture, were required to produce a bright enough video for analysis in Tracker. At lower fps, such as 60, the aperture was adjusted to a lower setting based on how much additional lighting was used. To capture the highest field of view, the lens was adjusted to $18 \mathrm{~mm}$ for testing. For drop tests, the camera angle and position were easily altered to capture the entire path of the foam. Alternatively, for the horizontal tests, camera position was limited by the fixed mount location above the table. Therefore, the lens could only capture approximately six feet of the table length. A lens with a wider field of view could have been used, but one consequence of a large field of view is higher distortion at the edge of the frame. While slight distortion can be corrected using an image calibration, high amounts of distortion are not as rudimentary to correct and can cause inaccuracies in data. 


\subsection{Air Table}

The basic design plan was to create a camera mount over a box with a large inlet for air and small, evenly spaced holes across the top surface for the air to exit as free jets (Figure 4.1). As the box reaches a higher pressure than local atmospheric pressure, air will pass through the small holes on the surface as a free jet. The multiple jets will keep the foam block elevated off the surface, allowing it to glide along the surface of the box with minimal friction.

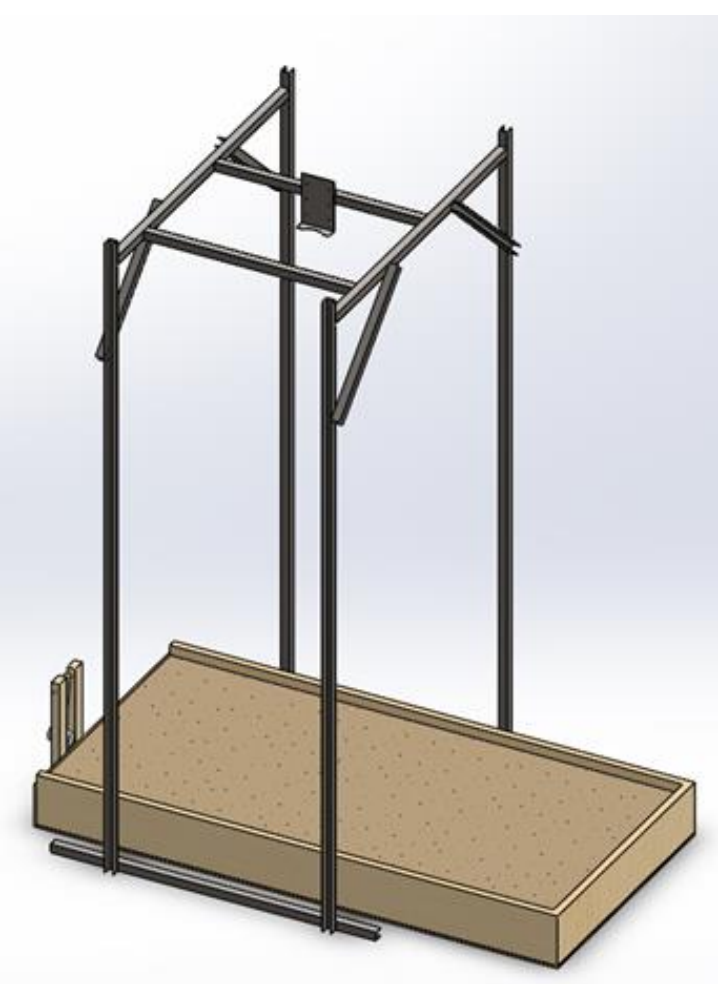

Figure 4.1: Air table, firing mechanism, and Unistrut camera mount.

Design of a suitable air table for testing went through many iterations. Piping the air in from the bottom of the table would cause high differences in air flow through the holes directly above the inlet pipes as compared to those not directly above the inlet. Therefore, the inlet for the table was designed to be located perpendicular to the outlet holes. Another important factor to consider was rigidity of the surface. Since the inside of the table was pressurized, it was important to have a surface that would not deform. A rigid material also created a level surface, critical for accurate trajectories.

Size of the table will vary based on size of the test article. For the purposes of this experiment, the air table was constructed to have a surface 94.5 inches long by 45 inches wide. 
The table size was designed to allow the largest block, Block 3, to spin without interference from the walls. The length of the table was determined by factors such as focal length of the camera lens, ceiling height, optional sizes for surface material, and, most importantly, time for test article to produce accurate data. For example, a shorter table would not allow the block to accelerate to its maximum velocity before interference with one of the three walls. A longer table would require a more powerful blower to produce enough pressure to lift the block off the surface. A longer table would also require a higher camera mount to capture the motion in a 2D plane. As stated previously, due to limitations, only six feet of the table length was captured by the high-speed camera.

The table was constructed using materials shown in Table 4-1. Using a router, a 0.875 inch slot was cut in each board to allow the Medium-density Fiberboard (MDF) to form a tight fit. Seven horizontal cross braces were added to support the weight of the MDF and act as a connection point for metal wire below the surface. The metal wire was designed to hold a layer of insulating material to further distribute the flow of air through the holes if necessary. However, after testing, it was found that the sublayer greatly restricted the air flow. However, from observation, the absence of the insulating layer did not adversely affect the uniformity of the flow (Figure 4.2).

\begin{tabular}{|c|c|}
\hline Part & Quantity \\
\hline $2 " \times 10 " \times 88^{\prime}$ & 3 \\
\hline $4^{\prime} \times 8^{\prime} \times 3 / 4^{\prime \prime} \mathrm{MDF}$ & 1 \\
\hline $4^{\prime} \times 8^{\prime} \times 1 / 4 "$ plywood & 1 \\
\hline $2 " \times 4 " \times 8$ ' & 3 \\
\hline silicone caulk & 1 \\
\hline screws & $\mathrm{n} / \mathrm{a}$ \\
\hline
\end{tabular}




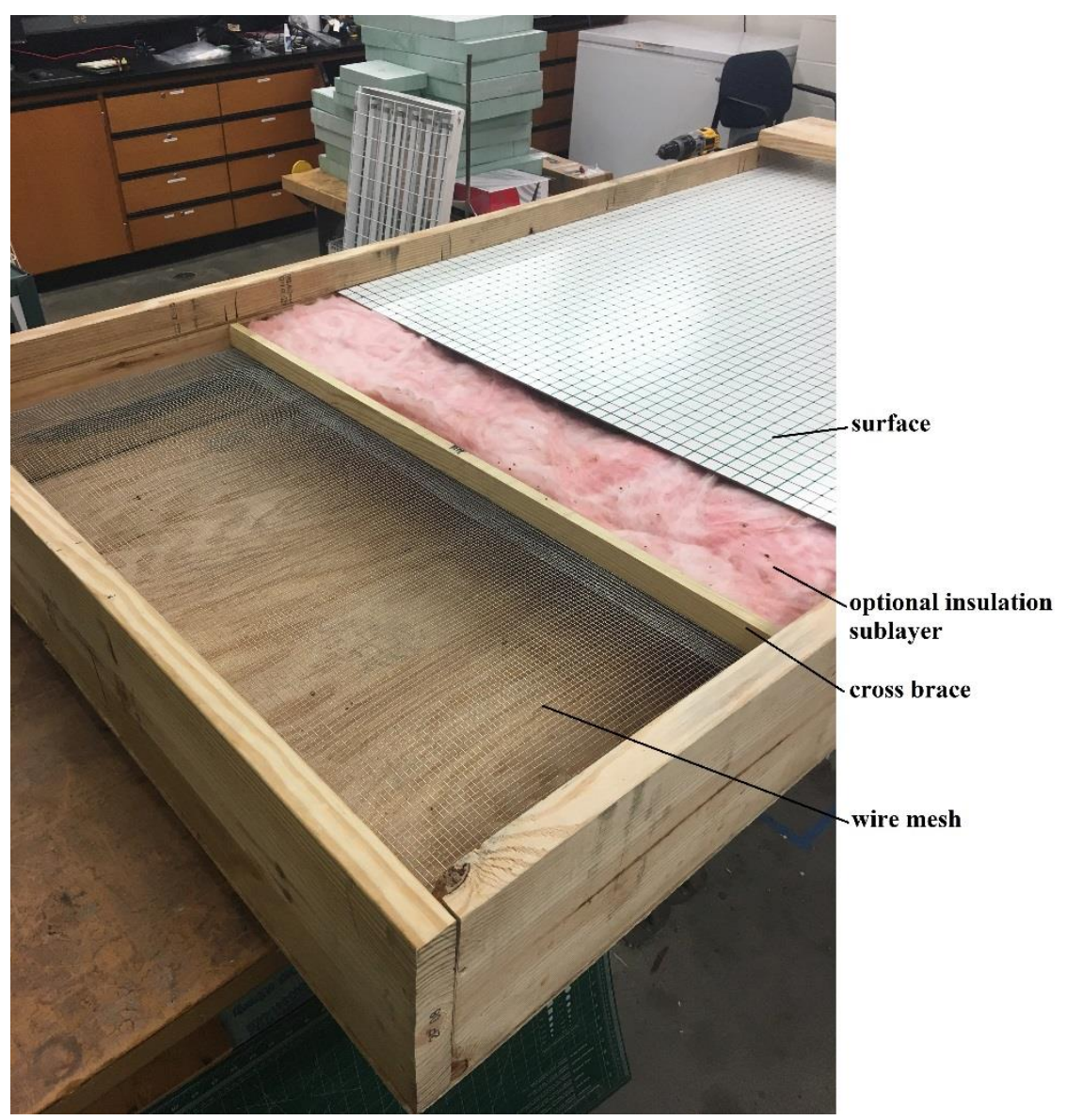

Figure 4.2: Air table anatomy.

To prevent air from escaping through small gaps between the boards, the final design was deconstructed and a bead of silicone caulk was placed between each joint as well as along the creases on the sides of the surface.

The blower fan used for this experiment was a 1 HP Dayton model number 5K901C. The fan was inserted into a square hole cut specifically for the fan's exit vent. Thus, a good seal was created around the vent. Tape held the fan from blowing itself backward as pressure in the table increased (Figure 4.3). 


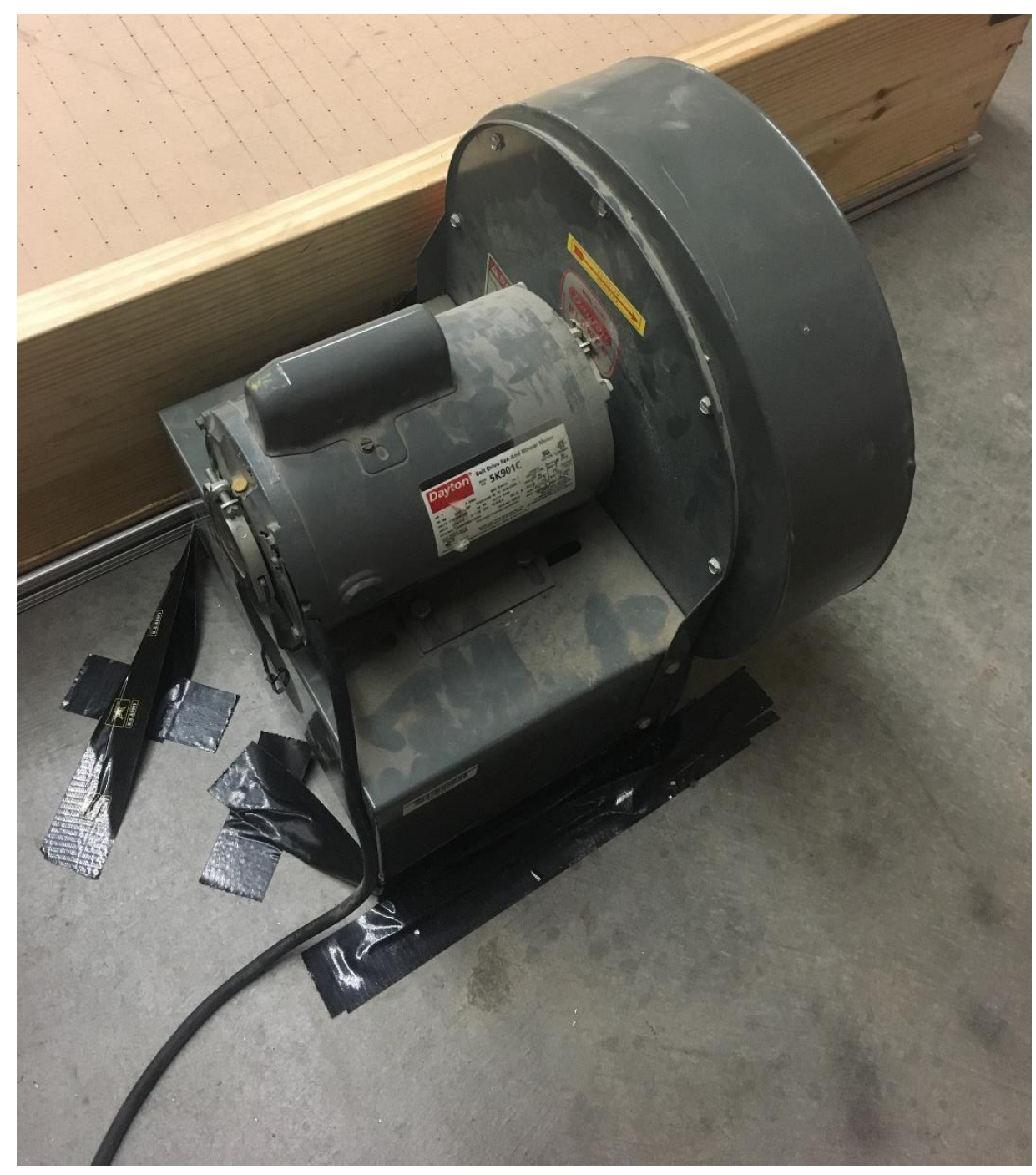

Figure 4.3: Fan used to pressurize the table.

To reduce the effects of gravity on the path of the block, the table was determined to be level for every series of tests that were performed. A 24-inch level was used in each plane of the table in different locations across its span (Figure 4.4). If the table was out of level, thin aluminum shims were inserted for correction (Figure 4.5). 


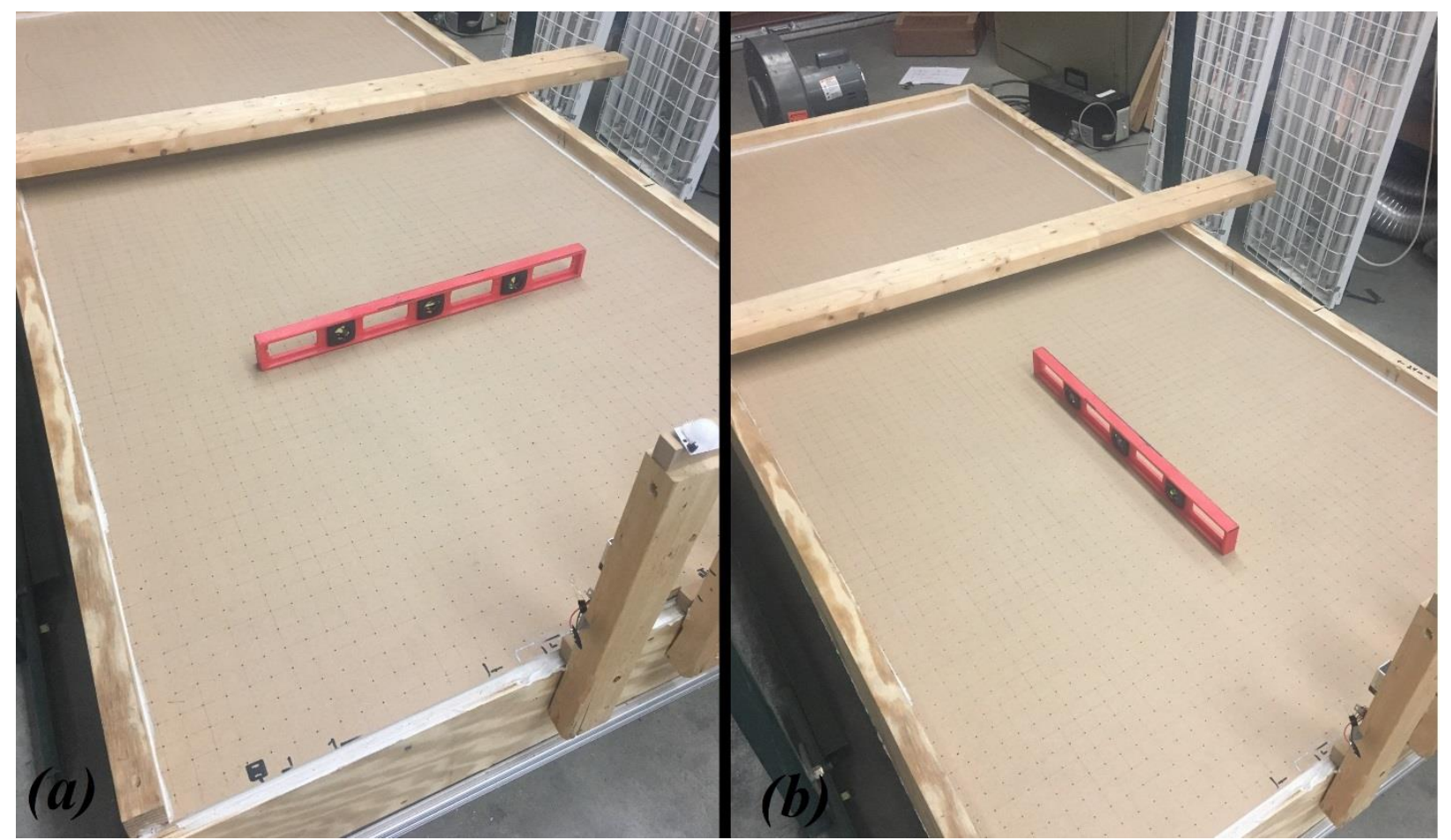

Figure 4.4: Table leveling process.

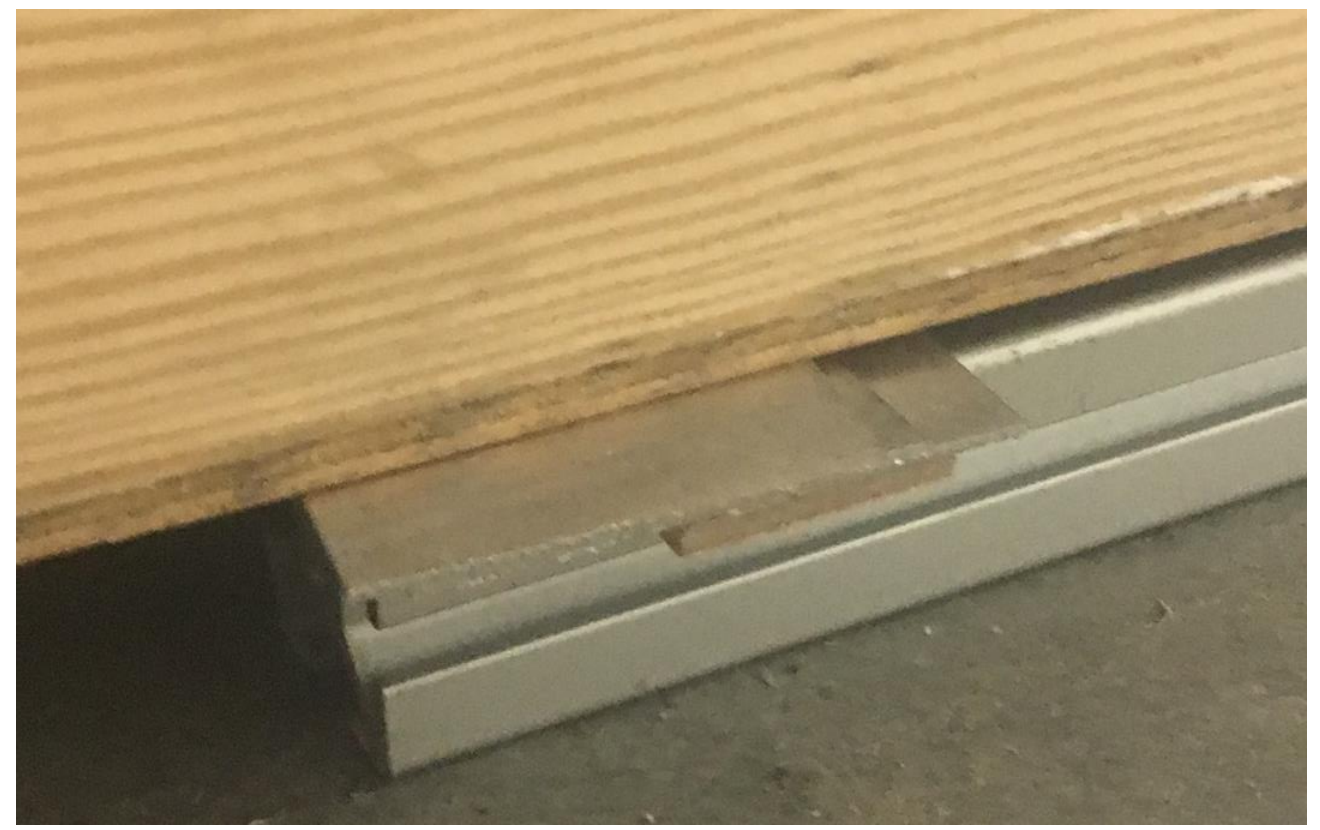

Figure 4.5: Aluminum shims added to level the table.

Additionally, small material such as dust and dirt collected on the surface of the table. To reduce the chance of the block contacting a foreign object, the surface was cleaned before session of tests using a shop broom and, if needed, compressed air. 
The Unistrut frame was designed to be able to safely support the high-speed camera. Mounted above the table, the end of the lens was 96 inches from the surface of the table. At this distance, the camera was able to capture 72 inches of the table's length. The frame was built wide enough to span over the table while not interfering with the firing mechanism or block trajectory. To mount the camera at a 90 degree angle, a custom plate was fabricated (Figure 4.6 and Figure 4.7).
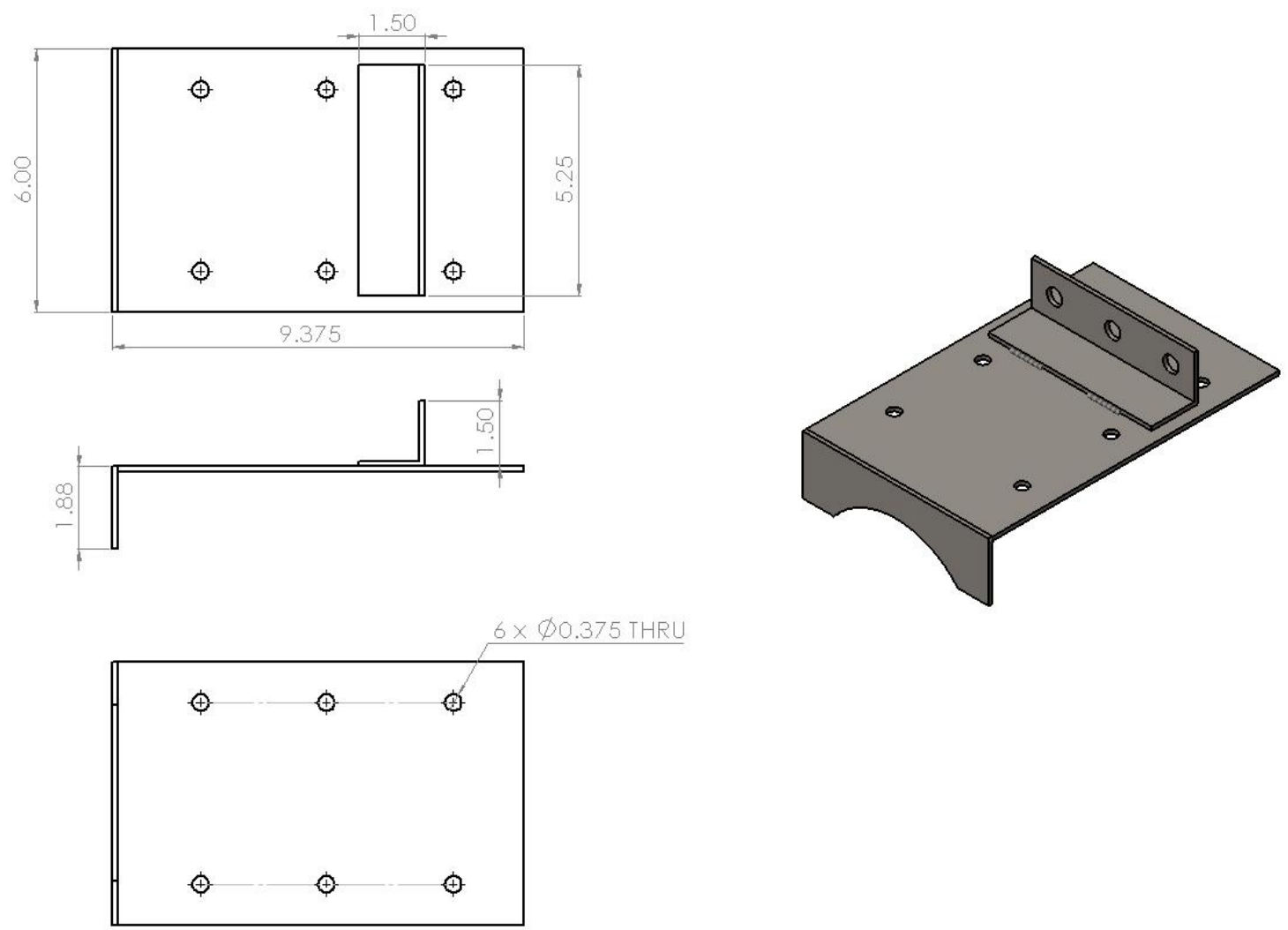

Figure 4.6: CAD model of SA5 high speed camera mount for angling the camera 90 degrees to the air table surface. 


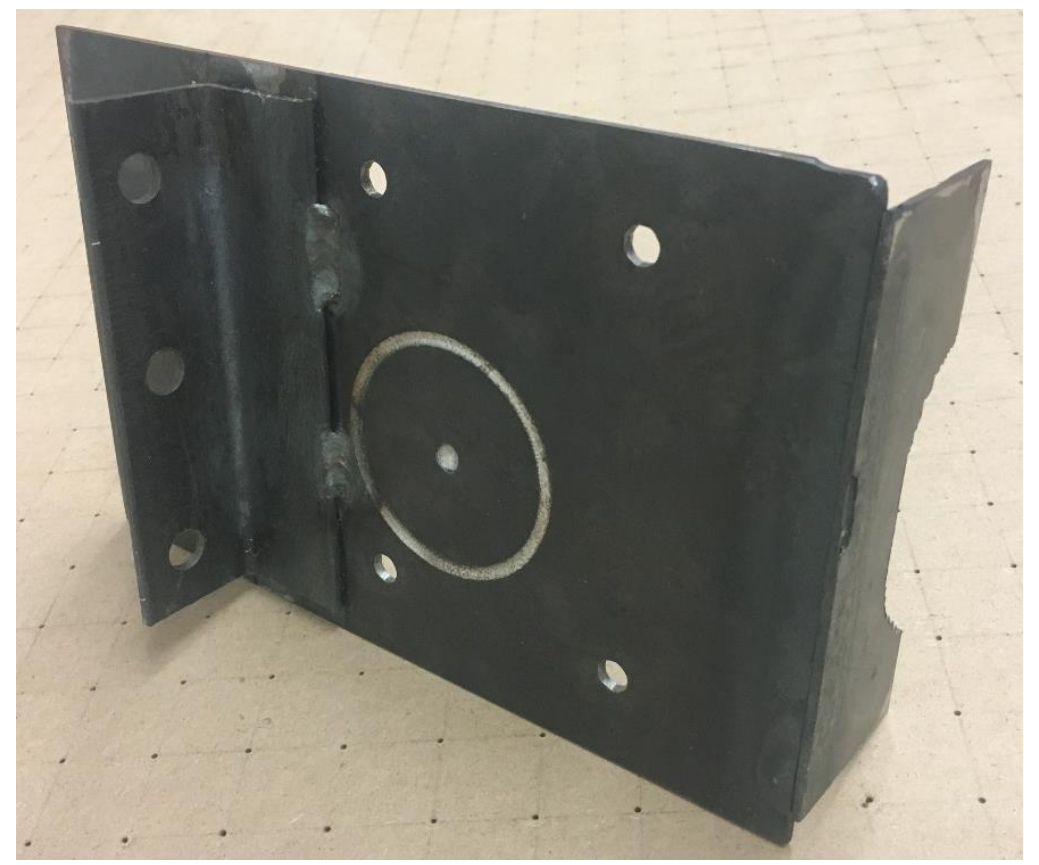

Figure 4.7: Camera mount for horizontal plane experiments.

The camera was mounted to the plate by means of the six M6 bolt holes in the bottom of the camera. Next, the plate was mounted to the Unistrut frame using the three $1 / 2$ inch bolts. Figure 4.8 shows the camera mounted above the table using the custom plate, a safety chain, and a string intended to reduce swing. 

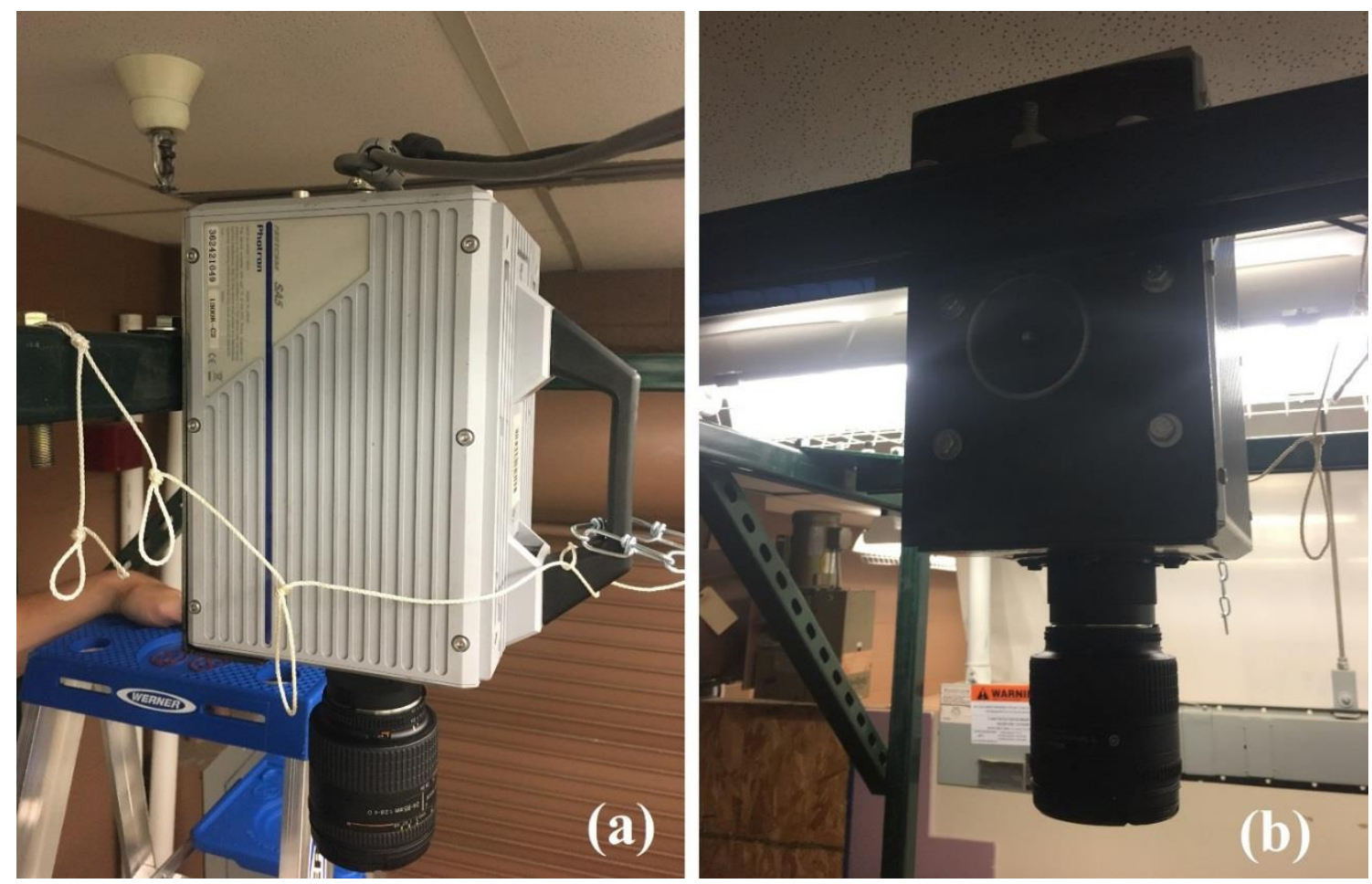

Figure 4.8: Camera mounted above air table during horizontal tests. (a) Side view. (b) Rear view.

The final component of the air table is the firing mechanism. The simplest solution for a repeatable initial force applied to the block was a pendulum. A common mallet was used to hit the block and cause translation and rotation. The hammer needed to be rigid in the plane of its swing to impact the same location on the block for each run. Thus, a small stop block was used to position the foam block in the same location. For the hammer drop height, it was determined that rotating the hammer to an angle of 90 degrees put its head at a suitable height. Calculations for this initial condition are shown in Chapter 5. To ensure a repeatable drop height, a screw was placed at the tip of the hammer mount to stop the hammer when it reached 90 degrees. Figure 4.9 shows the setup of the firing mechanism and how the stop block was used to position the foam block. 


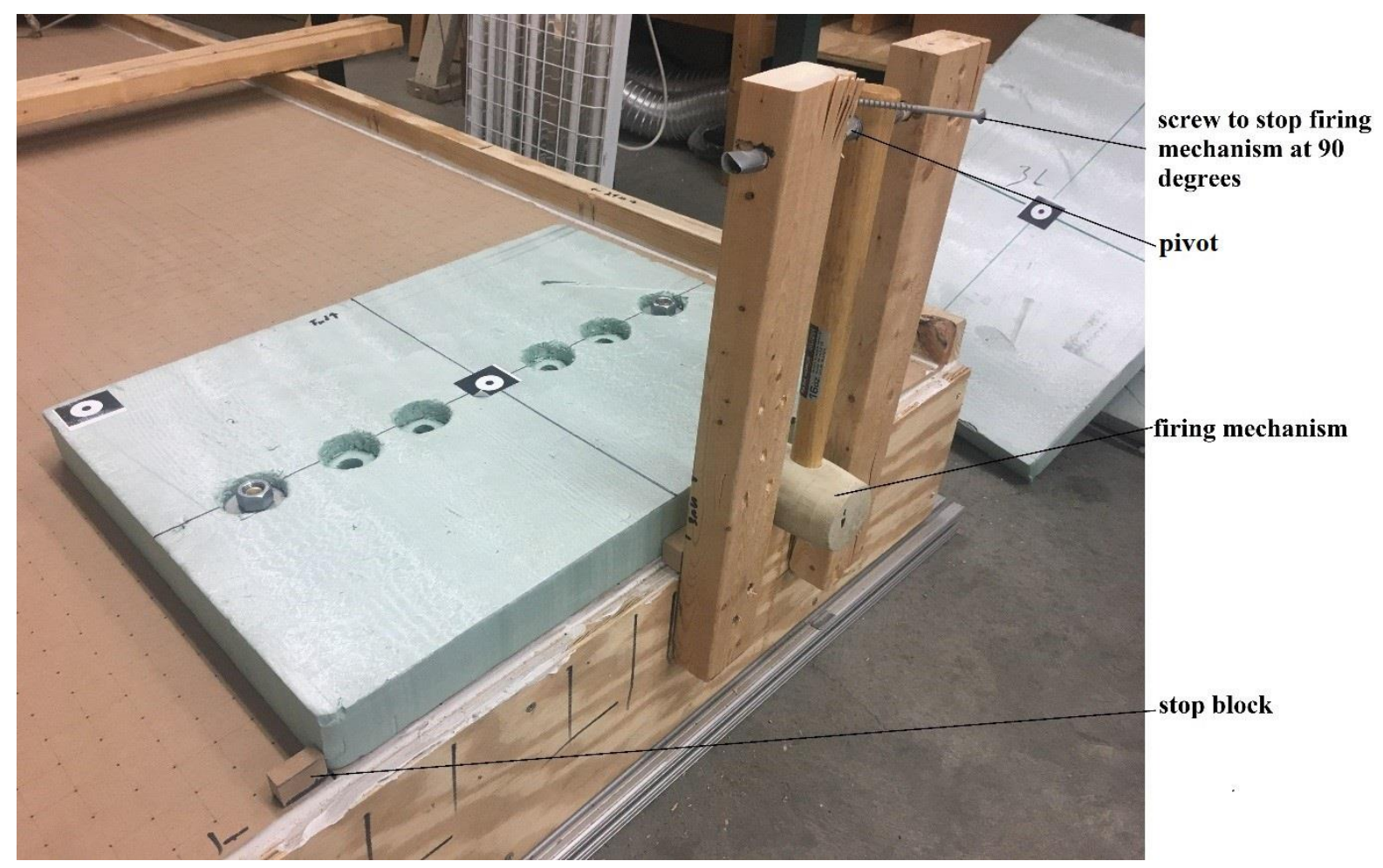

Figure 4.9: Firing mechanism for horizontal tests.

\subsection{Drop Tests}

The drop test allowed the blocks to fall from a height of $243 \mathrm{~cm}$ due to the effects of gravity (Figure 4.10). However, due to imbalances in the desired axis of rotation, the distance of trajectory tracked for each block varied. To determine a velocity and angular velocity, three different methods were used.

The relatively short distance traveled before off-axis rotation did not allow the blocks to reach terminal velocity. Therefore, unlike the other tests conducted, the blocks were constantly accelerating throughout their trajectories.

The only factors changing between tests were MMOI and block size. During the fall, angular and translational velocities of each block for each MMOI were recorded until the tracker markers rotated out of view of the camera. Tracker data was collected for approximately half of the trajectory for most tests. To better understand the relationship between initial conditions and the tendency of the blocks to tumble, a 36"x48" grid of four inch squares was placed on the floor directly below the center of the block, perpendicular to the plane in which the blocks were initially rotating (Figure 4.11). 

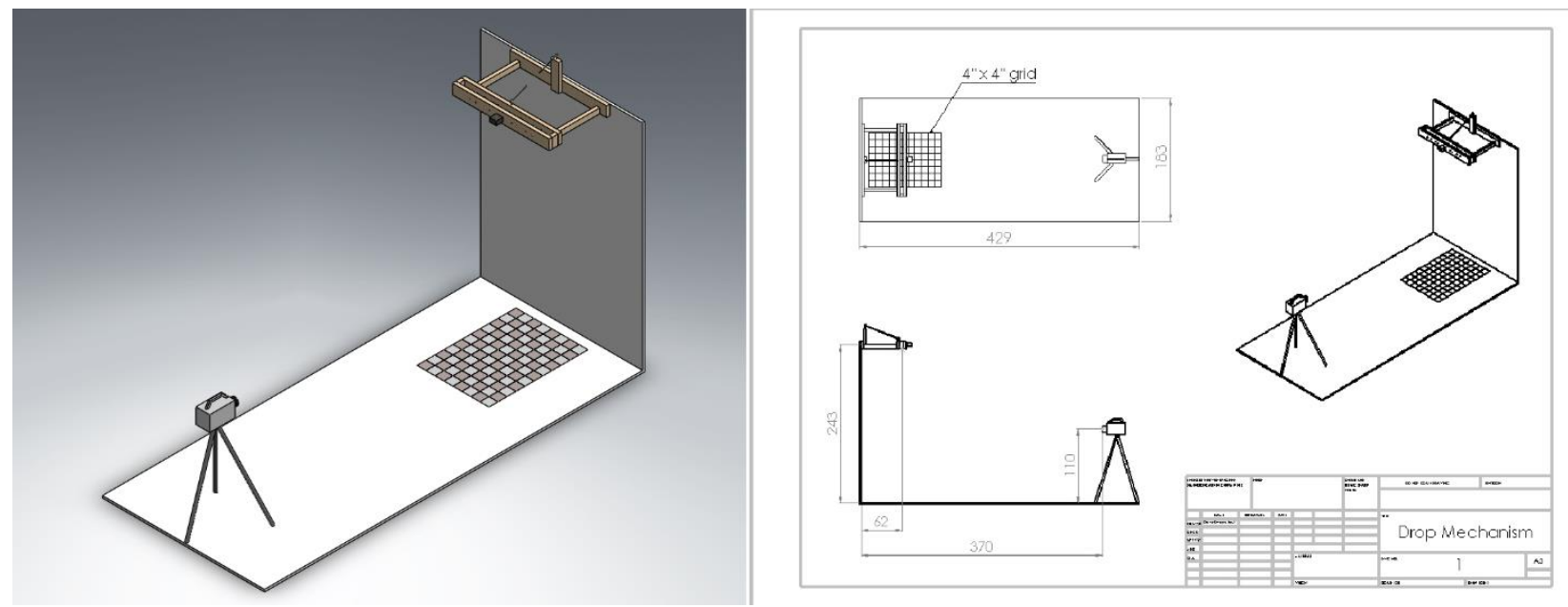

Figure 4.10: CAD and dimensions of drop mechanism experiment. Dimensions are shown in centimeters.

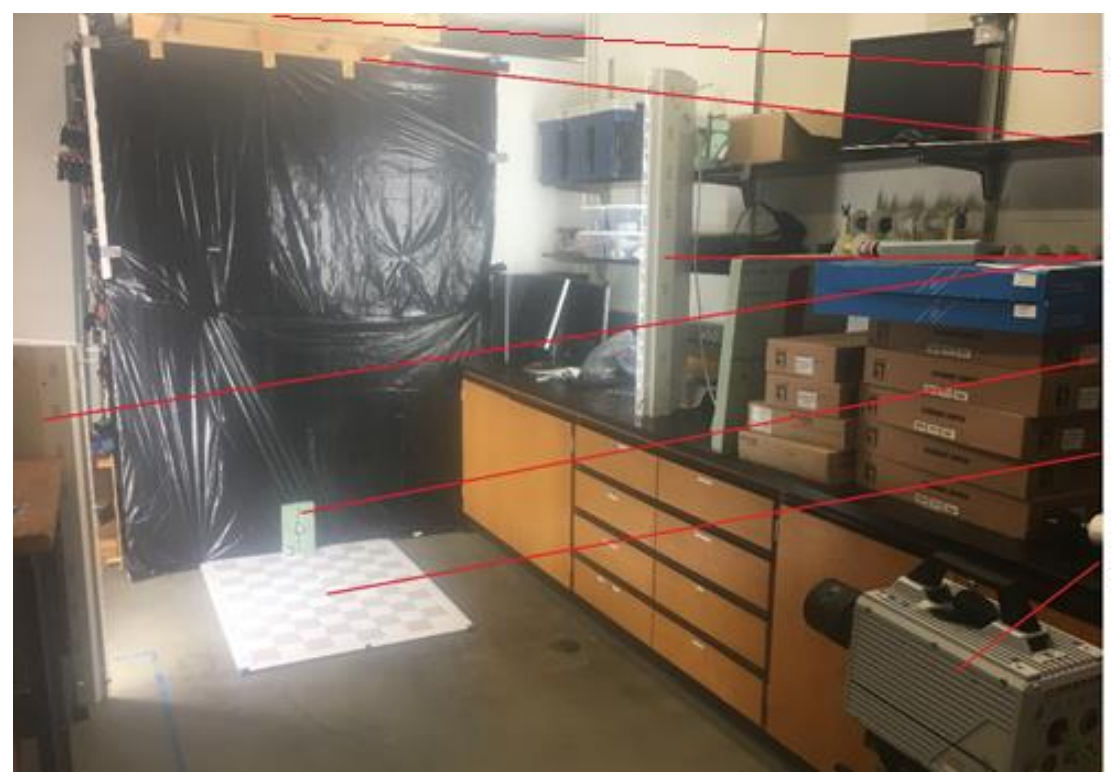

GoPro Camera

Drop Mechanism

Additional

Lighting

Test Article

$36 " \times 48$ " Grid

High Speed

Camera

Figure 4.11: Drop test experiment setup to determine relationship between a relationship between dimensionless MMOI and angular velocity and tendency to tumble into perpendicular plane.

To create repeatable drops, each block was held in place in the drop mechanism by two $1 / 4$ " rods (Figure 4.12). One rod was attached to a string that was pulled quickly to release the block. When one rod is removed, the block pivots about the other rod for approximately 20-30 degrees until the surfaces separate and the block continues to rotate towards the ground. 


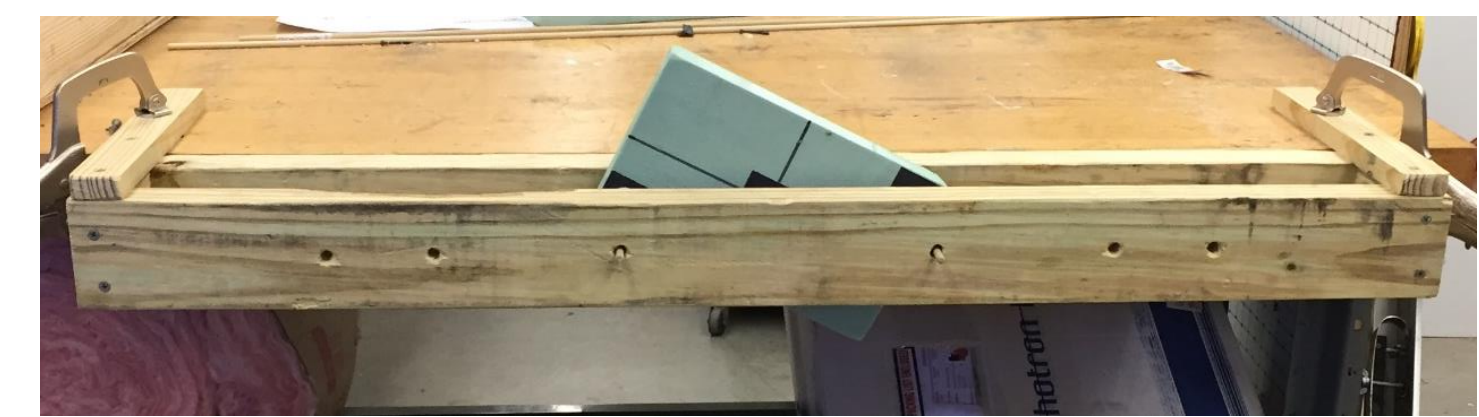

Figure 4.12: Initial condition of block for drop testing. The block is held by two 1/4" rods until one is quickly removed using the attached string.

Three tests were conducted for each of the four blocks for each MMOI. The average measured values of translational and angular velocity for the three runs were compared using plots and an ANOVA analysis to determine a relationship and interaction. See Chapter 5 for trajectory data analysis.

Over the three randomized tests of each size and MMOI, the SA5 camera captured video at $500 \mathrm{fps}$ and 1/1000 shutter speed. A GoPro Hero 3 was placed on the drop mechanism perpendicular to the high-speed camera. The GoPro camera was used to reference the location of the block's center of mass over the grid as it impacted the ground. The block may impact the ground in any orientation. During impact, both the high-speed camera and the GoPro were used to determine which square on the grid contained the center of mass. The grid used is shown in Figure 4.13. The center is marked with a black dot for reference. Data determined using the grid is shown in Table 4-2, while a visual representation can be seen in Figure 4.14. The landing locations are based on the two perspectives showing in Figure 4.15 (Block 2 with MMOI 1 is used as an example). As shown in Figure 4.15, the block did not necessarily impact the ground horizontally; the location was recorded during the first impact of the ground, regardless of orientation of the block. 


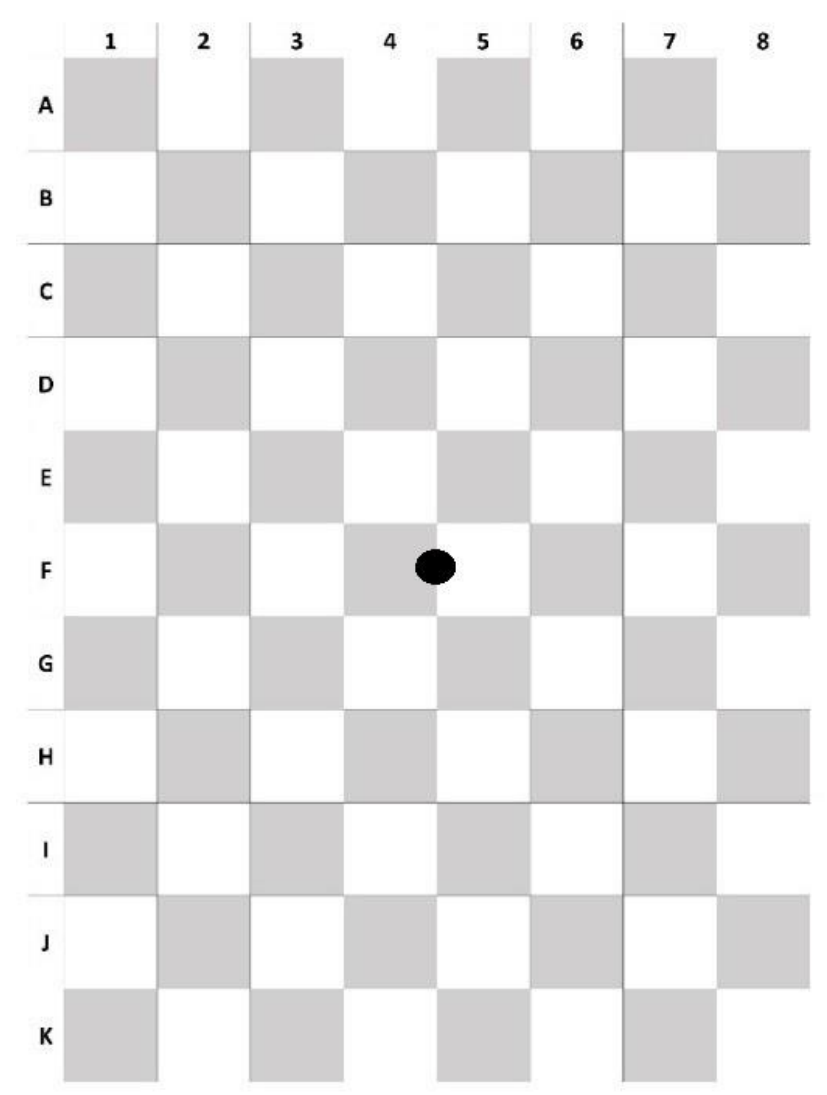

Figure 4.13: Grid of 4"x4" squares used to determine horizontal block motion. The center of the grid, signified using a block dot, was aligned with the CG of the block usign a plumb bob.

Table 4-2: Impact locations of specimens of different size and MMOI.

\begin{tabular}{|c|l|c|c|c|}
\hline & & $\begin{array}{c}\text { Run } \\
1\end{array}$ & $\begin{array}{c}\text { Run } \\
2\end{array}$ & $\begin{array}{c}\text { Run } \\
3\end{array}$ \\
\hline Block 1 & MMOI 1 & H6 & H7 & D7 \\
\cline { 2 - 5 } & MMOI 2 & H7 & H7 & D6 \\
\cline { 2 - 5 } & MMOI 3 & G7 & H7 & H7 \\
\hline Block 2 & MMOI 1 & F5 & H6 & G5 \\
\cline { 2 - 5 } & MMOI 2 & E5 & I5 & I6 \\
\cline { 2 - 5 } & MMOI 3 & I6 & H6 & E6 \\
\hline Block 3 & MMOI 1 & G5 & G5 & H5 \\
\cline { 2 - 5 } & MMOI 2 & G5 & H6 & H5 \\
\cline { 2 - 5 } & MMOI 3 & G5 & E5 & G6 \\
\hline Block 4 & MMOI 1 & H5 & H6 & H6 \\
\cline { 2 - 5 } & MMOI 2 & G5 & H6 & G6 \\
\cline { 2 - 5 } & MMOI 3 & H6 & I6 & E6 \\
\hline
\end{tabular}


The data collected in Table 4-2 was used to determine effects of MMOI and geometry on each block's motion in the horizontal plane. Blocks were placed in the drop mechanism in the same orientation for each run, but direction of the additional weights was randomized, along with order of test article dropped, to reduce any bias that may have been caused by unbalanced weights. It was assumed that any inconsistent impact locations were caused by the change in MMOI or size of the block, but other factors, such as wall effects, damage to the block, and block imbalance could also influence impact location.

\begin{tabular}{|c|c|c|c|c|c|c|c|c|c|}
\hline & 1 & 2 & 3 & 4 & 5 & 6 & 7 & 8 & \multirow[b]{3}{*}{$\begin{array}{lll}\Delta & \text { Block } 1\end{array}$} \\
\hline A & & & & & & & & & \\
\hline B & & & & & & & & & \\
\hline C & & & & & & & & & (- Block 2 \\
\hline D & & & & & & $\Delta$ & $\Delta$ & & Dt Block 3 \\
\hline $\mathrm{E}$ & & & & & 2 & 3 & & & Block 4 \\
\hline $\mathrm{F}$ & & & & & 0 & & & & \\
\hline G & & & & & 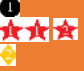 & & A & & \\
\hline $\mathrm{H}$ & & & & & 사에 & $\begin{array}{l}A \Delta A \\
A O \\
A O\end{array}$ & $\triangle \mathrm{AA}$ & & \\
\hline 1 & & & & & $\overline{8}$ & 83 & & & \\
\hline $\mathrm{J}$ & & & & & & & & & \\
\hline K & & & & & & & & & \\
\hline
\end{tabular}

Figure 4.14: Visual representation of the impact locations of test articles. Numbers represent MMOI of the block while shape and color of represent size. (E.g. a blue triangle containing the number 1 represents the Block 1 with MMOI 1). 

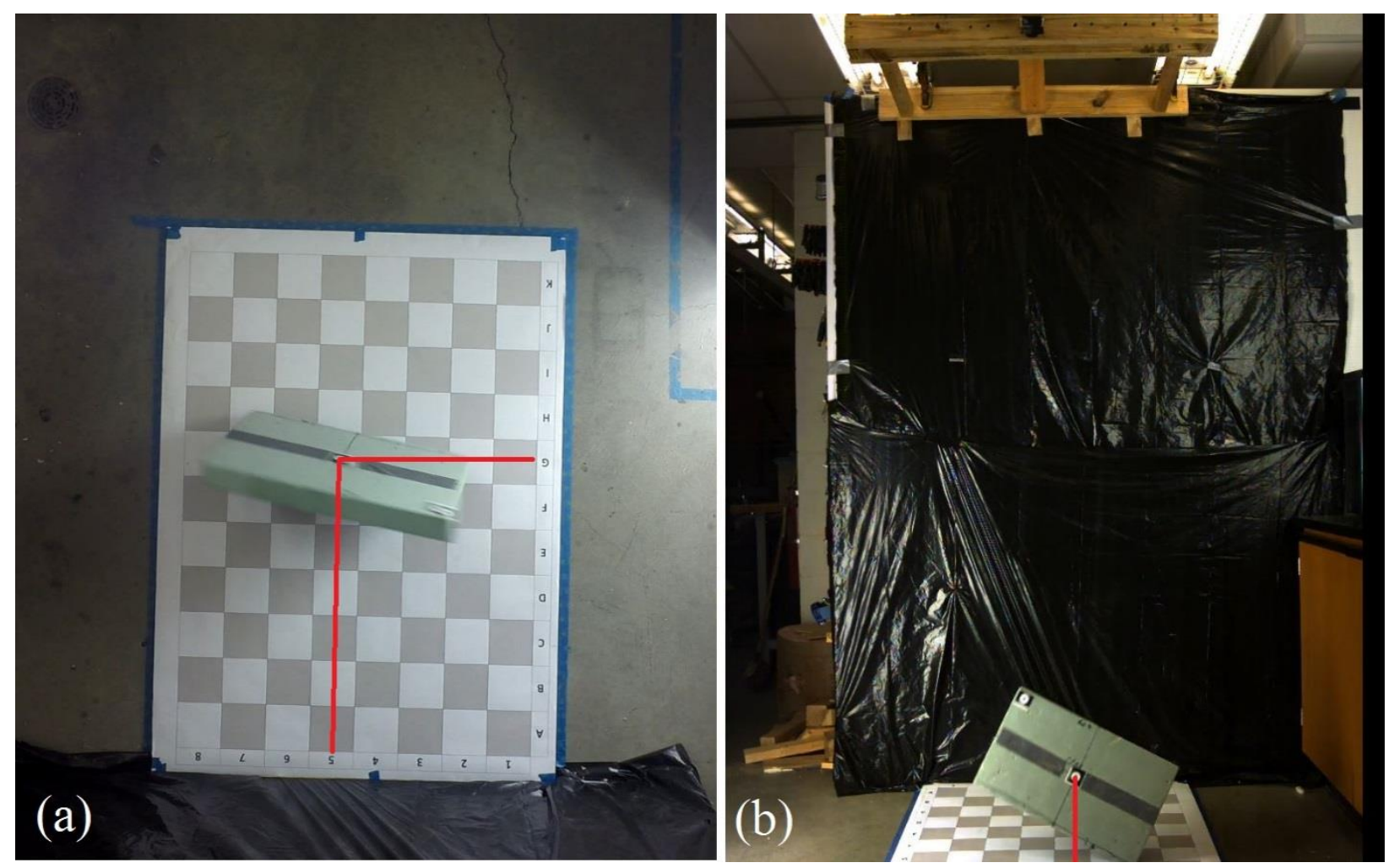

Figure 4.15: (a) GoPro perspective used to determine block location at impact in the horizontal plane. (b) High speed camera perspective used to determine block location at impact in the vertical plane. Block 2 with MMOI 1 is used as an example. The location for this example is G5 and can cross referenced in Figure 4.14, showing all drop locations.

Based on data shown in Figure 4.14, impact locations of the blocks demonstrate relatively close grouping. Slight differences in impact location, such as with the Block 1 - MMOI 1, are assumed to be caused by imperfections in the block or mounting of the block. A significant change between impact locations would be accredited to MMOI or size change if one group tended to land in a location away from the other grouping of impacts. The ANOVA analysis performed on the data shows the effects of MMOI and geometry on landing location. That is not to say that, given a longer distance for which to fall, the blocks would not deviate from the average more than depicted in the figure. Thus, for a height of 243 centimeters, no effects can be determined from coordinates alone. The ANOVA analysis conducted on the data, Table 5-21, further investigates the influence of MMOI and size on impact location.

\subsection{Throwing Mechanism SA5 High Speed Camera}

Using a clay pigeon thrower (Figure 4.16), most commonly used in sport shooting, the blocks were thrown through means of initial energy stored within the spring. The blocks were placed in an upright position to allow a similar amount of contact with the throwing arm. As the 
arm was released, the block gained translational and rotational energy. The rotational velocity gained by the block was measured to be an order of magnitude higher than that of the air table test. The large range of angular velocities tested allow for a more accurate scaling analysis. The SA5 high speed camera was used to capture each block's trajectory as it traveled approximately three meters in the horizontal axis before being caught in a plastic sheet.

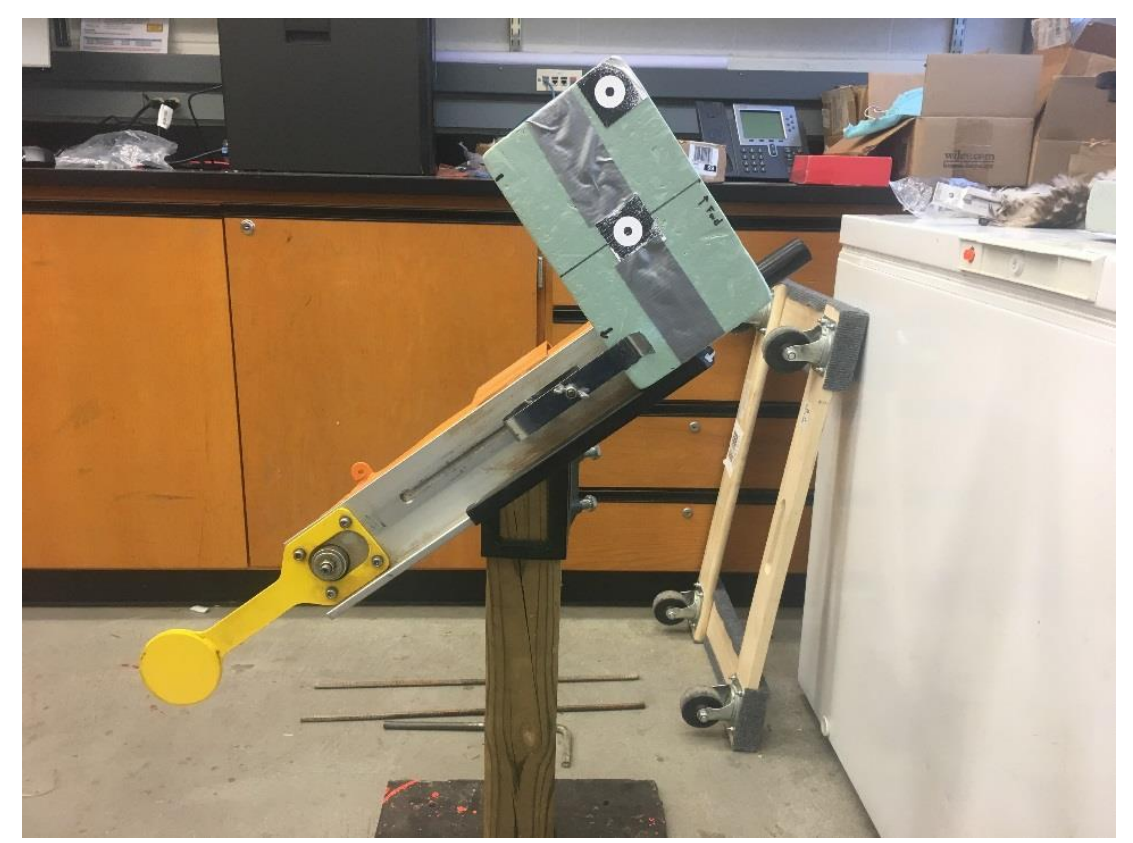

Figure 4.16: Clay pigeon thrower used to induce rotational and translational motion. Note that the midpoint of the block in the " $L$ " dimension is aligned with the end of the holder.

A CAD representation of the experimental setup is shown in Figure 4.17. Note that the distance of 130 inches captured by the SA5 high speed camera is significantly smaller than that of the distance captured by the Vicon system, discussed section 4.5 . 

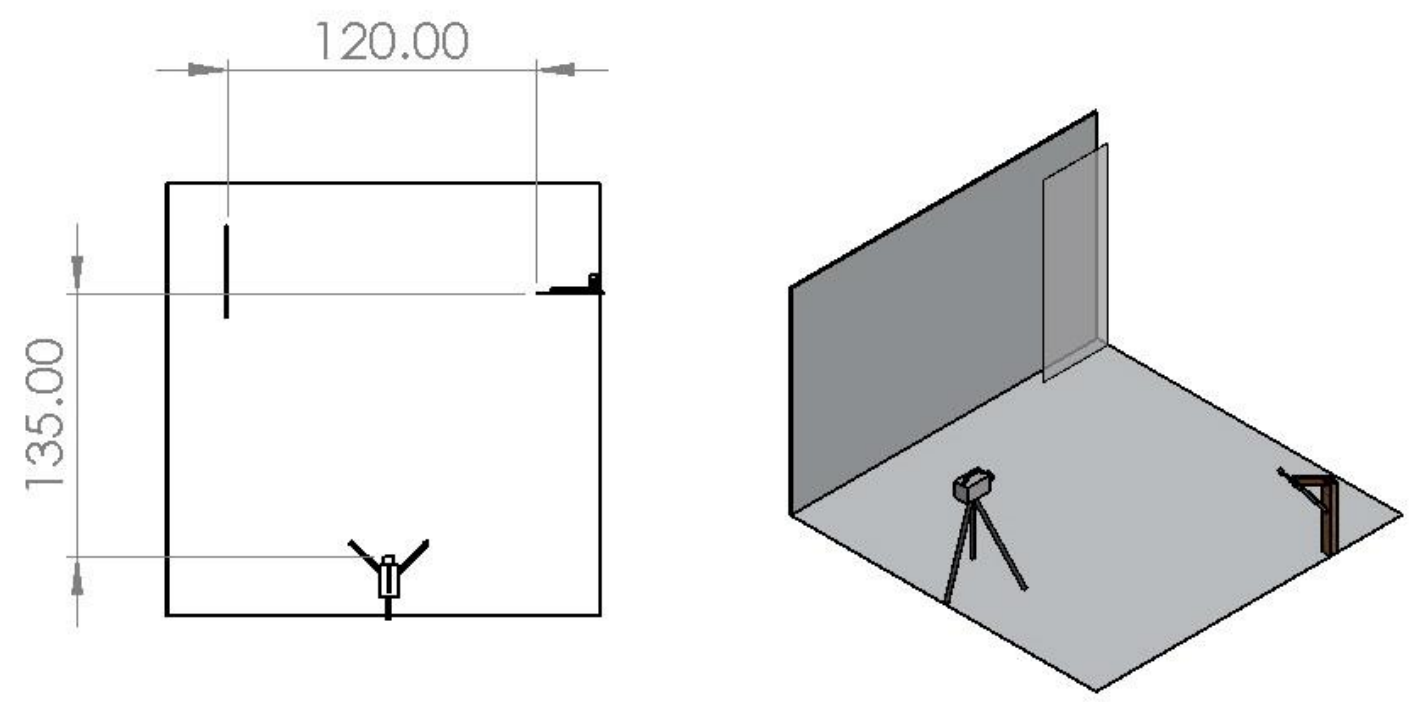

Figure 4.17: Throwing arm test experimental setup. Dimensions given in inches.

Each block was held in the throwing mechanism using the adjustable tension arm. The initial angle of the throwing mechanism was governed by field of view of the camera lens. Table 4-3 shows the initial throwing arm angles used for each block size. It was desired to have the block travel through the entire field of view. Therefore, the arm was angled differently for the smaller block to keep it within the field of view. When the smaller block was initially tested using the same angle as the other block sizes, its trajectory tended to follow a more vertical path, rather than horizontal. Simply, at an angle of 53 degrees, the small block would be fired toward the ceiling, rather than into the damping sheet. The angles were calculated using a level and protractor as demonstrated in 4.18. Inspecting the angle by eye increased the probability of human error. However, the clay pigeon thrower used was equipped with "no-slip" textured plates to aid in angle adjustments. By mating the textured plates together as the nut was tightened, the thrower was held at a specific angle. Each groove in the plate corresponded to an adjustment of approximately 9 degrees. Figure 4.19 shows the textured plates, and how their interaction held the arm at a fixed angle. 


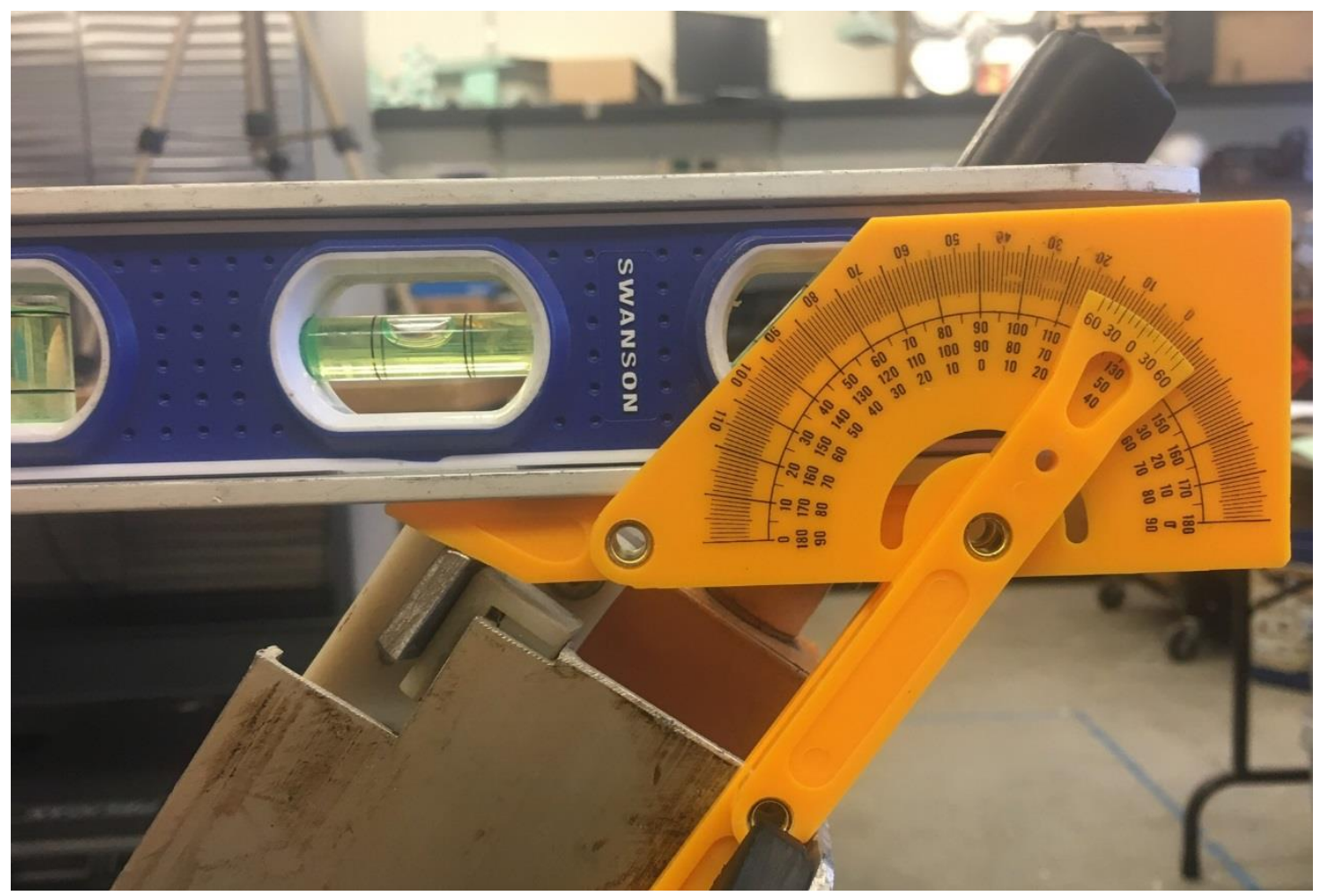

4.18: Initial thrower arm calculation using level and protractor.

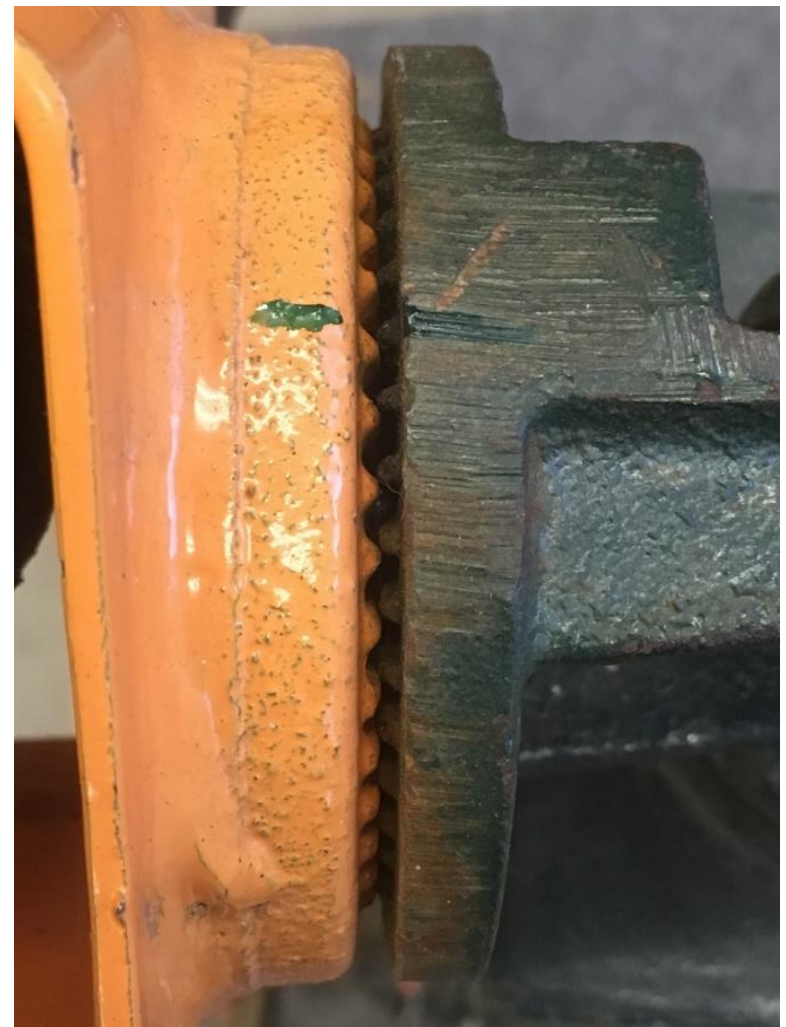

Figure 4.19: Throwing mechanism angle adjustment plate locking system. The plates were slightly loosened to better show the ridge interaction. 
Table 4-3: Initial thrower arm angle to induce a trajectory trackable by the SA5 camera system.

\begin{tabular}{|c|c|}
\hline Size & Angle [deg] \\
\hline Block 1 & 62 \\
\hline Block 2 & 53 \\
\hline Block 3 & 53 \\
\hline Block 4 & 53 \\
\hline
\end{tabular}

For each MMOI, three tests were conducted for each of the four blocks. The average measured values of translational and angular velocity for the three runs were compared using plots and an ANOVA analysis to determine the existence of a scaling relationship.

\subsection{Throwing Mechanism VICON Camera System}

The Vicon camera system used a collection of cameras to measure differences in grayscale within the frame and circle fitting the markers. 12 Vicon cameras were placed in two rows down a designated volume, $518 \mathrm{~cm}$ in the $x$-axis, $274 \mathrm{~cm}$ in the $y$-axis, and approximately $196 \mathrm{~cm}$ in the $z$-axis, measured from the block's CG as it rested in the launcher (Figure 4.20). Figure 4.21 shows the experimental setup with the coordinate system overlaid.

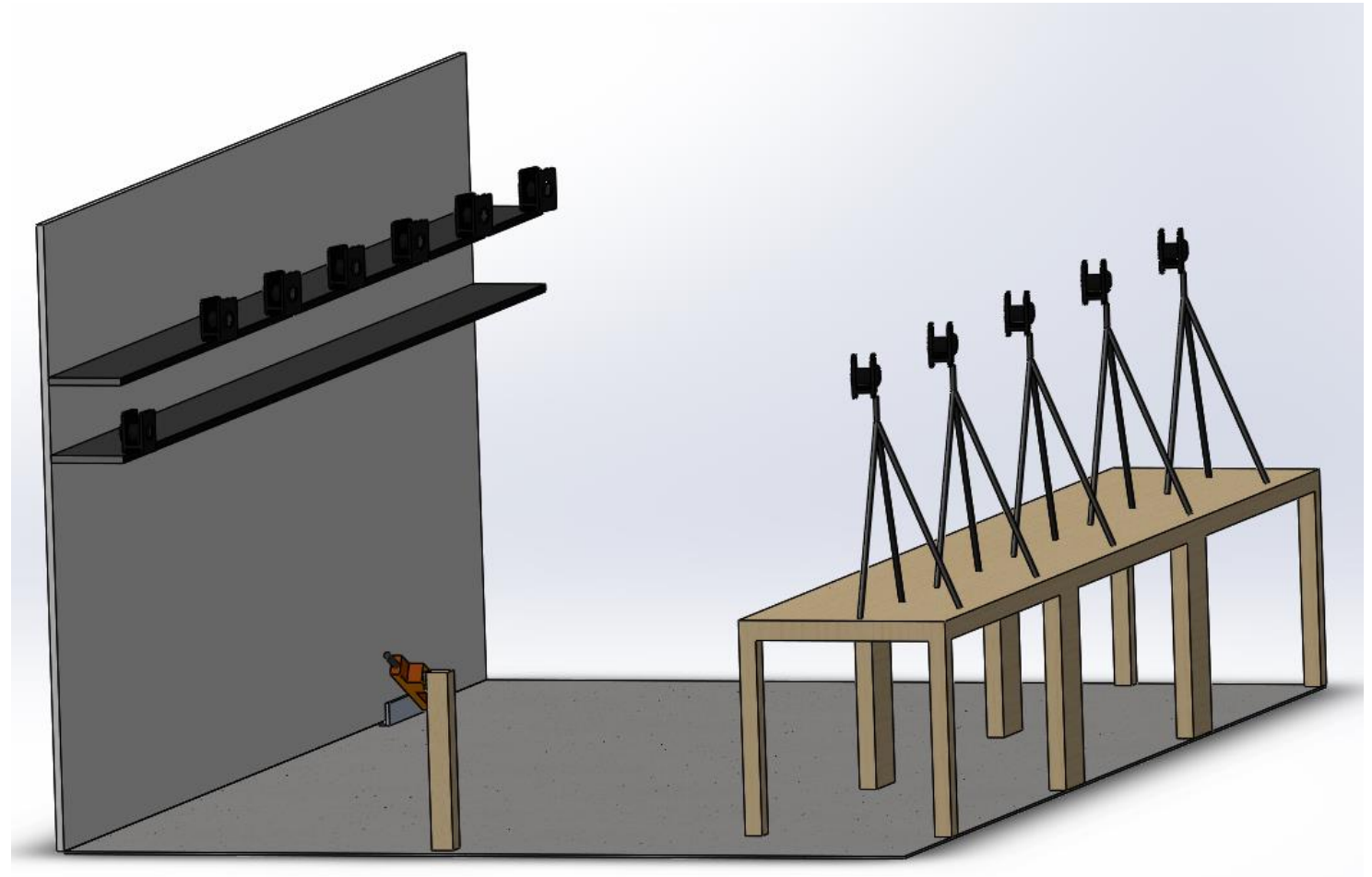

Figure 4.20: CAD model of Vicon test volume and camera configuration. 


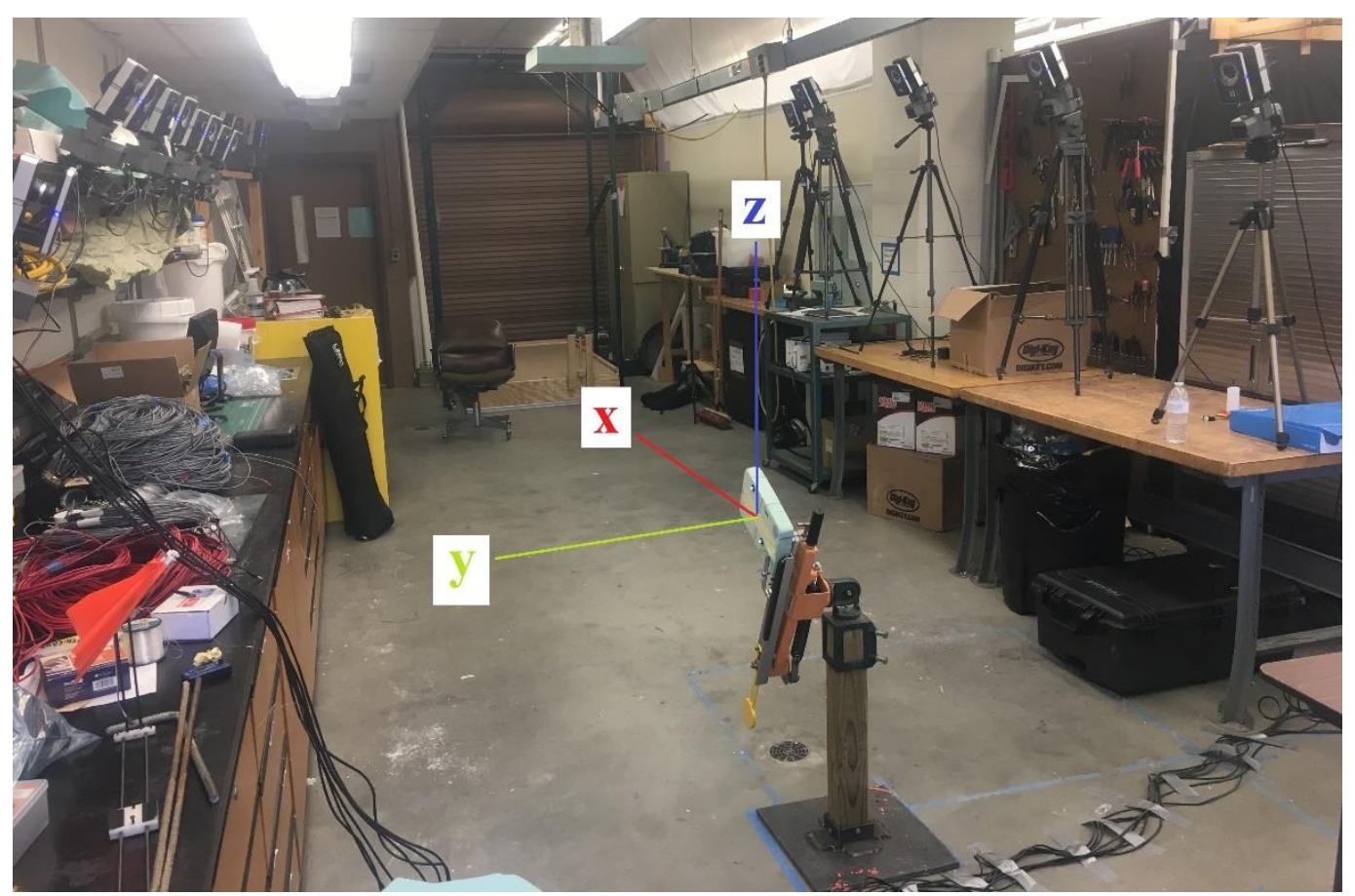

Figure 4.21: Vicon camera system experimental setup with coordinate system overlaid.

With reflective markers attached at points of interest, the blocks were launched through the volume. Before testing, all reflective material was removed from the volume. As the cameras emitted IR, noise in the system is created from other reflective material. To reduce the possibility of inaccurate tracking, a mask was used to remove reflective points within the volume that could not be physically removed (Figure 4.22). After the mask was created, the only reflective objects tracked by the system were the markers placed at the points of interest on the blocks.

Additionally, using the provided active wand, a camera calibration was performed to check the max world error and max image error of the system as well as allow the cameras to determine their orientation relative to each other. The active wand, shown in Figure 4.23, used preprogrammed distances between IR emitting points to calibrate the system. As the wand was waved through the volume, the cameras were able to calculate their location relative to each other, as well as determine the max world error and max image error. The Max world error as defined in the software documentation: "Displays the calibration error in millimeters. World error is calculated per camera from the Image Error in pixels and the distance of the camera to the center of the volume. Cameras further away, with the same image error, display a larger world error [10]." The Max image error is also defined in the software documentation: "This value (in RMS distance in camera pixels) indicates the accuracy of the $3 \mathrm{D}$ reconstruction of the markers. This value 
represents the difference between the $2 \mathrm{D}$ image of each marker on the camera sensor and the 3D reconstructions of those markers projected back to the camera's sensor. Acceptable values depend on factors such as camera type. The size of the capture volume, and the camera lens type [10]." For the purposes of testing with twelve cameras over a distance of $518 \mathrm{~cm}$, it was desired to keep both forms of error below 0.25. The max world error for 3000 refinement frames at a recording speed of $500 \mathrm{~Hz}$ was 0.23 , and the max image error was 0.15 .

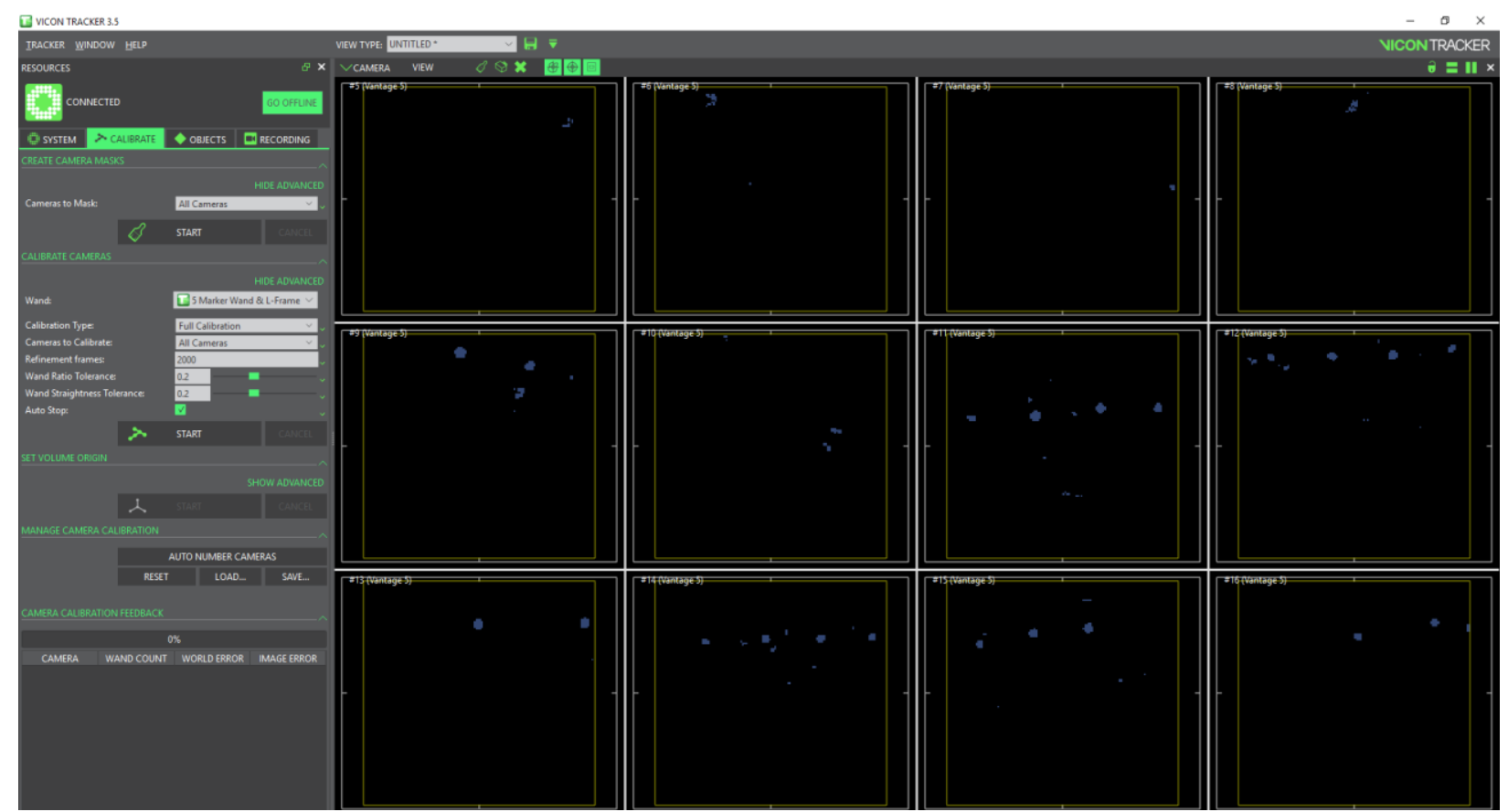

Figure 4.22: Vicon masking process to remove reflective material from the volume. Blue marks indicate reflective material such as other cameras, metal, etc. that has been masked.

Following the calibration process, the global origin was set using the active wand. Following the coordinate system of the active wand, the $x$-axis was aligned with the launcher with positive being downrange, positive z-axis up, and positive $y$-axis to the left, relative to the launching direction. The wand was first placed on the floor of the room to set the origin. However, the cameras were not able to capture the IR from the wand at such a low point. The wand was then placed upon a case to bring it into the frame of the cameras (Figure 4.24). 


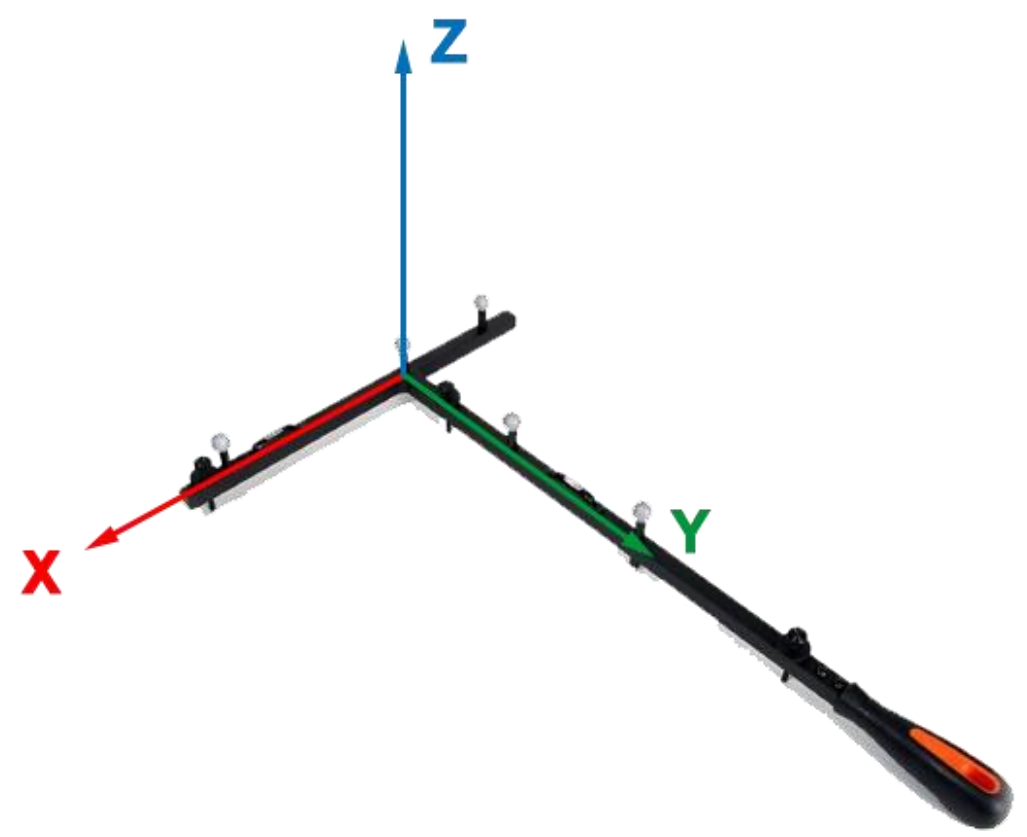

Figure 4.23: Vicon active wand, with coordinate system overlaid, used for system calibration.

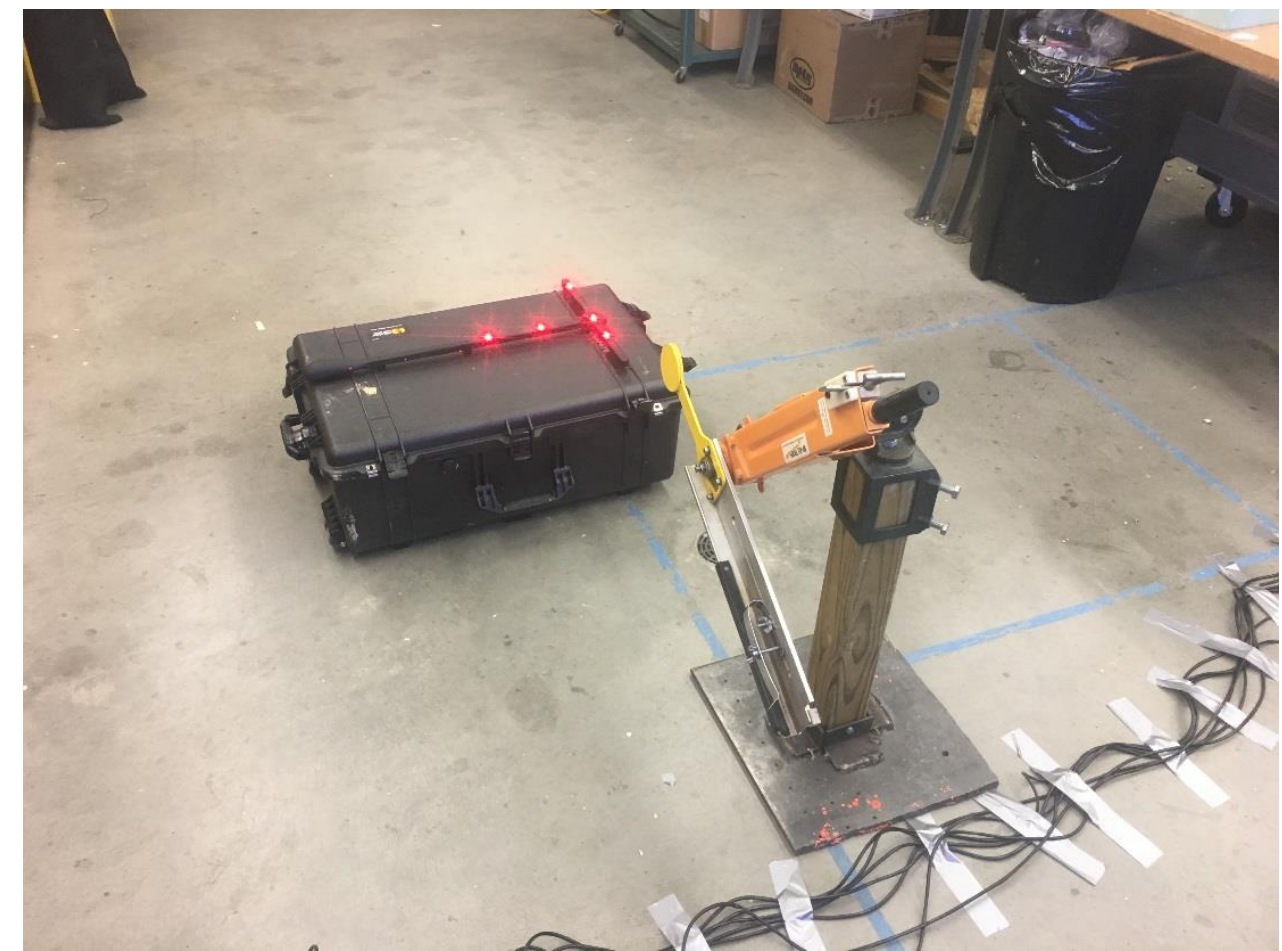

Figure 4.24: Setting the global origin for the Vicon system, using the active wand.

Finally, the active wand was removed and an object was created for each block size. Creating an object allows the Vicon system to identity the orientation of the block to calculate its position and orientation. It was selected that at least three cameras needed to have a marker in 
frame in order for it to register the point. Adjusting the lower limit of viewing cameras to two would have increased the probability that the system would have evaluated the body in a position that did not accurately represent the actual position of the body. For example, if the body was in an orientation similar to another orientation, the Vicon system could confuse the positions.

Once the object was created for the block size in question, it was placed in the launcher and the object's initial coordinate system was adjusted to coincide with the global coordinate system. The object coordinate system was placed on the center marker of the block. Then, the axes were aligned with the global axes. Finally, the coordinate system was moved to the center of the block by offsetting it on the $y$-axis to the midpoint between two points on opposing faces.

As in the tests with the launch and SA5, the blocks were placed in the launcher so that the locations for the additional masses were collinear with the edge of the holder (i.e. only half of the block's length was in contact with the holder) (Figure 4.25). To ensure that the blocks would travel through the volume at a height that could be captured by the cameras, the angle of the launcher was adjusted. The angles used during evaluation with the Vicon system, shown in Table 4-3, were the same as testing with the SA5. Using the same method to measure the throwing arm angle as with the SA5, the angle was determined using a protractor and level as shown in Figure 4.26. 


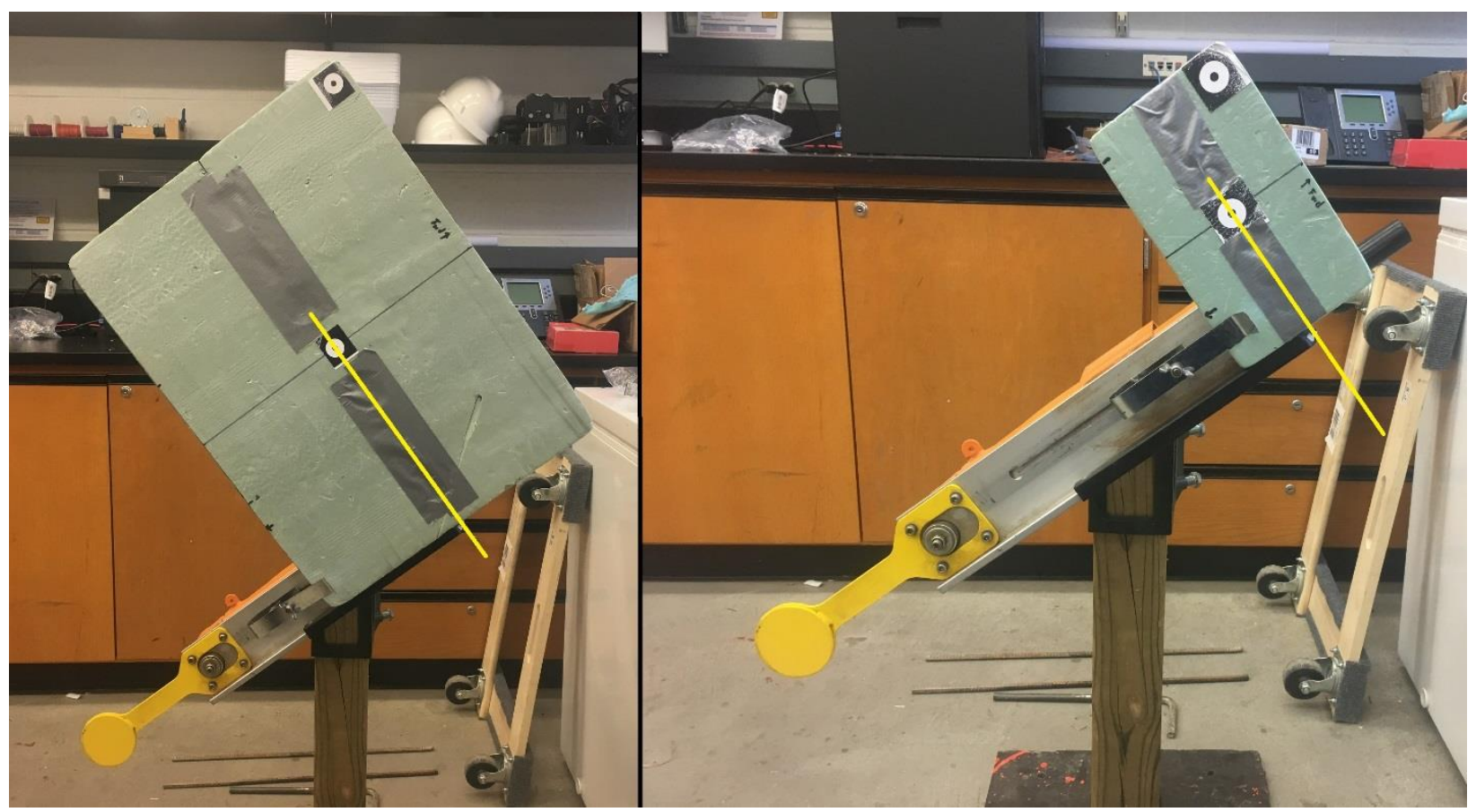

Figure 4.25: Comparison of block placement for Block 1 and Block 3 in the throwing mechanism. The blocks were placed so that only half of the L dimension was in contact with the mechanism. The yellow line indicates the centerline of the block as it extends through the tip of the holder.

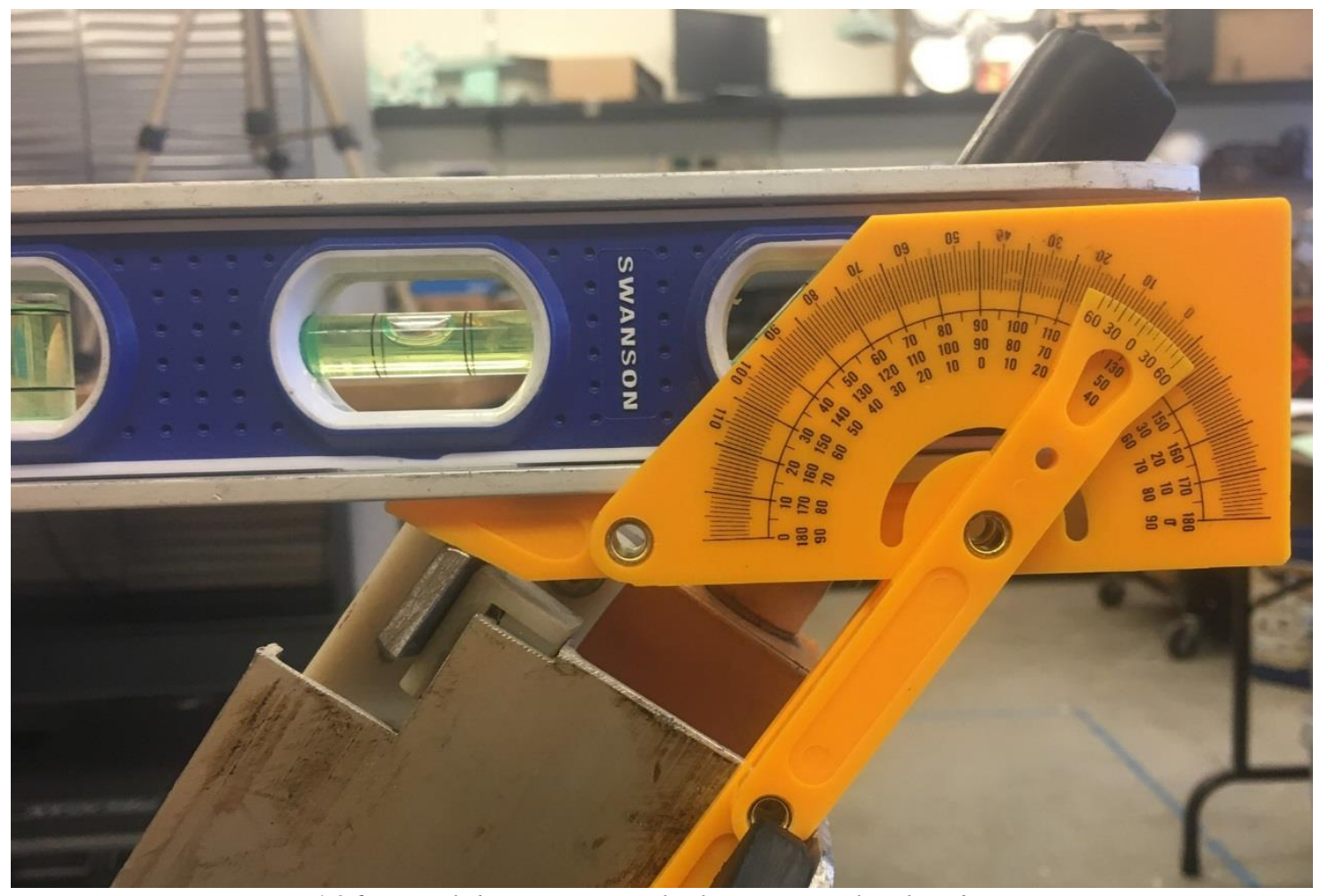

Figure 4.26: Initial thrower arm calculation using level and protractor. 
For each block size, three runs at each MMOI were conducted to reduce the probability of an inaccurate trajectory. Vicon calculates position of the body using quaternions. Therefore, when the data was exported as a .csv file, the quaternions needed to be converted to rates. This process is further explained in Chapter 5.5.

\section{Chapter 5 Motion Tracking Analysis}

The motion tracking software, Tracker, was used to analyze high speed video of each trajectory. Tracker uses contrast levels in the image to follow the same point within a selected window. Through use of a defined length scale and fps, Tracker calculates translational and angular velocity of the block as it moves across the table surface or falls vertically towards the ground. Average velocity and angular velocity were used to calculate dimensionless mass moment of inertia and dimensionless angular velocity. In excel, average values were calculated using the AVERAGE function, which follows the equation:

$$
\bar{x}=\frac{1}{N} \sum_{i=1}^{N} x_{i}
$$

Where $N$ is the number if samples and $x$ is the value of the sample.

The purpose of this experiment was to use dimensionless terms to relate MMOI and angular velocity. The common dimensionless terms used for analysis are shown in Table 5-1. To calculate these terms, other variables such as tangential velocity, density, angular velocity, MMOI and length were required. Variables such as length and density were measured prior to the experiments and averaged for all test articles. MMOI was calculated numerically using SOLIDWORKS due to the nonhomogeneous property of the material. Tracker or Vicon software was used to find the average linear velocity and angular velocity of the blocks. Each test method was evaluated using different length scale combinations and dimensionless terms to determine the existence of a scaling relationship. A comparison of data for each test is shown in the following sections. Additionally, a combination of all test data is also analyzed in section 5.6. 
Table 5-1: Dimensionless terms and equations.

\begin{tabular}{|c|c|c|}
\hline Dimensionless Term & Equation & Equation 5-1 \\
\hline Mass Moment of Inertia & $I^{*}=\frac{I}{\rho L^{5}}$ & Equation 5-2 \\
\hline Angular Velocity & $\omega^{*}=\frac{\omega L}{U}$ & Equation 5-3 \\
\hline Froude Number & $F r=\frac{U^{2}}{L g}$ & Equation 5-4 \\
\hline Rotational Reynolds Number & $R e_{\text {rot }}=\frac{L^{2} \omega}{v}$ & Equation 5-5 \\
\hline Reynolds Number & $R e=\frac{U L}{v}$ & Equation 5-6 \\
\hline Energy Ratio & $\frac{E_{\text {rot }}}{E_{\text {total }}}=\frac{I \omega^{2}}{\left(I \omega^{2}+m U^{2}\right)}$ & \\
\hline
\end{tabular}

The importance of choosing the correct length scale cannot be overlooked. Therefore, numerous length scales were calculated to find the most appropriate scaling analysis. Six options were examined: hydraulic diameter, length to the bolt, face surface area divided by length, largest block dimension, volume divided by total surface area, and the product of aspect ratio and length to the bolt. For the four sizes of blocks used, each length scale can be seen in Table 5-2, along with its corresponding equation. 
Table 5-2: Length scales analyzed to calculate dimensionless terms.

\begin{tabular}{|c|c|c|c|c|c|c|}
\hline $\begin{array}{l}\text { Length } \\
\text { Scale }\end{array}$ & $\begin{array}{c}\text { (Aspect Ratio) } \\
\mathrm{x} \\
\text { (Length to } \\
\text { Bolt) } \\
{[\mathrm{cm}]} \\
\end{array}$ & $\begin{array}{l}\text { Hydraulic } \\
\text { Diameter } \\
{[\mathrm{cm}]}\end{array}$ & 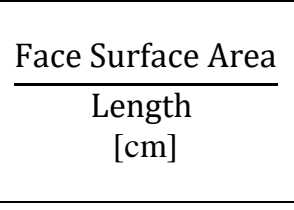 & $\begin{array}{l}\text { Length } \\
\text { to Bolt } \\
{[\mathrm{cm}]}\end{array}$ & $\begin{array}{l}\text { Largest } \\
\text { Dimension } \\
{[\mathrm{cm}]}\end{array}$ & $\frac{\text { Volume }}{\text { Total Surface Area }}$ \\
\hline Equation & $\frac{W}{L}(0.125 W)$ & $\frac{4(W L)}{2(W+L)}$ & $\frac{H W}{L}$ & $0.125 \mathrm{~W}$ & W & $\frac{L W H}{2(L W+H W+L H)}$ \\
\hline \multicolumn{7}{|c|}{ Block 1} \\
\hline MMOI 1 & 5.31 & 19.05 & 6.35 & 3.18 & 25.4 & 0.54 \\
\hline MMOI 2 & 10.60 & 19.05 & 6.35 & 6.35 & 25.4 & 0.54 \\
\hline MMOI 3 & 15.91 & 19.05 & 6.35 & 9.53 & 25.4 & 0.54 \\
\hline \multicolumn{7}{|c|}{ Block 2} \\
\hline MMOI 1 & 10.60 & 38.10 & 8.47 & 6.35 & 50.8 & 0.79 \\
\hline MMOI 2 & 21.21 & 38.10 & 8.47 & 12.70 & 50.8 & 0.79 \\
\hline MMOI 3 & 31.81 & 38.10 & 8.47 & 19.05 & 50.8 & 0.79 \\
\hline \multicolumn{7}{|c|}{ Block 3} \\
\hline MMOI 1 & 10.13 & 52.25 & 6.77 & 7.62 & 61.0 & 0.84 \\
\hline MMOI 2 & 20.27 & 52.25 & 6.77 & 15.24 & 61.0 & 0.84 \\
\hline MMOI 3 & 30.40 & 52.25 & 6.77 & 22.86 & 61.0 & 0.84 \\
\hline \multicolumn{7}{|c|}{ Block 4} \\
\hline MMOI 1 & 12.05 & 21.73 & 12.73 & 4.8 & 38.1 & 0.68 \\
\hline MMOI 2 & 23.85 & 21.73 & 12.73 & 9.5 & 38.1 & 0.68 \\
\hline MMOI 3 & 35.90 & 21.73 & 12.73 & 14.3 & 38.1 & 0.68 \\
\hline
\end{tabular}

\subsection{SA5 High Speed Tracking}

Although an $18 \mathrm{~mm}$ lens has relatively low distortion, a video calibration was performed to increase accuracy. A comparison of an original image and a calibrated image is shown in Figure 5.4. Note that higher distortion can be seen at the edges of the images. Once the camera was in place, a $3 \mathrm{ft}$. $x 4 \mathrm{ft}$. grid containing 4 in. $\mathrm{x} 4$ in. black and white squares was held in view of the camera (Figure 5.1). This grid was angled approximately 15 degrees in each direction, creating four distinct planes. The process of angling the grid was repeated in three places within the video to account for the field of view of the camera. 


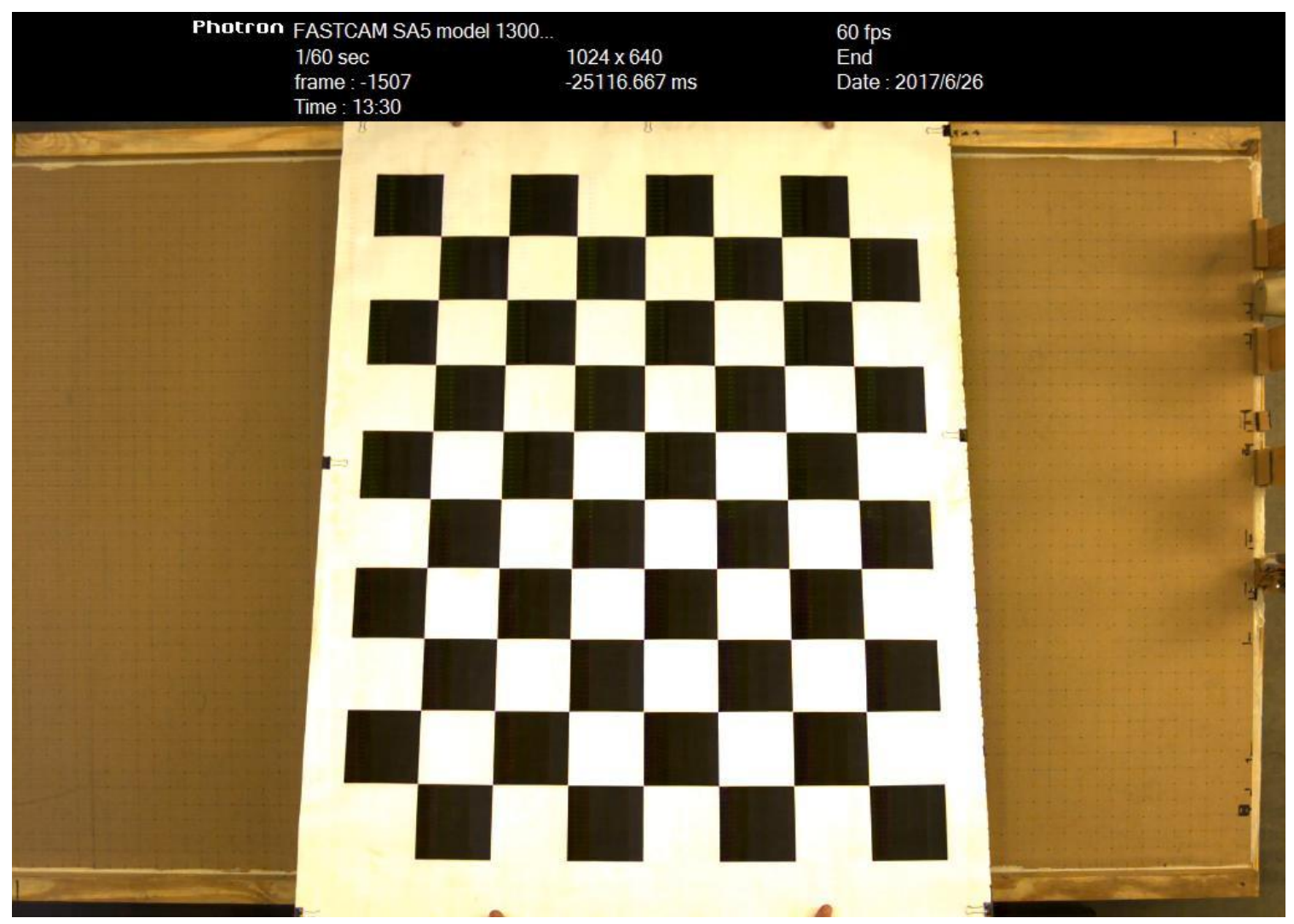

Figure 5.1: Grid of 4 inch squares used to calibrate images to reduce edge distortion.

Images at each location and angle from the calibration video were extracted and used for calibration. A total of 10-20 images were used to generate a MATLAB script to correct the videos. Using the Graphical User Interface (GUI) option Camera Calibrator in MATLAB (Appendix A), the images from the calibration video were used to generate a mean pixel error (Figure 5.2). A low mean pixel error was desired because it generated little or no distortion. Howeer, some images may cause large changes to the average mean pixel error. Images that deviated far from the mean were deleted and the mean pixel error was recalculated. Once the mean pixel error was below a value of 1, the image was digitally constructed to recreate the calibration as a figure (Figure 5.3). Then, a code was generated and the parameters for calibration were exported as the variable CameraParams. A mean pixel error of less than 1 was selected to assure the images would process with accurate scaling. These parameters can only be applied to images. To calibrate videos of block tests, the videos were uploaded into Adobe Photoshop CC 2015 and rendered to images. The images were run through a code in MATLAB to apply the variable CameraParams to each image, then combine the calibrated images into an $A V I$ file. Finally, the calibrated video was loaded into Tracker for analysis. 


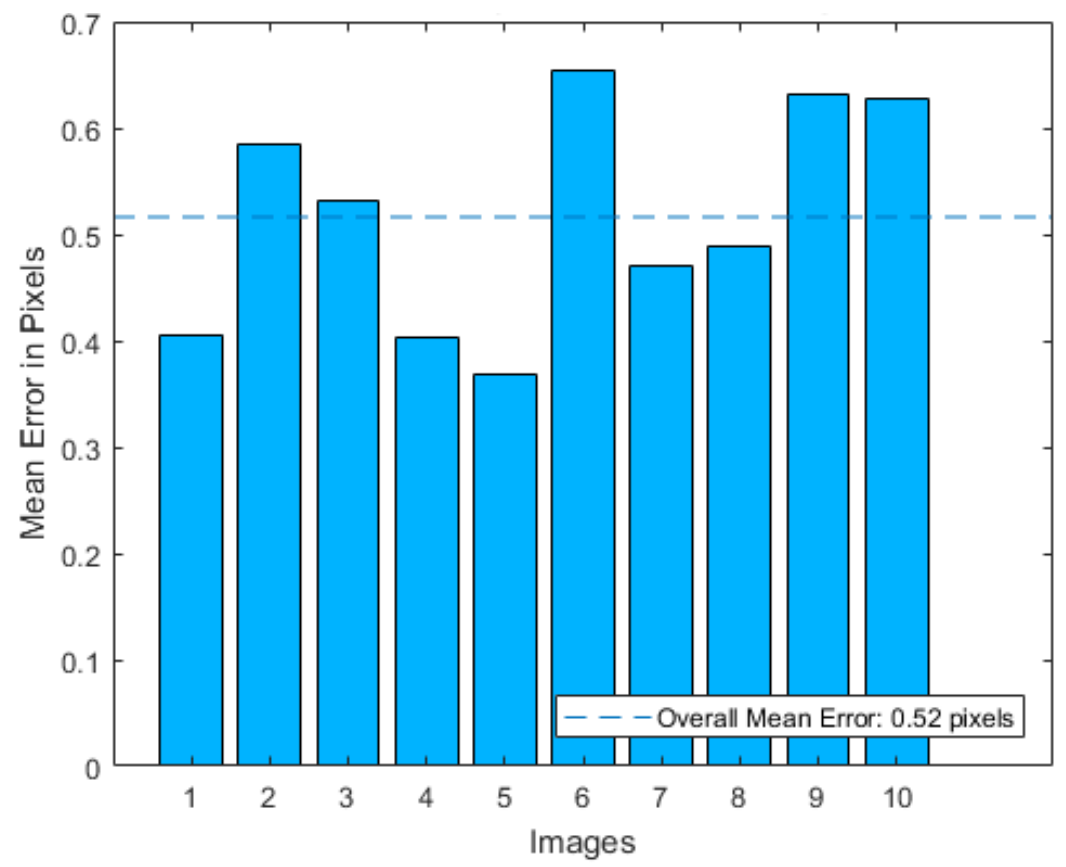

Figure 5.2: Mean reprojection error per image calculated by MATLAB Camera Calibration App. An overall mean error of less than 1 pixel was used to calibrate images.

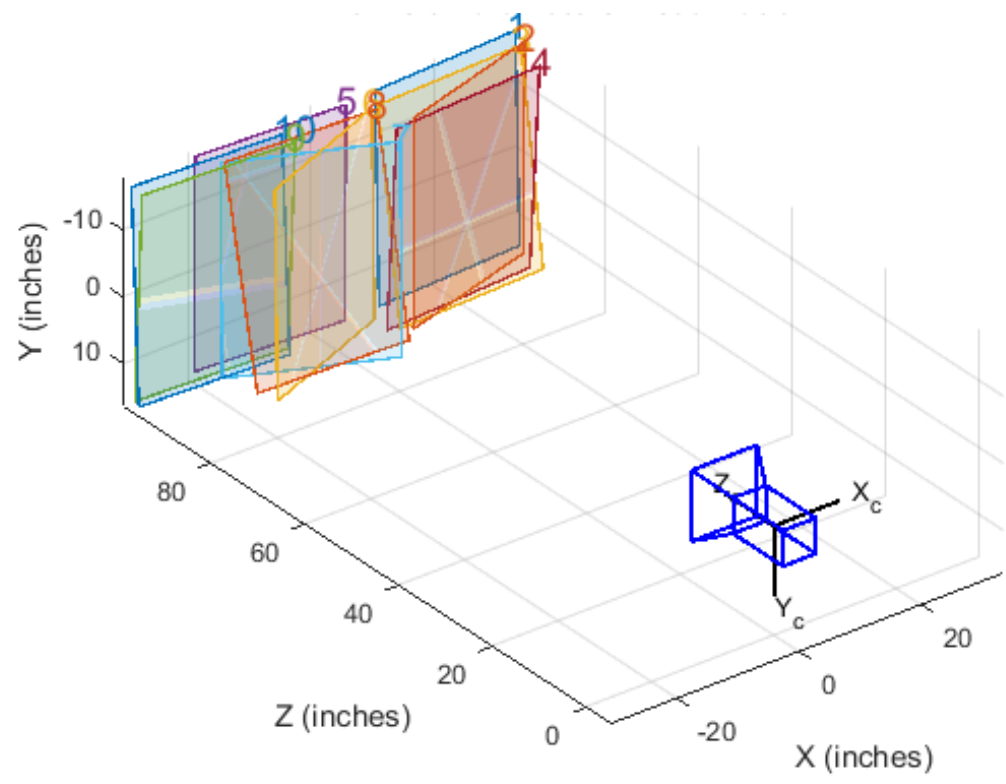

Figure 5.3: MATLAB extrinsic projection of camera location in relation to calibration board images. 

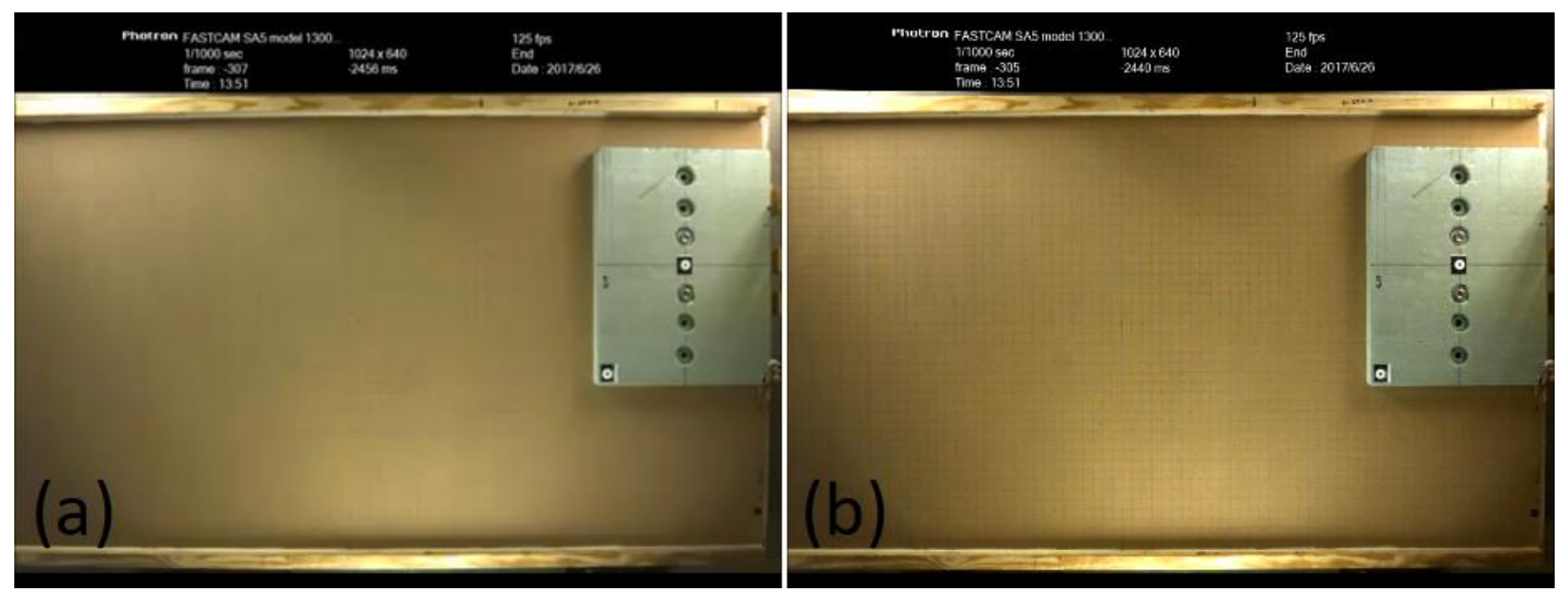

Figure 5.4: Comparison of original image, (a), and calibrated image, (b).

Error in the tracking software was tested by determining a stationary point in space (Figure 5.5). That is, the block was placed on a stool and the contrast sticker was selected to be tracked. Since the block was not moving, the software should output the same coordinates for each timestep. Error in the tracking software caused less accurate calculations for position and velocity. The error was quantified in inches to evaluate a range of distances over which the point may drift. Two sets of data were recorded, datal and data2, for each coordinate. The data collected for each trial was recorded over the same amount of time steps, as well as selected pixels for tracking. When the data from each run was plotted, each position overlapped with previous data. As shown in Figure 5.6, the two sets of data in each plot overlap as if only one set of data is being evaluated. The figure also shows that, for the x-location, the range of position in which the point was tracked is between 0.0042 inches and 0.00445 inches. Taking the difference of the maximum and minimum values determined by Tracker gives the total possible deviation of 0.00025 inches in the $\mathrm{x}$-axis. Similarly, the $\mathrm{y}$-axis has a maximum of 0.00035 inches. Relative to the resolution of the block measurement system, manually selected pixels for tracking, and other sources of error; the error produced by tracking between points was deemed negligible. 


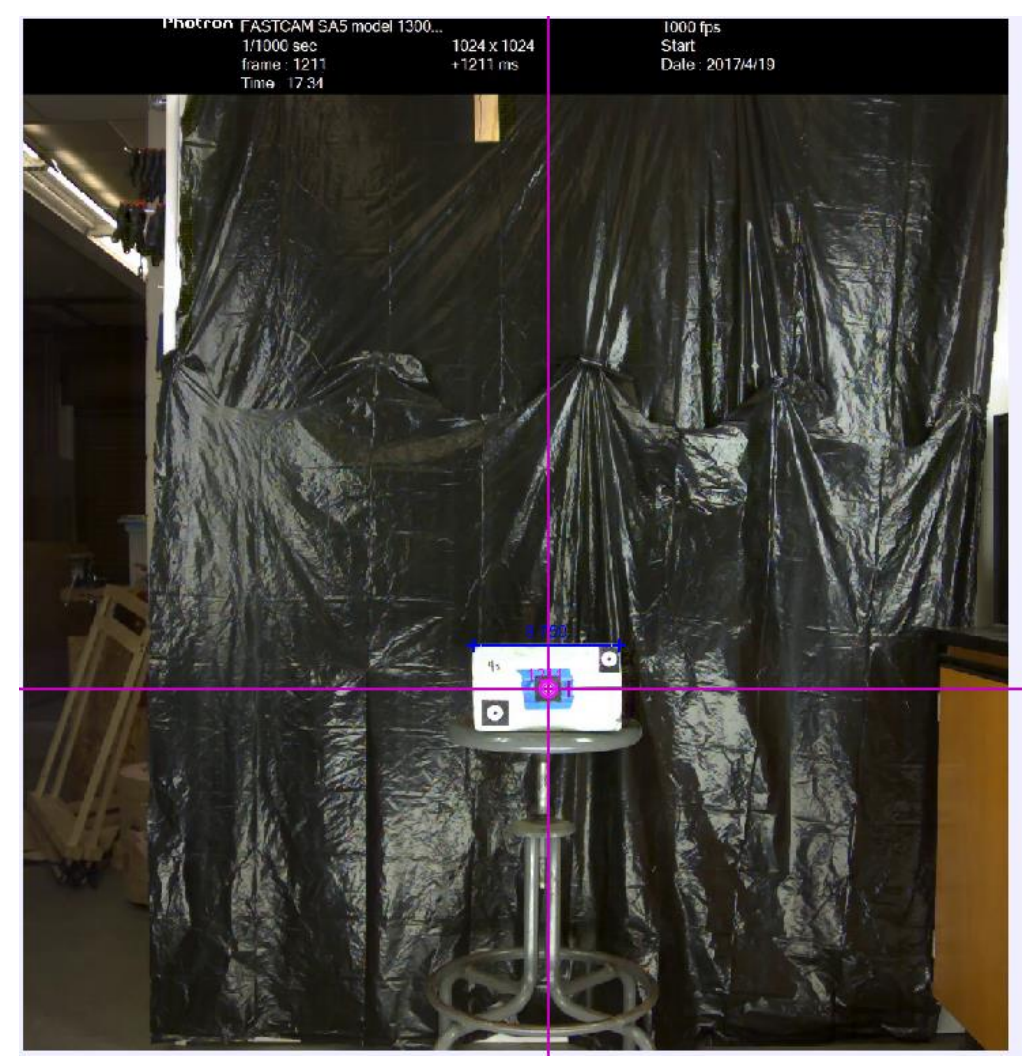

Figure 5.5: Tracking a stationary point to determine noise in the software.
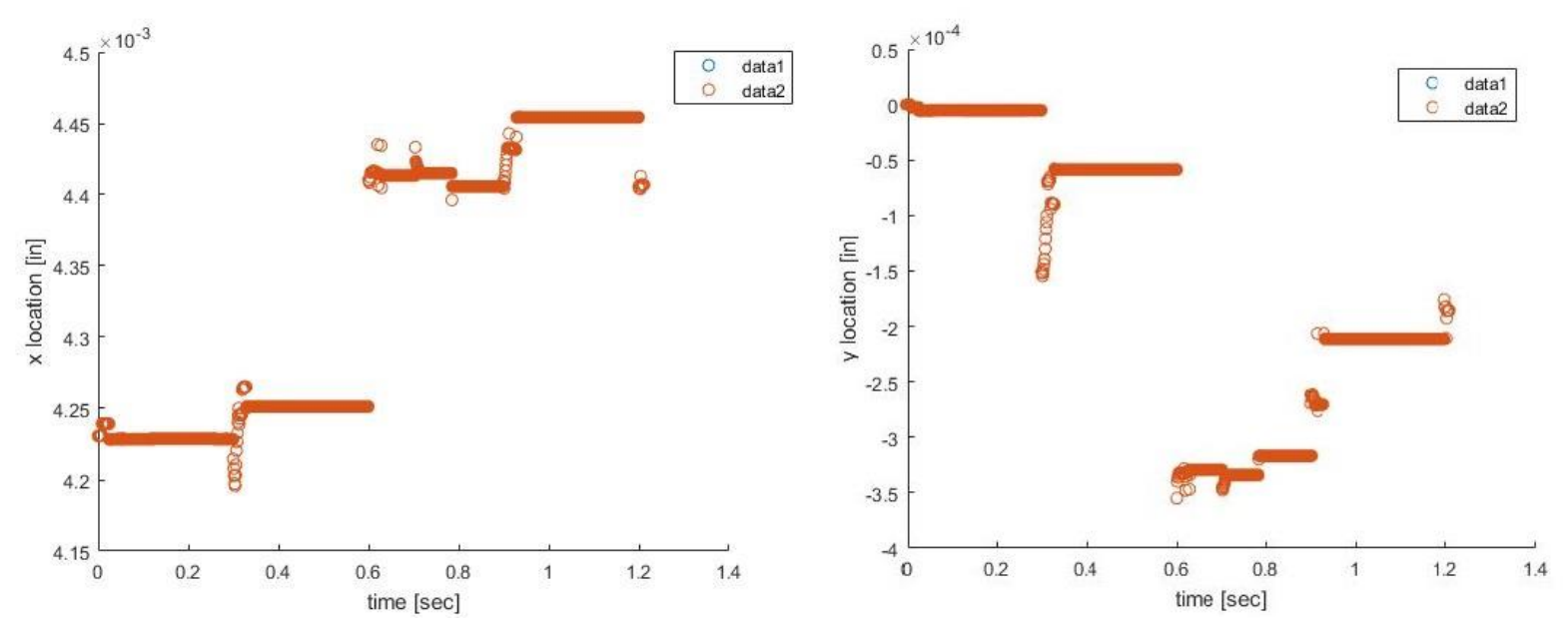

Figure 5.6: Stationary point position data for $x$ and $y$ positions.

To determine the number of tests required for accurate calculations, data was collected over five runs. The velocity of the marker on the block was averaged for each time step of each run until the difference between the average and the next run was less than $5 \mathrm{~cm} / \mathrm{sec}$. It was found that three runs provided an accurate representation of block velocity. That is, the average velocity 
throughout the trajectory did not change significantly after three runs. It is important to note that error accumulated as time increased. For example, the initial position of the block was always within a few millimeters, limited by the accuracy of user defined pixel selection. As the block rotated through its trajectory, other factors such as small inconsistencies with mounting location in the drop mechanism, weight bias towards one surface from the additional weights, and friction between the block and horizontal plane cause greater inaccuracies in data. As shown in Figure 5.7, the position of Block 1 through time during the horizontal test has a greater range at the end of its trajectory. Figure 5.7 indicates the range over which the block can be located for six runs.

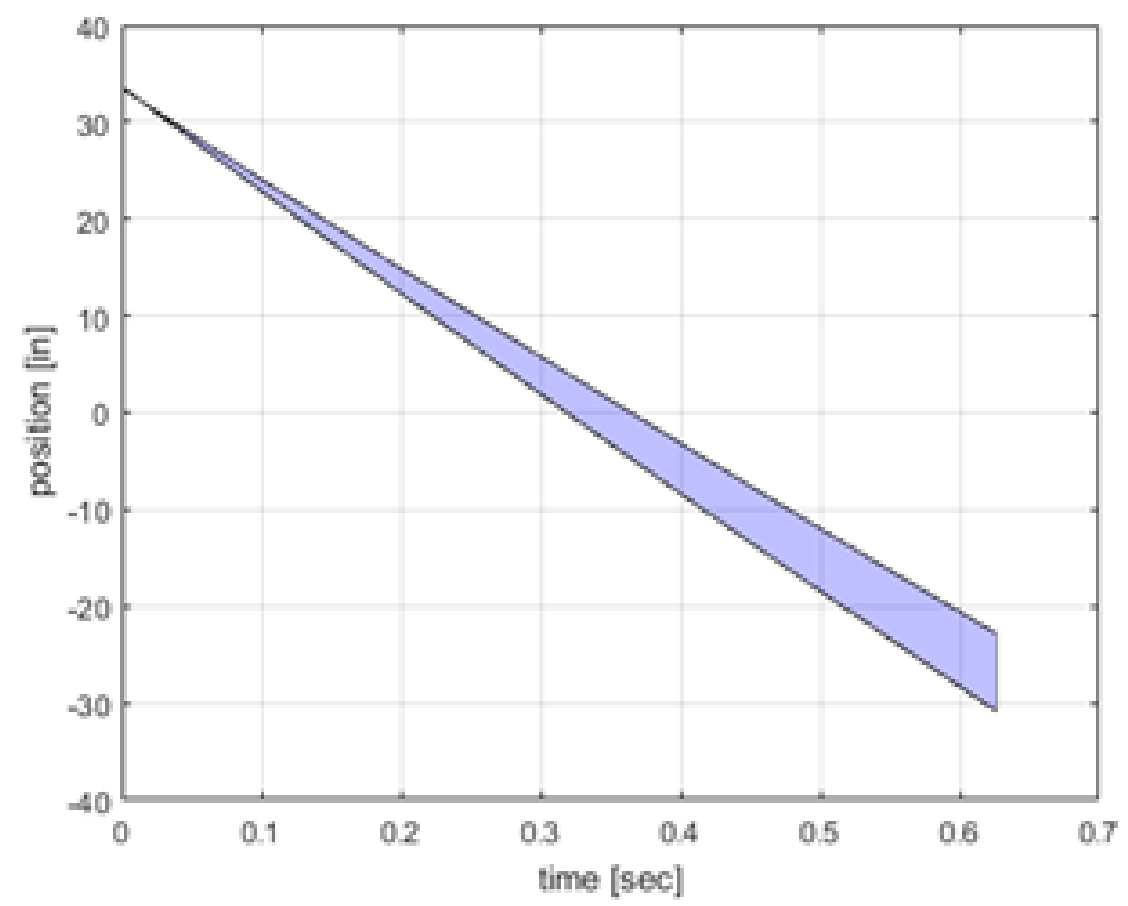

Figure 5.7: Range of position for Block 1 - MMOI 1.

An additional method used to determine the accuracy of the testing was to plot data for dimensionless angular velocity versus time (Figure 5.8). The data showed a similar trend for each set of data. As expected, the data did not fall on the same line due to the changing MMOI value. This was just to determine the trend followed by the data. It is important to note that for Block 4 the range over which $\omega^{*}$ was calculated increased, relative to the other block sizes. From this, it was determined that Block 4 required the most attention when testing to attain accurate data. 


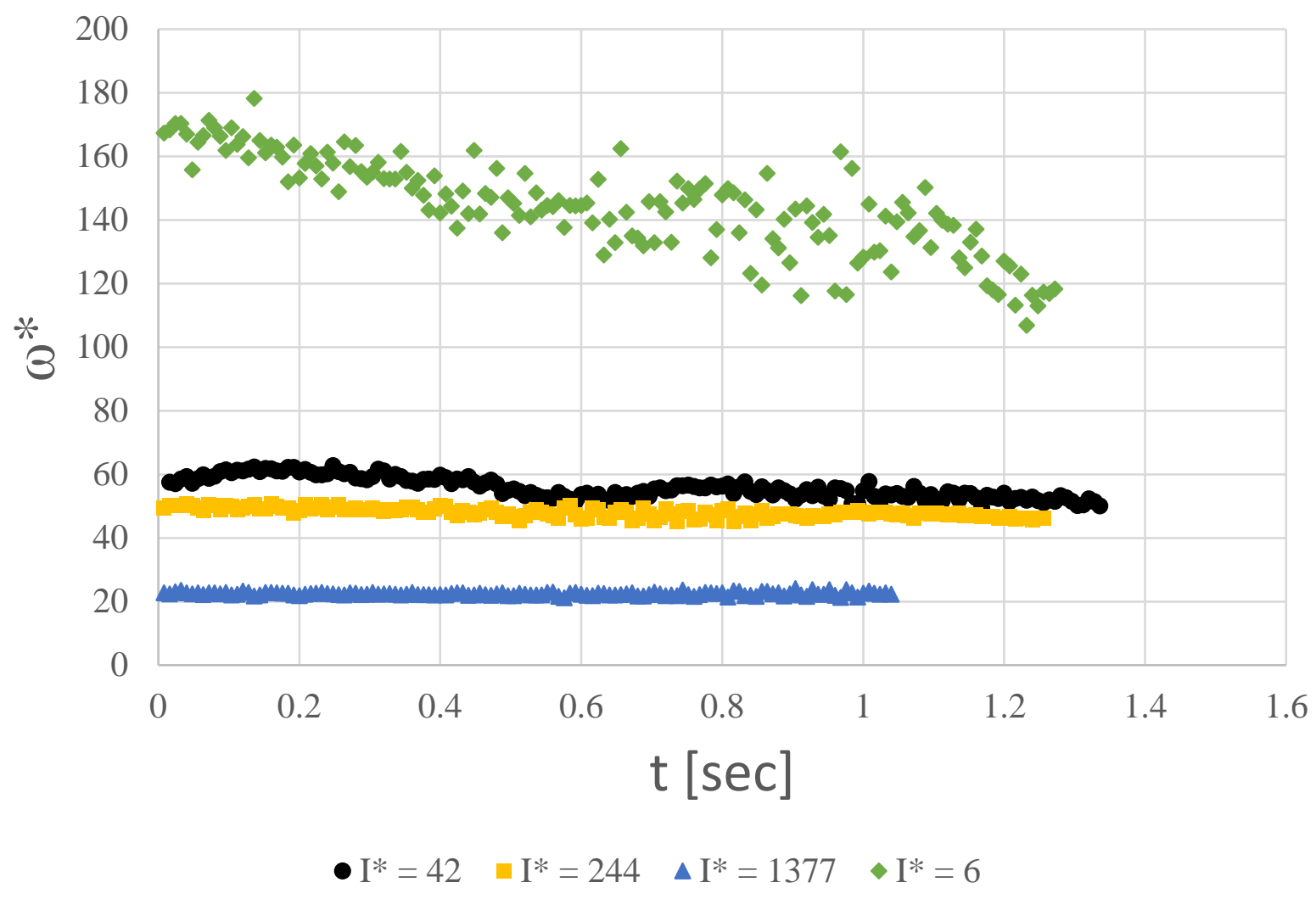

Figure 5.8: Data trends for changing MMOI

\subsection{D Horizontal Plane}

To begin the testing process for the foam blocks on the air table, after calibration, a suitable camera fps between 500 and 1000 was selected and aperture adjusted to allow for clear video. The purpose of initial testing was to determine Tracker's capabilities and optimize accuracy of tracking points. Using high contrast points of black and white circles was the easiest combination for the software to track. For translational data, a point was placed in the center of the block and tracked with respect to a user defined coordinate system. For angular data, a point was placed in the corner of the block and tracked with respect to the center point. The software, Tracker, was used to analyze position and velocity data from the high-speed video. Position goes from positive to negative due to the default coordinate system of the software (Figure 5.9). A more suitable coordinate system is implemented later in the study. 


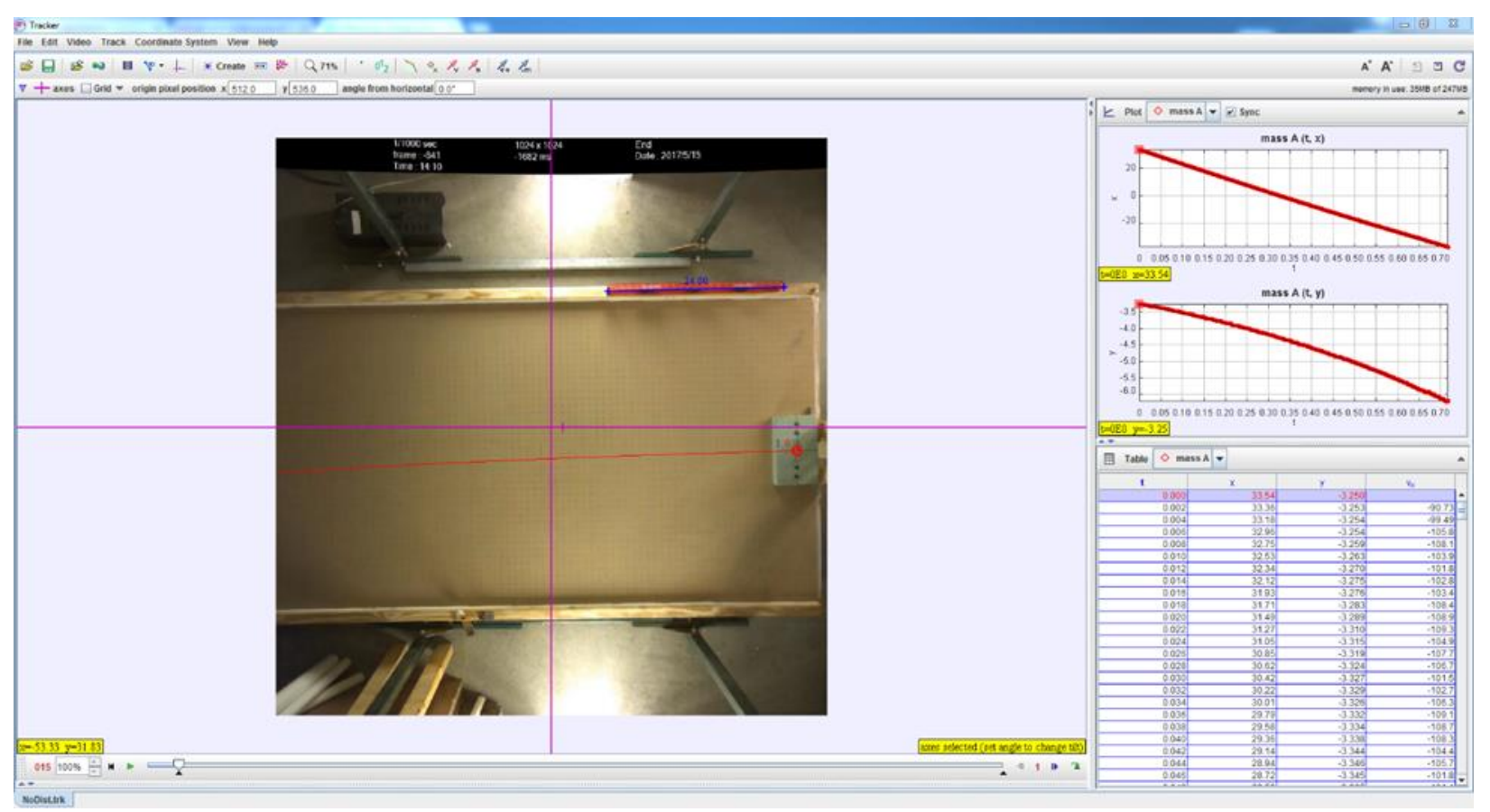

Figure 5.9: Default Tracker coordinate system (blue) and trail of tracked velocity (red).

Three runs for each MMOI of each block were performed in a random order to decrease any discrepancies from small changes in the system throughout testing. In analyzing the capabilities of Tracker, only translational data of the block was tested. However, purely translational motion was difficult to obtain. The block was struck by the firing mechanism as close to its center of mass as possible. By maintaining a constant initial force on the block and omitting torque, the velocity of each individual block should be the same for each of its MMOI, since moving the additional weights to change the MMOI did not affect the total mass of the block. Maximum and minimum values of velocity over time were determined and plotted as a range in which the block may travel. The ranges shown in Figure 5.10 are affected by initial conditions, friction between the block and table, drag on the block, and points manually selected in Tracker. As the time of translation increases, a larger range of displacement of the minimum and maximum position increases. However, over a distance of approximately six feet, the maximum range in which the point on the block was tracked was within eight inches. In ideal conditions, no range would exist and each run would produce exactly the same trajectory. For a truly frictionless surface with no drag on the block, the velocity should remain constant over time, providing a horizontal line when plotted. However, drag force on the block, as well as friction between the block surface and table, caused a decrease in velocity over time. This can be seen in Figure 5.10 as the velocity starts to decrease more dramatically after approximately 0.4 seconds. 
For Figure 5.10, it was attempted to induce zero torque on the block with the firing mechanism. Therefore, a change in the MMOI of the block should not affect its velocity. Theoretically, only one line should exist if all conditions were exactly the same. Each run, regardless of MMOI, should produce the same velocity because the mass did not change. However, initial conditions cannot be replicated completely. The drag and friction cause a slight decrease in velocity over time. Also, even though the test was meant to have zero rotation, a slight torque will always be present, causing some of the energy to be converted to rotational motion. The increase in velocity from one MMOI to the other for these tests was assumed to be due to a longer impulse from the hammer. This significant difference in translational velocity only occurred in the Block 1, though. For Block 2 and Block 3, a consistent and expected velocity profile was measured and is shown in Figure 5.10.
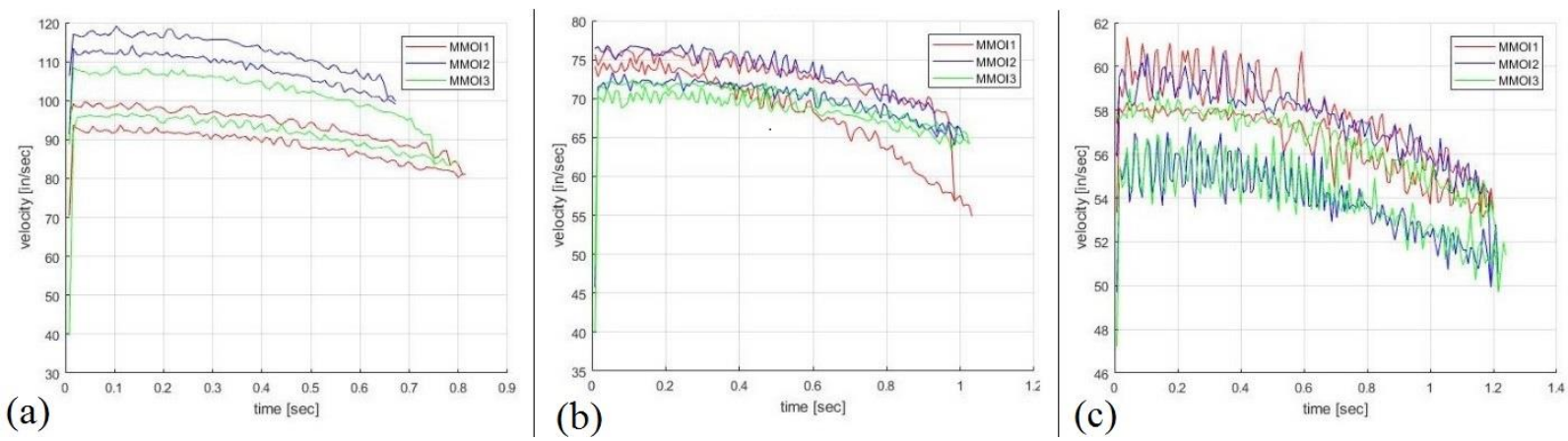

Figure 5.10: (a) Range of velocity variation over three runs for Block 1. (b) Range of velocity variation over three runs for Block 2. (c) Range of velocity variation over three runs for Block 3.

Noise in the data is due to Tracker software calculations. Velocities were calculated numerically by the equation:

$$
V=\frac{x_{2}-x_{1}}{t_{2}-t_{1}}
$$

Because the tracker software was "searching" for a preset pattern of color levels, each point contained a small amount of error. For example, if the change in velocity over one time step is 94 in/sec to $81 \mathrm{in} / \mathrm{sec}$, an associated distance must be causing the discrepancy since the time step between data points remains constant. A sudden decrease in velocity of $13 \mathrm{in} / \mathrm{sec}$ is unlikely. Even more unlikely is the increase of velocity from one time step to the next. With a change of 13 $\mathrm{in} / \mathrm{sec}$, based on the position data, it is assumed that the program noted a position that was slightly off from actual location. For the program to calculate a velocity of $94 \mathrm{in} / \mathrm{sec}$, the $\Delta \mathrm{x}$ must be 0.188 
inches for a time step of 0.002 seconds. Similarly, a velocity of $81 \mathrm{in} / \mathrm{sec}$ has a $\Delta \mathrm{x}$ of 0.162 inches. If Tracker is unable to find the point due to inadequate lighting or sensitive settings, the user must redefine an area in which to match. With a video resolution of 20 pixels/inch, redefining a position that is only 4 pixels off from the previous point can produce an inaccuracy of 0.25 inches in $\Delta x$. Although new user-defined points carried the most error, points selected by the software also carry a similar error, producing noise in the data.

Theoretical values were calculated using the assumption of a perfectly elastic collision. In which, kinetic energy is conserved. Conservation of momentum was used to determine the final translational velocity of both the hammer and the block, as well as the final angular velocity of the block. The following derivations use specific variables to indicate initial and final locations, as well as subscripts to indicate the block, hammer, or stage. For example, subscript $h$ implies a property of the hammer, subscript $b$ implies a property of the block, and $u$ and $v$ indicate velocity before and after impact, respectively. The problem was broken down into two stages (Figure 5.11). The first stage was defined as the time the hammer was dropped from a height, $h_{l}$, to just before it impacted the block. This stage was used to calculate the tangential velocity of the hammer, $u_{h}$, as it impacted the block, through use of conservation of energy. Once the impact velocity was known, stage two was used to determine the final tangential velocity of both the hammer and the block, as well as the angular velocity of the block. Assuming the head of the hammer contained enough of the total mass that it could be treated as a weight attached to a massless handle, conservation of energy was simplified to:

$$
m_{h} g h_{1}=\frac{1}{2} m_{h} u_{h}^{2}
$$

If the handle was not assumed to be massless, the moment of inertia of the hammer must be accounted for in the conservation of energy equation. The height at which the hammer falls was determined by the relationship between drop angle and the radius of the hammer, $R$.

$$
\begin{array}{ll}
h_{1}=R-R \cos \theta & \theta \leq 90^{\circ} \\
h_{1}=R+R \sin \left(\theta-90^{\circ}\right) & \theta>90^{\circ}
\end{array}
$$


For the case of a $90^{\circ}$ drop, $h_{l}$ is equal to the length of the hammer. Therefore, solving for $u_{h}$ yielded an impact velocity of $234 \mathrm{~cm} / \mathrm{sec}$.

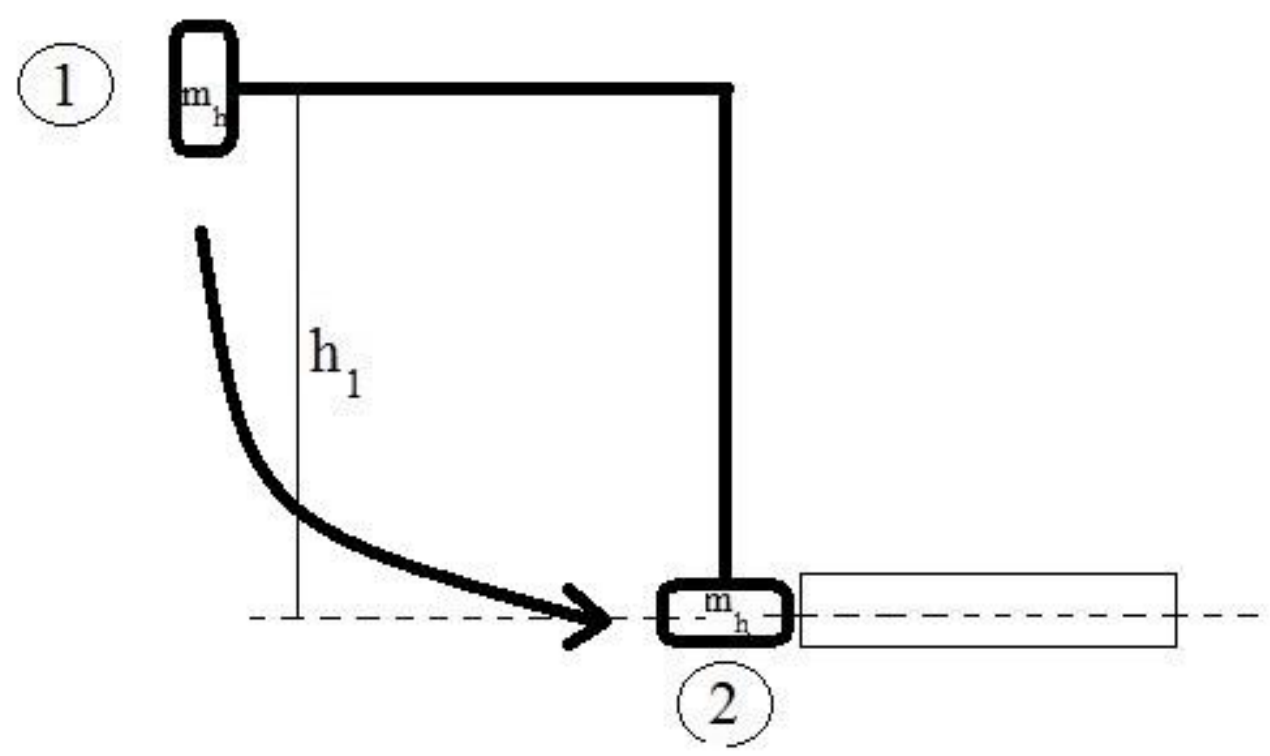

Figure 5.11: Calculating hammer impact velocity using conservation of energy in two stages. Stage 1 indicates initial height of the hammer. Stage 2 indicates the position of the hammer just before contact with the block.

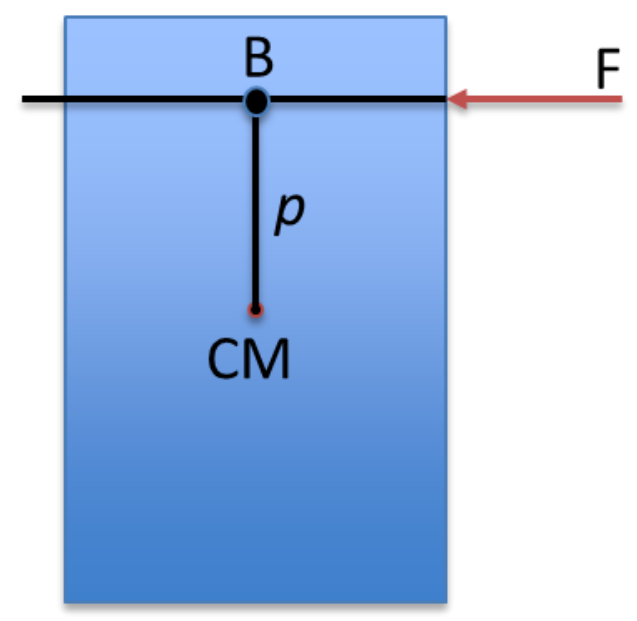

Figure 5.12: Impact location on the block during contact from hammer.

Once initial velocity was calculated, stage two of the motion was determined by combining conservation of momentum, conservation of angular momentum and conservation of energy. Starting with the general form of conservation of momentum and the free body diagram shown in Figure 5.12, the derivation for final velocities is as follows.

$$
m_{h} u_{h}+m_{b} u_{b}=m_{h} v_{h}+m_{b} v_{b}
$$


For this case, the initial velocity of the block, $u_{b}$, was 0, reducing Equation 5-7 to Equation 5-8.

$$
m_{h} u_{h}=m_{h} v_{h}+m_{b} v_{b} \quad \text { Equation 5-8 }
$$

Next, the angular momentum of the block was accounted for. The angular momentum was caused by the torque exerted on the block by the hammer impact at a distance, $P$, from the center of mass. The general angular momentum was determined by the relationship,

$$
L=\vec{r} \times \vec{p}
$$

Equation 5-9

where $p$ is simply the linear momentum, $m v$, and $r$ is the relative distance of the hammer to the center of mass of the block. Although $r$ is a relative distance, the cross product in Equation 5-9 was reduced to Equation 5-10.

$$
L=m u(P \sin \theta)
$$

Equation 5-10

From Equation 5-10, the conservation of angular momentum was calculated using angular momentum of the hammer before and after impact as well as angular velocity of the block after impact. To keep units consistent, the angular velocity was multiplied with the MMOI of the block.

$$
m_{h} u_{h} P=I_{b} \omega_{b}+m_{h} v_{h} P
$$

Finally, since it was assumed that the impact was perfectly elastic, conservation of energy was used to derive the final relationship.

$$
\frac{1}{2} m_{h} u_{h}^{2}=\frac{1}{2} m_{b} v_{b}^{2}+\frac{1}{2} m_{h} v_{h}^{2}+\frac{1}{2} I_{b} \omega_{b}^{2}
$$

Equation 5-12

Equation 5-8, Equation 5-11, and Equation 5-12 were then used to determine the three unknowns in the system: $v_{h}, v_{b}$, and $\omega_{b}$. Solving Equation 5-8 for $v_{b}$ and Equation 5-11 for $\omega_{b}$, each was plugged into Equation 5-12 to get a relationship only dependent on $v_{h}$. Combining the equations yields Equation 5-13.

$$
m_{h} u_{h}^{2}=m_{b}\left[\frac{m_{h}}{m_{b}}\left(u_{h}-v_{h}\right)\right]^{2}+m_{h} v_{h}^{2}+I_{b}\left[\frac{m_{h} P}{I_{b}}\left(u_{h}-v_{h}\right)\right]^{2}
$$

Solving Equation 5-13 for $v_{h}$ gives an independent equation for the hammer velocity after impact. $v_{h}$ was used to back-calculate linear velocity of the block after impact and angular velocity of the block. For a simple check of calculations, the final height of the hammer was also calculated. If 
the final height reached by the hammer was greater than its initial height, the system would be gaining energy somewhere, indicating a problem with the equations. The final independent equations for comparing experimental data were simplified to:

$$
\begin{gathered}
v_{h}=\frac{m_{h} u_{h}\left(\frac{1}{m_{b}}+\frac{P^{2}}{I_{b}}\right)-u_{h}}{\left(\frac{m_{h}}{m_{b}}+\frac{m_{h} P^{2}}{I_{b}}+1\right)} \\
v_{b}=\frac{m_{h} u_{h}}{m_{b}}\left[1-\frac{m_{h}\left(\frac{1}{m_{b}}+\frac{R^{2}}{I_{b}}\right)-1}{\left(\frac{m_{h}}{m_{b}}+\frac{m_{h} R^{2}}{I_{b}}+1\right)}\right] \\
\omega_{b}=\frac{m_{h} u_{h} P}{I_{b}}\left[1-\frac{m_{h}\left(\frac{1}{m_{b}}+\frac{R^{2}}{I_{b}}\right)-1}{\left(\frac{m_{h}}{m_{b}}+\frac{m_{h} R^{2}}{I_{b}}+1\right)}\right]
\end{gathered}
$$

As noted previously, it was important to design an experiment that reduced losses. However, the experimental data could not match theoretical perfectly due to losses from friction in the firing mechanism hinge, friction between the block and table, and the drag force. A simple analysis of energy loss of the firing mechanism showed how much friction affects the actual velocity of the hammer just before impact compared to the frictionless, theoretical value.

To determine velocity of the hammer experimentally, the high-speed camera was placed perpendicular to the plane in which the hammer traveled. 15 runs were conducted to determine the average velocity of the hammer during impact. For each run, a single velocity was recorded at the point of impact (Figure 5.13). All 15 points are averaged together to determine a velocity of $183.4 \mathrm{~cm} / \mathrm{sec}$. Table 5-3 shows the values recorded for each run as well as percent loss of velocity and energy, where energy was determined by the equation,

$$
E_{h}=\frac{1}{2} m_{h} v_{h}^{2}
$$

From Table 5-3, the nearly 22 percent loss in velocity is relatively high. It is recommended that future experiments use a high quality bearing to reduce friction to lower the percent loss. 


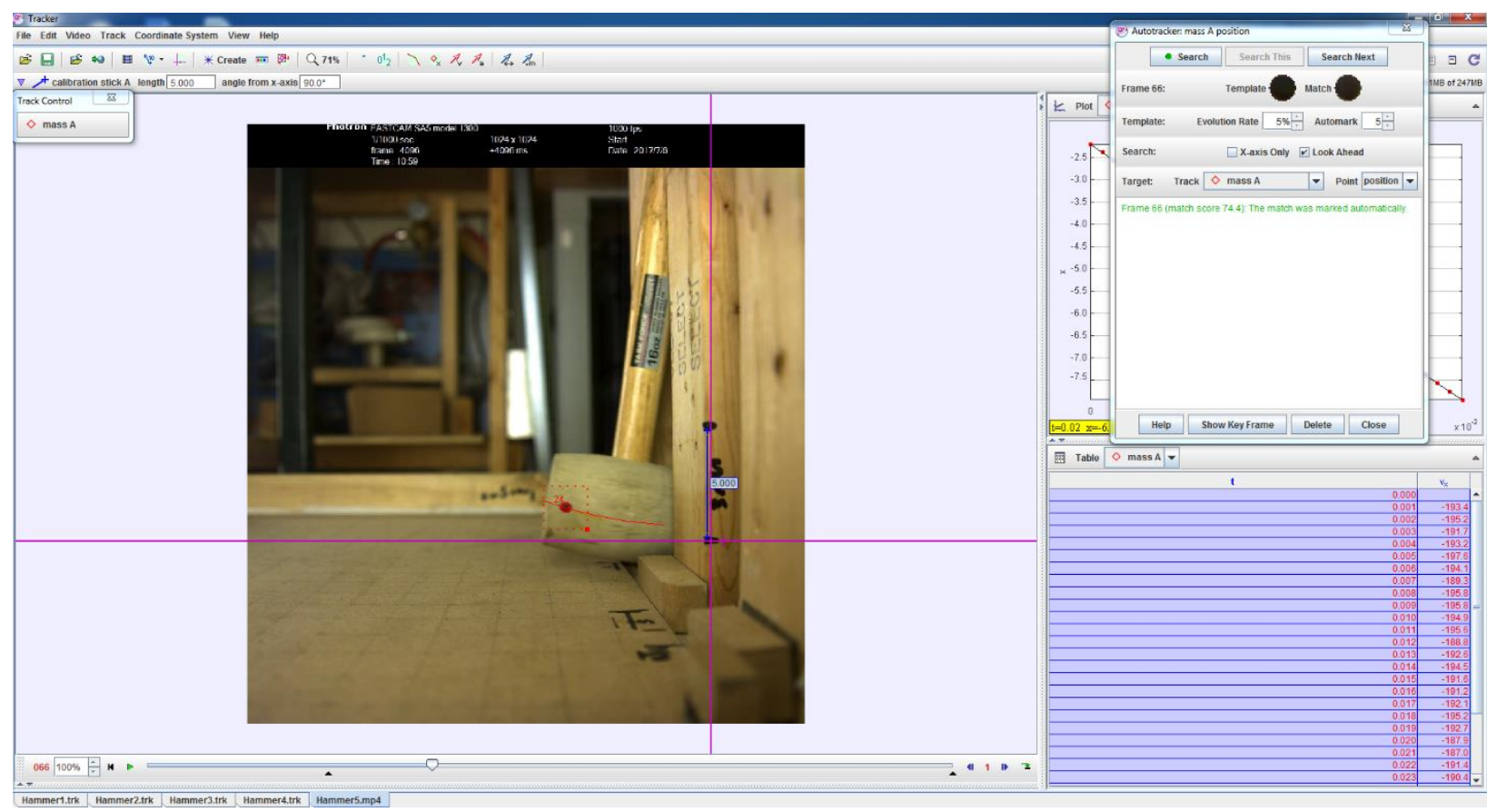

Figure 5.13: Experimental hammer velocity to determine loss due to friction and drag. One data point was taken for 15 runs and averaged together to determine hammer velocity.

Table 5-3: Hammer energy loss due to friction and drag.

\begin{tabular}{|c|c|c|c|c|c|c|c|}
\hline \multicolumn{8}{|c|}{ Hammer Velocity Measurement $\left(u_{h 1}\right)$} \\
\hline $\begin{array}{c}\text { Test } 1 \\
{[\mathrm{~cm} / \mathrm{sec}]}\end{array}$ & $\begin{array}{c}\text { Test } 2 \\
{[\mathrm{~cm} / \mathrm{sec}]}\end{array}$ & $\begin{array}{c}\text { Test } 3 \\
{[\mathrm{~cm} / \mathrm{sec}]}\end{array}$ & $\begin{array}{c}\text { Test } 4 \\
{[\mathrm{~cm} / \mathrm{sec}]}\end{array}$ & $\begin{array}{c}\text { Test } 5 \\
{[\mathrm{~cm} / \mathrm{sec}]}\end{array}$ & $\begin{array}{c}\text { Test } 6 \\
{[\mathrm{~cm} / \mathrm{sec}]}\end{array}$ & $\begin{array}{c}\text { Test } 7 \\
{[\mathrm{~cm} / \mathrm{sec}]}\end{array}$ & $\begin{array}{c}\text { Test } 8 \\
{[\mathrm{~cm} / \mathrm{sec}]}\end{array}$ \\
\hline 180.7 & 185.4 & 179.3 & 186.8 & 184.8 & 183.3 & 182.2 & 189.1 \\
\hline $\begin{array}{c}\text { Test } 9 \\
{[\mathrm{~cm} / \mathrm{sec}]}\end{array}$ & $\begin{array}{c}\text { Test } 10 \\
{[\mathrm{~cm} / \mathrm{sec}]}\end{array}$ & $\begin{array}{c}\text { Test } 11 \\
{[\mathrm{~cm} / \mathrm{sec}]}\end{array}$ & $\begin{array}{c}\text { Test } 12 \\
{[\mathrm{~cm} / \mathrm{sec}]}\end{array}$ & $\begin{array}{c}\text { Test } 13 \\
{[\mathrm{~cm} / \mathrm{sec}]}\end{array}$ & $\begin{array}{c}\text { Test } 14 \\
{[\mathrm{~cm} / \mathrm{sec}]}\end{array}$ & $\begin{array}{c}\text { Test } 15 \\
{[\mathrm{~cm} / \mathrm{sec}]}\end{array}$ & \\
\hline 183.8 & 184.4 & 181.8 & 181.8 & 182.5 & 179.6 & 186.0 & \\
\hline \multicolumn{3}{|c|}{$\begin{array}{c}\text { Average Measured Hammer } \\
\text { Velocity }[\mathrm{cm} / \mathrm{sec}]\end{array}$} & \multicolumn{3}{|c|}{$\begin{array}{c}\text { Theoretical Hammer } \\
\text { Velocity }[\mathrm{cm} / \mathrm{sec}]\end{array}$} & \multicolumn{2}{|c|}{$\begin{array}{l}\text { Percent Velocity } \\
\text { Loss }\end{array}$} \\
\hline \multicolumn{3}{|c|}{184.0} & \multicolumn{3}{|c|}{234} & \multicolumn{2}{|c|}{$21.39 \%$} \\
\hline \multicolumn{3}{|c|}{$\begin{array}{c}\text { Average Final Hammer } \\
\text { Energy Measured } \\
{[\mathrm{J}]} \\
\end{array}$} & \multicolumn{3}{|c|}{$\begin{array}{c}\text { Theoretical Final Hammer } \\
\text { Energy } \\
{[\mathbf{J}]}\end{array}$} & \multicolumn{2}{|c|}{$\begin{array}{c}\text { Percent Energy } \\
\text { Loss }\end{array}$} \\
\hline \multicolumn{3}{|c|}{1.1} & \multicolumn{3}{|c|}{1.7} & \multicolumn{2}{|c|}{$38.20 \%$} \\
\hline
\end{tabular}


After the friction loss in the hammer was analyzed, the blocks were tested to evaluate their velocities. Using the average translational and rotational velocities from Table 5-4 and Table 5-5, the average values over the three runs were calculated and are shown in Table 5-6.

Table 5-4: Horizontal test block translational velocities collected from Tracker software and averaged in Excel.

\begin{tabular}{|c|c|c|c|c|c|}
\hline & \multicolumn{5}{|c|}{$\mathrm{U}_{\mathrm{x} \_ \text {average }}[\mathrm{cm} / \mathrm{sec}]$} \\
\hline & \multirow{2}{*}{ Run } & \multicolumn{4}{|c|}{ Size } \\
\hline & & Block 1 & Block 2 & Block 3 & Block 4 \\
\hline \multirow{3}{*}{ MMOI 1} & 1 & 133 & 141 & 135 & 138 \\
\hline & 2 & 148 & 116 & 137 & 141 \\
\hline & 3 & 147 & 145 & 119 & 154 \\
\hline \multirow{3}{*}{ MMOI 2} & 1 & 155 & 139 & 130 & 141 \\
\hline & 2 & 147 & 142 & 129 & 149 \\
\hline & 3 & 158 & 147 & 147 & 137 \\
\hline \multirow{3}{*}{ MMOI 3} & 1 & 152 & 131 & 132 & 167 \\
\hline & 2 & 149 & 151 & 134 & 188 \\
\hline & 3 & 173 & 137 & 130 & 187 \\
\hline
\end{tabular}

Table 5-5: Horizontal test block rotational velocities collected from Tracker software and averaged in Excel.

\begin{tabular}{|l|c|c|c|c|c|}
\hline \multirow{2}{*}{} & \multicolumn{5}{|c|}{ Waverage [deg/sec] } \\
\cline { 2 - 6 } & \multirow{2}{*}{ Run } & \multicolumn{5}{c|}{ Size } \\
\cline { 2 - 6 } & & Block 1 & Block 2 & Block 3 & Block 4 \\
\hline \multirow{3}{*}{ MMOI 1 } & 1 & 469 & 316 & 176 & 625 \\
\cline { 2 - 6 } & 2 & 538 & 246 & 177 & 658 \\
\cline { 2 - 6 } & 3 & 536 & 309 & 156 & 700 \\
\hline \multirow{3}{*}{ MMOI 2 } & 1 & 686 & 242 & 138 & 541 \\
\cline { 2 - 6 } & 2 & 619 & 251 & 131 & 558 \\
\hline \multirow{3}{*}{ MMOI 3 } & 3 & 682 & 253 & 157 & 528 \\
\cline { 2 - 6 } & 2 & 734 & 168 & 103 & 490 \\
\cline { 2 - 6 } & 3 & 821 & 183 & 106 & 523 \\
\hline
\end{tabular}


Table 5-6: Translational and rotational velocities used for dimensionless term calculations. Average of values over three runs.

\begin{tabular}{|c|c|c|c|}
\hline \multirow{4}{*}{ Block 1 } & & $\begin{array}{c}\mathbf{U}_{\mathbf{x}_{\text {_average }}} \\
{[\mathrm{cm} / \mathrm{sec}]}\end{array}$ & $\begin{array}{c}\boldsymbol{\omega}_{\text {average }} \\
{[\mathrm{rad} / \mathrm{sec}]}\end{array}$ \\
\cline { 3 - 4 } & MMOI 1 & 138.6 & 8.5 \\
\cline { 2 - 4 } & MMOI 2 & 152.0 & 11.3 \\
\hline \multirow{3}{*}{ Block 2 } & MMOI 3 & 154.3 & 12.8 \\
\cline { 2 - 4 } & MMOI 1 & 145.3 & 5.5 \\
\cline { 2 - 4 } & MMOI 3 & 141.5 & 4.3 \\
\hline \multirow{3}{*}{ Block 3 } & MMOI 1 & 135.9 & 3.1 \\
\cline { 2 - 4 } & MMOI 2 & 133.3 & 3.1 \\
\cline { 2 - 4 } & MMOI 3 & 130.3 & 2.4 \\
\hline \multirow{3}{*}{ Block 4 } & MMOI 1 & 144.1 & 11.8 \\
\cline { 2 - 4 } & MMOI 2 & 142.2 & 9.5 \\
\cline { 2 - 4 } & MMOI 3 & 180.2 & 8.9 \\
\hline
\end{tabular}

The data calculated in Table 5-6 was used to calculate dimensionless angular velocity for different length scales. Each length scale used was plotted to determine if a scaling relationship was present. Some length scales proved to be more useful than others. Based on data from Figure 5.14 through Figure 5.19, the length scale of surface area divided by the dimension $L$ appears to be the most appropriate selection. This length scale, shows signs of following a power or logarithmic function. 


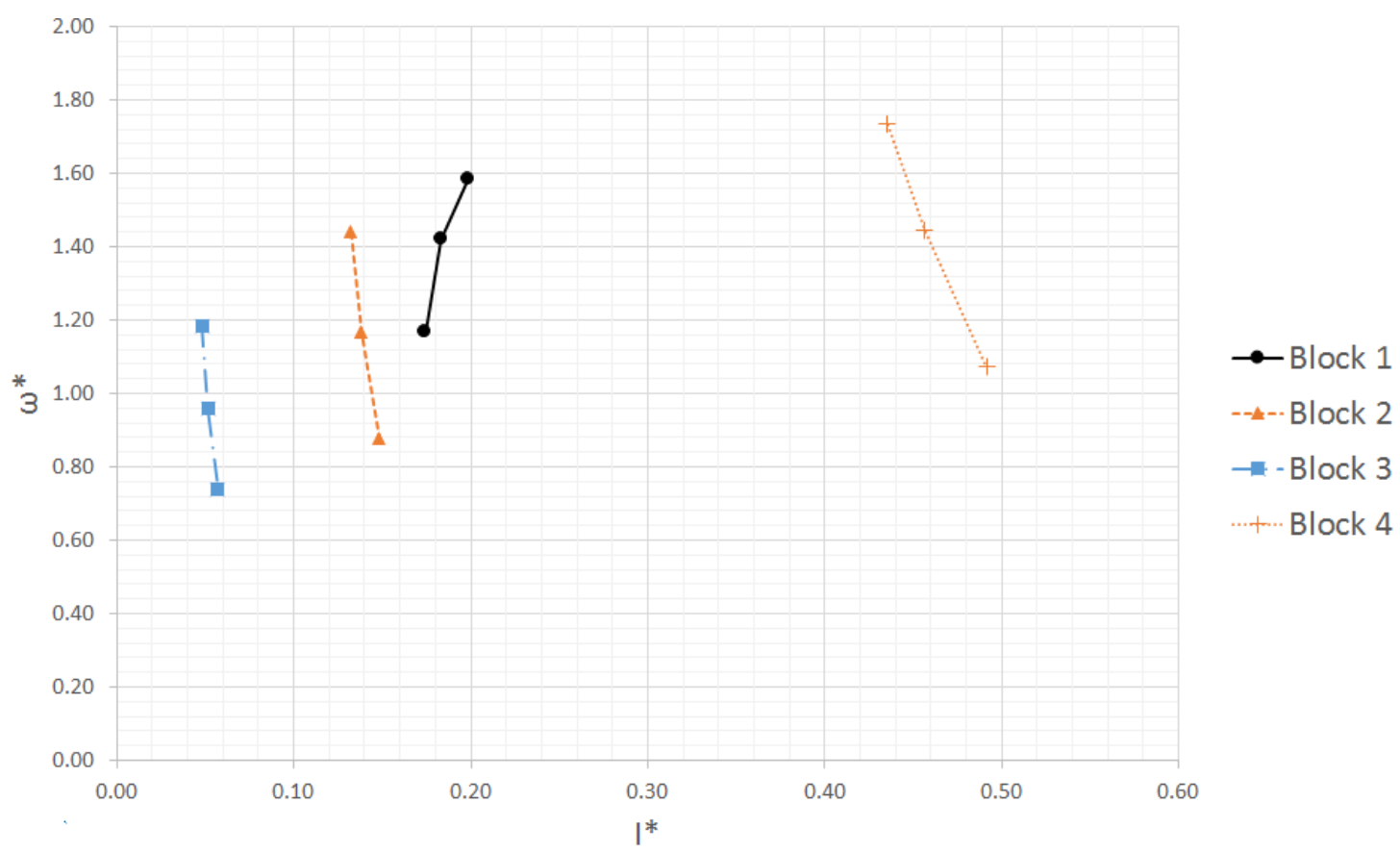

Figure 5.14: Length scale of hydraulic diameter

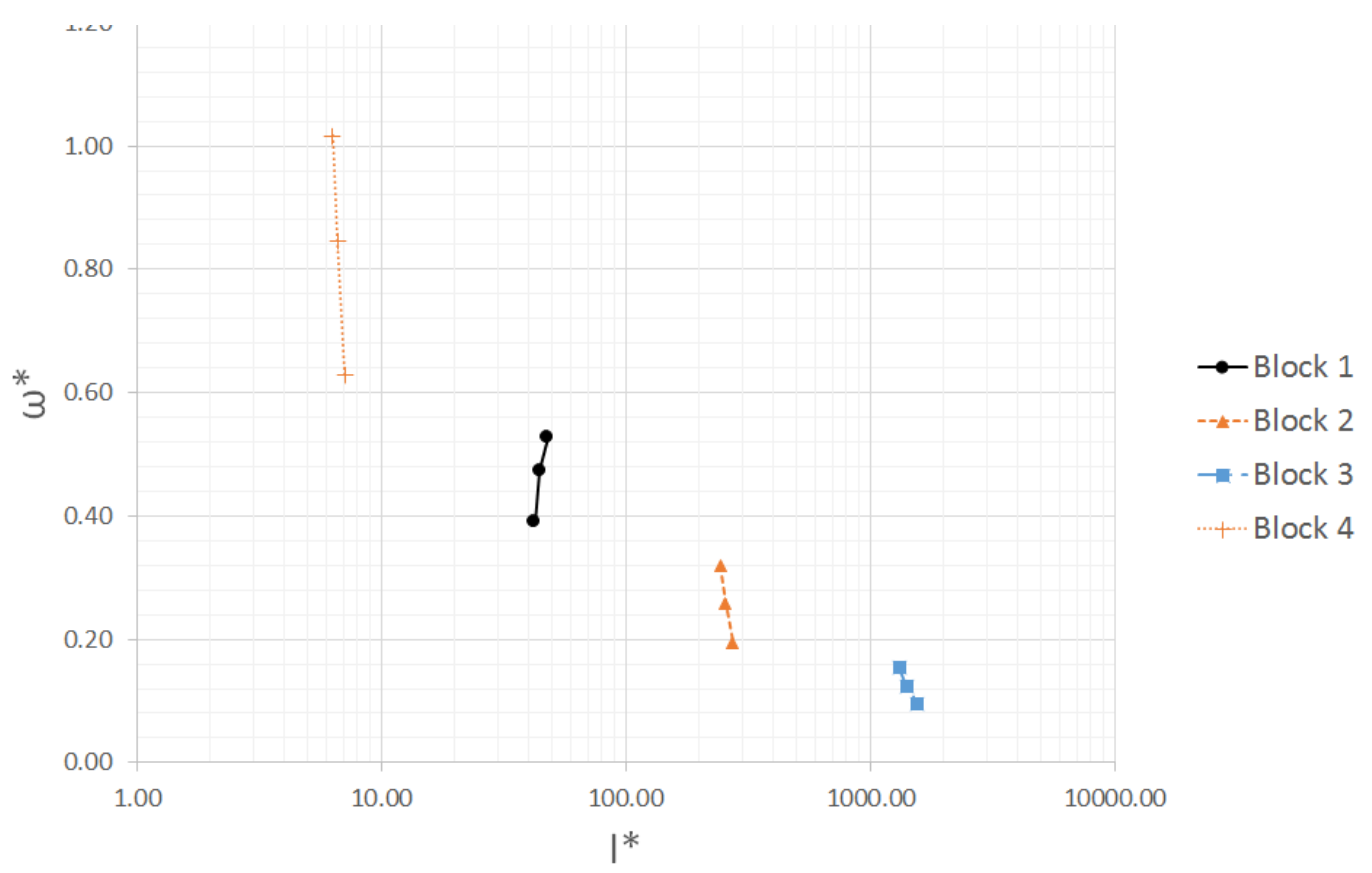

Figure 5.15: Length scale of surface area divided by $L$. 


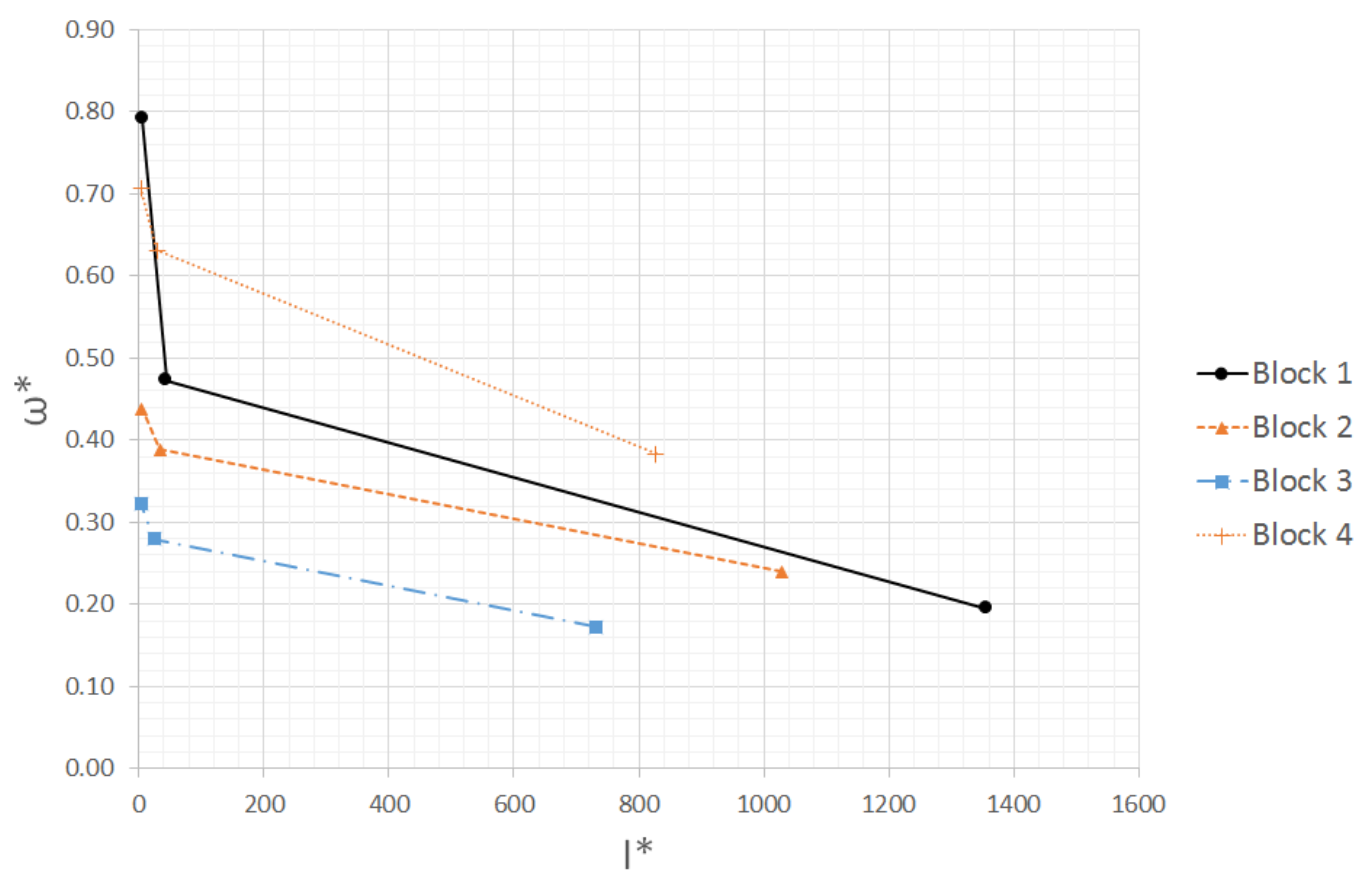

Figure 5.16: Length scale of length to bolt.

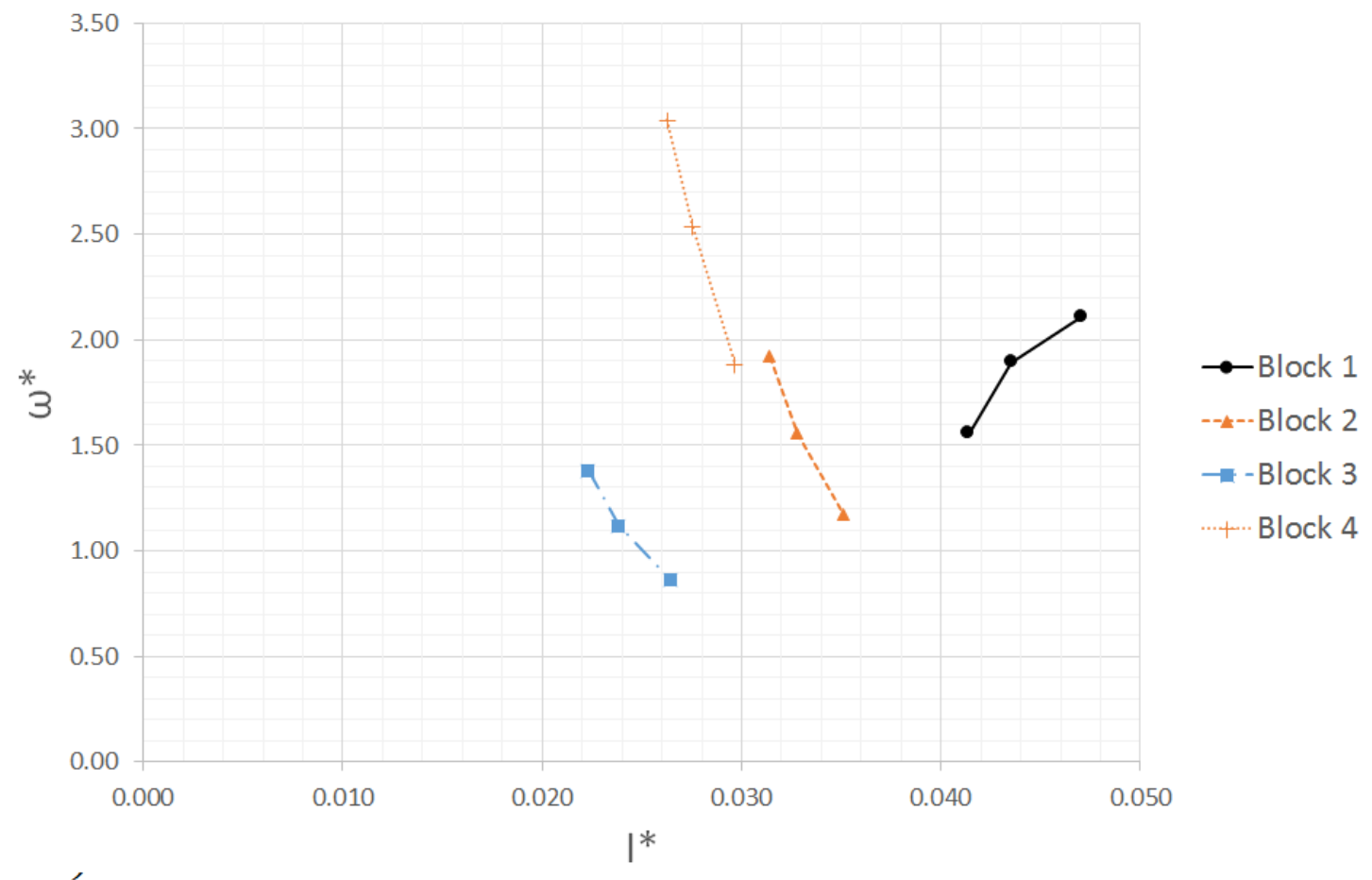

Figure 5.17: Length scale of dimension W 


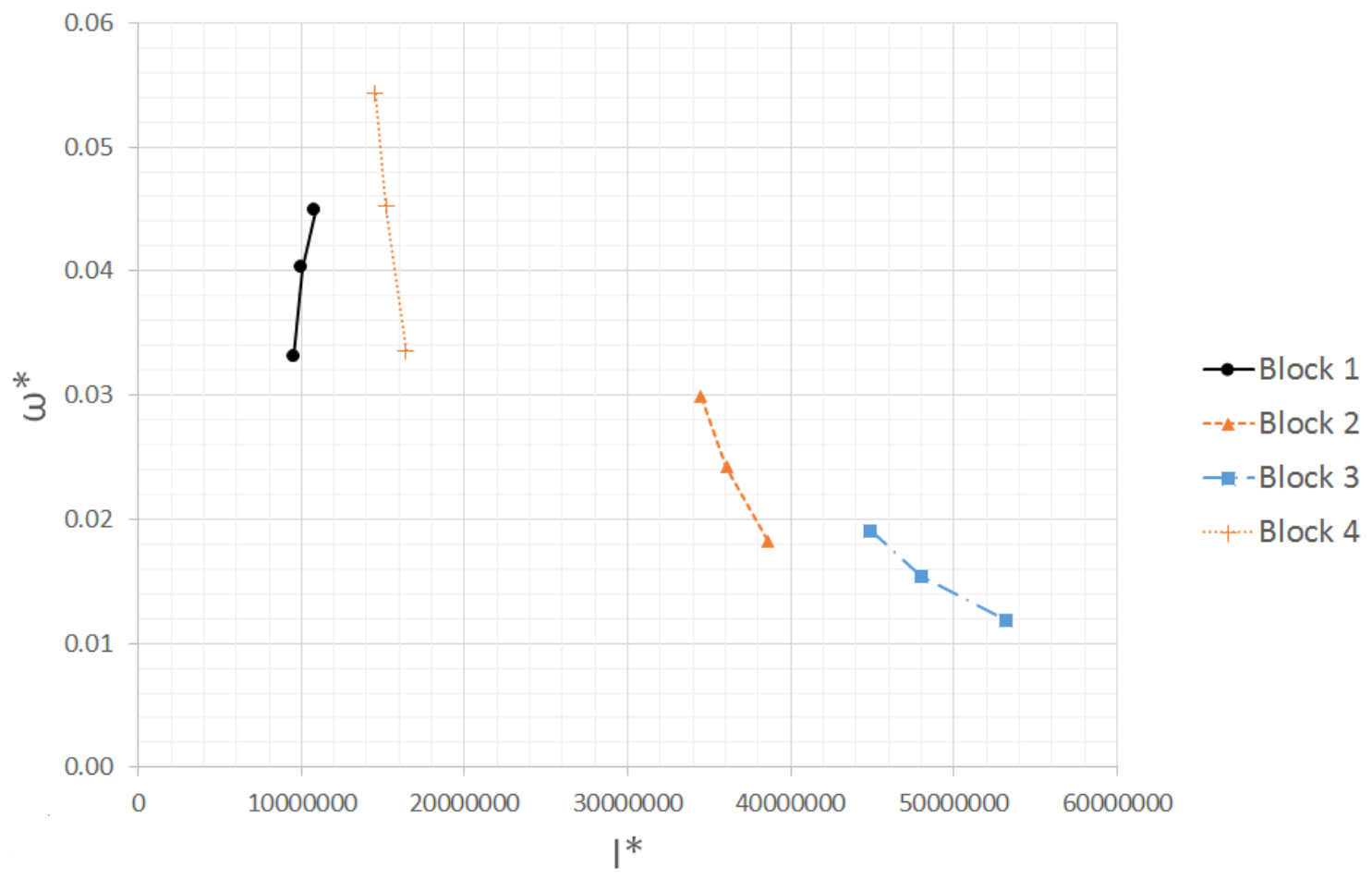

Figure 5.18: Length scale of volume divided by total surface area.

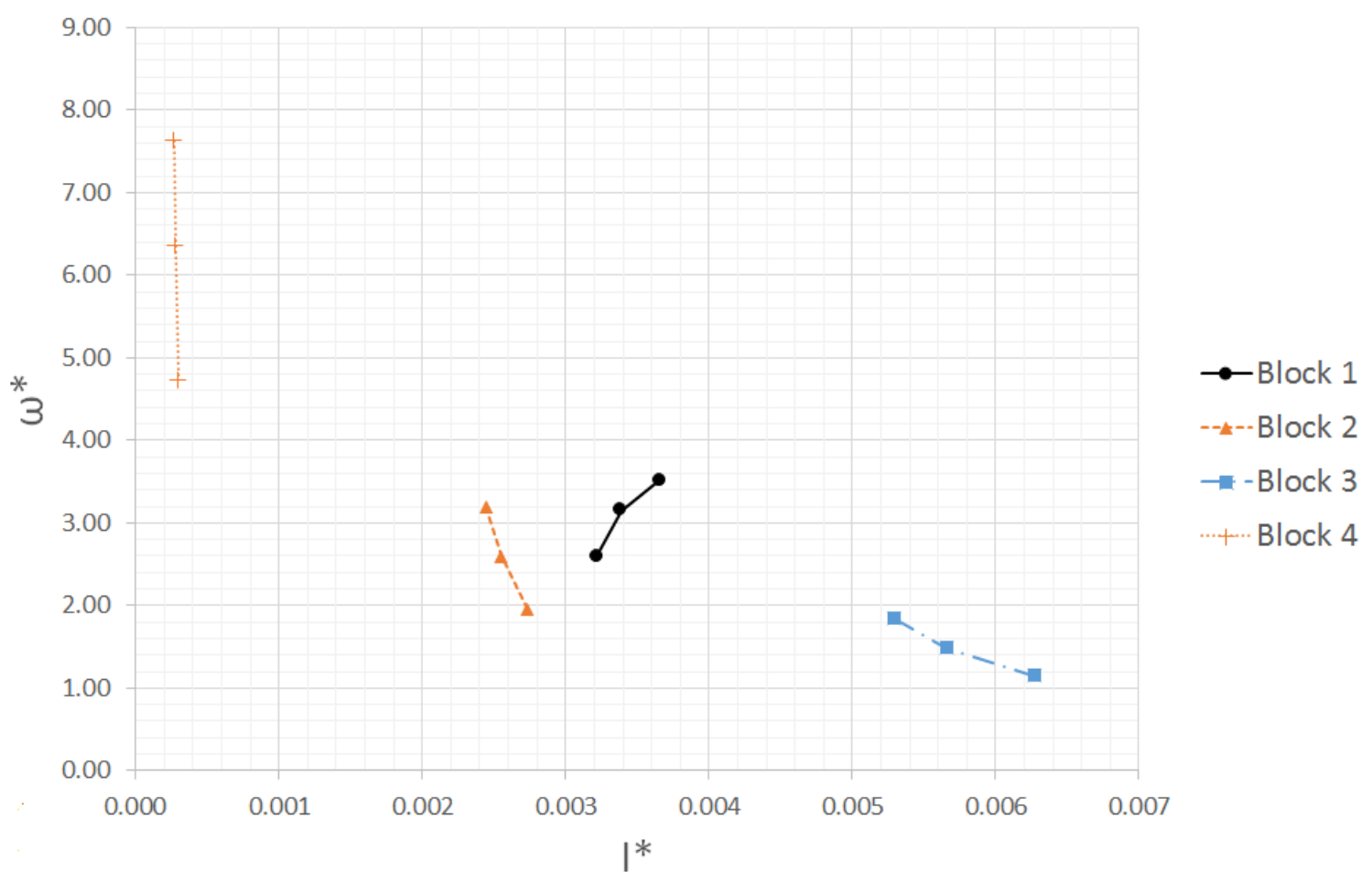

Figure 5.19: Length scale of dimension W multiplied by aspect ratio. 
It was desirable to compare experimental data to theoretical calculations to determine the general trend followed by the data. Using Equation 5-14, Equation 5-15, and Equation 5-16 derived previously, the experimental data was compared to theoretical. First, more data points were created by applying a line of best fit between the current three MMOI data points. A plot of bolt location in centimeters versus MMOI for each block size was created to curve fit the line (Figure 5.20). Equations are provided in Table 5-7.

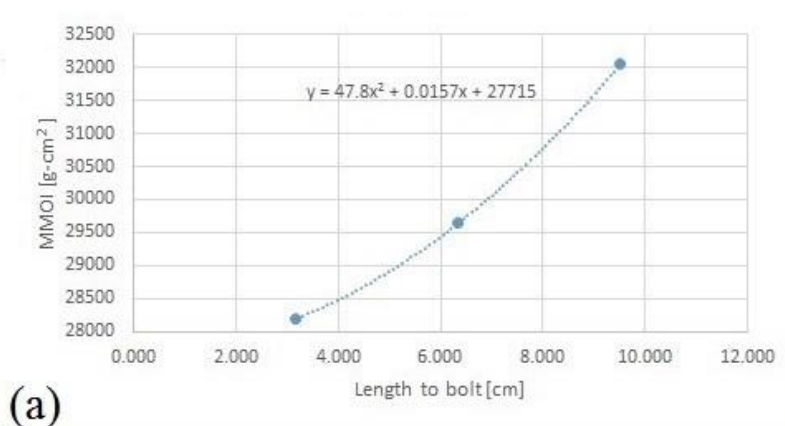

(a)

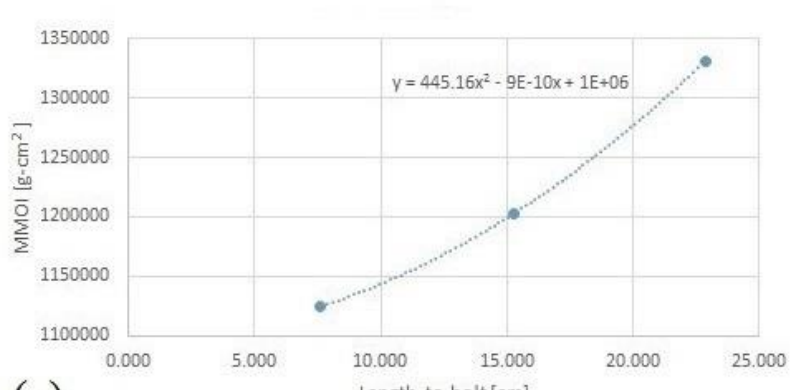

(c)

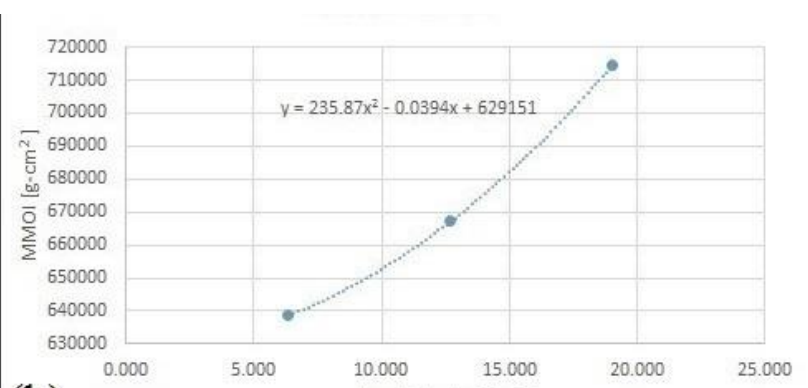

(b)

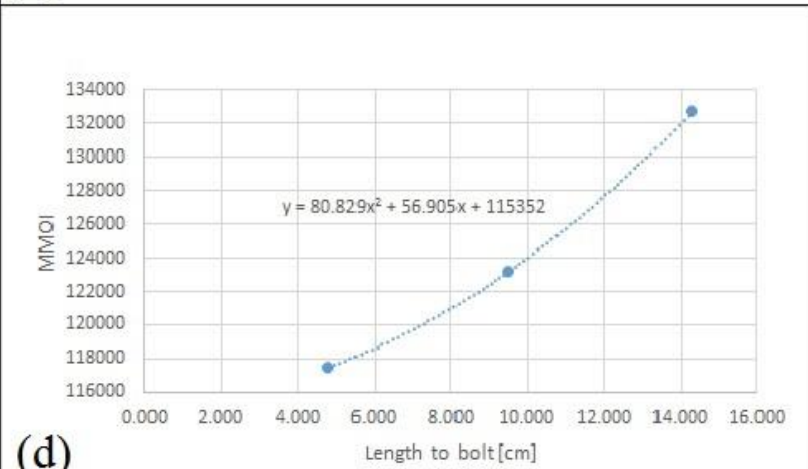

Figure 5.20: Curve fit equation to interpolate more MMOI data points. Data is used to compare experiential data to theoretical. (a) Block 1. (b) Block 2. (c) Block 3. (d) Block 4.

Table 5-7: Trendline equations from Figure 5.20. Equations used to interpolate more MMOI values to compare to

\begin{tabular}{|c|c|}
\hline \multicolumn{2}{|c|}{ experimental data. } \\
\hline Block Size & Trendline Equation \\
\hline Block 1 & $47.8 x^{2}+0.0157 x+27715$ \\
\hline Block 2 & $235.87 x^{2}-0.0394 x+628151$ \\
\hline Block 3 & $445.16 x^{2}-\left(9 \times 10^{-10}\right) x+\left(1 \times 10^{6}\right)$ \\
\hline Block 4 & $80.829 x^{2}+56.905 x+115352$ \\
\hline
\end{tabular}

Using the curved fit equations in Table 5-7, a series of MMOI values were interpolated to better portray the theoretical data. Table 5-8 shows an example of data used to plot theoretical data; where MMOI 1 corresponds to a bolt location $1 \mathrm{~cm}$ from the CG, MMOI 2 corresponds to a bolt location $2 \mathrm{~cm}$ from the $\mathrm{CG}$, etc. Dimensionless MMOI and dimensionless angular velocity 
were calculated using Equation 5-1 and Equation 5-2, respectively, while $u_{b}$ and $\omega_{b}$ were calculated using Equation 5-15 and Equation 5-16, respectively. The data in Table 5-8 is based on maintaining the same torque on the block as the MMOI is increased. That is, as the bolts theoretically move away from the CG, the geometry of the block, and the impact location from the hammer, remain constant. In real application, this is not feasible due to the theoretical bolt placement being outside the geometry of the block.

Table 5-8: Interpolated values for comparison of theoretical and experimental data using Block 1 size as an

\begin{tabular}{|c|c|c|c|c|c|}
\hline Block 1 & $\mathrm{I}\left(\mathrm{g}-\mathrm{cm}^{2}\right)$ & $\mathrm{I}^{*}$ & $\begin{array}{c}\text { example. } \\
(\mathrm{cm} / \mathrm{sec})\end{array}$ & $\begin{array}{c}\omega \_ \text {avg } \\
(\mathrm{rad} / \mathrm{sec})\end{array}$ & $\begin{array}{c}\text { Reduced Angular } \\
\text { Velocity }\end{array}$ \\
\hline MMOI 1 & 27763 & 41.7 & 312 & 11 & 0.22 \\
\hline MMOI 2 & 27906 & 42.0 & 312 & 11 & 0.22 \\
\hline MMOI 3 & 28145 & 42.3 & 313 & 11 & 0.22 \\
\hline MMOI 4 & 28480 & 42.8 & 314 & 11 & 0.21 \\
\hline MMOI 5 & 28910 & 43.5 & 315 & 10 & 0.21 \\
\hline MMOI 6 & 29436 & 44.3 & 316 & 10 & 0.21 \\
\hline MMOI 7 & 30057 & 45.2 & 318 & 10 & 0.20 \\
\hline MMOI 8 & 30774 & 46.3 & 319 & 10 & 0.20 \\
\hline MMOI 9 & 31587 & 47.5 & 321 & 10 & 0.19 \\
\hline MMOI 10 & 32495 & 48.9 & 323 & 9 & 0.19 \\
\hline MMOI 11 & 33499 & 50.4 & 325 & 9 & 0.18 \\
\hline MMOI 12 & 34598 & 52.0 & 327 & 9 & 0.18 \\
\hline MMOI 13 & 35793 & 53.8 & 329 & 9 & 0.17 \\
\hline MMOI 14 & 37084 & 55.8 & 331 & 9 & 0.16 \\
\hline MMOI 15 & 38470 & 57.8 & 334 & 8 & 0.16 \\
\hline MMOI 16 & 39952 & 60.1 & 336 & 8 & 0.15 \\
\hline MMOI 17 & 41529 & 62.4 & 338 & 8 & 0.15 \\
\hline MMOI 18 & 43202 & 65.0 & 340 & 8 & 0.14 \\
\hline MMOI 19 & 44971 & 67.6 & 343 & 7 & 0.13 \\
\hline MMOI 20 & 46835 & 70.4 & 345 & 7 & 0.13 \\
\hline MMOI 21 & 48795 & 73.4 & 347 & 7 & 0.12 \\
\hline MMOI 22 & 50851 & 76.5 & 349 & 7 & 0.12 \\
\hline MMOI 23 & 53002 & 79.7 & 351 & 6 & 0.11 \\
\hline MMOI 24 & 55248 & 83.1 & 353 & 6 & 0.11 \\
\hline MMOI 25 & 57590 & 86.6 & 355 & 6 & 0.11 \\
\hline
\end{tabular}

From the data in Table 5-8, a plot was created to compare the experimental values for dimensionless terms to theoretical values (Figure 5.21). The theoretical data does not match 
experimental data due to factors such as drag, friction, impulse of the impact, and other losses within the system. The theoretical data was used to represent ideal conditions for the experiment so a comparison could be made directly. If the experimental data did not follow a similar pattern as the theoretical, further analysis would be conducted to determine what factors were disrupting the data.

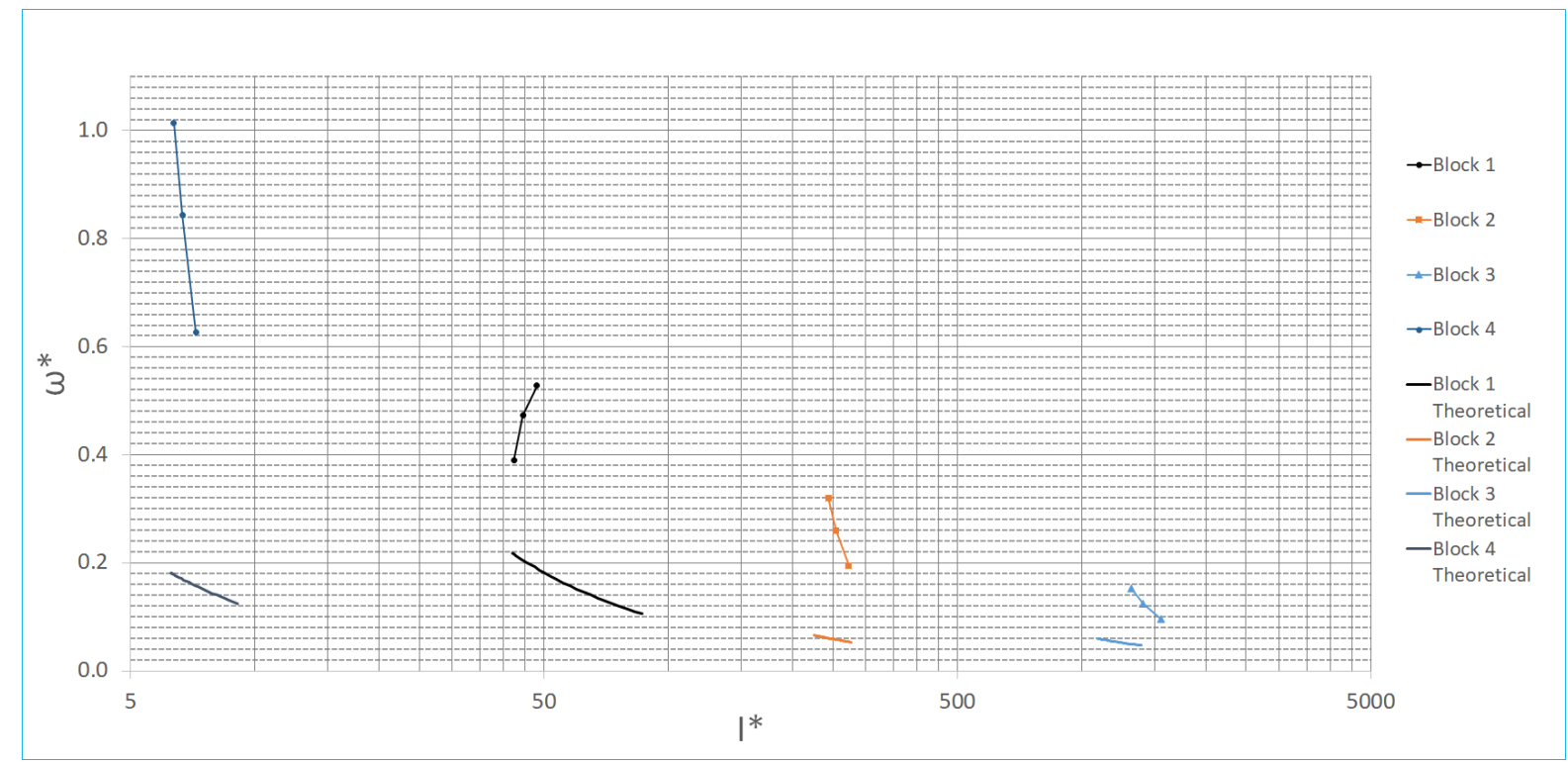

Figure 5.21: Semi-log plot comparing experimental and theoretical data for dimensionless MMOI and dimensionless angular velocity of block.

It is difficult to determine if a scaling relationship exists from only data in Figure 5.21. To better understand the length scale, it was applied to different testing methods as well. An observation that can be made from Figure 5.21 is that a higher aspect ratio seems to increase the range over which dimensionless angular velocity can occur. Additionally, the slope of the experimental data is significantly higher than that of the theoretical data. This can only be due to the difference in the velocity ratios, since the length scale does not change. For the experimental data, the angular velocity must increase more relative to the translational velocity or the translational velocity must decrease. It is a better assumption that drag on the block causes a lower translational velocity than the theoretical calculation. This also explains why the slop of the experimental data increases as the size increases.

To better understand which factors influence translational and rotational velocity of the block, an ANOVA analysis was performed on the data. A two-factor analysis with replication and an alpha value of 0.05 , was used due to the two factors analyzed in the test, MMOI and size. Using 
an ANOVA analysis with replication also accounts for interaction between the two factors. The alpha value represents the sensitivity for the null hypothesis (i.e. no interaction between variables) to be rejected. Table 5-4 and Table 5-5 show the collected data for each run for translational velocity and angular velocity, respectively.

The two-way ANOVA analysis gave insight on how factors such as block size, MMOI, and interaction between the factors affects the outcome. For example, by looking at the tables, it can be assumed that block size greatly affects the angular velocity, but it is difficult to determine the effects on translational velocity by inspection alone. In the ANOVA tables, the variation statistic, $F$, is a ratio of values within the table, while critical variation statistic, $F$ crit, is the maximum ratio before the null hypothesis is rejected and a significant effect on data is noted. Each of these variables were used to determine influence of each variable. The three main sources of influence within the data are Sample, representing interaction between rows (i.e. MMOI 1, MMOI 2, and MMOI 3); Columns representing interaction between block sizes in the columns; and Interaction, combining values between the rows and columns.

Table 5-9: ANOVA analysis of translational velocity data from Table 5-4.

\begin{tabular}{|ccccccc|}
\hline Source of Variation & $S S$ & $d f$ & $M S$ & $F$ & P-value & $F$ crit \\
\hline Sample (MMOI) & 1309.1 & 2 & 654.6 & 7.4 & $3.2 \mathrm{E}-03$ & 3.4 \\
Column (Geometry) & 3169.0 & 3 & 1056.3 & 11.9 & $5.7 \mathrm{E}-05$ & 3.0 \\
Interaction & 2032.0 & 6 & 338.7 & 3.8 & $8.2 \mathrm{E}-03$ & 2.5 \\
Within & 2129.3 & 24 & 88.7 & & & \\
& & & & & & \\
Total & 8639.5 & 35 & & & & \\
\hline
\end{tabular}

To reject the null hypothesis, $F>F$ cri or $P$-value $<$ alpha. Thus, size, MMOI, and interaction all affect the translational velocity of the block, but size has the greatest influence. This is expected due to the increase in mass of the block while the hammer mass remains constant. Additionally, interaction has the least influence on the translational velocity. 
Table 5-10: ANOVA analysis of rotational velocity data from Table 5-5.

\begin{tabular}{|ccccccc|}
\hline Source of Variation & $S S$ & $d f$ & $M S$ & $F$ & P-value & F crit \\
\hline Sample (MMOI) & 2454.3 & 2 & 1227.2 & 1.4 & $2.6 \mathrm{E}-01$ & 3.4 \\
Column (Geometry) & 1641478.7 & 3 & 547159.6 & 637.2 & $5.3 \mathrm{E}-23$ & 3.0 \\
Interaction & 148100.8 & 6 & 24683.5 & 28.7 & $7.9 \mathrm{E}-10$ & 2.5 \\
Within & 20607.0 & 24 & 858.6 & & & \\
& & & & & & \\
Total & 1812640.8 & 35 & & & & \\
\hline
\end{tabular}

For the angular velocity, MMOI alone can be rejected as a significant variable. Additional points or heavier weights could possible affect the angular velocity. Relatively small increases in the MMOI were shown to have no significant effect on the data. However, the larger increase in MMOI caused by changing the size of the block greatly affected the result. The relatively high $F$ value compared to $F$ crit indicates that size is the most important factor to change angular velocity. Again, this was expected due to the increasing mass of the block between sizes, but as a quantity, it is easier to see how much more importance it carries.

\subsection{D Vertical Plane}

Testing in the vertical plane consisted of four foam blocks, each with three options for MMOI, being dropped from a height of $243 \mathrm{~cm}$. The blocks were recorded at $500 \mathrm{fps}$ with a shutter speed of $1 / 1000$. The purpose of this test was to further examine the relationship between dimensionless MMOI and dimensionless angular velocity, when the block is only acted upon by gravity. The block tended to start a tumbling motion after falling approximately $100 \mathrm{~cm}$. To help quantify 3D motion, a grid of 4"x4" squares was used to determine how different block MMOIs and sizes affected the tendency to induce tumble. A GoPro Hero 3 was used to help determine impact location in a plane perpendicular to the initial position of the block. Using Tracker, values for linear and angular velocity were calculated numerically, based on user defined locations and camera parameters.

Since some blocks tended to tumble before reaching the floor, the full trajectory of the blocks was not successfully tracked. For some runs, less than half of the total trajectory was recorded before the tracking makers rotated out of view. Therefore, average translational velocity for each configuration of size and MMOI was calculated using three methods and comparing 
results. Average velocity calculations for each method can be seen in Table 5-11. First, the average velocity was calculated by comparing the total drop distance traveled by each block size.

$$
u_{\text {avg }}=\frac{x_{\text {total }}}{t_{\text {total }}}
$$

Where $x$ is the total distance traveled, $243 \mathrm{~cm}$, and $t$ is the time from when the block was released to when it contacted the ground. Time, $t$, was determined by visually inspecting the high-speed video, and noting when the block made first contact with the floor. The initial and final frames were used, along with the camera frame rate, to determine the total time of free fall. This method had a high probability to be influenced by drag. Moreover, as the blocks entered a tumbling state, drag was significantly increased, causing the average velocity equation to be more inaccurate for higher drop positions.

To better represent the average velocity of each block, another method was implemented. The second method used a variation of Equation 5-17. However, the time, $t$, used was the six time steps from 0.216 to 0.226 seconds. This time was used because data at this time was available for each run. That is, some runs were evaluated for a longer period of time, but the shortest run tracked was slightly longer than 0.226 seconds; each run was tracked for at least 0.226 seconds. In Tracker, the tape measure tool was used to determine the distance traveled by the $\mathrm{CG}$ of the block from $t=$ 0 to $t=0.226$ seconds (Figure 5.22). The tape measure tool used the scaled distance, defined using the calibration stick, to determine a distance. That distance was recorded at $t=0.226$ for each run and used in Equation 5-17 to calculate average velocity. The measured distance each block traveled accounted for any effects from drag, giving a more accurate calculation of average velocity. The limitation of this method was the relatively short duration of the trajectory use. Additionally, the blocks were not able to reach terminal velocity, and, therefore, continued to accelerate throughout their trajectories.

$$
u_{x}=\frac{x_{\text {measured }}}{t_{\text {tracked }}}
$$




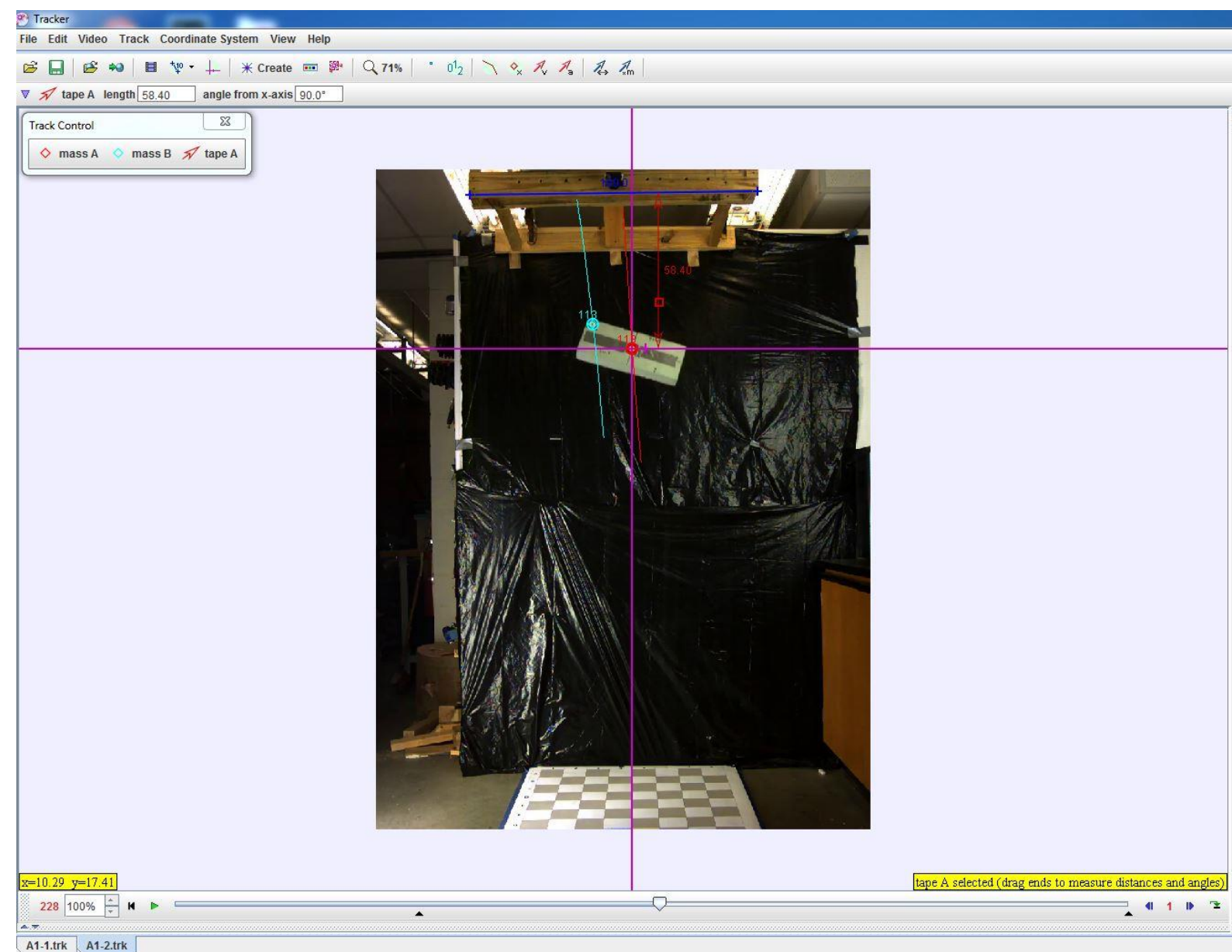

Figure 5.22: Demonstration of Tracker's tape measure tool (indicated by the red arrow) to determine a distance, $x$, for calculating average velocity.

Method three for calculating average velocity followed the findings of Andersen [7]. In which, the average velocity was calculated using the relationship between gravity and quadratic drag (Equation 5-19). Where $\rho_{s}$ is the density of the solid block, $\rho_{f}$ is the density of the surrounding fluid, in this case $0.001204 \mathrm{~g} / \mathrm{cm}^{3}$ for air at $20^{\circ} \mathrm{C}, g$ is the acceleration due to gravity, and $H$ is the thickness dimension of the block.

$$
u_{x}=\sqrt{\left(\frac{\rho_{s}}{\rho_{f}}-1\right) g H}
$$

Equation 5-19

A compilation of the three methods used to analyze the translational velocity is shown in Table 5-11 and their values are shown in Table 5-12. 
Table 5-11: Methods tested to determine an accurate representation of Ux_avearage for drop tests.

\begin{tabular}{|c|c|}
\hline Method & Equation \\
\hline Linear Distance Equation & $u_{x}=\frac{x_{\text {total }}}{t_{\text {total }}}$ \\
\hline Tracker Calibrated Measurement & $u_{x}=\frac{x_{\text {measured }}}{t_{\text {tracked }}}$ \\
\hline $\begin{array}{c}\text { Average Terminal Velocity by Balancing } \\
\text { Gravity with a Quadratic Drag [7] }\end{array}$ & $u_{x}=\sqrt{\left(\frac{\rho_{s}}{\rho_{f}}-1\right) g H}$ \\
\hline
\end{tabular}

Each of the three methods used to calculate average velocity, along with the length scales in Table 5-12, were used to evaluate the dimensionless relationships of MMOI and angular velocity.

Table 5-12: Average translational velocities calculated using the three methods shown as Equation 5-17 through Equation 5-19.

\begin{tabular}{|c|c|c|c|c|}
\hline \multirow{2}{*}{} & \multicolumn{3}{|c|}{$\mathbf{u}_{\mathbf{x}}$} \\
\cline { 3 - 5 } \multicolumn{2}{c|}{} & Equation 5-17 & Equation 5-18 & Equation 5-19 \\
\hline \multirow{3}{*}{ Block 1 } & MMOI 1 & 411.9 & 267.7 & 443.0 \\
\cline { 2 - 5 } & MMOI 2 & 411.9 & 277.0 & 443.0 \\
\cline { 2 - 5 } & MMOI 3 & 405.0 & 256.0 & 443.0 \\
\hline \multirow{3}{*}{ Block 2 } & MMOI 1 & 486.0 & 294.1 & 493.9 \\
\cline { 2 - 5 } & MMOI 2 & 441.8 & 271.5 & 493.9 \\
\cline { 2 - 5 } & MMOI 3 & 441.8 & 293.7 & 493.9 \\
\hline \multirow{3}{*}{ Block 3 } & MMOI 1 & 495.9 & 334.7 & 492.9 \\
\cline { 2 - 5 } & MMOI 2 & 486.0 & 326.5 & 492.9 \\
\cline { 2 - 5 } & MMOI 3 & 495.9 & 316.8 & 492.9 \\
\cline { 2 - 5 } & MMOI 1 & 405.0 & 249.0 & 475.0 \\
\cline { 2 - 5 } & MMOI 2 & 398.4 & 249.4 & 475.0 \\
\hline & MMOI 3 & 405.0 & 262.5 & 475.0 \\
\hline
\end{tabular}

Before plots could be made, Tracker was used to determine angular velocity by first tracking the center of the block until the point of interest was undetectable by the software, due to block tumbling. Then, to determine angular velocity, the corner of the block was tracked with respect to the center over the same time as the previous trajectory. Figure 5.23 shows a situation in which the block rotated into a position where the software was unable to identify the point of interest. Therefore, less than half of the trajectory was used for data collection. Such a short 
duration of the fall would produce inaccurate translational velocities due to the acceleration of the block throughout the fall. However, angular velocity can still be determined over the shorter trajectory since only minimal drag was opposing the rotation of the body as it rotated about its $z$ axis.

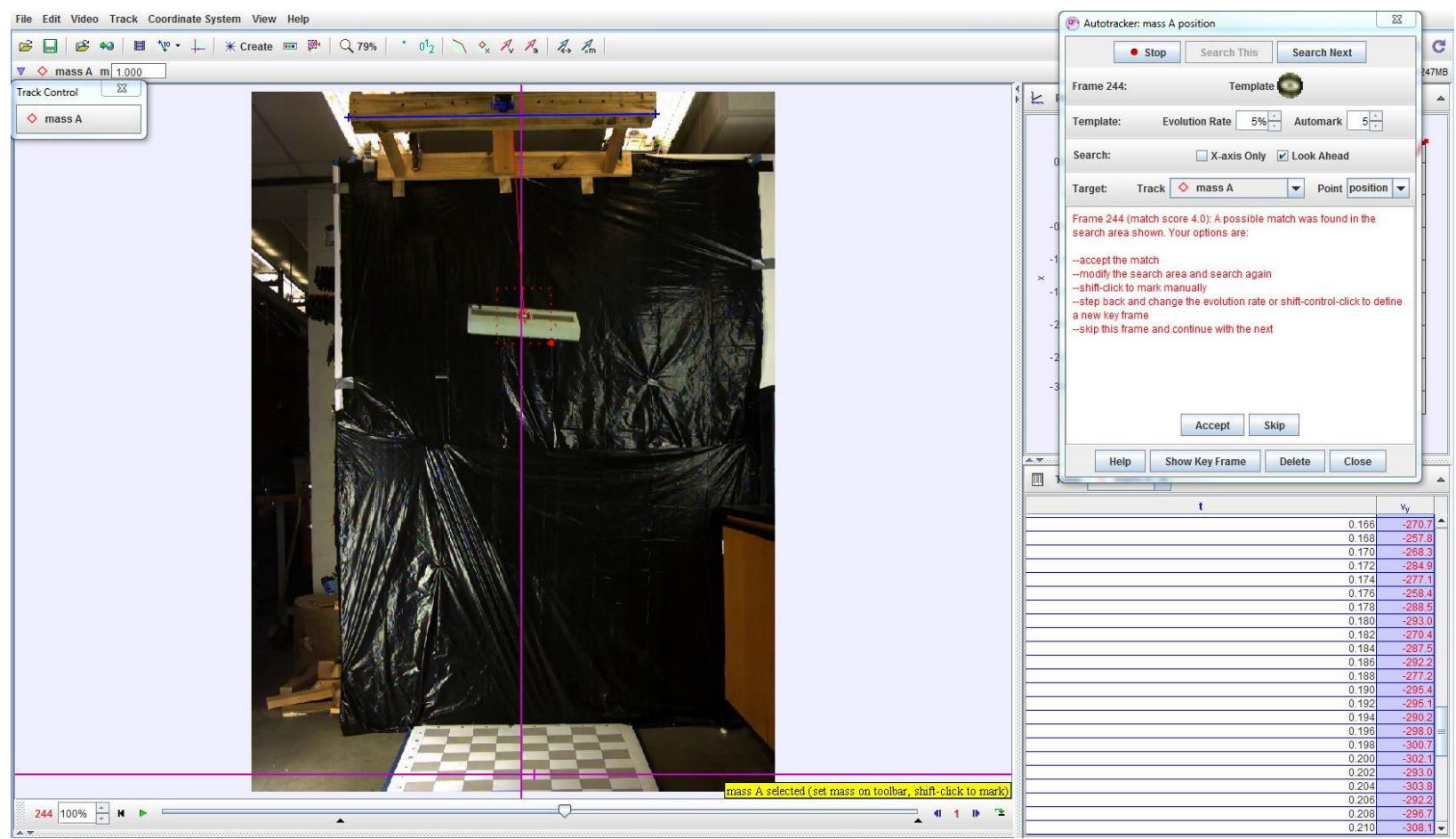

Figure 5.23: Block rotating point of interest out of view. Tracker software unable to determine the point after this frame.

Table 5-13 shows the measured and calculated values used for the analysis. Mass moment of inertia, $I$, comes from the CAD model of the blocks and $\omega \_a v g$ was determined numerically using Tracker data. From these parameters, along with length and mass properties measured previously, dimensionless MMOI and dimensionless angular velocity were calculated and compared. 
Table 5-13: Drop test measured data used for analysis.

\begin{tabular}{|c|c|c|c|c|}
\hline Block 1 & $\mathrm{I}\left(\mathrm{g}-\mathrm{cm}^{2}\right)$ & $\begin{array}{c}\omega \_ \text {avg } \\
(\mathrm{rad} / \mathrm{sec})\end{array}$ & $\mathrm{W}(\mathrm{cm})$ & 25.4 \\
\hline MMOI 1 & 28196.7 & 2.4 & time $(\mathrm{sec})$ & 0.2 \\
\hline MMOI 2 & 29642.3 & 2.2 & $\rho\left(\mathrm{g} / \mathrm{cm}^{3}\right)$ & $6.44 \mathrm{E}-02$ \\
\hline MMOI 3 & 32051.6 & 0.8 & $\mathrm{~g}\left(\mathrm{~cm} / \mathrm{sec}^{2}\right)$ & 981 \\
\hline Block 2 & $\mathrm{I}\left(\mathrm{g}-\mathrm{cm}^{2}\right)$ & $\begin{array}{c}\omega \_ \text {avg } \\
(\mathrm{rad} / \mathrm{sec})\end{array}$ & $\mathrm{W}(\mathrm{cm})$ & 50.8 \\
\hline MMOI 1 & 638661.3 & 1.8 & time (sec) & 0.2 \\
\hline MMOI 2 & 667193.6 & 1.2 & $\rho\left(\mathrm{g} / \mathrm{cm}^{3}\right)$ & $6.01 \mathrm{E}-02$ \\
\hline MMOI 3 & 714747.6 & 1.4 & $\mathrm{~g}\left(\mathrm{~cm} / \mathrm{sec}^{2}\right)$ & 981 \\
\hline Block 3 & $\mathrm{I}\left(\mathrm{g}-\mathrm{cm}^{2}\right)$ & $\begin{array}{c}\omega \_ \text {avg } \\
(\mathrm{rad} / \mathrm{sec})\end{array}$ & $\mathrm{W}(\mathrm{cm})$ & 61.0 \\
\hline MMOI 1 & 1124770.9 & 2.0 & time $(\mathrm{sec})$ & 0.2 \\
\hline MMOI 2 & 1202314 & 1.5 & $\rho\left(\mathrm{g} / \mathrm{cm}^{3}\right)$ & 5.99E-02 \\
\hline MMOI 3 & 1331552.5 & 1.5 & $\mathrm{~g}\left(\mathrm{~cm} / \mathrm{sec}^{2}\right)$ & 981 \\
\hline Block 4 & $\mathrm{I}\left(\mathrm{g}-\mathrm{cm}^{2}\right)$ & $\begin{array}{c}\text { } \begin{array}{c}\text { ang } \\
(\mathrm{rad} / \mathrm{sec})\end{array}\end{array}$ & $\mathrm{W}(\mathrm{cm})$ & 38.1 \\
\hline MMOI 1 & 117487.4 & 0.8 & time $(\mathrm{sec})$ & 0.2 \\
\hline MMOI 2 & 123187.4 & 1.2 & $\rho\left(\mathrm{g} / \mathrm{cm}^{3}\right)$ & 5.57E-02 \\
\hline MMOI 3 & 132694.5 & 0.6 & $\mathrm{~g}\left(\mathrm{~cm} / \mathrm{sec}^{2}\right)$ & 981 \\
\hline
\end{tabular}

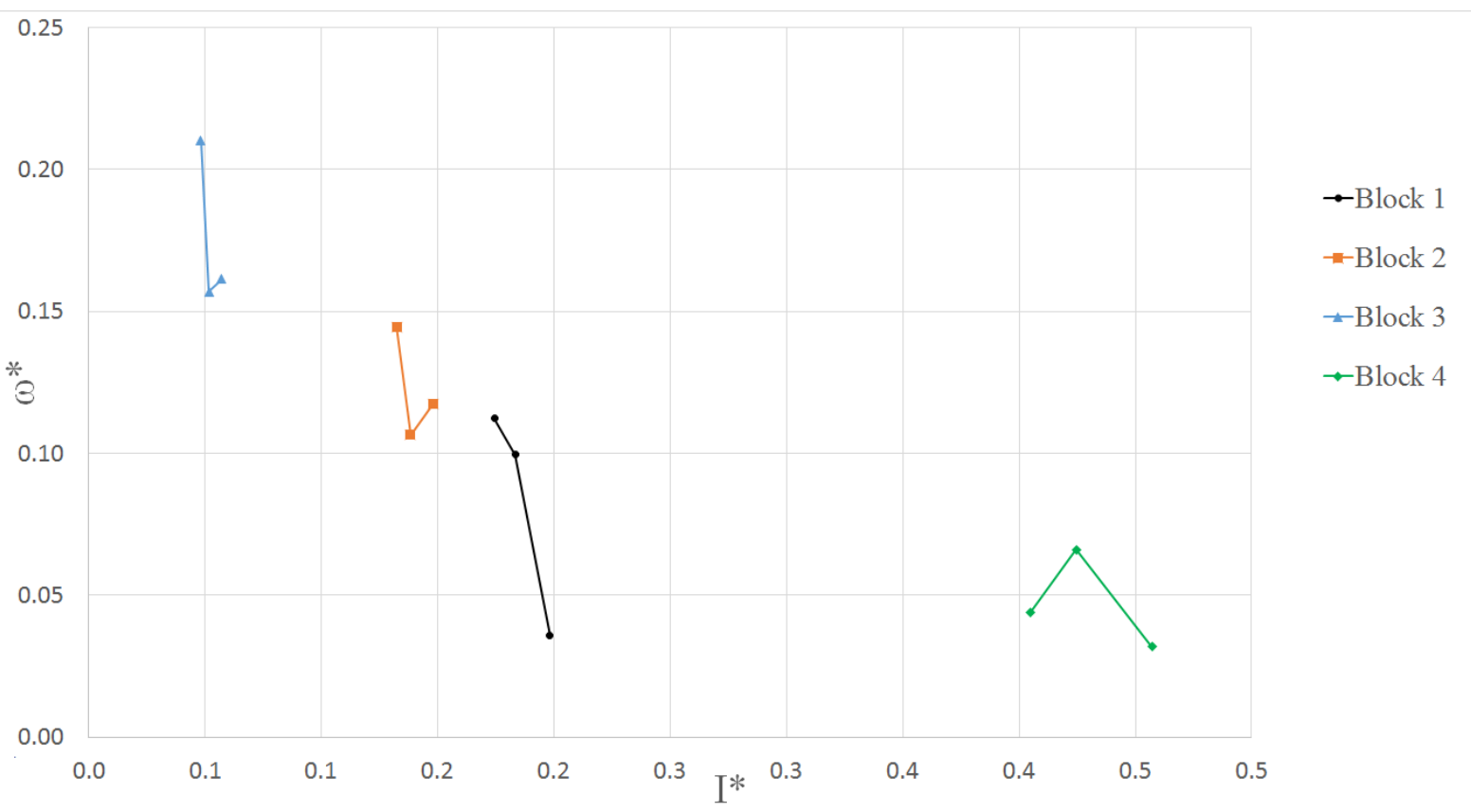

Figure 5.24: Hydraulic Diameter length scale using Equation 5-17 average velocity values. 


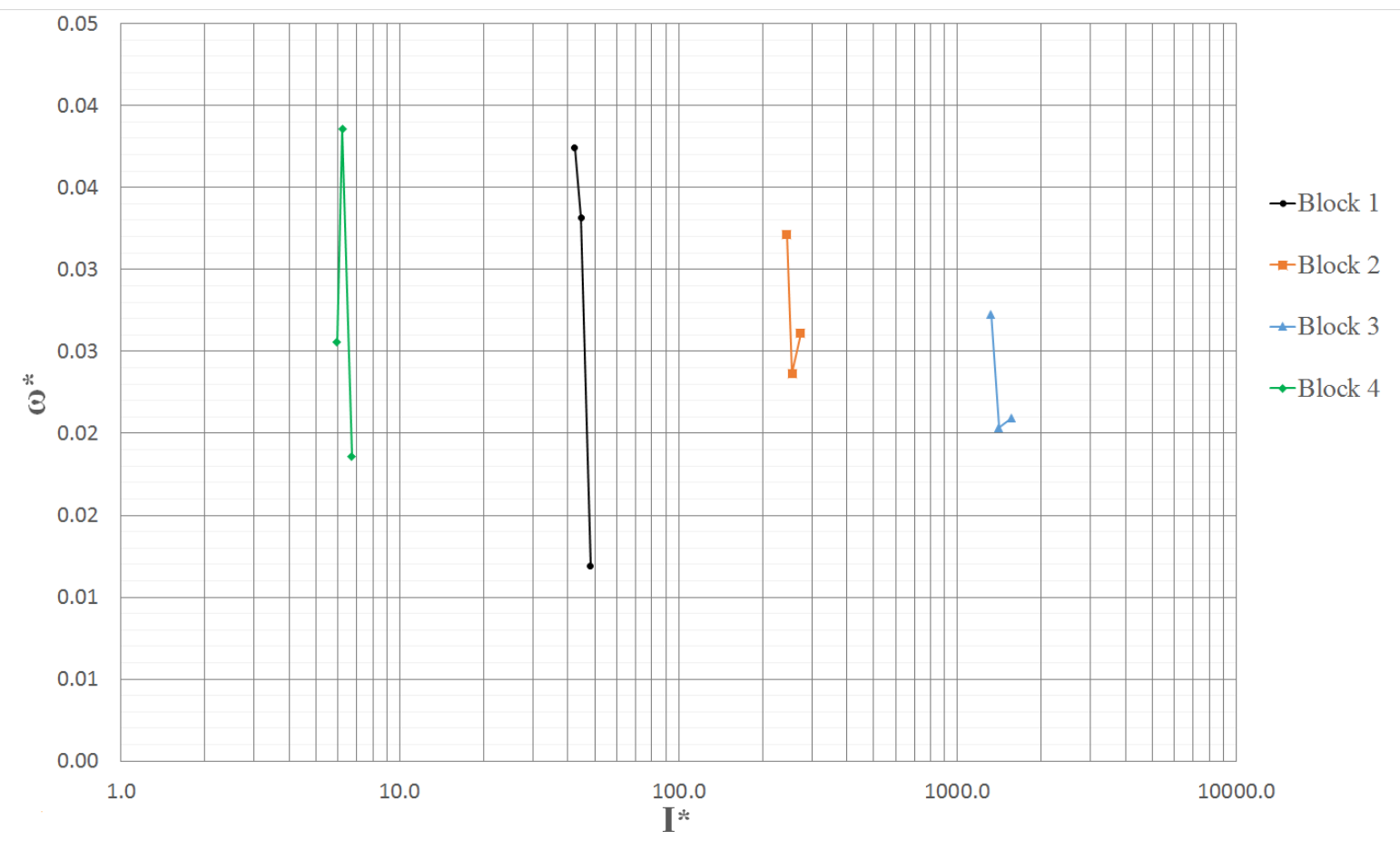

Figure 5.25: Semi-Log plot of SA/L length scale using Equation 5-17 average velocity values.

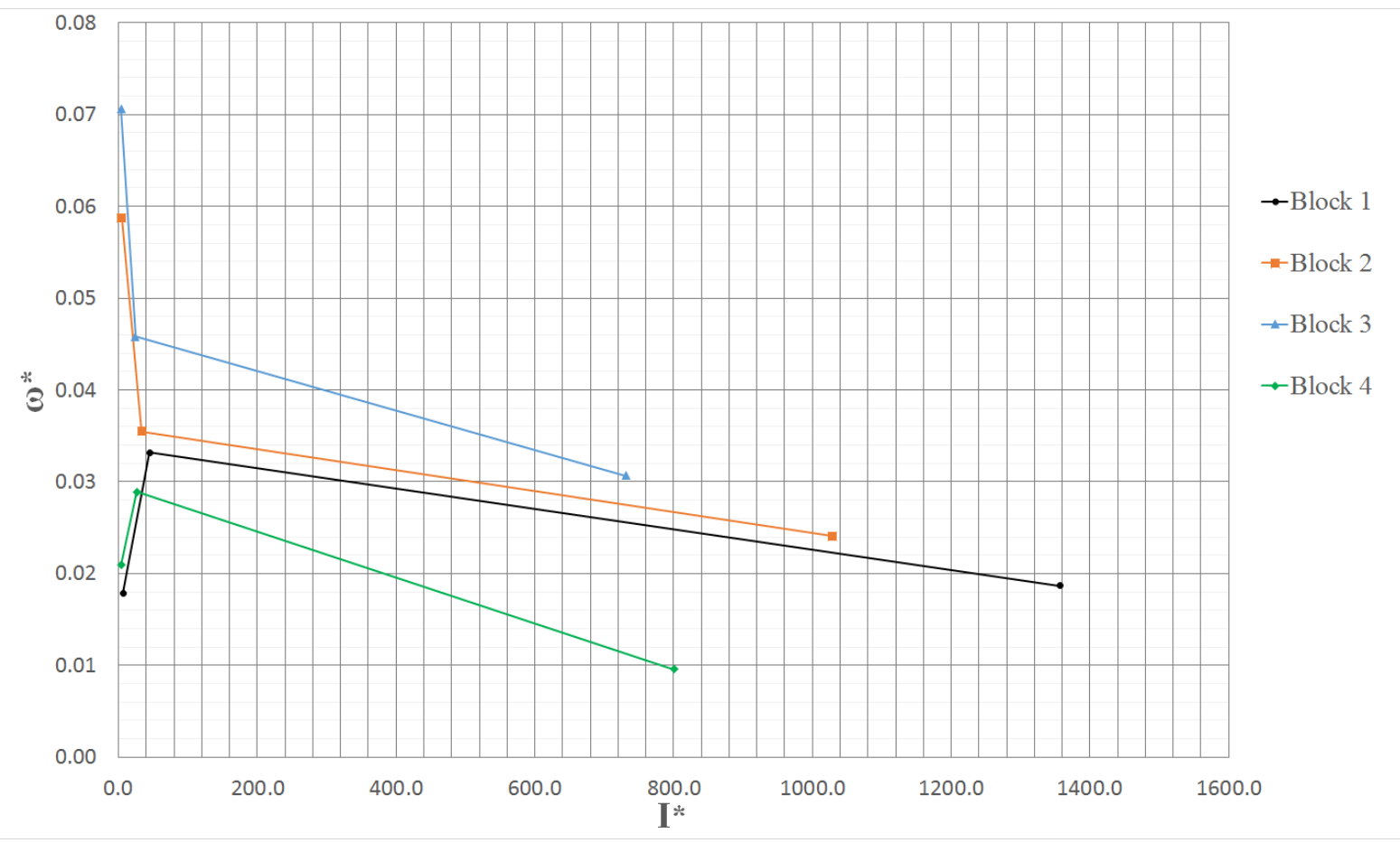

Figure 5.26: Length to bolt length scale using Equation 5-17 average velocity values. 


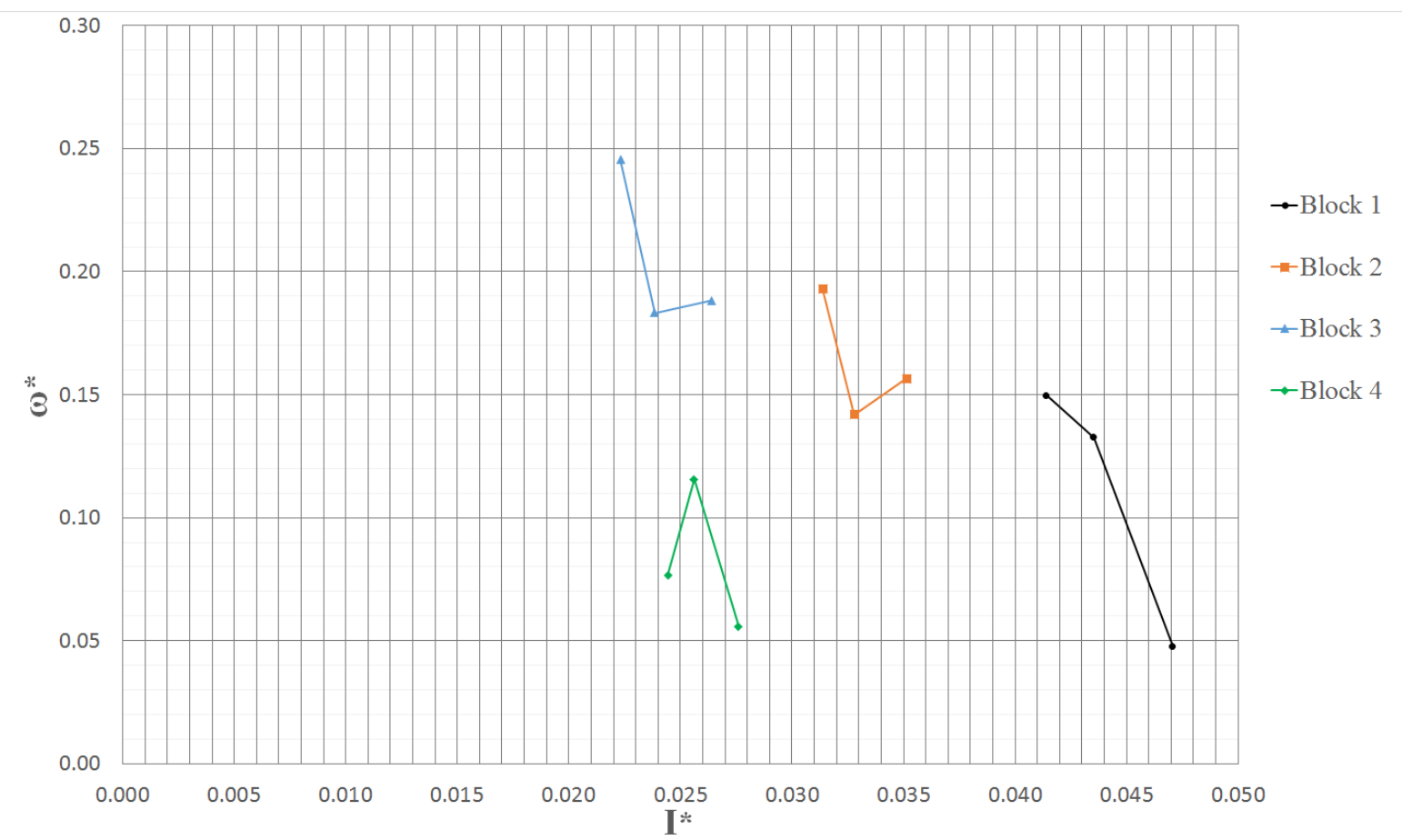

Figure 5.27: Largest dimension, W, length scale using Equation 5-17 average velocity values.

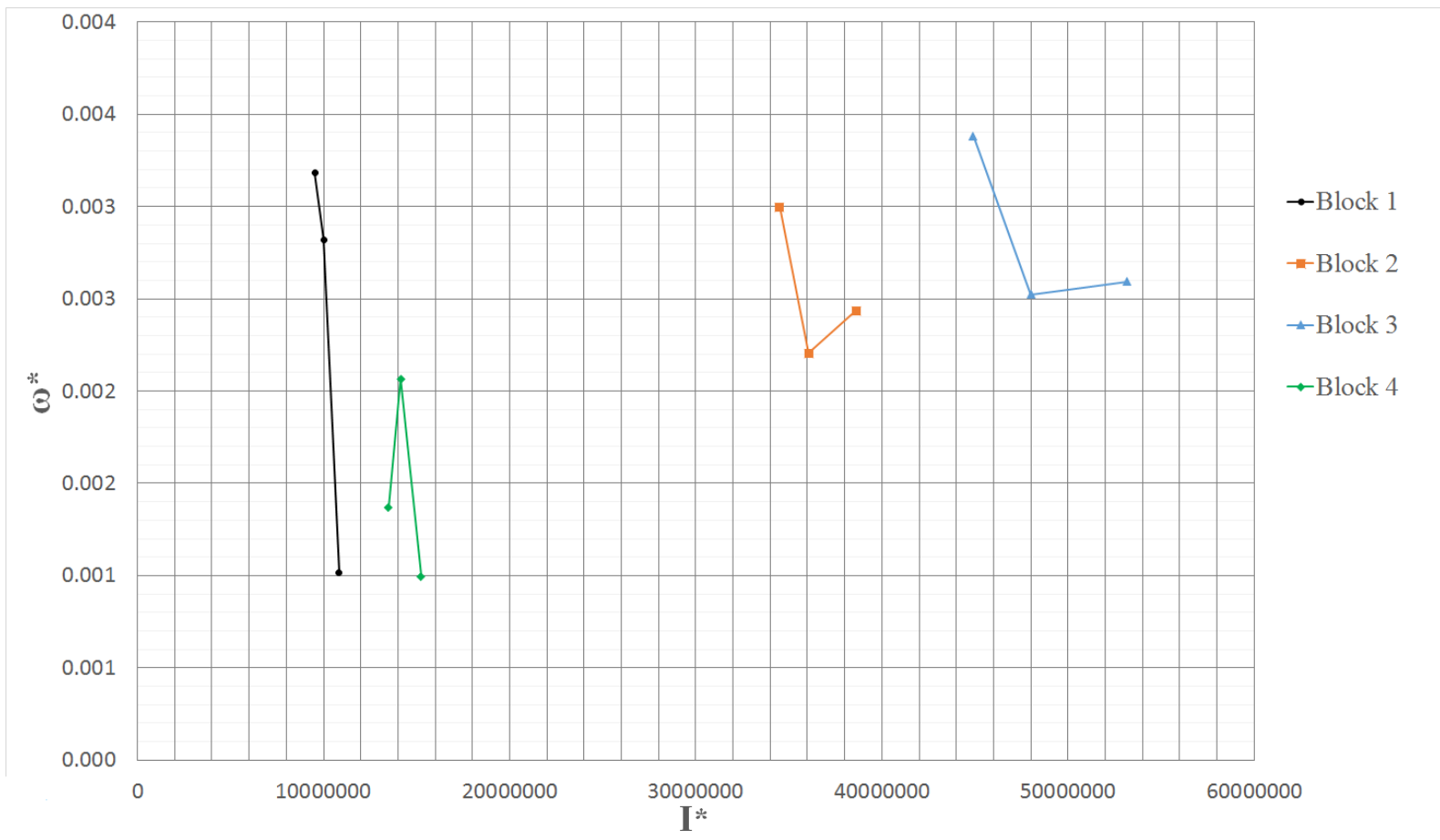

Figure 5.28: Volume/SA length scale using Equation 5-17 average velocity values. 


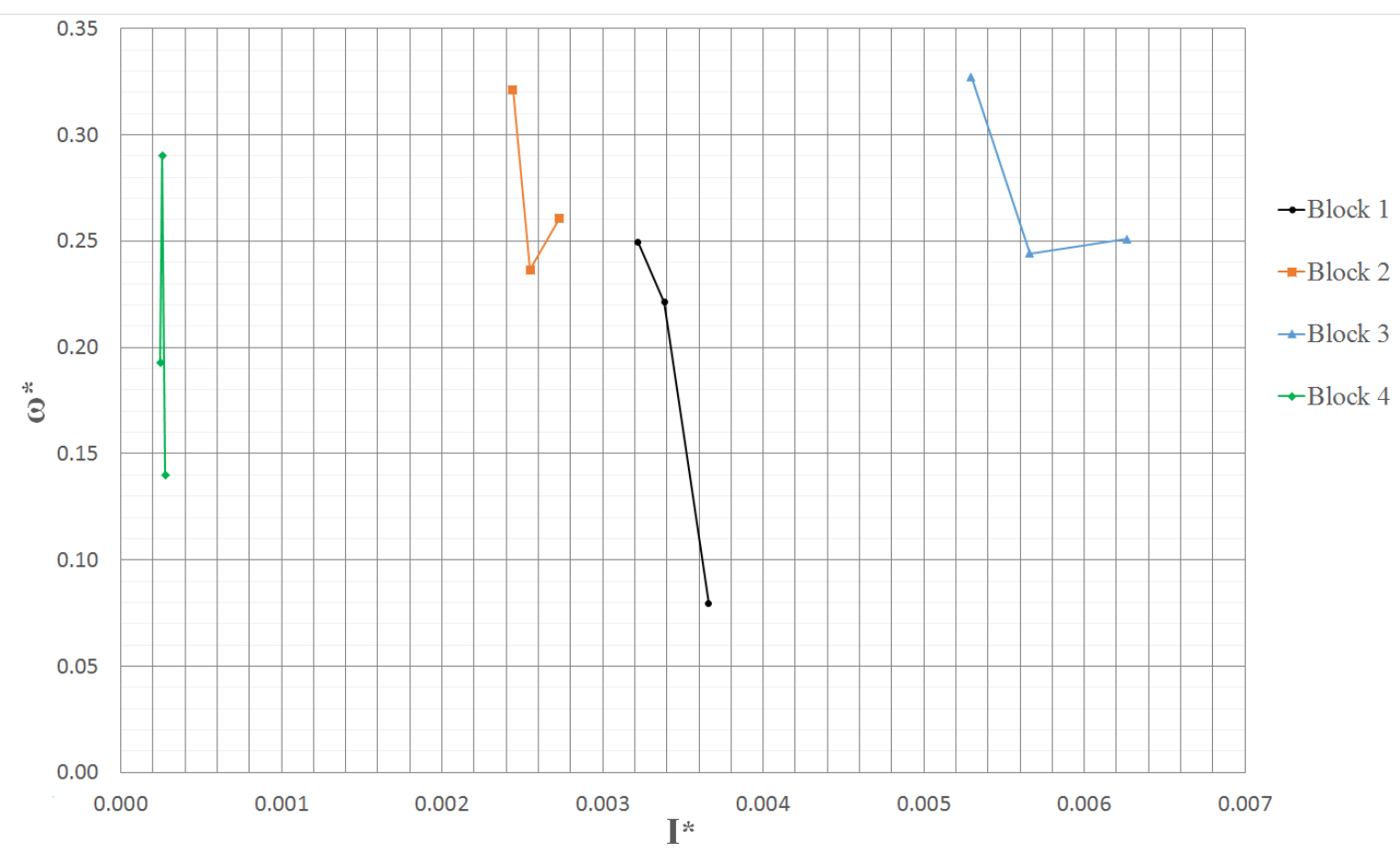

Figure 5.29: Largest dimension, W, multiplied by aspect ratio length scale using Equation 5-17 average velocity values.

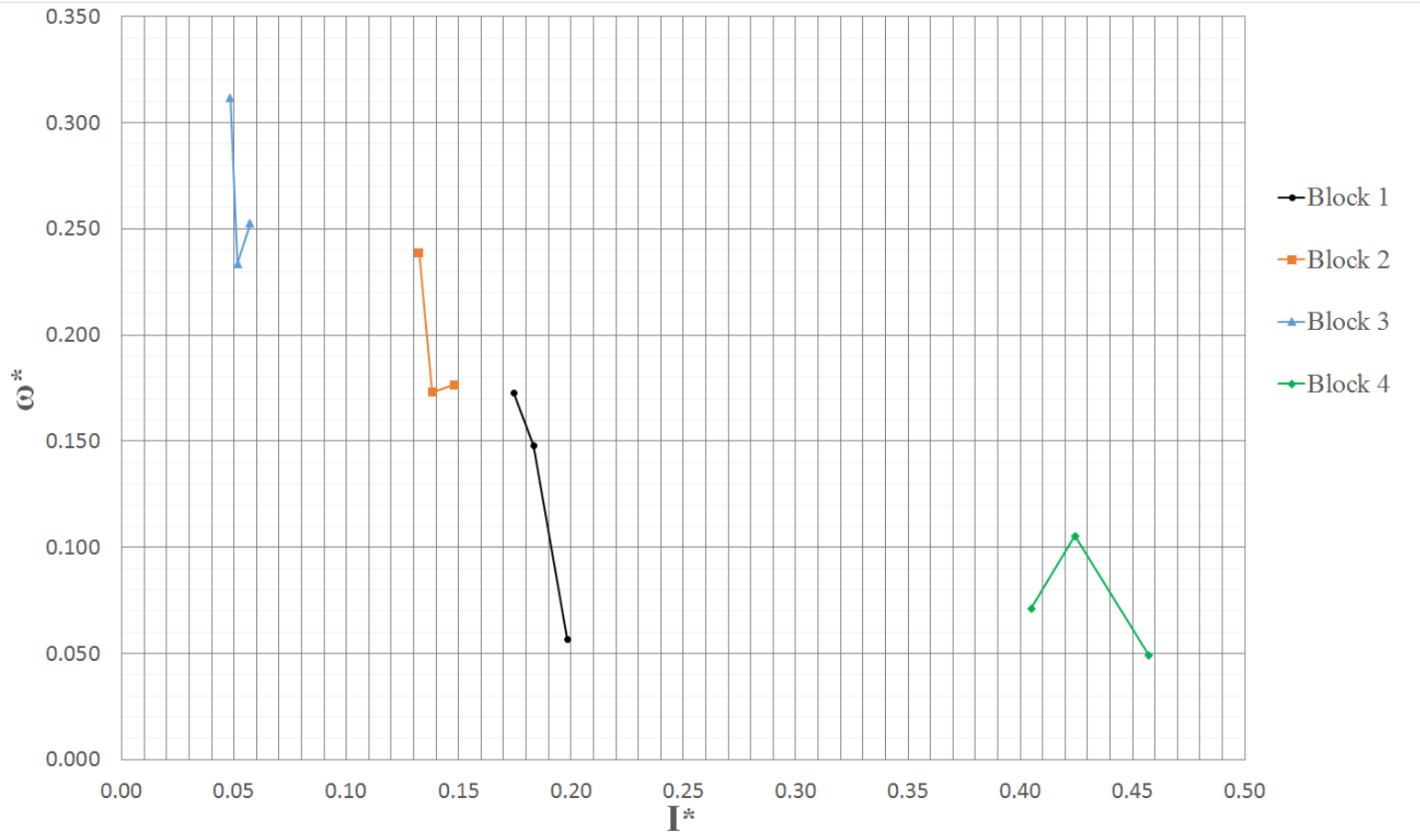

Figure 5.30: Hydraulic diameter length scale using Equation 5-18 average velocity values. 


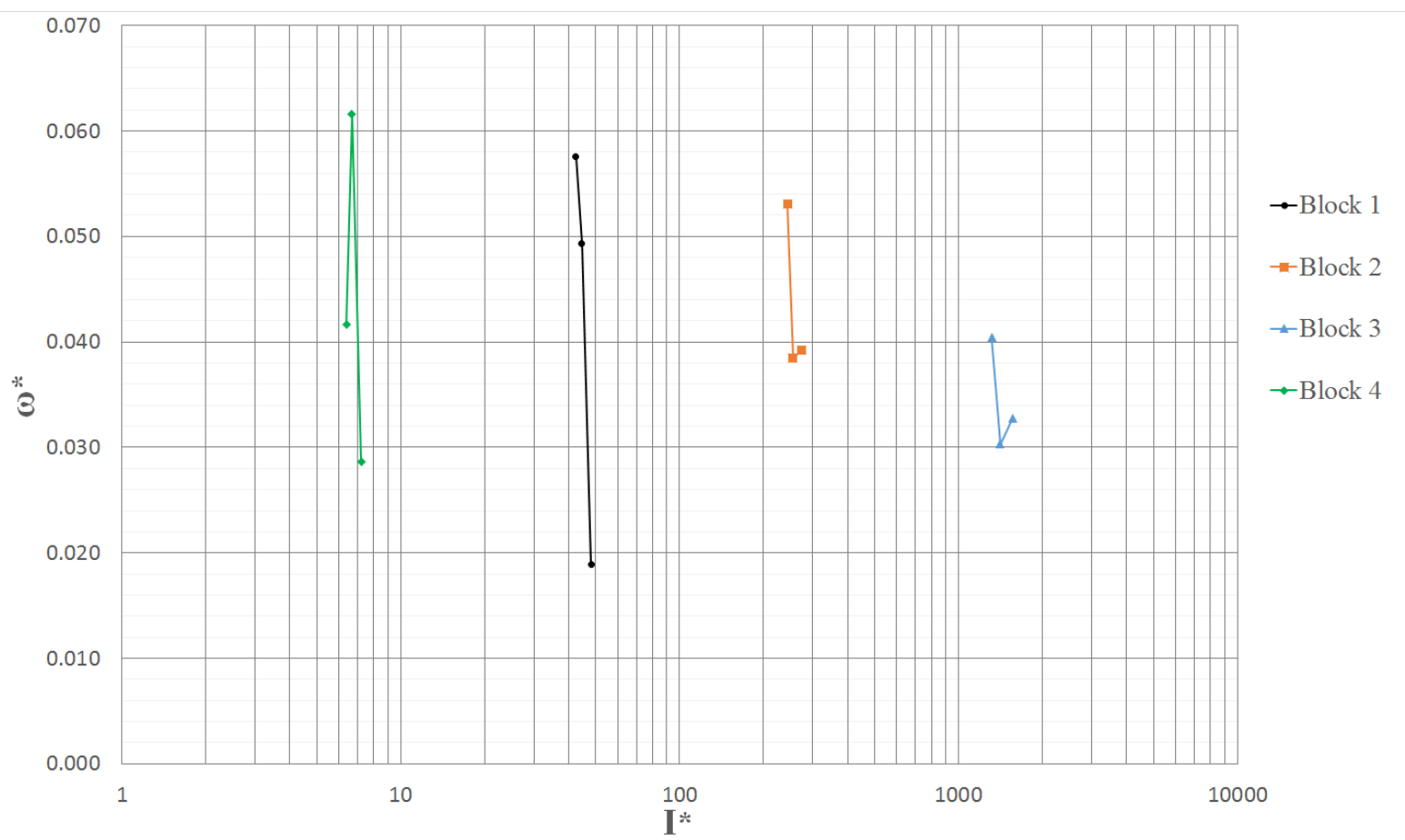

Figure 5.31:Semi-Log plot of SA/L length scale using Equation 5-18 average velocity values.

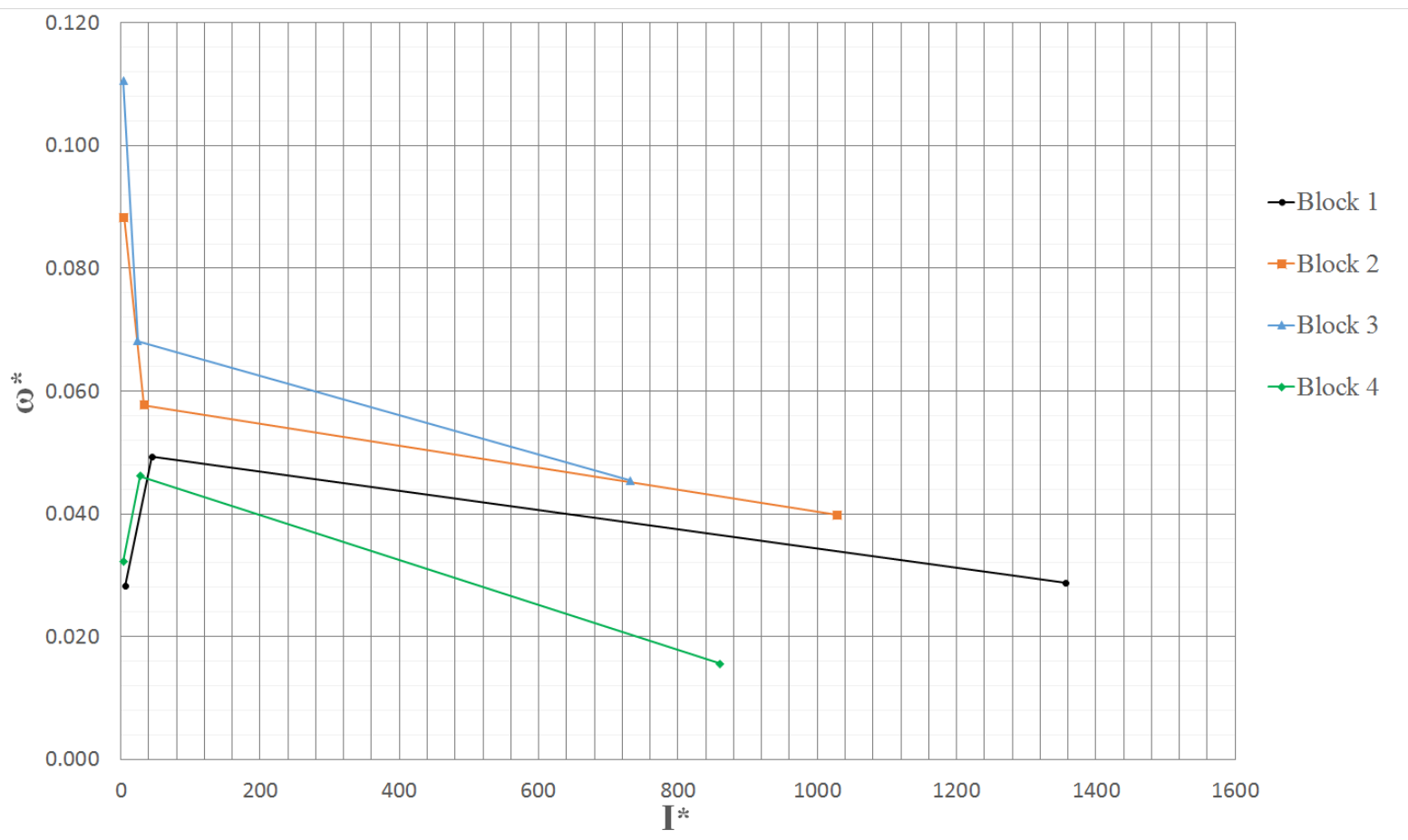

Figure 5.32: Length to bolt length scale using Equation 5-18 average velocity values. 


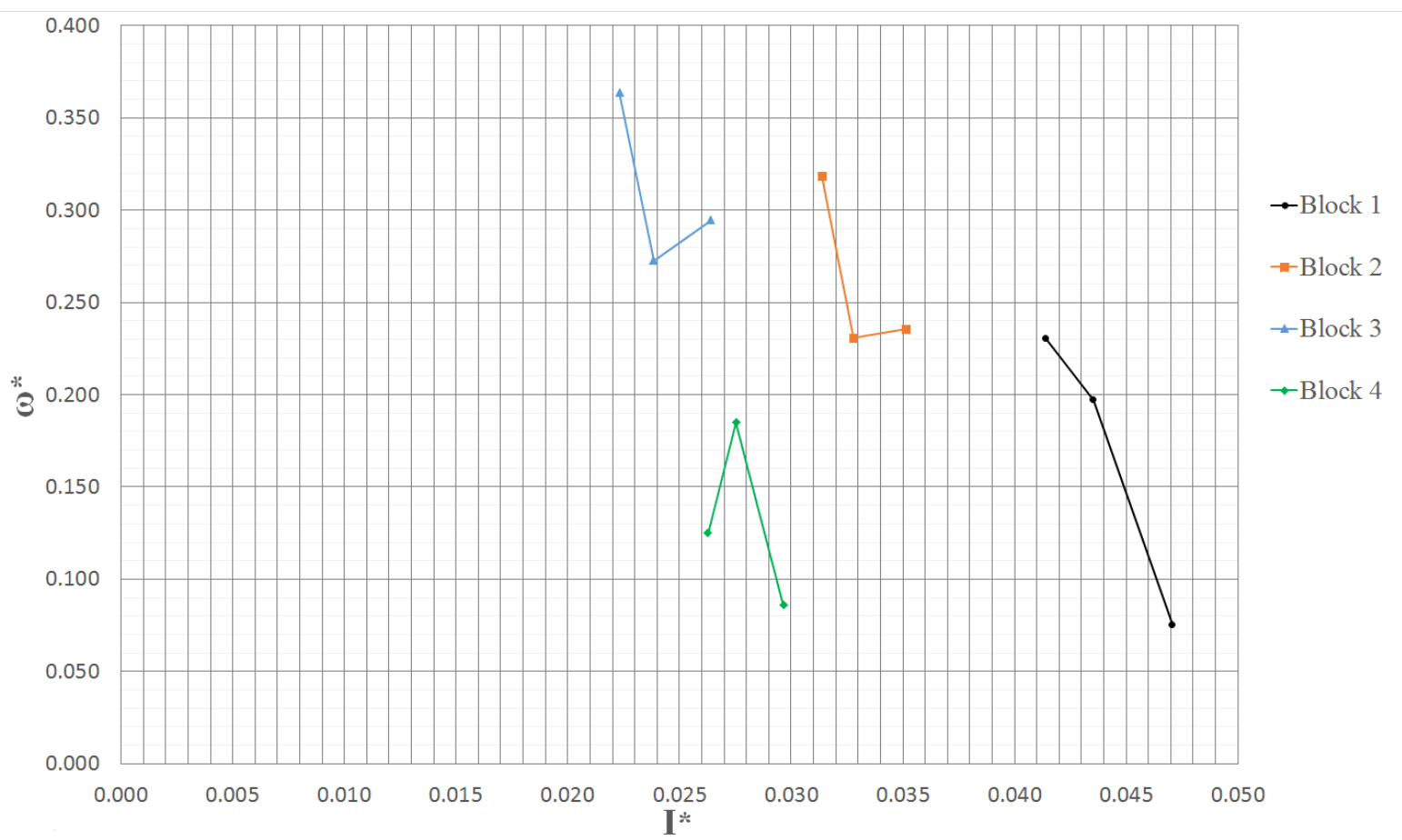

Figure 5.33: Largest dimension, W, length scale using Equation 5-18 average velocity values.

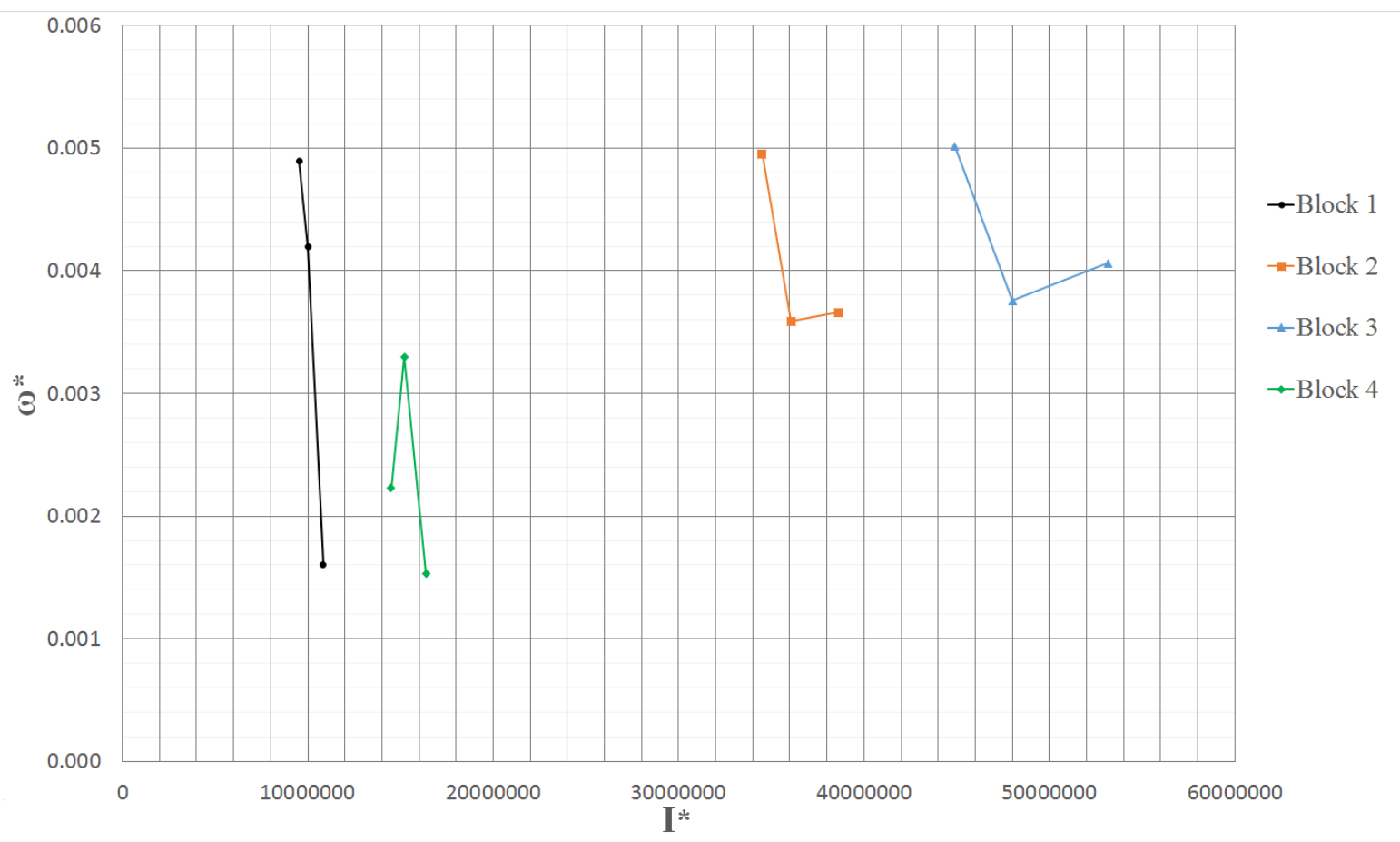

Figure 5.34: Volume/SA length scale using Equation 5-18 average velocity values. 


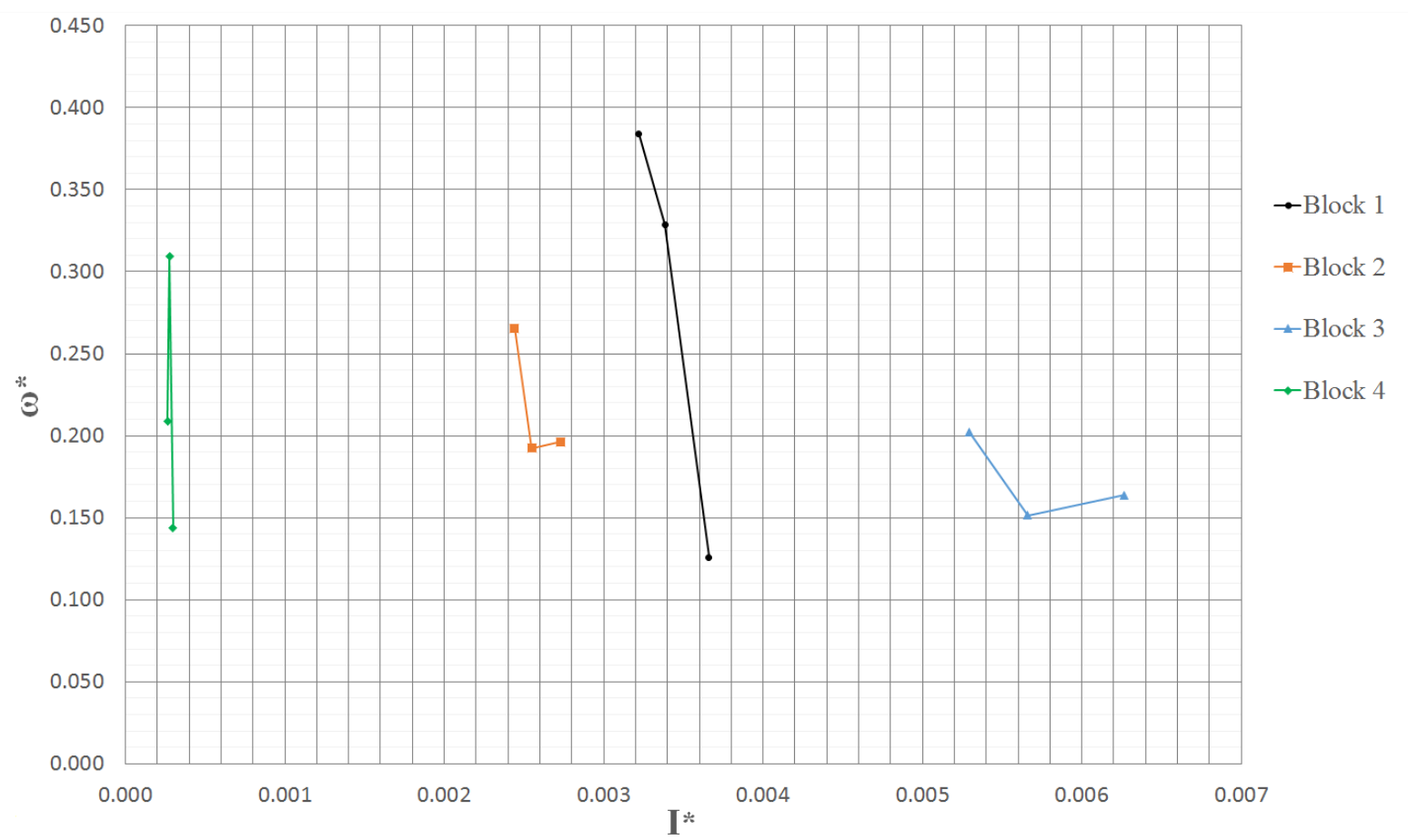

Figure 5.35: Largest dimension, W, multiplied by aspect ratio length scale using Equation 5-18 average velocity values.

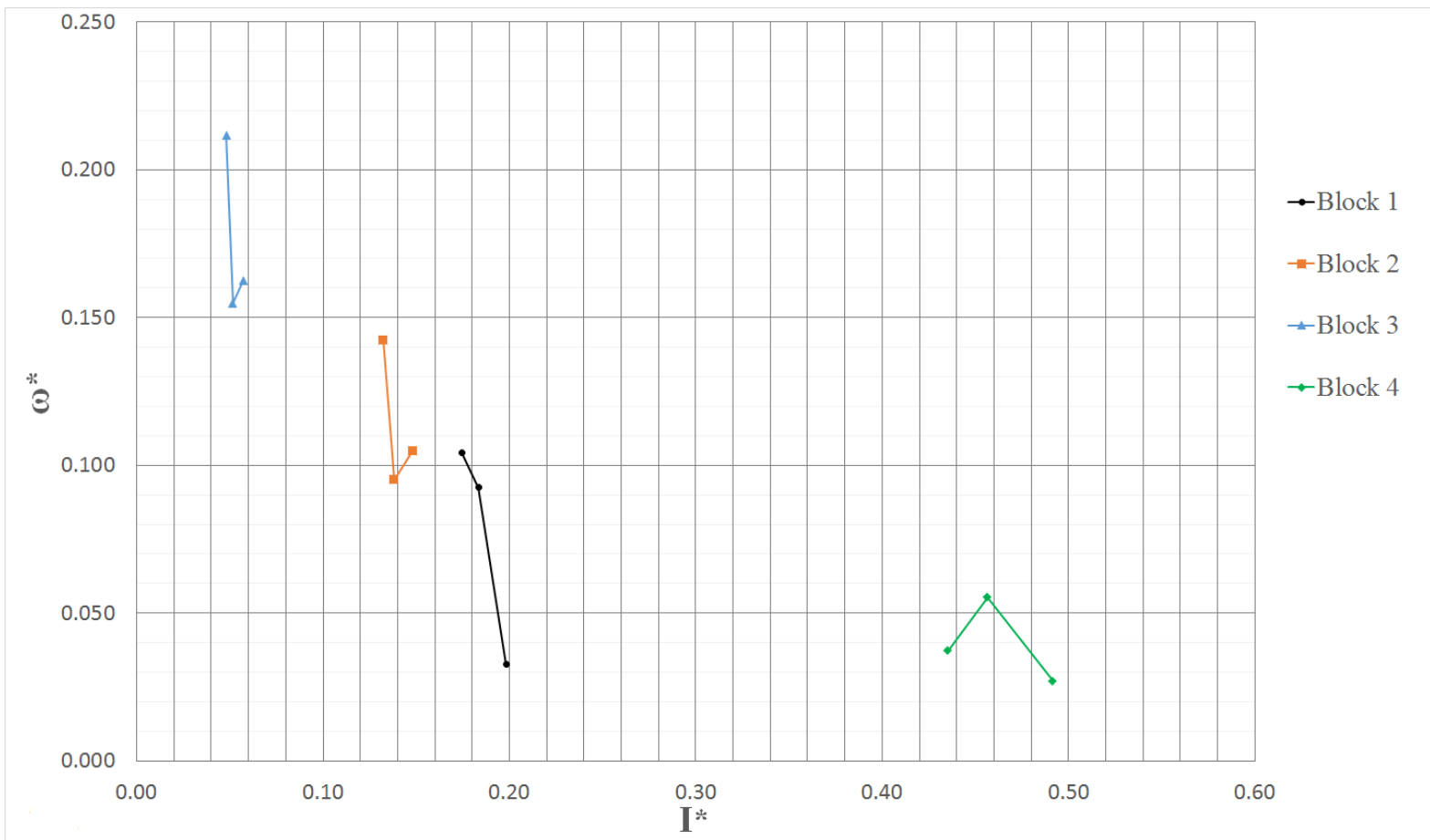

Figure 5.36: Hydraulic diameter length scale using Equation 5-19 average velocity values. 


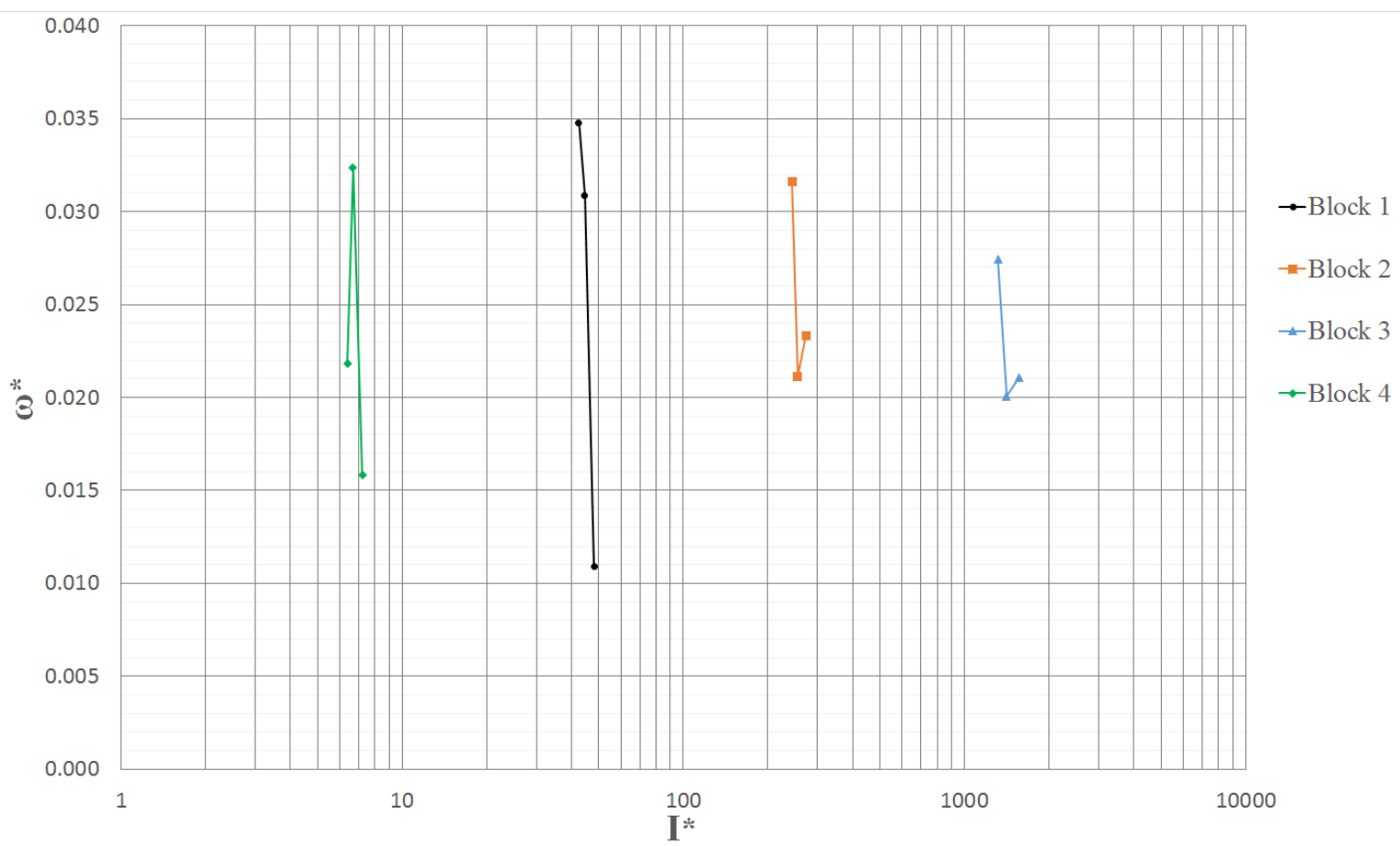

Figure 5.37: Semi-Log plot of SA/L length scale using Equation 5-19 average velocity values.

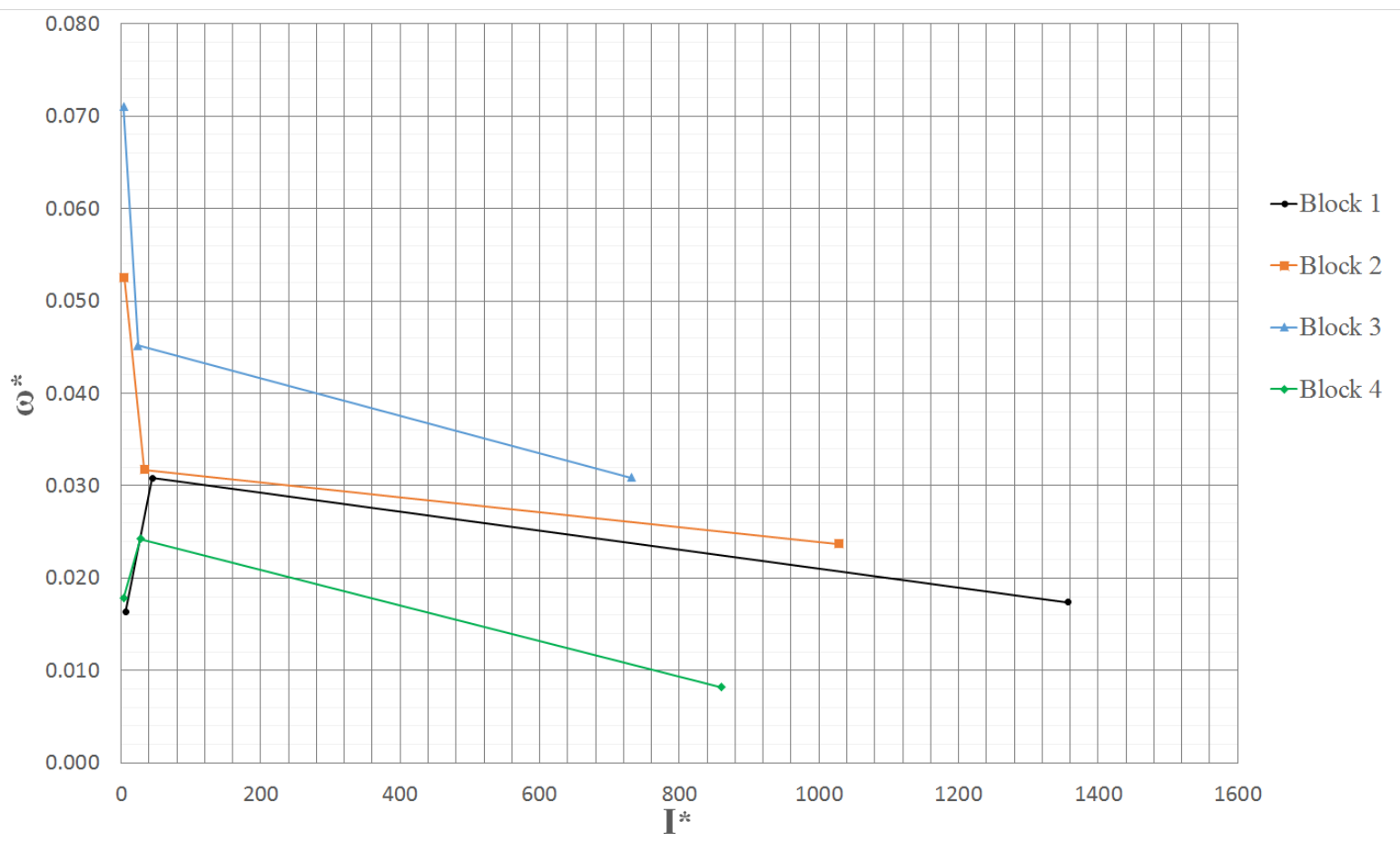

Figure 5.38: Length to bolt length scale using Equation 5-19 average velocity values. 


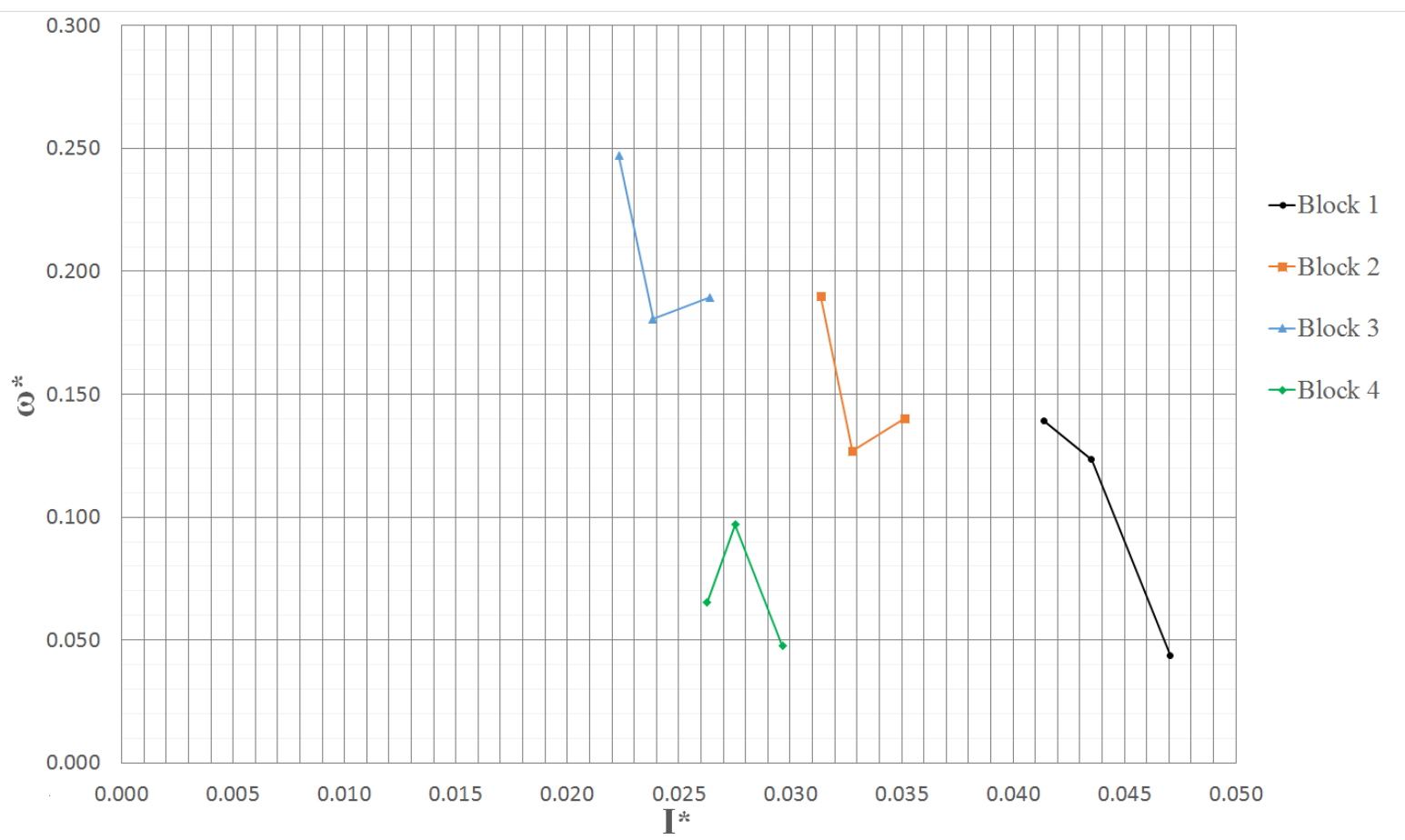

Figure 5.39: Largest dimension, W, length scale using Equation 5-19 average velocity values.

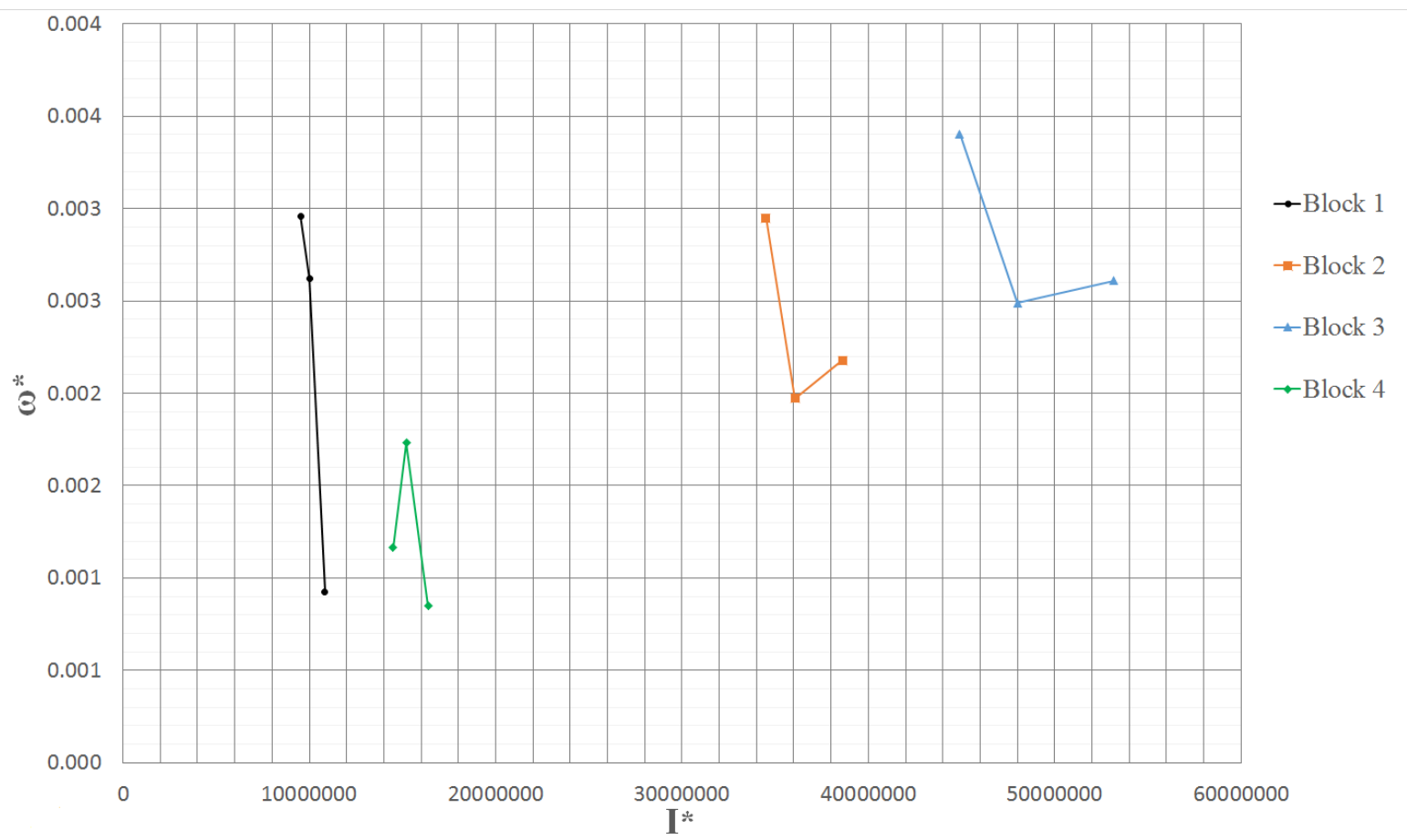

Figure 5.40: Volume/SA length scale using Equation 5-19 average velocity values. 


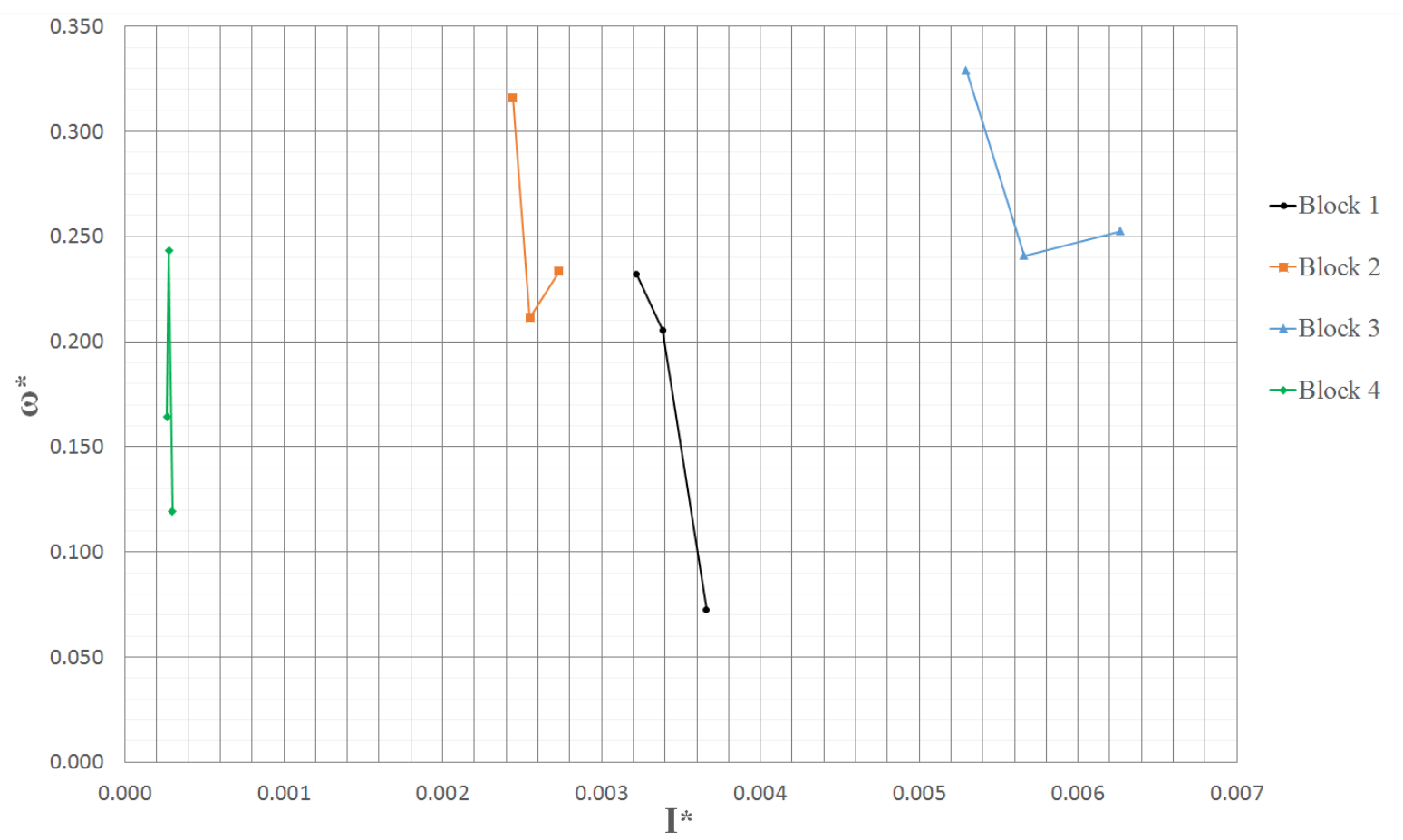

Figure 5.41: Largest dimension, W, multiplied by aspect ratio length scale using Equation 5-19 average velocity values.

From the data plotted in Figure 5.24 through Figure 5.41, the hydraulic diameter and $S A / L$ length scales seem to follow quantifiable trends. However, a reasonable assumption for a scaling relationship could not be determined from experimental data alone. A comparison to theoretical and experimental values for the length scales in question are shown in Figure 5.43 and Figure 5.44. It was assumed that other outside influences such as drag, imbalance in the blocks, and significant off-axis rotation cause the deviation from theoretical values. Theoretical velocities were calculated using Equation 5-22 and Equation 5-23.

Theoretical angular velocity was calculated using the assumption that the block rotated about the rod as a pendulum until it reached zero degrees (Figure 5.42). Points were interpolated from the three current MMOI values using the same approach as in Section 5.2. After influence from the holding rod was no longer present, the block ceased to gain rotational energy. As shown in Figure 5.42, each block started at an angle, $a$, by being supported by two pins. When one pin was quickly removed, the block rotated about the stationary pin until contact ended. Therefore, the MMOI of the block, and the starting angle, $a$, are the only factors that influenced angular velocity. The pins were placed so that they each rested one inch from the edge of the block. 


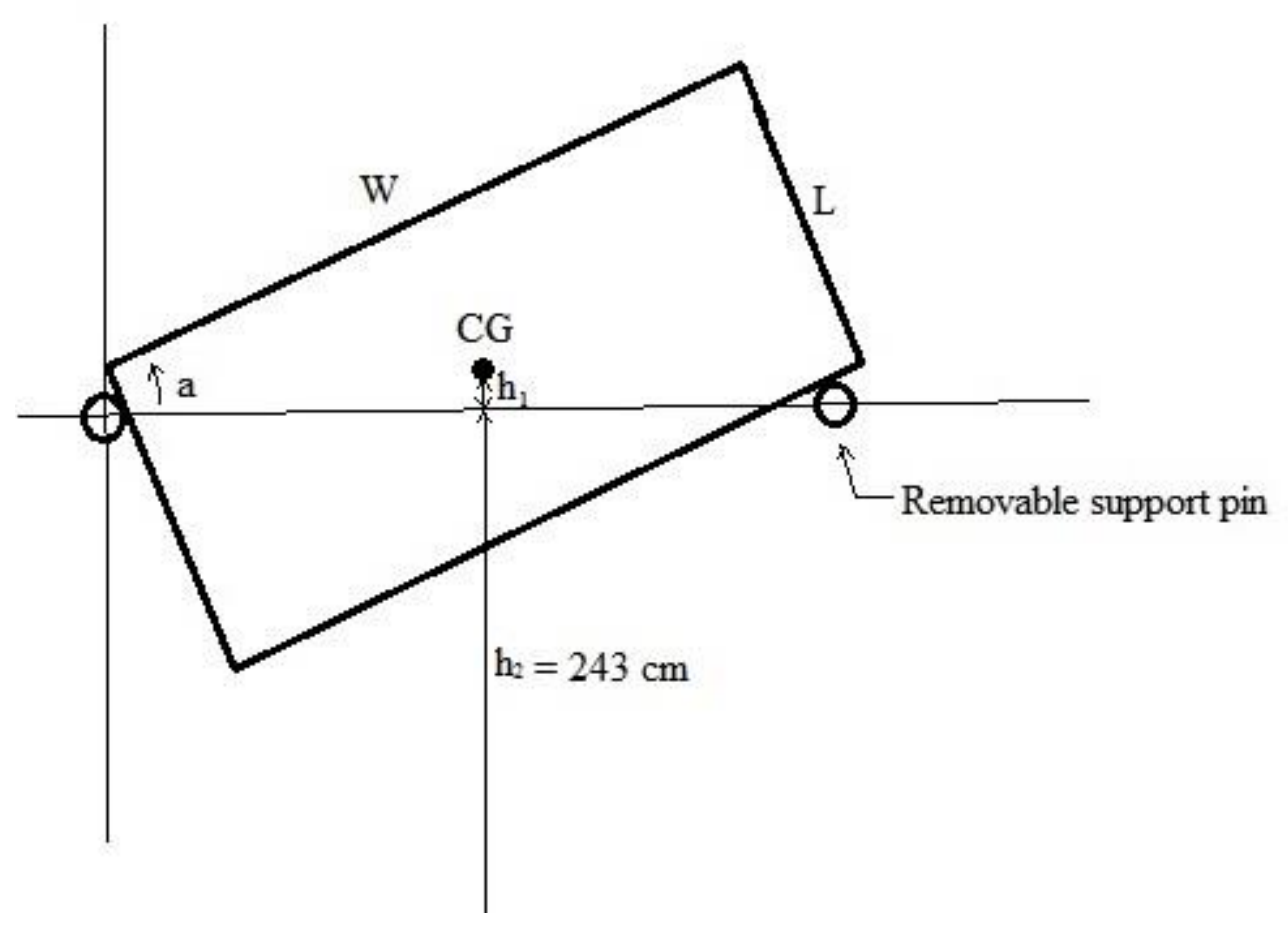

Figure 5.42: Initial condition of the blocks for drop testing.

By changing the MMOI of each block, the angular velocity was subject to change as well. Likewise, the increase or decrease in angular velocity affected the energy transformed into purely translational velocity. However, the two forms of motion were solved for independently under the assumption that the combination of translational and rotational motion was not affected by the individual effects from each velocity. Therefore, initial angular velocity (Equation 5-21) was calculated using the relationship of potential energy converting to kinetic energy in the form of rotation (Equation 5-20). Again, it was assumed that the initial rotational energy remained constant, even with the onset of translational motion when contact with the pin terminated.

$$
m_{b} g h_{1}=\frac{1}{2} I_{b} \omega_{b}^{2}
$$

Height, $h_{l}$, was determined by the distance of the center of mass of the block to the horizontal axis. The pin location was used to determine $h_{l}$ through simple geometry. Thus, the characteristics of each initial condition for the blocks were calculated and compiled in Table 5-14. 


$$
\omega_{b 1}=\sqrt{\frac{2 g m_{b}}{I_{b}}\left[\frac{W}{2}-\frac{\left(\frac{L}{2}-1\right)}{\tan (a)}\right] \sin (a)}
$$

Where $W$ and $L$ are dimensions of the block, $g$ is acceleration due to gravity, $I_{b}$ is the MMOI of the block, and $a$ is the angle of the block as it is supported by the pins. Once initial angular velocity was known, the angular velocity of the block as it disconnected from the pivot pin, $\omega_{b 2}$, was calculated.

Substituting $h_{1}$ into Equation 5-20 and rearranging the energy equations gives the equation used to determine angular velocity as it rotates about the support pin. The block's angular velocity and translational velocity as it falls into the frame of the Photron SA5 was determined using Equation 5-22 and Equation 5-23.

$$
\begin{gathered}
\omega_{b 2}=\omega_{b 1}-\frac{m_{b} R}{I_{b}}\left[\frac{\omega_{b 1} R-\sqrt{\omega_{b 1}^{2} R^{2}+2 g h_{2}\left(1+\frac{R^{2} m_{b}}{I_{b}}\right.}}{\left(1+\frac{m_{b} R^{2}}{I_{b}}\right)}\right] \\
v_{b}=\frac{\omega_{b 1} R-\sqrt{\omega_{b 1}^{2} R^{2}+2 g h_{2}\left(1+\frac{R^{2} m_{b}}{I_{b}}\right)}}{\left(1+\frac{m_{b} R^{2}}{I_{b}}\right)}
\end{gathered}
$$


Table 5-14: Block properties for theoretical calculations based on initial conditions.

\begin{tabular}{|c|c|c|c|c|c|c|c|}
\hline & I $\left[\mathrm{g}-\mathrm{cm}^{2}\right]$ & $\underset{[\mathrm{cm}]}{w}$ & $\underset{[\mathrm{cm}]}{\mathrm{L}}$ & $\begin{array}{c}a \\
\text { [deg] }\end{array}$ & $\begin{array}{c}\mathrm{h}_{1} \\
{[\mathrm{~cm}]}\end{array}$ & $\begin{array}{c}\mathbf{R} \\
{[\mathrm{cm}]}\end{array}$ & $\begin{array}{l}m_{b} \\
{[g]}\end{array}$ \\
\hline \multicolumn{8}{|l|}{ Block 1} \\
\hline MMOI 1 & 28196.7 & 25.4 & 15.2 & 30.0 & 1.9 & 14.3 & 95 \\
\hline MMOI 2 & 29642.3 & 25.4 & 15.2 & 30.0 & 1.9 & 14.3 & 95 \\
\hline MMOI 3 & 32051.6 & 25.4 & 15.2 & 30.0 & 1.9 & 14.3 & 95 \\
\hline \multicolumn{8}{|l|}{ Block 2} \\
\hline MMOI 1 & 638661.3 & 50.8 & 30.5 & 30.0 & 1.7 & 29.1 & 473 \\
\hline MMOI 2 & 667193.6 & 50.8 & 30.5 & 30.0 & 1.7 & 29.1 & 473 \\
\hline MMOI 3 & 714747.6 & 50.8 & 30.5 & 30.0 & 1.7 & 29.1 & 473 \\
\hline \multicolumn{8}{|l|}{ Block 3} \\
\hline MMOI 1 & 1124770.9 & 61.0 & 45.7 & 35.0 & 0.8 & 37.5 & 848 \\
\hline MMOI 2 & 1202314 & 61.0 & 45.7 & 35.0 & 0.8 & 37.5 & 848 \\
\hline MMOI 3 & 1331552.5 & 61.0 & 45.7 & 35.0 & 0.8 & 37.5 & 848 \\
\hline \multicolumn{8}{|l|}{ Block 4} \\
\hline MMOI 1 & 117487.4 & 38.0 & 15.3 & 20 & 1.7 & 20.1 & 164 \\
\hline MMOI 2 & 123187.4 & 38.0 & 15.3 & 20 & 1.7 & 20.1 & 164 \\
\hline MMOI 3 & 132694.5 & 38.0 & 15.3 & 20 & 1.7 & 20.1 & 164 \\
\hline
\end{tabular}

Table 5-15 shows the velocity data obtained using the tracker software, as well as properties of the block used to calculate dimensionless terms. The measured values were compared to theoretical values in Figure 5.43 and Figure 5.44. The hydraulic diameter length scale analyzed in Figure 5.43 follows a similar trend to the theoretical data. Additionally, the SA/L length scale used in Figure 5.44 also follows the theatrical data. The hydraulic diameter length scale was not considered since the trend was not as determined for the other experiments. Block 2 and Block 3 in Figure 5.44 seem to follow the same trend as the theoretical data, but the blocks have a significantly higher dimensionless angular velocity. This could be due to an inaccurate representation of the translational velocity or the relatively high value of the length scale compared to the slow velocity. 
Table 5-15: Tracker data and additional properties used for dimensionless term calculations.

\begin{tabular}{|c|c|c|c|c|c|c|}
\hline Block 1 & $\mathrm{I}\left(\mathrm{g}-\mathrm{cm}^{2}\right)$ & $\begin{array}{c}\mathrm{U}_{\mathrm{X} \text { _avg }} \\
(\mathrm{cm} / \mathrm{sec})\end{array}$ & $\begin{array}{c}\omega_{\text {avg }} \\
(\mathrm{rad} / \mathrm{sec})\end{array}$ & $\begin{array}{l}\mathrm{L} \text { to bolt } \\
{[\mathrm{cm}]}\end{array}$ & $\mathrm{W}(\mathrm{cm})$ & 25.4 \\
\hline MMOI 1 & 28196.7 & 322.8 & 2.5 & 3.2 & time (sec) & 0.2 \\
\hline MMOI 2 & 29642.3 & 320.2 & 2.3 & 6.4 & $\rho\left(\mathrm{g} / \mathrm{cm}^{3}\right)$ & $6.44 \mathrm{E}-02$ \\
\hline MMOI 3 & 32051.6 & 305.4 & 0.9 & 9.5 & $\mathrm{~g}\left(\mathrm{~cm} / \mathrm{sec}^{2}\right)$ & 981 \\
\hline Block 2 & $\mathrm{I}\left(\mathrm{g}-\mathrm{cm}^{2}\right)$ & $\begin{array}{c}\mathrm{u}_{\mathrm{X} \text { _avg }} \\
(\mathrm{cm} / \mathrm{sec})\end{array}$ & $\begin{array}{c}\omega_{\text {avg }} \\
(\mathrm{rad} / \mathrm{sec})\end{array}$ & $\begin{array}{l}\mathrm{L} \text { to bolt } \\
{[\mathrm{cm}]}\end{array}$ & $\mathrm{W}(\mathrm{cm})$ & 50.8 \\
\hline MMOI 1 & 638661.3 & 345.0 & 1.8 & 6.4 & time (sec) & 0.2 \\
\hline MMOI 2 & 667193.6 & 327.1 & 1.2 & 12.7 & $\rho\left(\mathrm{g} / \mathrm{cm}^{3}\right)$ & $6.01 \mathrm{E}-02$ \\
\hline MMOI 3 & 714747.6 & 343.1 & 1.4 & 19.1 & $\mathrm{~g}\left(\mathrm{~cm} / \mathrm{sec}^{2}\right)$ & 981 \\
\hline Block 3 & $\mathrm{I}\left(\mathrm{g}-\mathrm{cm}^{2}\right)$ & $\begin{array}{c}\mathrm{u}_{\mathrm{X}} \mathrm{avg} \\
(\mathrm{cm} / \mathrm{sec})\end{array}$ & $\begin{array}{c}\omega_{\mathrm{avg}} \\
(\mathrm{rad} / \mathrm{sec})\end{array}$ & $\begin{array}{l}\mathrm{L} \text { to bolt } \\
{[\mathrm{cm}]}\end{array}$ & $\mathrm{W}(\mathrm{cm})$ & 61.0 \\
\hline MMOI 1 & 1124770.9 & 370.5 & 2.0 & 7.6 & time (sec) & 0.2 \\
\hline MMOI 2 & 1202314 & 360.8 & 1.4 & 15.2 & $\rho\left(\mathrm{g} / \mathrm{cm}^{3}\right)$ & 5.99E-02 \\
\hline MMOI 3 & 1331552.5 & 358.3 & 1.6 & 22.9 & $\mathrm{~g}\left(\mathrm{~cm} / \mathrm{sec}^{2}\right)$ & 981 \\
\hline Block 4 & $\mathrm{I}\left(\mathrm{g}-\mathrm{cm}^{2}\right)$ & $\begin{array}{c}\mathrm{u}_{\mathrm{X} \_\mathrm{avg}} \\
(\mathrm{cm} / \mathrm{sec})\end{array}$ & $\begin{array}{c}\omega_{\mathrm{avg}} \\
(\mathrm{rad} / \mathrm{sec})\end{array}$ & $\begin{array}{l}\mathrm{L} \text { to bolt } \\
{[\mathrm{cm}]}\end{array}$ & $\mathrm{W}(\mathrm{cm})$ & 38.1 \\
\hline MMOI 1 & 117487.4 & 303.8 & 0.7 & 4.8 & time $(\mathrm{sec})$ & 0.2 \\
\hline MMOI 2 & 123187.4 & 306.7 & 1.1 & 9.5 & $\rho\left(\mathrm{g} / \mathrm{cm}^{3}\right)$ & $5.57 \mathrm{E}-02$ \\
\hline MMOI 3 & 132694.5 & 311.8 & 0.3 & 14.3 & $\mathrm{~g}\left(\mathrm{~cm} / \mathrm{sec}^{2}\right)$ & 981 \\
\hline
\end{tabular}

From the results of the plotted dimensionless terms, it was assumed that using Equation 5-18 to calculate velocity was the most appropriate choice. Therefore, for the drop testing data, plots and table data was based on this method of velocity calculation. Similar to the horizontal table test, the length scale $S A / L$ was used to further evaluate scaling relationships between dimensionless terms. 


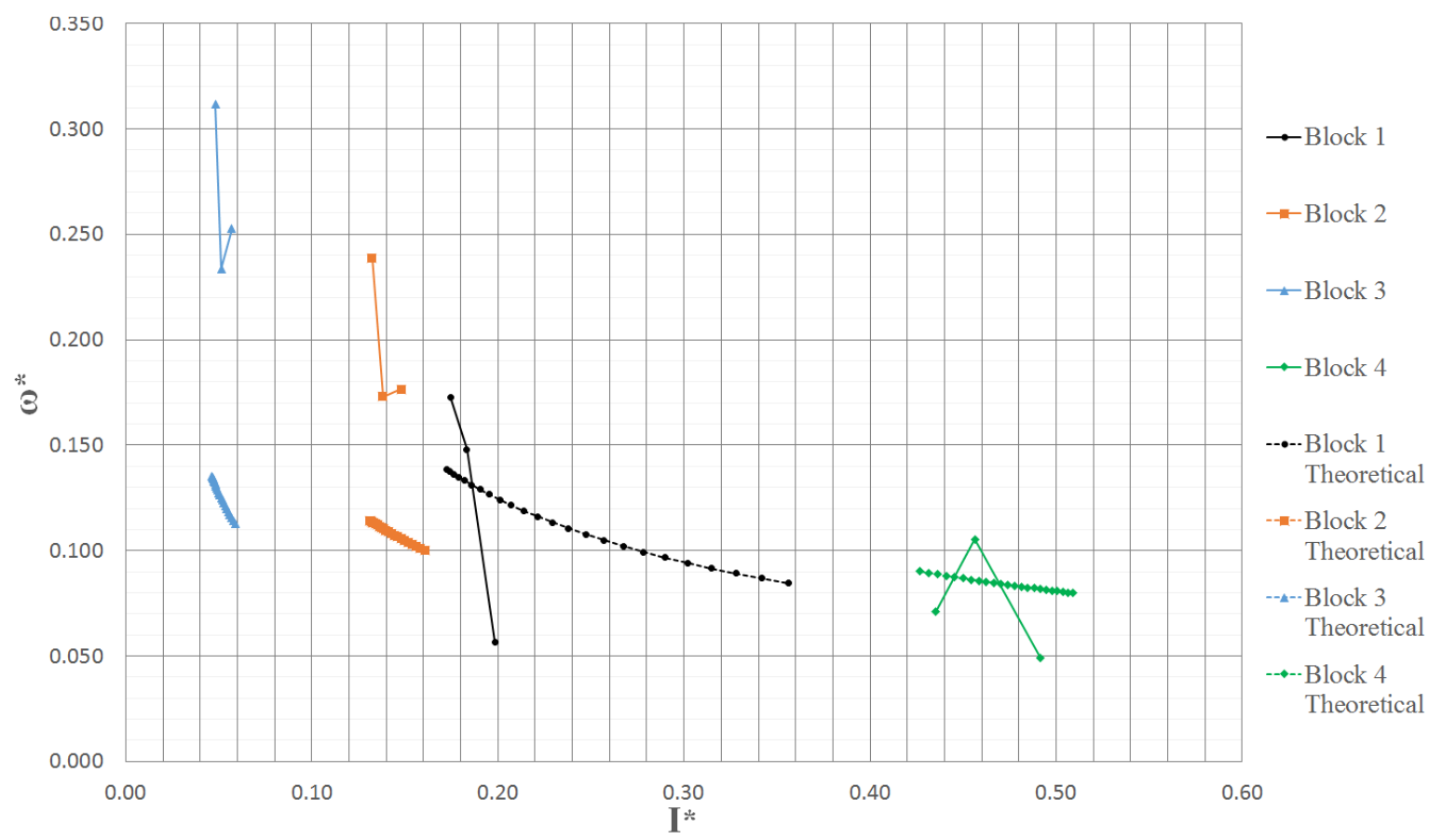

Figure 5.43: Experimental dimensionless terms versus theoretical dimensionless terms for the length scale of hydraulic diameter, using the velocity calculations based off of Equation 5-18.

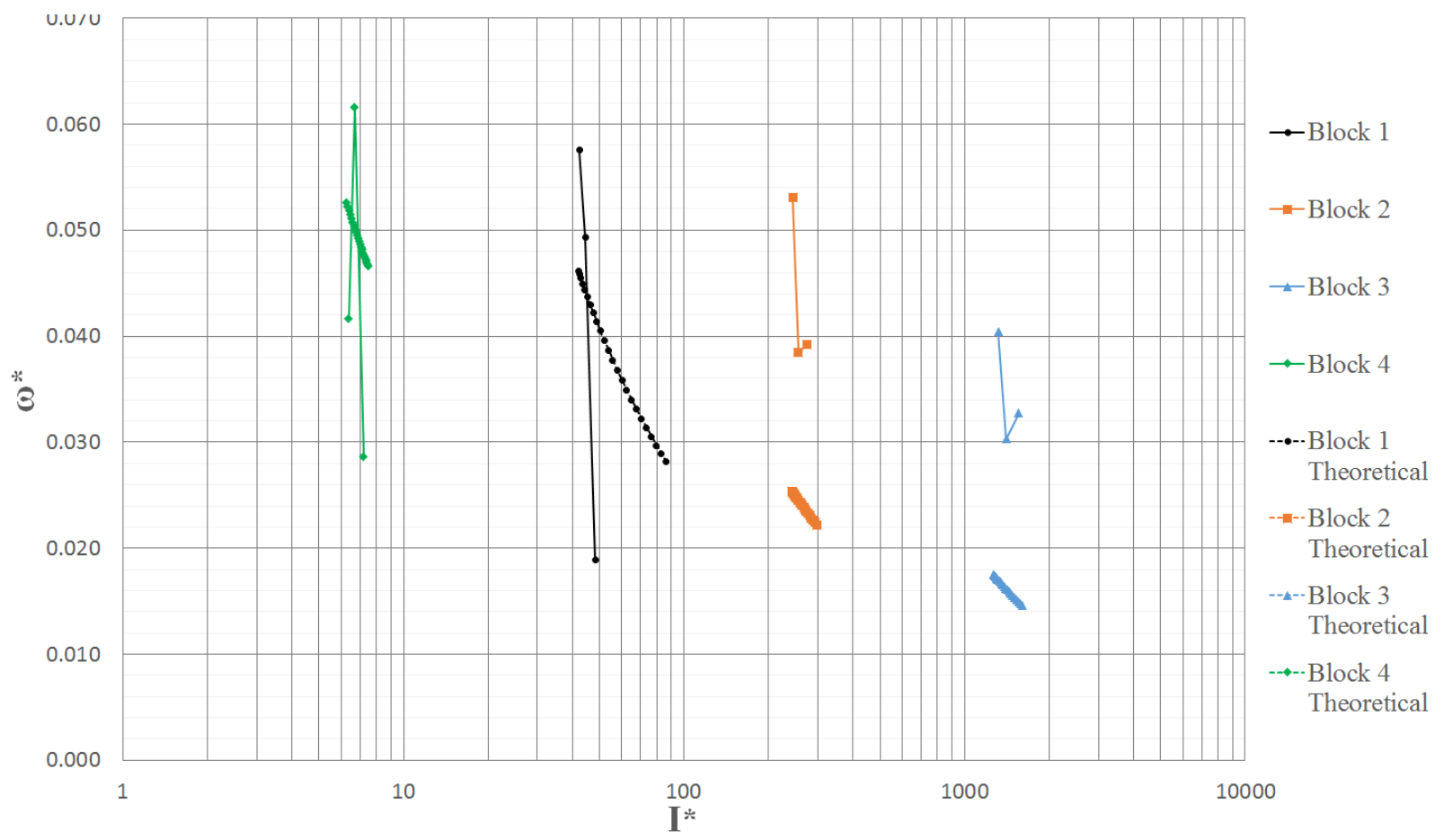

Figure 5.44: Experimental dimensionless terms versus theoretical dimensionless terms for the length scale of $S A / L$, using the velocity calculations based off of Equation 5-18. 
Experimental data was similar to theoretical, but assumptions made caused the data to be slightly skewed. An ANOVA analysis performed on the data showed more detail into what factors influence translational velocity, angular velocity, and landing location.

Table 5-16: Drop test block velocities collected for ANOVA analysis.

\begin{tabular}{|c|c|c|c|c|}
\hline \multirow{4}{*}{} & \multicolumn{4}{|c|}{$\mathbf{U}_{\mathbf{x}}$ [cm/sec] } \\
\cline { 2 - 5 } & \multicolumn{4}{|c|}{ Size } \\
\cline { 2 - 5 } & Block 1 & Block 2 & Block 3 & Block 4 \\
\hline \multirow{3}{*}{ MMOI 1 } & 325 & 343 & 374 & 305 \\
\cline { 2 - 5 } & 313 & 352 & 367 & 311 \\
\cline { 2 - 5 } & 331 & 341 & 370 & 295 \\
\cline { 2 - 5 } & 322 & 337 & 366 & 292 \\
\cline { 2 - 6 } & 318 & 317 & 358 & 307 \\
\hline \multirow{3}{*}{ MMOI 2 } & 299 & 343 & 358 & 307 \\
\cline { 2 - 6 } & 322 & 335 & 355 & 301 \\
\cline { 2 - 6 } & 296 & 352 & 364 & 327 \\
\hline
\end{tabular}

Table 5-17: ANOVA calculations of MMOI and size effects on translational velocity

\begin{tabular}{|ccccccc|}
\hline $\begin{array}{c}\text { Source of } \\
\text { Variation }\end{array}$ & SS & $d f$ & MS & $F$ & P-value & F crit \\
\hline Sample & 332.2 & 2 & 166.1 & 1.9 & $1.7 \mathrm{E}-01$ & 3.4 \\
Columns & 16749.1 & 3 & 5583.0 & 65.0 & $1.1 \mathrm{E}-11$ & 3.0 \\
Interaction & 1129.4 & 6 & 188.2 & 2.2 & $7.9 \mathrm{E}-02$ & 2.5 \\
Within & 2061.3 & 24 & 85.9 & & & \\
Total & 20272.0 & 35 & & & & \\
\hline
\end{tabular}

From data in Table 5-17, the P-value for Samples indicates no interaction. This is determined one or both of two visual assessments: $F<F$ crit and $P$-value $>$ alpha. The Columns section, however, has significant interaction. Thus, the null hypothesis was rejected for this section. There was some influence by interaction between the factors, but the size of the block, represented by Columns, was the main influencing factor on translational velocity. 
Table 5-18: Drop test block angular velocities collected for ANOVA analysis.

\begin{tabular}{|c|c|c|c|c|}
\hline \multirow{4}{*}{} & \multicolumn{4}{|c|}{$\boldsymbol{\omega}$ [rad/sec] } \\
\cline { 2 - 5 } & \multicolumn{4}{|c|}{ Size } \\
\cline { 2 - 5 } & Block 1 & Block 2 & Block 3 & Block 4 \\
\hline \multirow{3}{*}{ MMOI 1 } & 2.4 & 1.7 & 2.0 & 0.5 \\
\cline { 2 - 5 } & 2.3 & 1.8 & 2.0 & 1.2 \\
\cline { 2 - 5 } & 2.5 & 2.0 & 2.0 & 0.8 \\
\hline \multirow{3}{*}{ MMOI 2 } & 1.7 & 1.6 & 1.5 & 1.0 \\
\cline { 2 - 5 } & 2.0 & 0.6 & 1.5 & 1.0 \\
\cline { 2 - 5 } & 2.8 & 1.4 & 1.3 & 1.6 \\
\hline \multirow{3}{*}{ MMOI 3 } & 0.5 & 1.2 & 1.6 & 0.7 \\
\cline { 2 - 5 } & 1.1 & 1.5 & 1.4 & 0.5 \\
\cline { 2 - 5 } & 0.7 & 1.3 & 1.7 & 0.5 \\
\hline
\end{tabular}

Table 5-19: ANOVA calculations of MMOI and size effects on angular velocity.

\begin{tabular}{|ccccccc|}
\hline $\begin{array}{c}\text { Source of } \\
\text { Variation }\end{array}$ & $S S$ & $d f$ & $M S$ & $F$ & P-value & $F$ crit \\
\hline Sample & 3.1 & 2 & 1.5 & 17.5 & $2.1 \mathrm{E}-05$ & 3.4 \\
Columns & 4.4 & 3 & 1.5 & 16.8 & $4.4 \mathrm{E}-06$ & 3.0 \\
Interaction & 3.4 & 6 & 0.6 & 6.4 & $3.9 \mathrm{E}-04$ & 2.5 \\
Within & 2.1 & 24 & 0.1 & & & \\
Total & 13.0 & 35 & & & & \\
\hline
\end{tabular}

Similar to translational velocity ANOVA values, angular velocity is greatly affected by size of the block relative to MMOI. Although MMOI and interaction have effects on the system, Columns again shows the highest influence.

The ANOVA analysis relies on values rather than coordinates for calculations. Therefore, the coordinates from the grid were assigned values based on their accuracy. Figure 5.45 shows how coordinate values were assigned based on location to the center. The closest values to the center, orange region, were assigned a value of 1 . Blocks that impacted in the next closest section, gray, were assigned a value of 2. Finally, the furthest region, blue, received a value of 3 . These values can be arbitrary numbers, as long as they remain consistent for the sections, because the 
ANOVA analysis was only calculating for interaction between the factors, rather than weighted quantities.

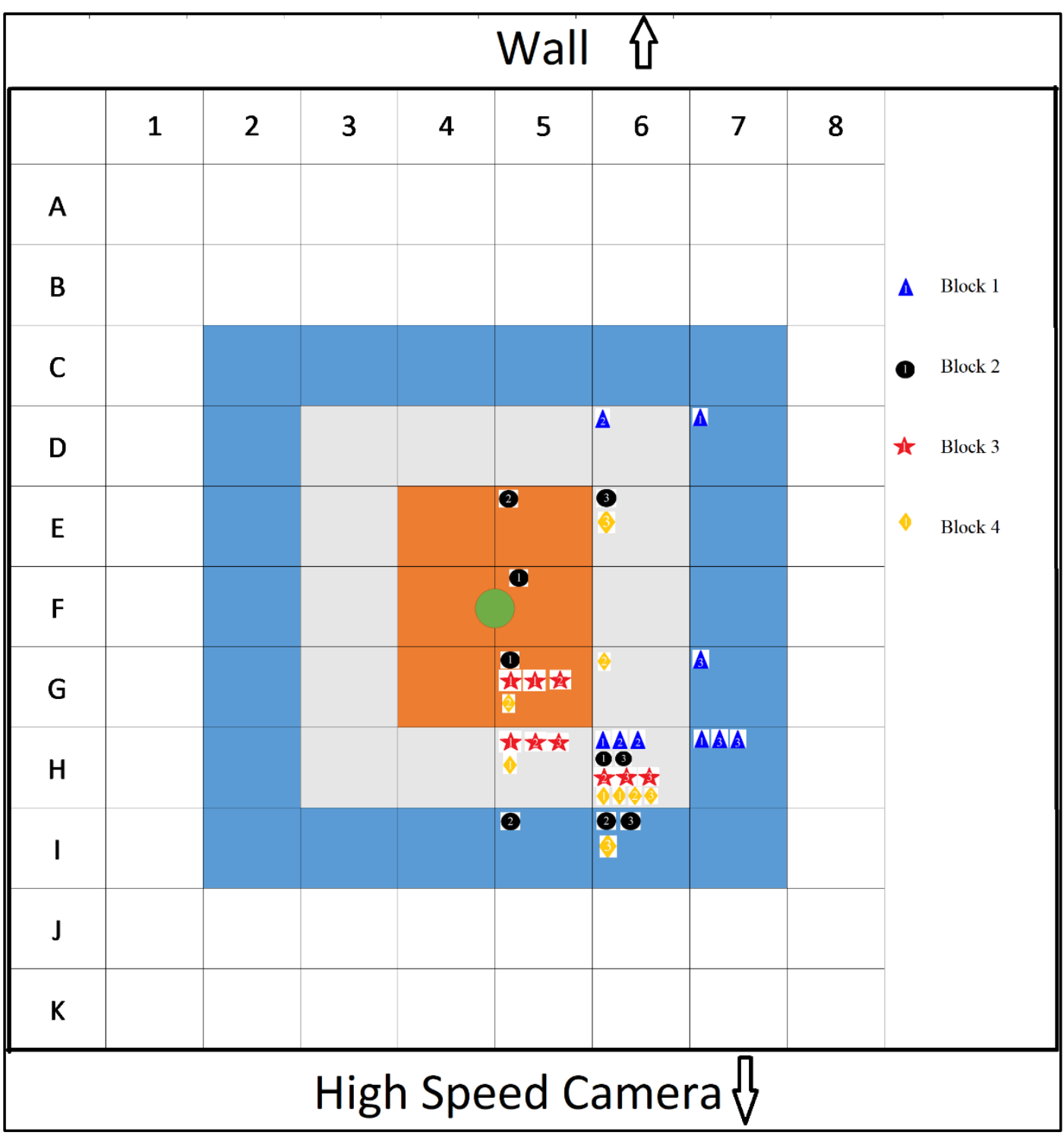

Figure 5.45: Assigned coordinate values for drop coordinates for ANOVA analysis. Where the Orange $=1$, Gray $=$ 2, Blue $=3$ 
Table 5-20: Drop test block landing locations collected for ANOVA analysis.

\begin{tabular}{|c|c|c|c|c|}
\hline \multirow{4}{*}{} & \multicolumn{5}{|c|}{ landing location } \\
\cline { 2 - 5 } & \multicolumn{4}{|c|}{ Size } \\
\cline { 2 - 5 } & Block 1 & Block 2 & Block 3 & Block 4 \\
\hline \multirow{3}{*}{ MMOI 1 } & 3 & 1 & 1 & 2 \\
\cline { 2 - 5 } & 3 & 1 & 1 & 2 \\
\cline { 2 - 5 } & 2 & 2 & 2 & 2 \\
\hline \multirow{3}{*}{ MMOI 2 } & 2 & 1 & 1 & 1 \\
\cline { 2 - 5 } & 2 & 3 & 2 & 2 \\
\hline \multirow{3}{*}{ MMOI 3 } & 3 & 2 & 2 & 2 \\
\cline { 2 - 5 } & 3 & 2 & 2 & 3 \\
\hline
\end{tabular}

Table 5-21: ANOVA calculations of MMOI and size effects on landing location.

\begin{tabular}{|ccccccc|}
\hline $\begin{array}{c}\text { Source of } \\
\text { Variation }\end{array}$ & $S S$ & $d f$ & $M S$ & $F$ & P-value & F crit \\
\hline Sample & 2.4 & 2 & 1.2 & 3.9 & $3.4 \mathrm{E}-02$ & 3.4 \\
Columns & 3.7 & 3 & 1.2 & 4.0 & $1.9 \mathrm{E}-02$ & 3.0 \\
& & & & & & \\
Interaction & 2.5 & 6 & 0.4 & 1.4 & $2.7 \mathrm{E}-01$ & 2.5 \\
Within & 7.3 & 24 & 0.3 & & & \\
Total & 15.9 & 35 & & & & \\
\hline
\end{tabular}

Data from the impact location analysis shows that both size and MMOI have some effect on where the block will land. This translates to the size and MMOI also affected how soon the block tended to tumble. However, at faster initial rotational rates, the body remained more spin stale, causing less of an effect on landing location.

To better understand the vertical drop test data, other factors that may have influenced the data were also analyzed. First, Froude number was calculated to determine its relationship to other dimensionless angular velocity. Froude number was calculated using the relationship:

$$
F r=\frac{u^{2}}{L g}
$$

Where $u$ is the average translational velocity calculated from Equation 5-18, $L$ is the length scale of $S A / L$, and $g$ is the acceleration due to gravity. From Figure 5.46, it can be seen that the Froude 
number directly relates to the dimensionless angular velocity; thus, Froude number is related to the velocity and geometry of the block. Therefore, it was important to analyze the virtual mass of each block to determine its effects.

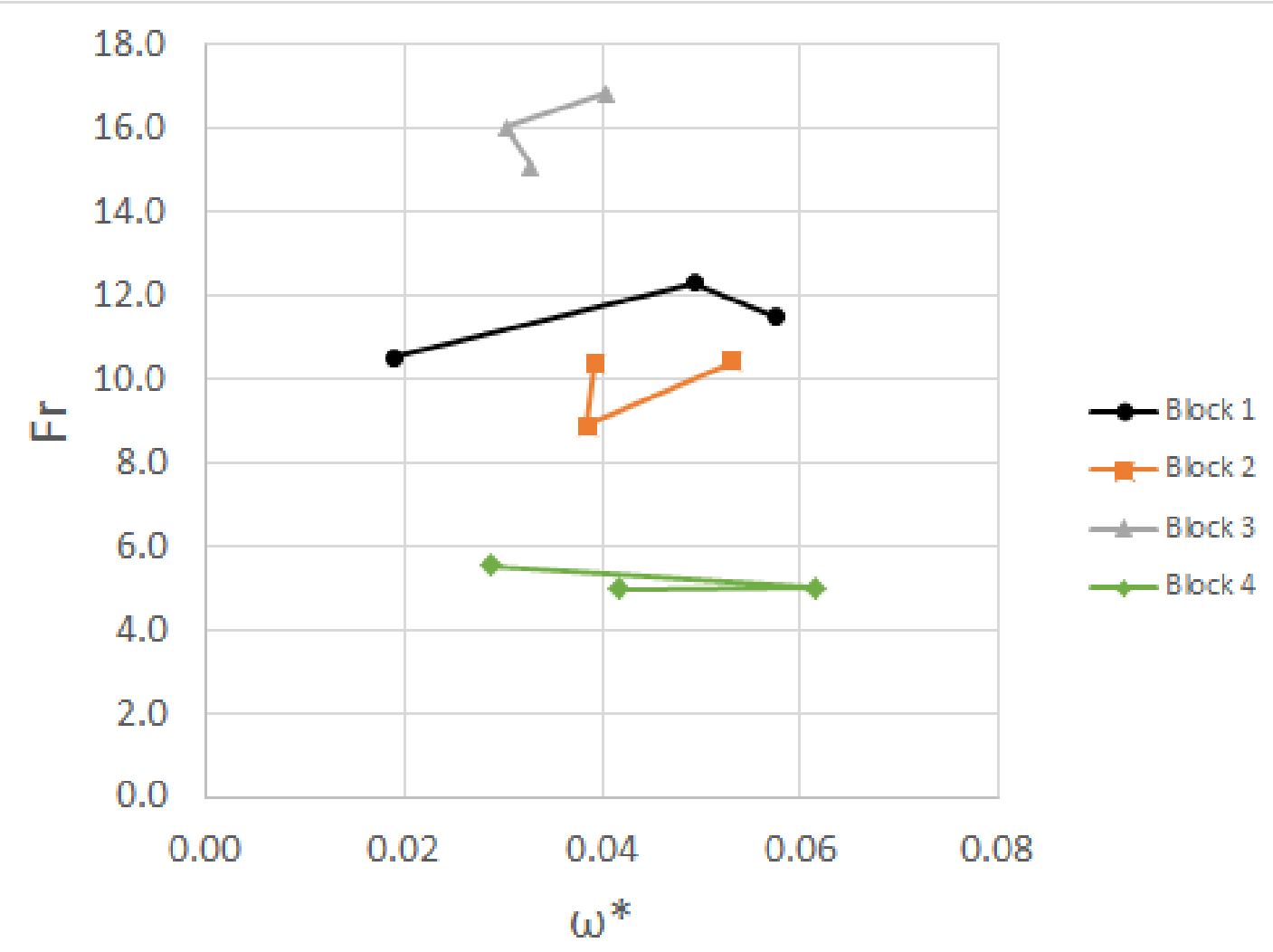

Figure 5.46: Froude number calculation for drop tests.

The virtual mass is a measurement of the force on a body due to its acceleration within a fluid [11]. The virtual mass was calculated for each block based on the relationship analyzed by Panye [12].

$$
I=\frac{\pi}{4} \rho_{f} l^{2} b
$$

Where $I$ is the virtual mass of the block, $\rho_{f}$ is the density of the fluid, $l$ is the thickness of the block, and $b$ is the largest dimension of the block. Table 5-22 shows the calculations of virtual mass for each of the blocks used. The largest percent of total mass any virtual mass obtained was that of block 4, with a virtual mass of 0.93 grams. Since the virtual mass of each block was small relative to the block's total mass, it was deemed insignificant for calculation purposes. 
Table 5-22: Virtual mass calculation for block sizes.

\begin{tabular}{|c|c|c|c|}
\hline Block Size & $\begin{array}{c}\text { Total Mass } \\
{[\mathrm{g}]}\end{array}$ & $\begin{array}{c}\text { Virtual } \\
\text { Mass } \\
{[\mathrm{g}]}\end{array}$ & $\begin{array}{c}\text { Percent of } \\
\text { Total Mass }\end{array}$ \\
\hline Block 1 & 95 & 0.35 & 0.37 \\
\hline Block 2 & 473 & 1.24 & 0.26 \\
\hline Block 3 & 848 & 1.49 & 0.18 \\
\hline Block 4 & 164 & 0.93 & 0.57 \\
\hline
\end{tabular}

\subsection{Throwing Mechanism with Photron SA5}

Testing with the throwing mechanism involved launching the four different block sizes and varying the MMOI between runs. Three randomized runs were conducted, and the values of each run were averaged to increase accuracy. The average translational velocity and angular velocity for each run were determined using the sum of all values divided by the total amount of data points. Data points were calculated by evaluating high speed video in the software Tracker. The highspeed video was recorded at $500 \mathrm{~Hz}$ at a shutter speed of $1000 \mathrm{~Hz}$. To acquire consistent data, a coordinate system and length scale were defined. The default coordinate system was placed at the hinge point of the throwing mechanism. The hinge point is approximately in plane with the point at which the block detaches from the throwing mechanism. This references frame was used to calculate the x-component of the translational velocity. The length scale was defined using a consistent, known measurement. It was ideal to use a length scale within the same plane as the block as it rested in the launcher. However, to get an accurate calibration length, the high contrast cabinmate door was used to calibrate the length. A test was done with a known length scale within the plane of the block to determine the accuracy of this calibration length. Using the longest side of the block as the calibration length, the translational velocities were measured to have a percent difference of approximately $2.3 \%$. The angular velocity was not affected because it was calculated relative to mass A. The cabinet door behind the launcher was measured to be $23.25 \mathrm{~cm}$. The user defined coordinate system and length scale are shown in Figure 5.47. 


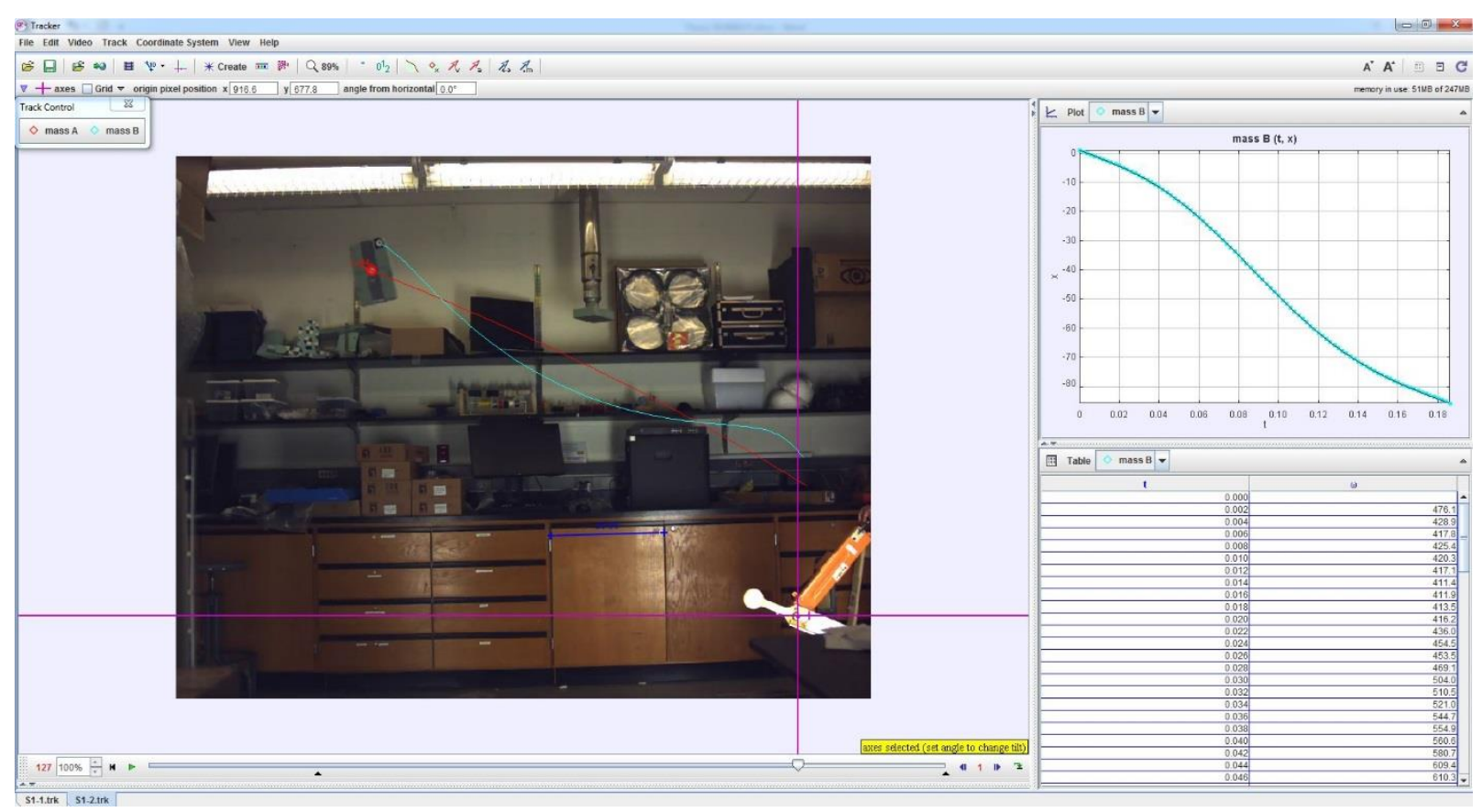

Figure 5.47: User defined reference coordinate system, placed at the hinge point of the throwing mechanism. Additionally, the projected paths of the points of interest and user defined scale.

Similar to the other data analyses conducted using Tracker, the user defined length scale introduced error in the data. To keep the length scale consistent and accurate, it was necessary to first zoom in on the image as far as possible. Then, one side of the adjustable scale was place on the edge of the cabinet. Finally, the other end was stretched to the opposite edge of the cabinet. Data collected from the high-speed video was used to calculate the dimensionless terms based on the length scales in Table 5-2.

Additionally, it was necessary to track the velocity of the throwing mechanism for testing. The velocity of the throwing mechanism for the SA5 analysis was later compared to its velocity during the Vicon analysis. Ideally, the average velocities of the thrower should have been similar. However, a scaling relationship that accounts for translational and rotational velocity should not depend on testing with similar velocity initial conditions. Between testing methods, the spring tension of the throwing mechanism was adjusted an unknown amount. Therefore, it was expected that the initial velocities would differ.

Using Tracker to track the tip of the thrower relative to its pivot point, an average angular velocity for the thrower was calculated over four runs. One run for each block size was analyzed and the average of the four tests was calculated for comparison to the throwing speed during Vicon analysis. Figure 5.48 demonstrates how the tip of the throwing arm was tracked relative to its 
pivot point. Multiple data points were collected near the point at which the block was released from the arm. The data points were averaged and collected in Table 5-23. From data in Table 5-23, the total average angular velocity of the throwing mechanism was calculated to be approximately $24 \mathrm{rad} / \mathrm{sec}$.

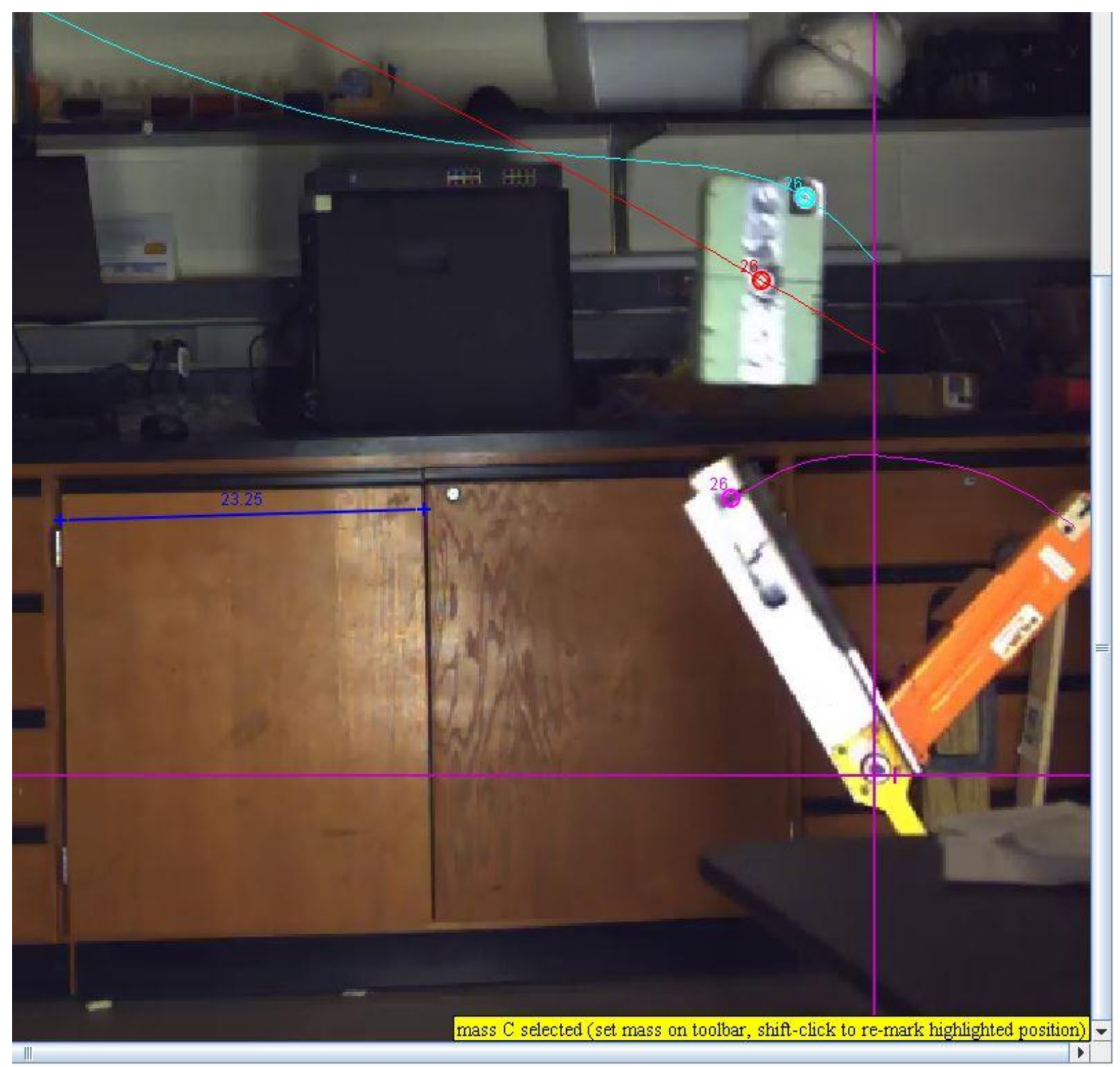

Figure 5.48: Tracking throwing ar angular velocity for SA5 tests to compare with Vicon tests. 
Table 5-23: Throwing arm average angular velocity data.

\begin{tabular}{|c|c|c|}
\hline \multicolumn{3}{|c|}{ Thrower Velocity } \\
\hline Trial & $\begin{array}{c}\text { Geometry of } \\
\text { block being } \\
\text { analyzed }\end{array}$ & $\begin{array}{c}\text { thrower angular } \\
\text { velocity } \\
{[\mathrm{rad} / \mathrm{sec}]}\end{array}$ \\
\hline Run 1 & Block 1 & 24 \\
\hline Run 2 & Block 2 & 22 \\
\hline Run 3 & Block 3 & 22 \\
\hline Run 4 & Block 4 & 27 \\
\hline
\end{tabular}

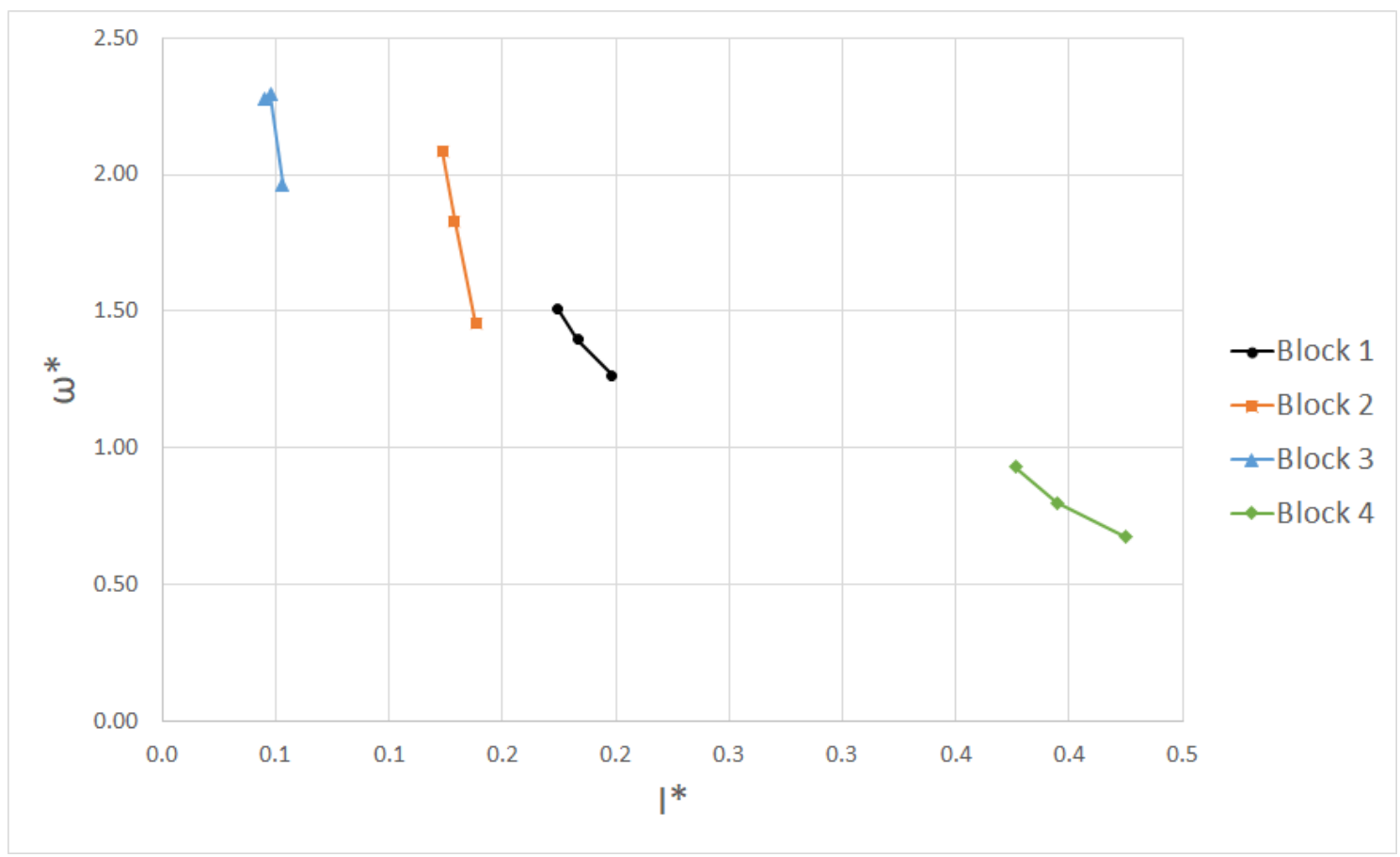

Figure 5.49: Dimensionless term comparison based on hydraulic diameter length scale. 


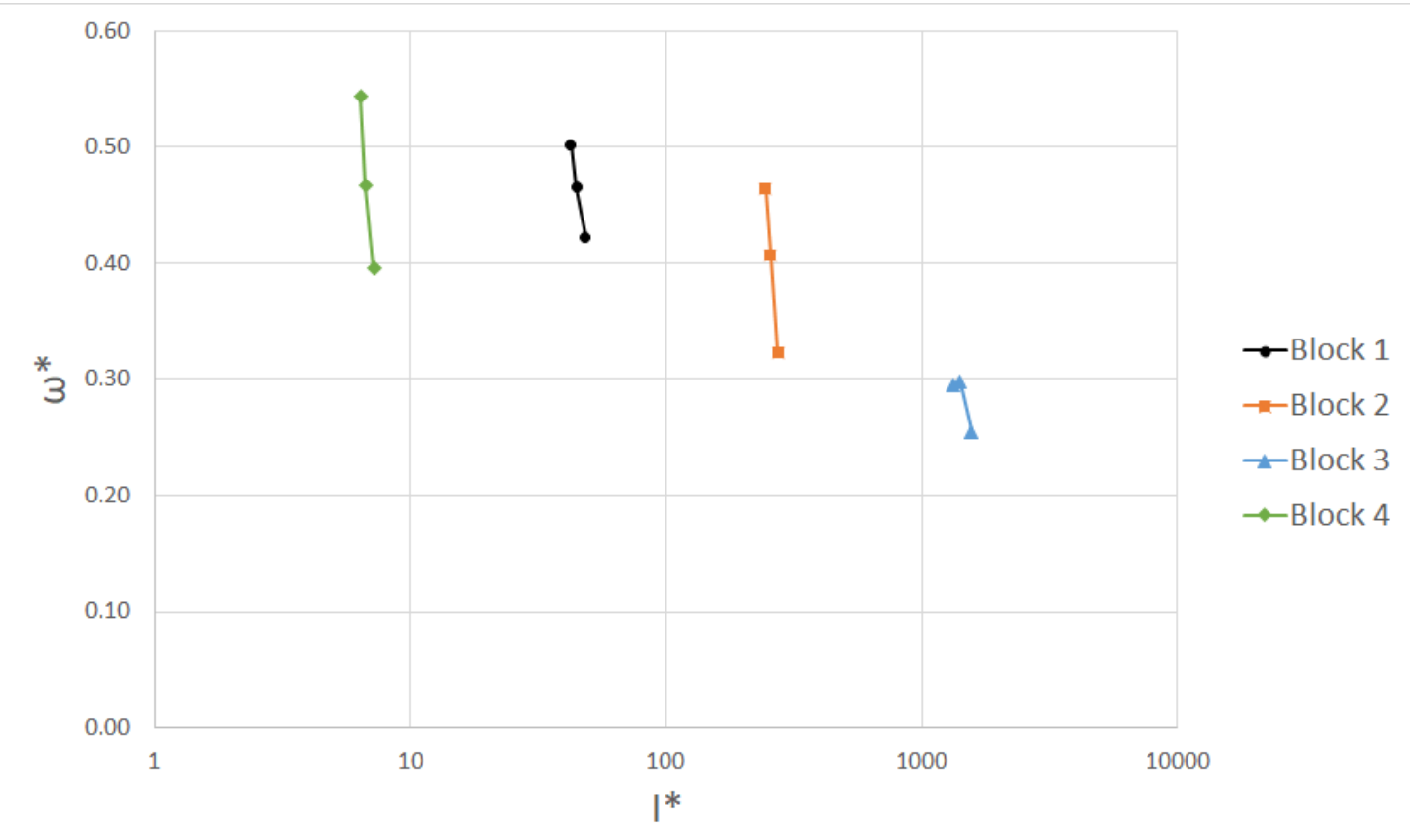

Figure 5.50: Dimensionless term comparison based on SA/L length scale.

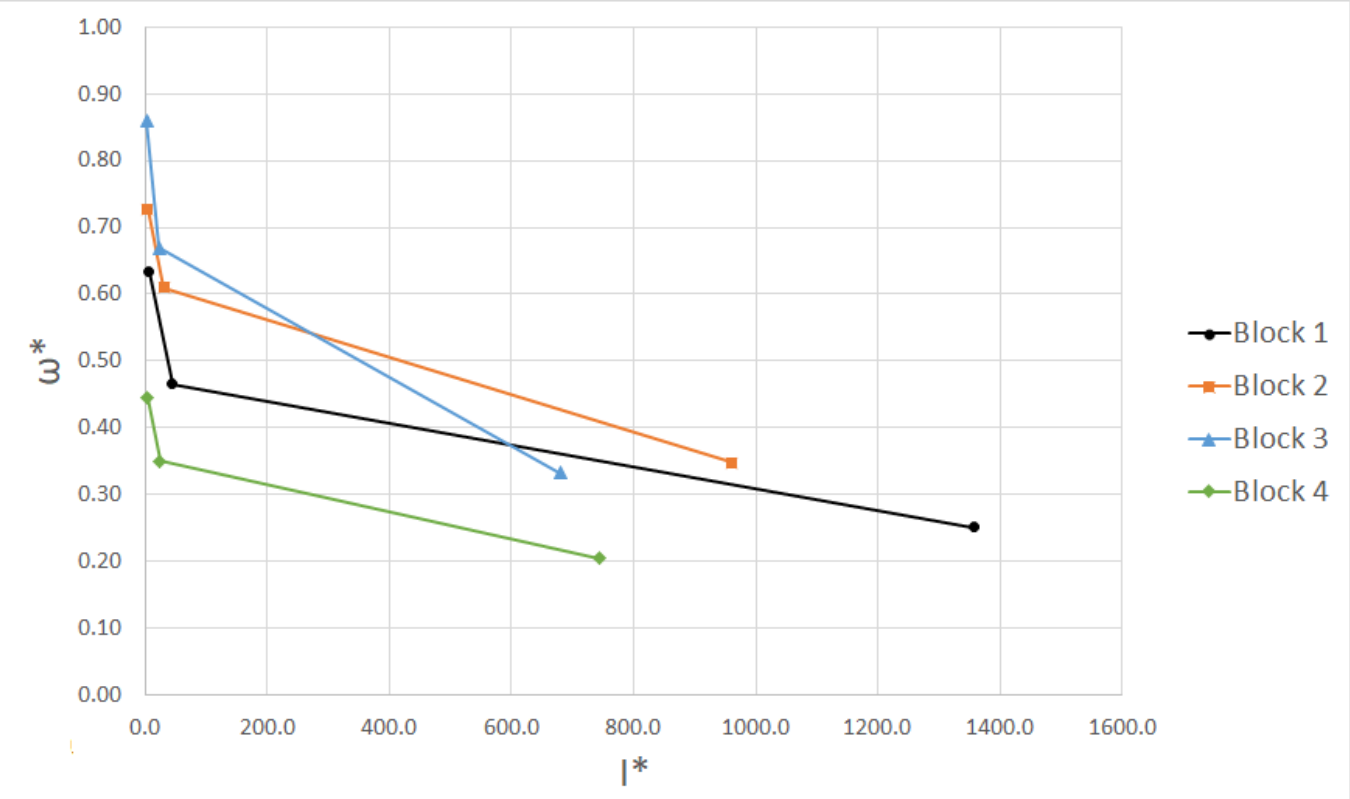

Figure 5.51: Dimensionless term comparison based on distant to bolt length scale. 


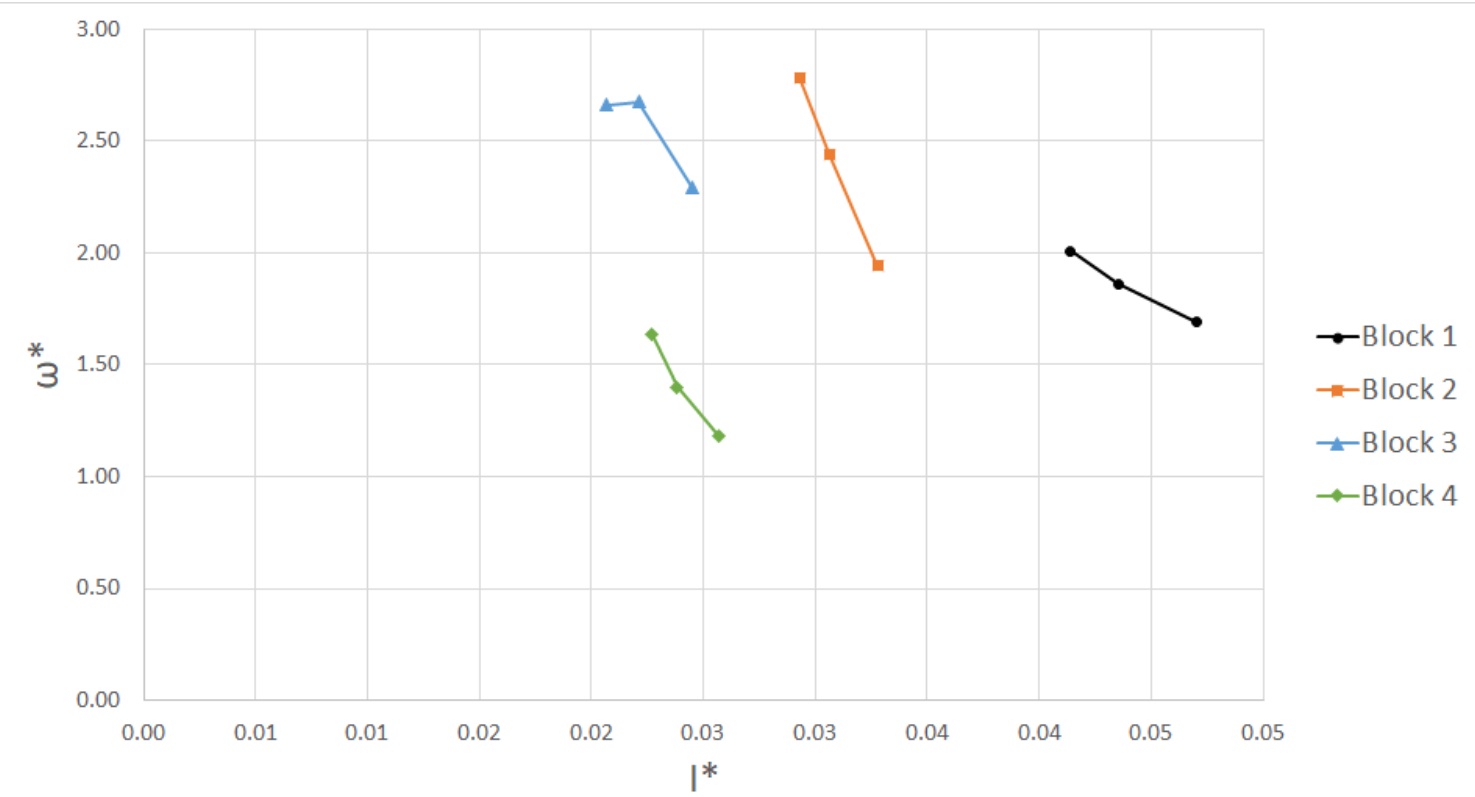

Figure 5.52: Dimensionless term comparison based on largest dimension, $W$, length scale.

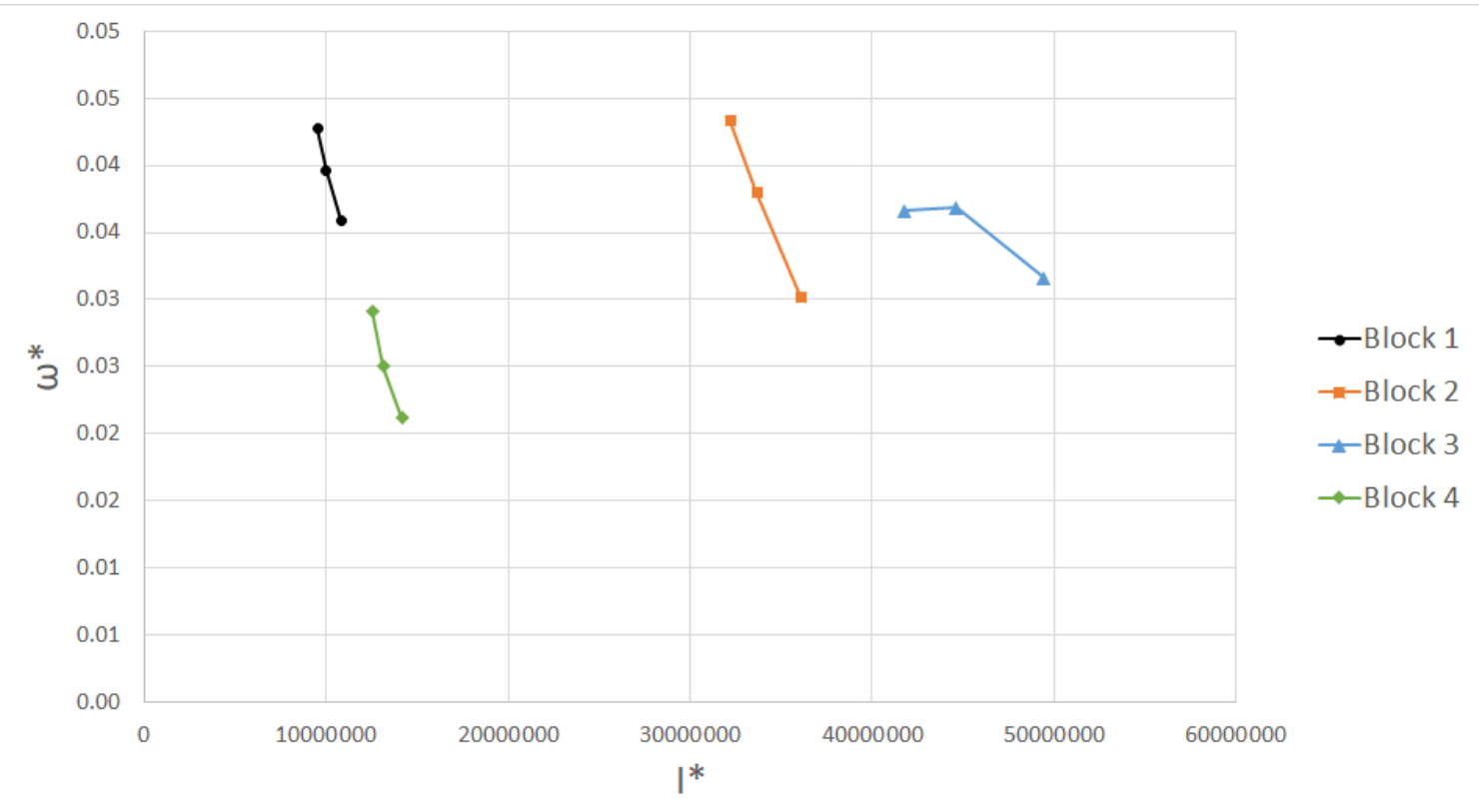

Figure 5.53: Dimensionless term comparison based on Volume/SA length scale. 


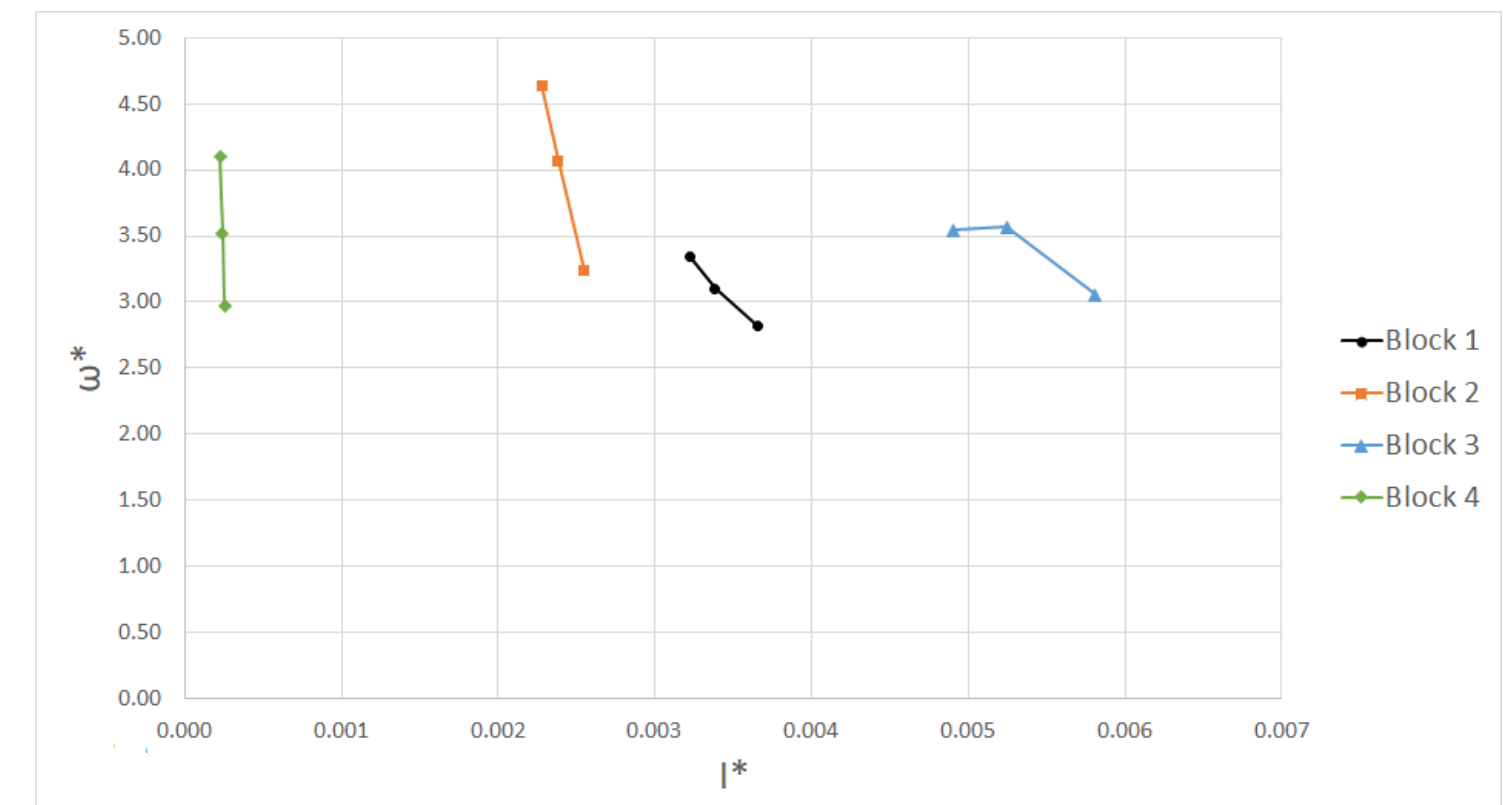

Figure 5.54: Dimensionless term comparison based on largest dimension, $W$, multiplied by aspect ratio length scale.

Based on data presented in Figure 5.49 through Figure 5.54, the length scales hydraulic diameter and $S A / L$ seem to follow a scaling relationship. To relate the dimensionless term data for each test method, the length scale $S A / L$ was used to analyze the data. An ANOVA analysis was performed to determine what factors affect the system. Table 5-24: Average translational velocity data collected from each run for ANOVA analysis. Table 5-24 and Table 5-25 display the average velocity values calculated for each run. This data was used for an ANOVA analysis, as in the previous testing methods. From the ANOVA data in Table 5-26, the factors that influenced translational velocity were easily determined. The source of variation Columns was the only factor to influence the data a significant amount. Recall that the data set Columns refers to the block geometry. Therefore, the ANOVA analysis showed that, for the case of MMOI and interaction, the null hypothesis could be rejected. Block geometry did, however, greatly affect the data. This could be due to the geometry of the blocks or the relative change in MMOI due to block size. An ANOVA analysis was also performed on rotational velocity of the blocks. Table 5-27 shows that both MMOI, geometry, and interaction affected the data. These affects were expected due to the large influence MMOI had on rotation of the blocks. 
Table 5-24: Average translational velocity data collected from each run for ANOVA analysis.

\begin{tabular}{|c|c|c|c|c|}
\hline \multirow{4}{*}{} & \multicolumn{4}{|c|}{$\mathbf{U}_{\mathbf{x}}$ [cm/sec] } \\
\cline { 2 - 5 } & \multicolumn{4}{|c|}{ Size } \\
\cline { 2 - 5 } & Block 1 & Block 2 & Block 3 & Block 4 \\
\hline \multirow{3}{*}{ MMOI 1 } & 484 & 378 & 334 & 544 \\
\cline { 2 - 5 } & 480 & 382 & 353 & 596 \\
\cline { 2 - 5 } & 420 & 370 & 357 & 627 \\
\hline \multirow{3}{*}{ MMOI 2 } & 497 & 370 & 347 & 566 \\
\cline { 2 - 5 } & 471 & 396 & 349 & 622 \\
\cline { 2 - 5 } & 490 & 395 & 328 & 601 \\
\hline \multirow{3}{*}{ MMOI 3 } & 509 & 418 & 359 & 579 \\
\cline { 2 - 5 } & 479 & 418 & 357 & 576 \\
\cline { 2 - 5 } & 477 & 406 & 376 & 584 \\
\hline
\end{tabular}

Table 5-25: Average angular velocity data collected from each run for ANOVA analysis.

\begin{tabular}{|l|c|c|c|c|}
\hline \multirow{4}{*}{} & \multicolumn{4}{|c|}{ [ [rad/sec] } \\
\cline { 2 - 5 } & \multicolumn{4}{|c|}{ Size } \\
\cline { 2 - 5 } & Block 1 & Block 2 & Block 3 & Block 4 \\
\hline \multirow{3}{*}{ MMOI 1 } & 36.0 & 21.1 & 14.0 & 27.5 \\
\cline { 2 - 5 } & 36.0 & 19.2 & 15.1 & 24.4 \\
\cline { 2 - 5 } & 37.5 & 21.6 & 16.4 & 23.7 \\
\hline \multirow{3}{*}{ MMOI 2 } & 35.7 & 18.4 & 15.9 & 22.5 \\
\cline { 2 - 5 } & 35.4 & 18.0 & 13.1 & 21.4 \\
\cline { 2 - 5 } & 35.6 & 19.4 & 15.9 & 21.9 \\
\hline \multirow{3}{*}{ MMOI 3 } & 31.7 & 15.2 & 14.1 & 19.5 \\
\cline { 2 - 5 } & 32.7 & 15.2 & 14.1 & 16.4 \\
\cline { 2 - 5 } & 33.0 & 17.1 & 12.8 & 18.2 \\
\hline
\end{tabular}


Table 5-26: ANOVA data based on translational velocity data.

\begin{tabular}{|c|c|c|c|c|c|c|}
\hline ANOVA & & & & & & \\
\hline $\begin{array}{l}\text { Source of } \\
\text { Variation }\end{array}$ & SS & $d f$ & $M S$ & $F$ & P-value & F crit \\
\hline Sample & 1890.4 & 2 & 945.2 & 2.2 & $1.3 \mathrm{E}-01$ & 3.4 \\
\hline Columns & 297023.0 & 3 & 99007.7 & 234.8 & $6.5 \mathrm{E}-18$ & 3.0 \\
\hline Interaction & 2914.9 & 6 & 485.8 & 1.2 & $3.6 \mathrm{E}-01$ & 2.5 \\
\hline Within & 10120.0 & 24 & 421.7 & & & \\
\hline Total & 311948.3 & 35 & & & & \\
\hline
\end{tabular}

Table 5-27: ANOVA data based on rotational velocity data.

\begin{tabular}{|c|c|c|c|c|c|c|}
\hline ANOVA & & & & & & \\
\hline $\begin{array}{l}\text { Source of } \\
\text { Variation }\end{array}$ & SS & $d f$ & $M S$ & $F$ & P-value & F crit \\
\hline Sample & 118.4 & 2 & 59.2 & 44.8 & 7.9E-09 & 3.4 \\
\hline Columns & 2093.6 & 3 & 697.9 & 527.8 & 4.9E-22 & 3.0 \\
\hline Interaction & 25.7 & 6 & 4.3 & 3.2 & $1.8 \mathrm{E}-02$ & 2.5 \\
\hline Within & 31.7 & 24 & 1.3 & & & \\
\hline Total & 2269.5 & 35 & & & & \\
\hline
\end{tabular}

\subsection{Throwing Mechanism with VICON}

Testing using the Vicon camera system followed the same launching procedure as with the SA5. Three Runs at each MMOI were recorded at $500 \mathrm{~Hz}$. During the time between recording with the SA5 and Vicon system, the adjustable spring on the throwing mechanism was adjusted an unknown amount. Therefore, a comparison of average angular velocity of the thrower was also analyzed.

As previously mentioned, the Vicon software recorded body position in quaternions. The quaternion data was exported to a .csv file and converted to translational velocity and angular velocity using the MATLAB code attached in Appendix B [13]. This code imported the .csv file and plotted the translational velocities in each axis versus time as well as pitch rate, roll rate, and yaw rate. For the purposes of this study, only the pitch rate and translational velocity in the $x$-axis was desired. Additional axis data was used for a quantitative analysis of the block's deviation from the desired trajectory (i.e. how the velocities and angular velocities were distributed among the three axes). 
Data exported from Vicon was not trimmed. Therefore, the plots include time when the block was stationary in the launcher (Figure 5.55). To calculate average velocities, an average of the velocity data was taken ensuing the maximum velocity. Ideally, the velocity would reach a maximum velocity and remain constant. However, due to external forces, the block reached a maximum velocity as it was released from the launcher and proceeded to decrease velocity at a linear rate. Thus, the maximum velocity should occur just after the block was accelerated by the throwing mechanism. It was important to visually inspect the velocity plots to ensure the maximum velocity occurred immediately after the large increase in velocity. Noise in the data from missing time steps was able to cause the maximum velocity to appear later in the trajectory; as demonstrated in Figure 5.56. The maximum velocity in Figure 5.56 was at $t=0.25$, according to the tracker data. This was caused by the system missing a point during one or more of the time steps. The smoothed data, shown in red, corrected for the noise in the data and a more accurate maximum velocity was measured at approximately $t=0.02$, indicated by the “*”.

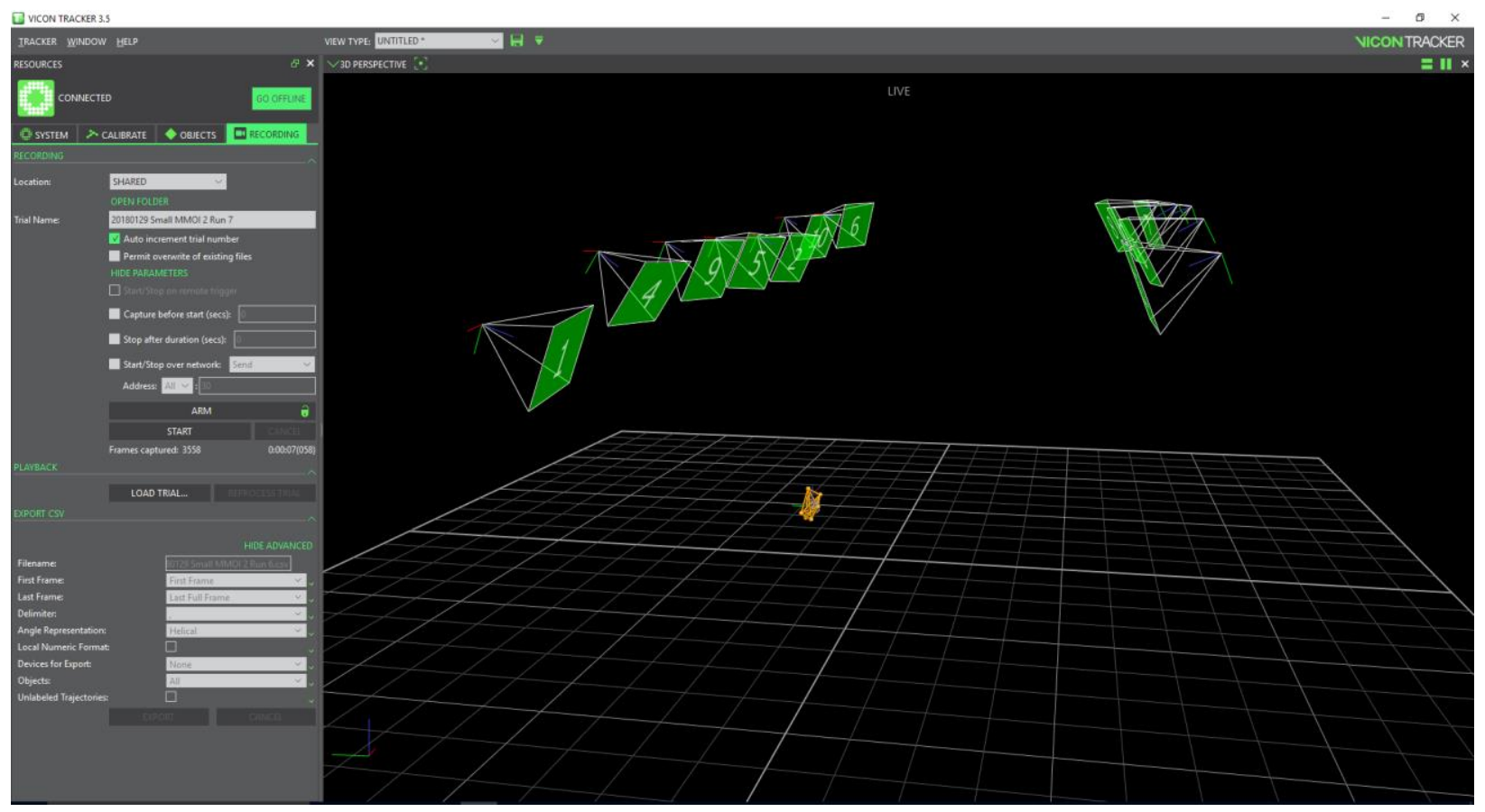

Figure 5.55: Object in launcher before motion was induced. 


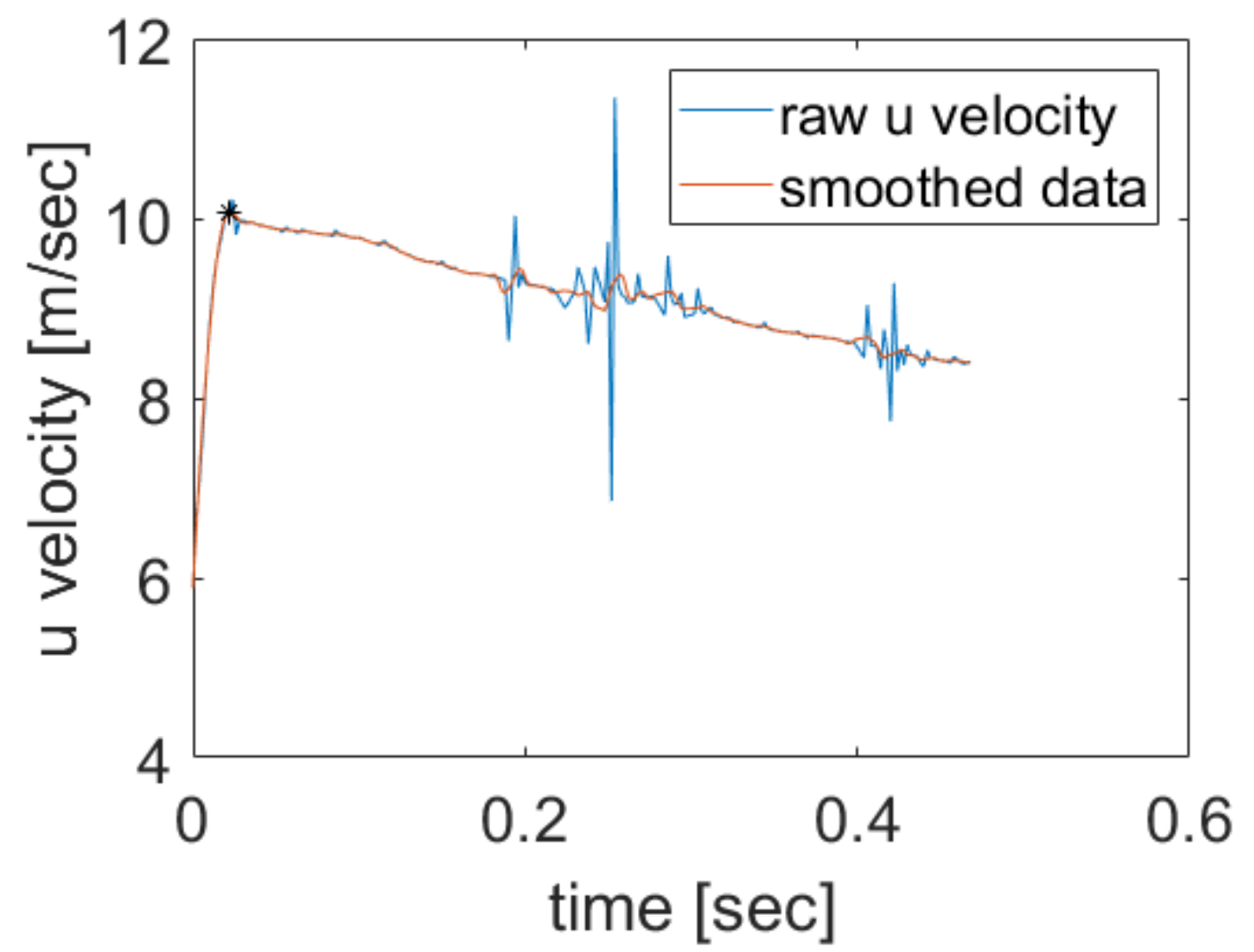

Figure 5.56: Translational velocity of the block vs. smoothed data to demonstrate noise in measurement.

To calculate velocities that better represented the motion of the blocks, the smoothing function was used on the data to reduce noise (Figure 5.56). From the smoothed data, the average velocities, shown in Table 5-28, were calculated. This smoothing technique was not necessary for the data collected from the previous experiments due to the relatively low noise in the data. Three values for the velocity data collected for Block 4 were considered inaccurate based on previous experiments. The data in Table 5-28 highlighted in red was not used to calculate the average angular velocity if the block. During these runs, the software was not able to accurately smooth the data, causing the excessive noise to greatly alter the data. Figure 5.57 demonstrates the excessive noise in the data for run 1 of MMOI 1 for Block 4. 
Table 5-28: Average velocity data calculated from smoothed data collected by Vicon system.

\begin{tabular}{|c|c|c|c|c|c|c|}
\hline \multicolumn{7}{|c|}{ Block 1} \\
\hline & \multicolumn{2}{|c|}{ MMOI 1} & \multicolumn{2}{|c|}{ MMOI 2} & \multicolumn{2}{|c|}{ MMOI 3} \\
\hline & $\mathrm{U}_{\mathrm{x}}[\mathrm{m} / \mathrm{sec}]$ & $\omega[\mathrm{deg} / \mathrm{sec}]$ & $\mathrm{U}_{\mathrm{x}}[\mathrm{m} / \mathrm{sec}]$ & $\omega[\mathrm{deg} / \mathrm{sec}]$ & $\mathrm{U}_{\mathrm{x}}[\mathrm{m} / \mathrm{sec}]$ & $\omega[\mathrm{deg} / \mathrm{sec}]$ \\
\hline Run 1 & 9.18 & 1854 & 8.05 & 1977 & 9.06 & 1778 \\
\hline Run 2 & 8.5 & 1761 & 8.12 & 1871 & 8.88 & 1544 \\
\hline Run 3 & 7.97 & 1963 & 8.67 & 2090 & 8.96 & 1809 \\
\hline Avg & 8.55 & 1859.33 & 8.28 & 1979.33 & 8.97 & 1710.33 \\
\hline \multicolumn{7}{|c|}{ Block 2} \\
\hline & \multicolumn{2}{|c|}{ MMOI 1} & \multicolumn{2}{|c|}{ MMOI 2} & \multicolumn{2}{|c|}{ MMOI 3} \\
\hline & $\mathrm{U}_{\mathrm{x}}[\mathrm{m} / \mathrm{sec}]$ & $\omega[\mathrm{deg} / \mathrm{sec}]$ & $\mathrm{U}_{\mathrm{x}}[\mathrm{m} / \mathrm{sec}]$ & $\omega[\mathrm{deg} / \mathrm{sec}]$ & $\mathrm{U}_{\mathrm{x}}[\mathrm{m} / \mathrm{sec}]$ & $\omega[\mathrm{deg} / \mathrm{sec}]$ \\
\hline Run 1 & 6.67 & 1012 & 7.08 & 882 & 6.68 & 650 \\
\hline Run 2 & 6.86 & 970 & 6.99 & 782 & 6.57 & 603 \\
\hline Run 3 & 6.63 & 1014 & 7.09 & 839 & 6.98 & 929 \\
\hline Avg & 6.72 & 998.67 & 7.05 & 834.33 & 6.74 & 727.33 \\
\hline \multicolumn{7}{|c|}{ Block 3} \\
\hline & \multicolumn{2}{|c|}{ MMOI 1} & \multicolumn{2}{|c|}{ MMOI 2} & \multicolumn{2}{|c|}{ MMOI 3} \\
\hline & $\mathrm{U}_{\mathrm{x}}[\mathrm{m} / \mathrm{sec}]$ & $\omega[\mathrm{deg} / \mathrm{sec}]$ & $\mathrm{U}_{\mathrm{x}}[\mathrm{m} / \mathrm{sec}]$ & $\omega[\mathrm{deg} / \mathrm{sec}]$ & $\mathrm{U}_{\mathrm{x}}[\mathrm{m} / \mathrm{sec}]$ & $\omega[\mathrm{deg} / \mathrm{sec}]$ \\
\hline Run 1 & 6.99 & 775 & 6.92 & 813 & 7.2 & 628 \\
\hline Run 2 & 6.81 & 873 & 6.99 & 789 & 7.31 & 655 \\
\hline Run 3 & 6.99 & 844 & 7.05 & 812 & 7.01 & 733 \\
\hline Avg & 6.93 & 830.67 & 6.99 & 804.67 & 7.17 & 672.00 \\
\hline \multicolumn{7}{|c|}{ Block 4} \\
\hline & \multicolumn{2}{|c|}{ MMOI 1} & \multicolumn{2}{|c|}{ MMOI 2} & \multicolumn{2}{|c|}{ MMOI 3} \\
\hline & $\mathrm{U}_{\mathrm{x}}[\mathrm{m} / \mathrm{sec}]$ & $\omega[\mathrm{deg} / \mathrm{sec}]$ & $\mathrm{U}_{\mathrm{x}}[\mathrm{m} / \mathrm{sec}]$ & $\omega[\mathrm{deg} / \mathrm{sec}]$ & $\mathrm{U}_{\mathrm{x}}[\mathrm{m} / \mathrm{sec}]$ & $\omega[\mathrm{deg} / \mathrm{sec}]$ \\
\hline
\end{tabular}




\begin{tabular}{|c|c|c|c|c|c|c|}
\cline { 2 - 7 } Run 1 & 6.09 & 786 & 6.07 & 761 & 5.46 & 1068 \\
\hline Run 2 & 5.52 & 653 & 6.23 & 1285 & 5.49 & 1127 \\
\hline Run 3 & 6.03 & 1259 & 5.83 & 1141 & 5.39 & 1275 \\
\hline Avg & 5.88 & 1259.00 & 6.04 & 1213.00 & 5.45 & 1156.67 \\
\hline
\end{tabular}

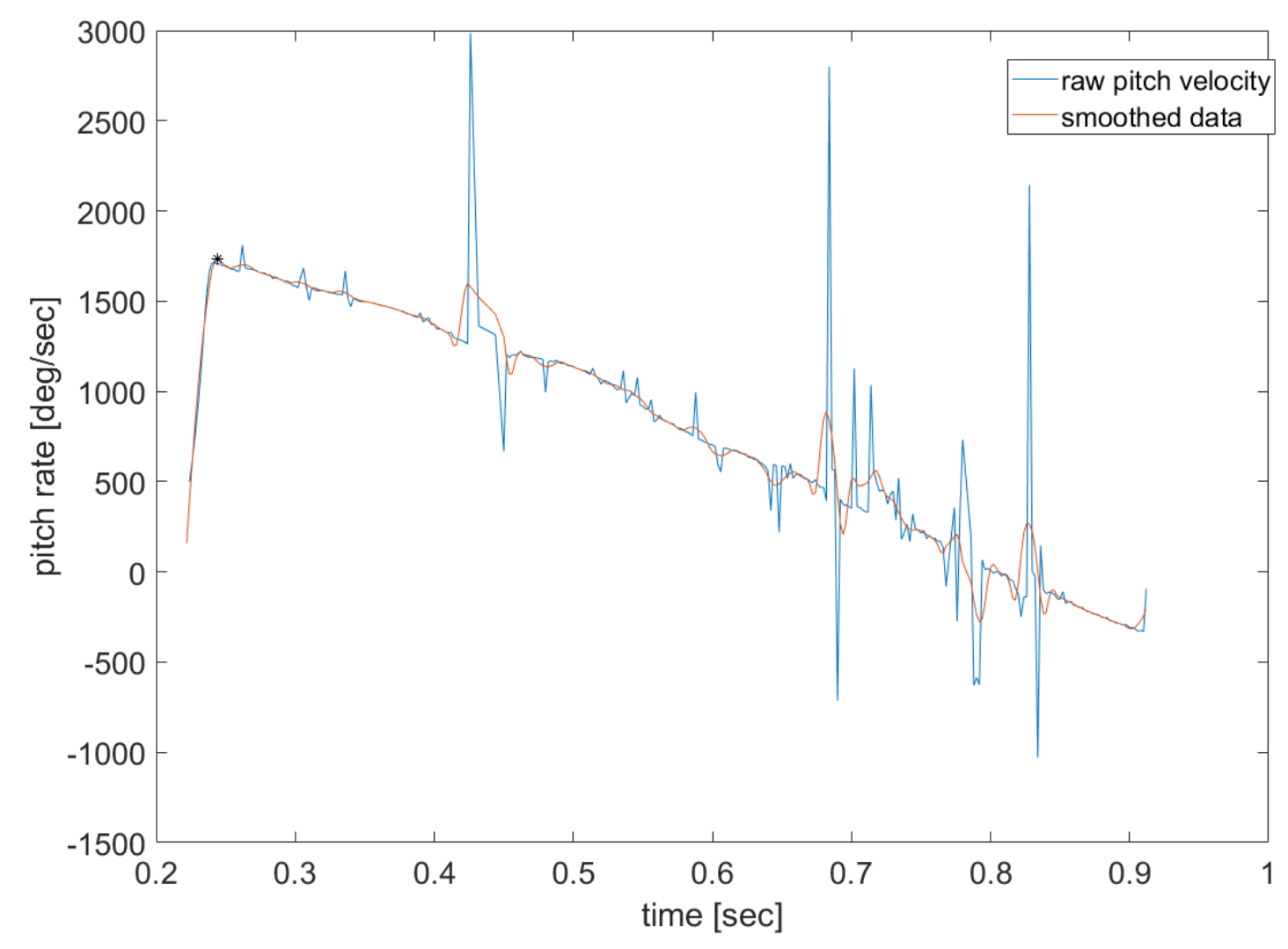

Figure 5.57: Excessive noise in run 1 of Block 4 for MMOI 1.

Other translational velocities and rotation rates were collected to quantify 3D motion. For each run, it was desirable to have minimal velocity in the $y$-axis and minimal rotation about the $z$ and $x$-axes, relative to the global coordinate system of the Vicon software. That is, only pitch rate of the block was analyzed, no yaw or roll rates were desired. It was expected to have translational velocity in the z-axis as the blocks were fired at an upward angle, then travelled downward in a parabolic trajectory. From Figure 5.58 and Figure 5.59 it is shown that the Downrange Velocity and Pitch Rate have the greatest magnitude. After smoothing, this data was averaged over the 
three runs for each MMOI and used in the dimensionless term scaling analysis. Smoothing was done on the data to reduce the amount of noise in the data. If the Vicon system lost the object for any number of frames, the software processed the object as moving a greater distance for the same timestep. The spikes in the data are caused by the software not being able to recreate the object due to masking, block orientation, etc.

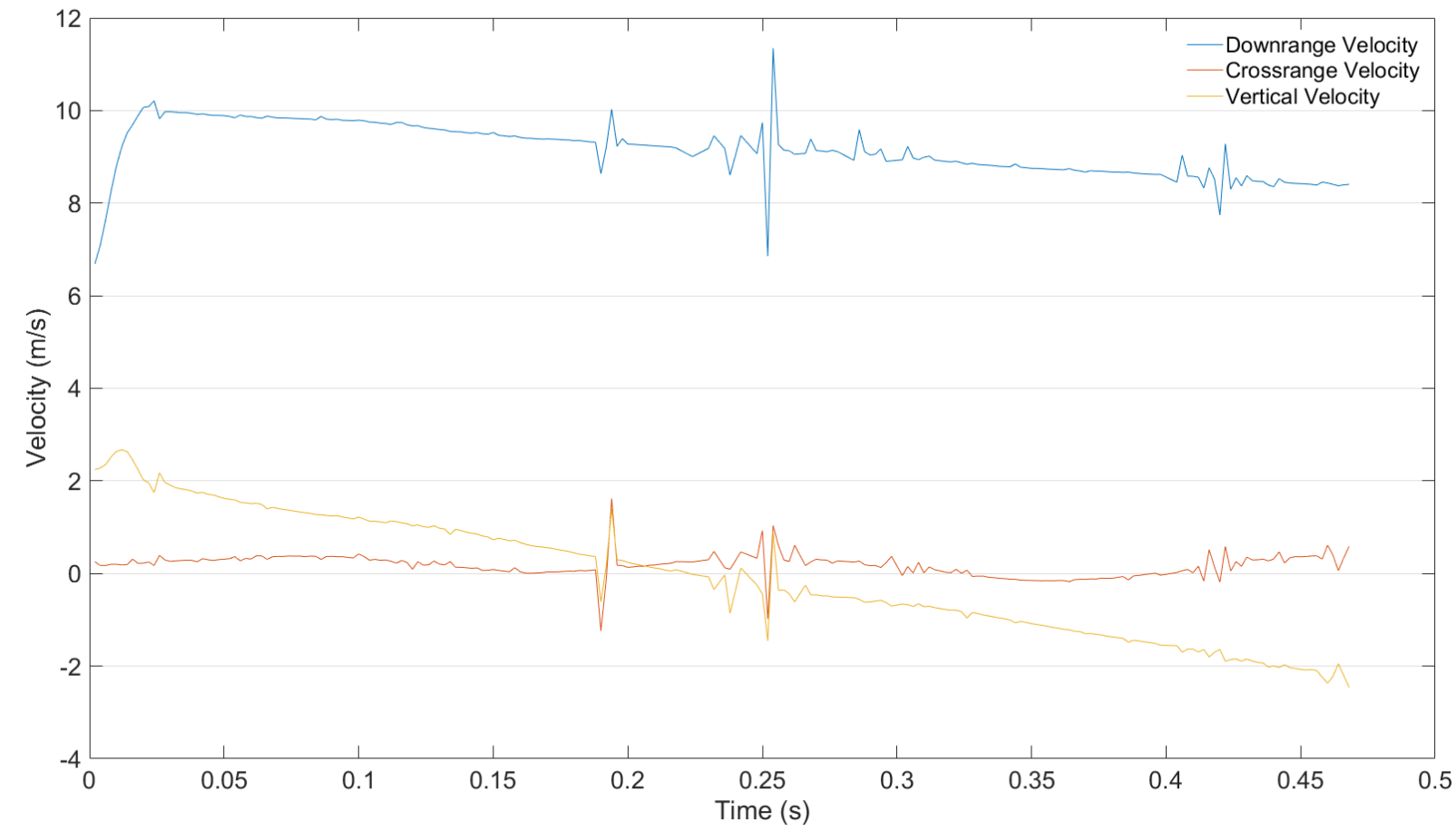

Figure 5.58: Raw translational data for Block 1- Run 1- MMOI 1 taken from Vicon system. Only Downrange Velocity in the $x$-axis was analyzed. 


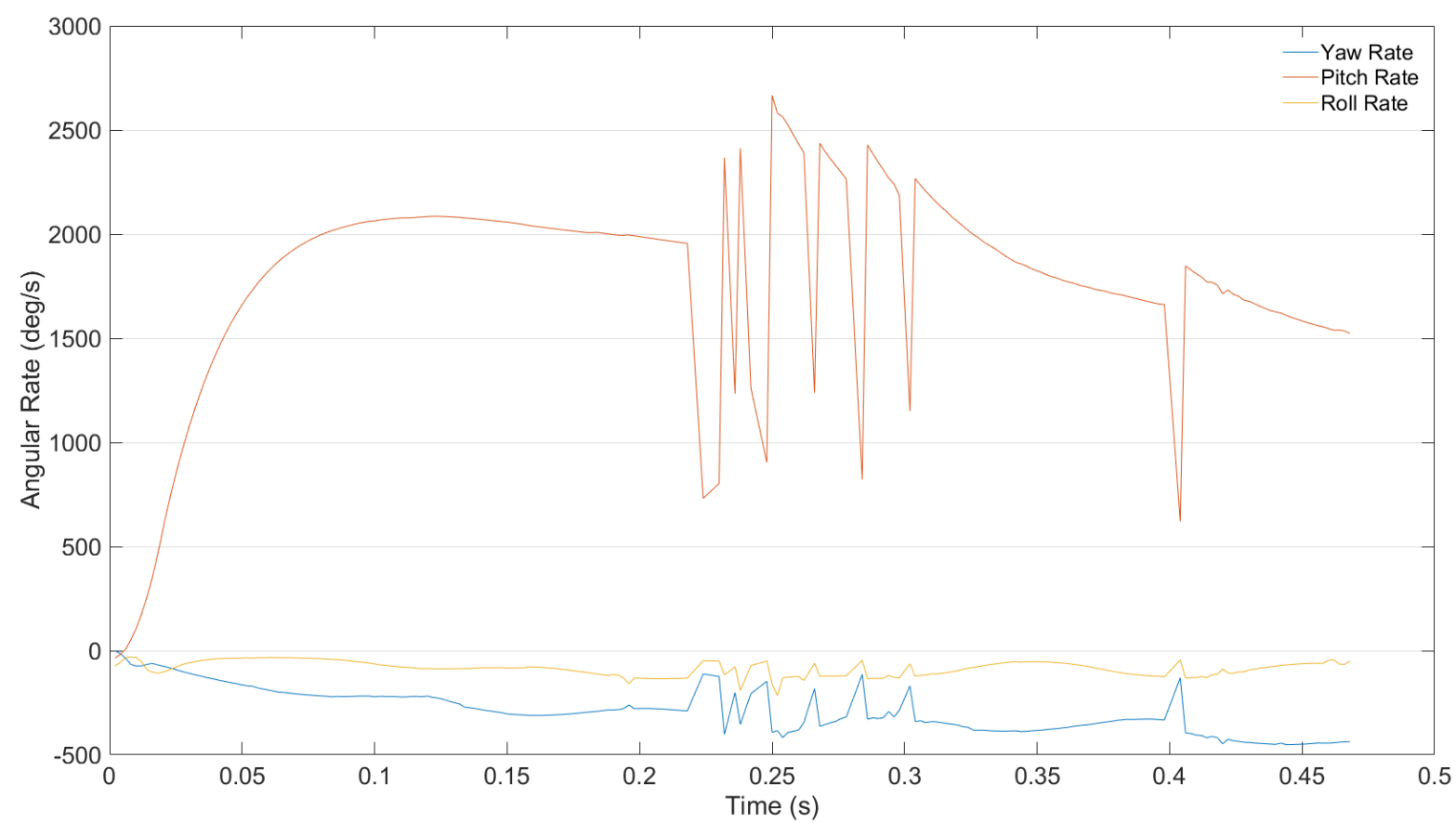

Figure 5.59: Raw rotational rate data for Block 1-Run 1- MMOI 1 taken from Vicon system. Only pitching rate about the y-axis was desired.

Similar to the analysis done using the SA5 high speed camera, the average angular velocity of the throwing arm was determined. Using the Vicon system to track reflective markers on the arm, data was collected for analysis of angular velocity relative to the pivot point of the launcher. Table 5-29 shows the data collected over three runs. The averages were calculated using 20 data points around the point at which the block was released from the launcher. The average velocity of the thrower was approximately $22 \mathrm{rad} / \mathrm{sec}$ and occurred at approximately $t=0.08$. However, the maximum velocity of the launcher occurred at approximately $t=0.15$ and averaged $57 \mathrm{rad} / \mathrm{sec}$. The maximum velocity did not affect the block due to it already being released from the arm. The maximum velocity occurring later in the rotation signifies that the launcher was accelerating during the release of the block.

Table 5-29: Tracker data for throwing arm angular velocity over three runs.

\begin{tabular}{|c|c|c|}
\hline & $\begin{array}{c}\text { Thrower angular velocity } \\
{[\mathrm{deg} / \mathrm{sec}]}\end{array}$ & $\begin{array}{c}\text { Thrower angular velocity } \\
{[\mathrm{rad} / \mathrm{sec}]}\end{array}$ \\
\hline Run 1 & 1247 & 22 \\
Run 2 & 1354 & 24 \\
Run 3 & 1225 & 21 \\
\hline Avg: & 1275 & 22 \\
\hline
\end{tabular}


Data from Table 5-28 was used to calculate dimensionless MMOI and dimensionless angular velocity. The data was compared using the length scales in Table 5-2.

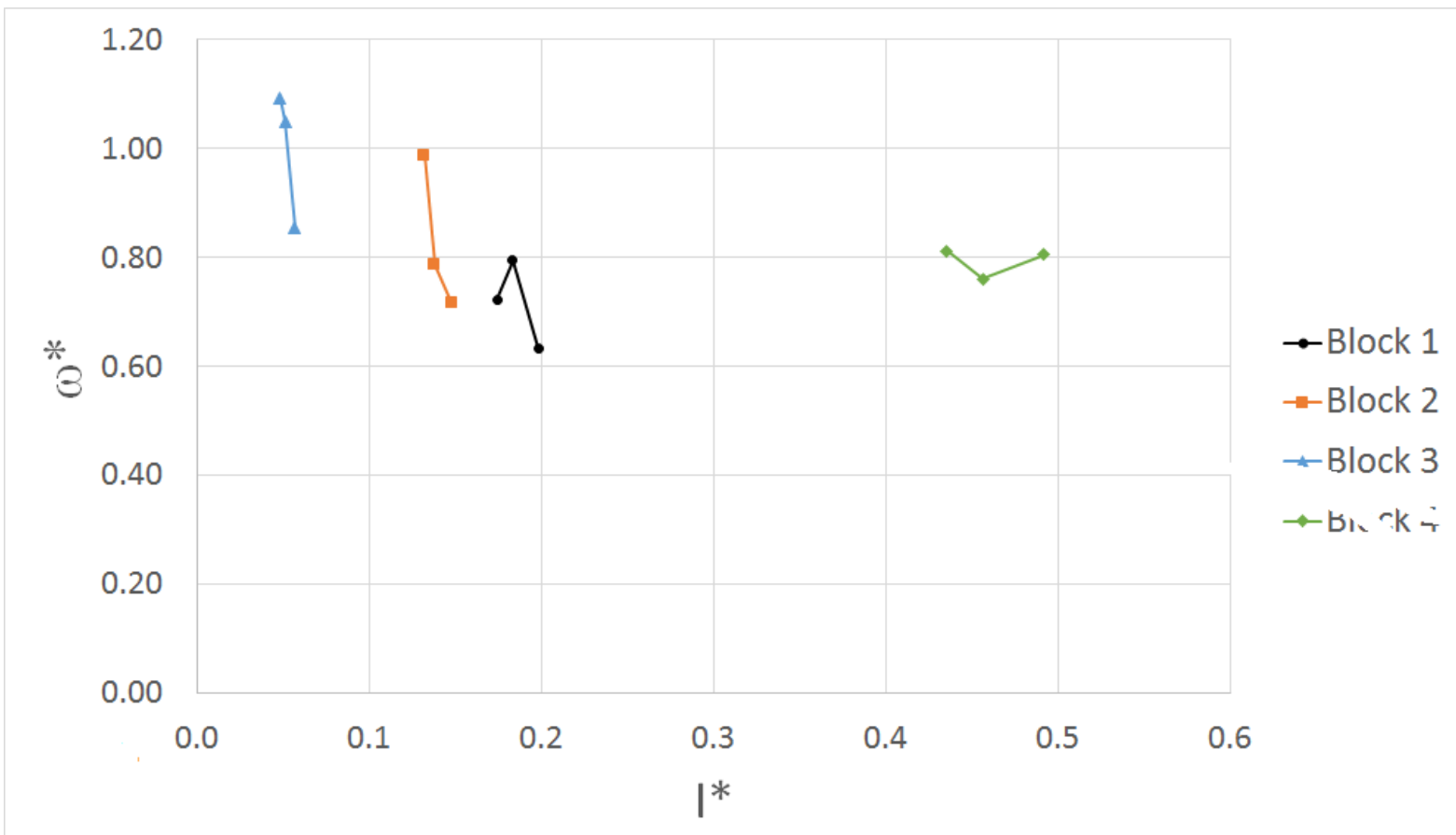

Figure 5.60: Hydraulic diameter length scale for Vicon high speed data.

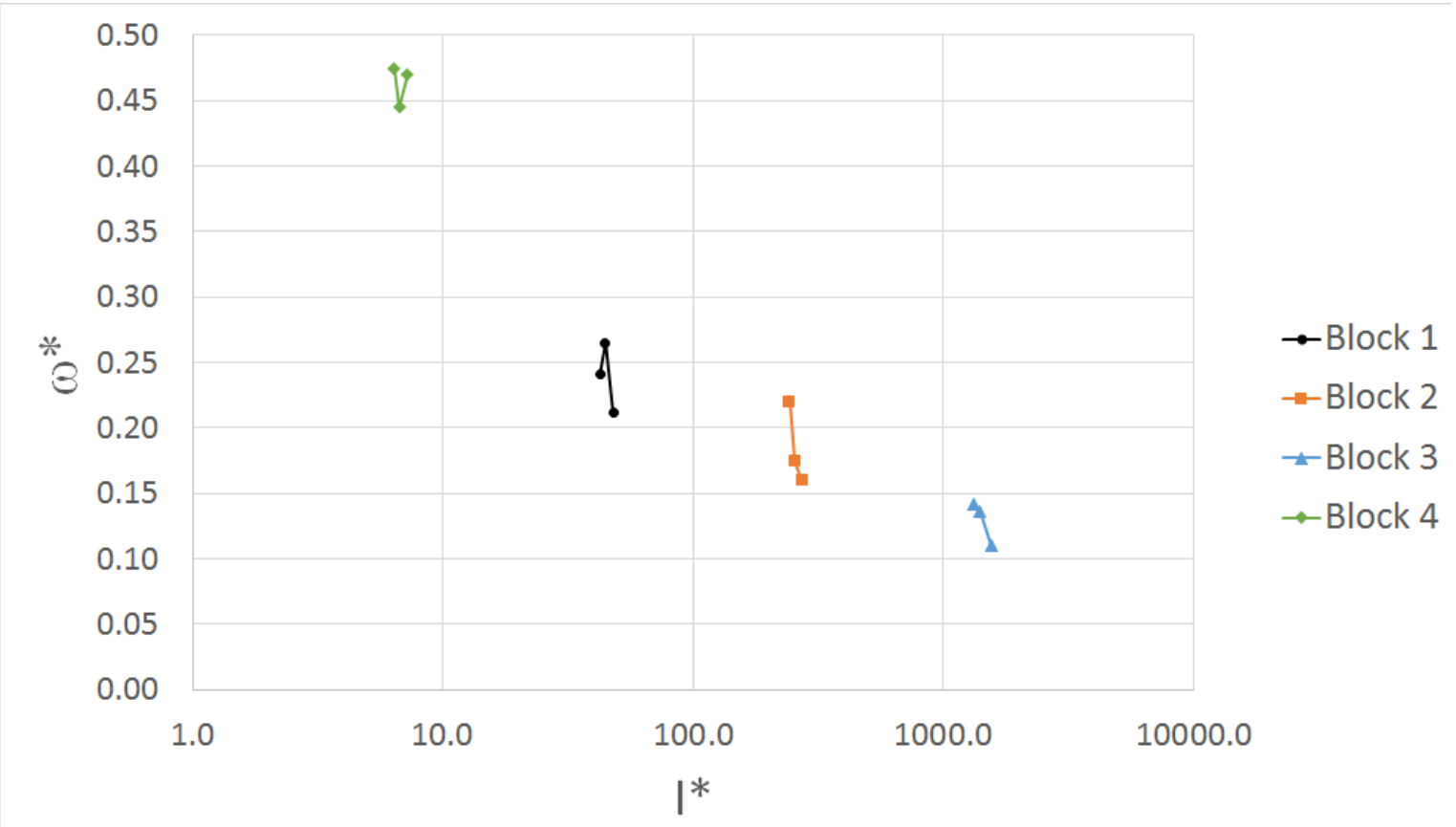

Figure 5.61: SA/L length scale for Vicon high speed data. 


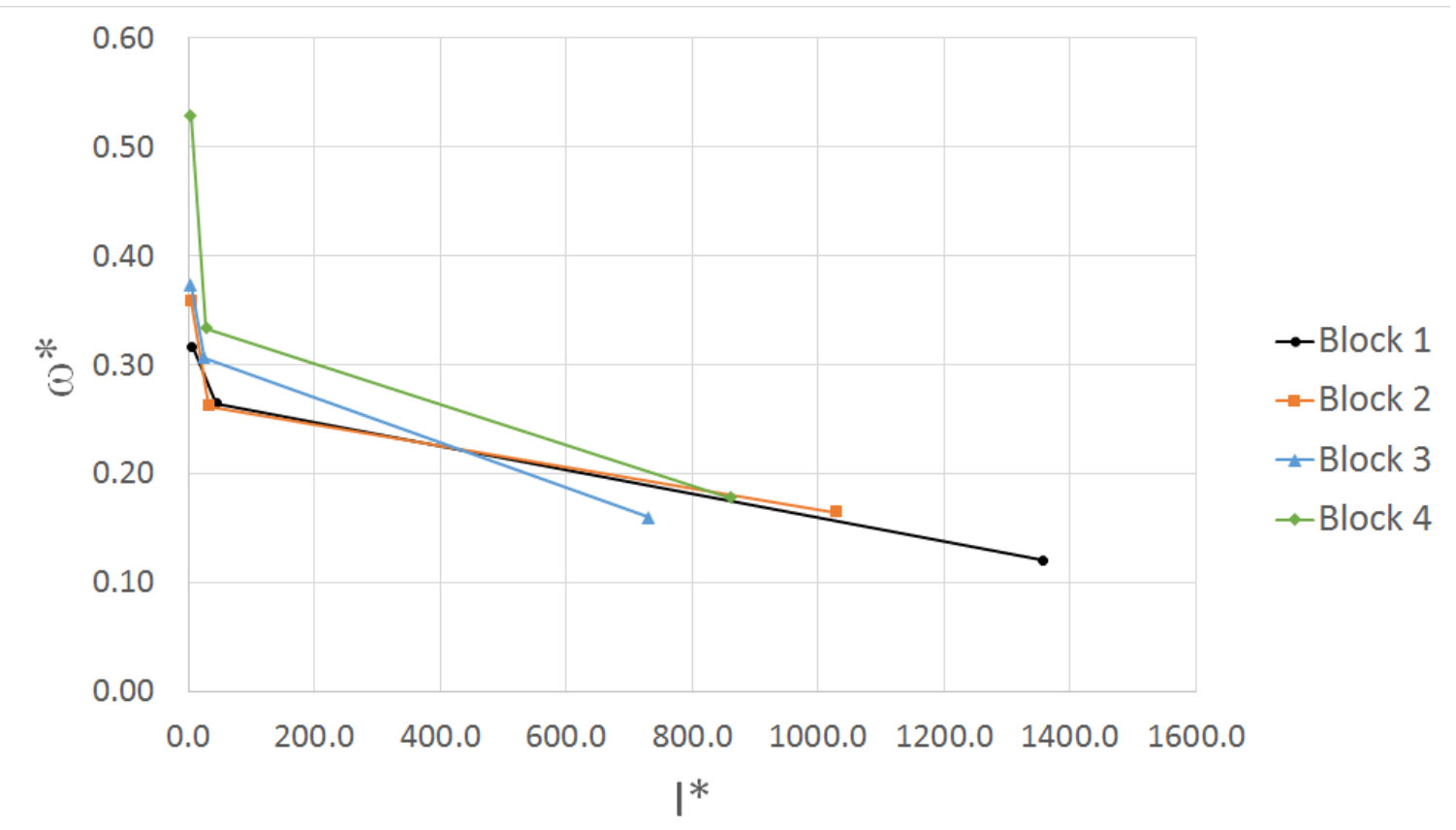

Figure 5.62: Distance to bolt length scale for Vicon high speed data.

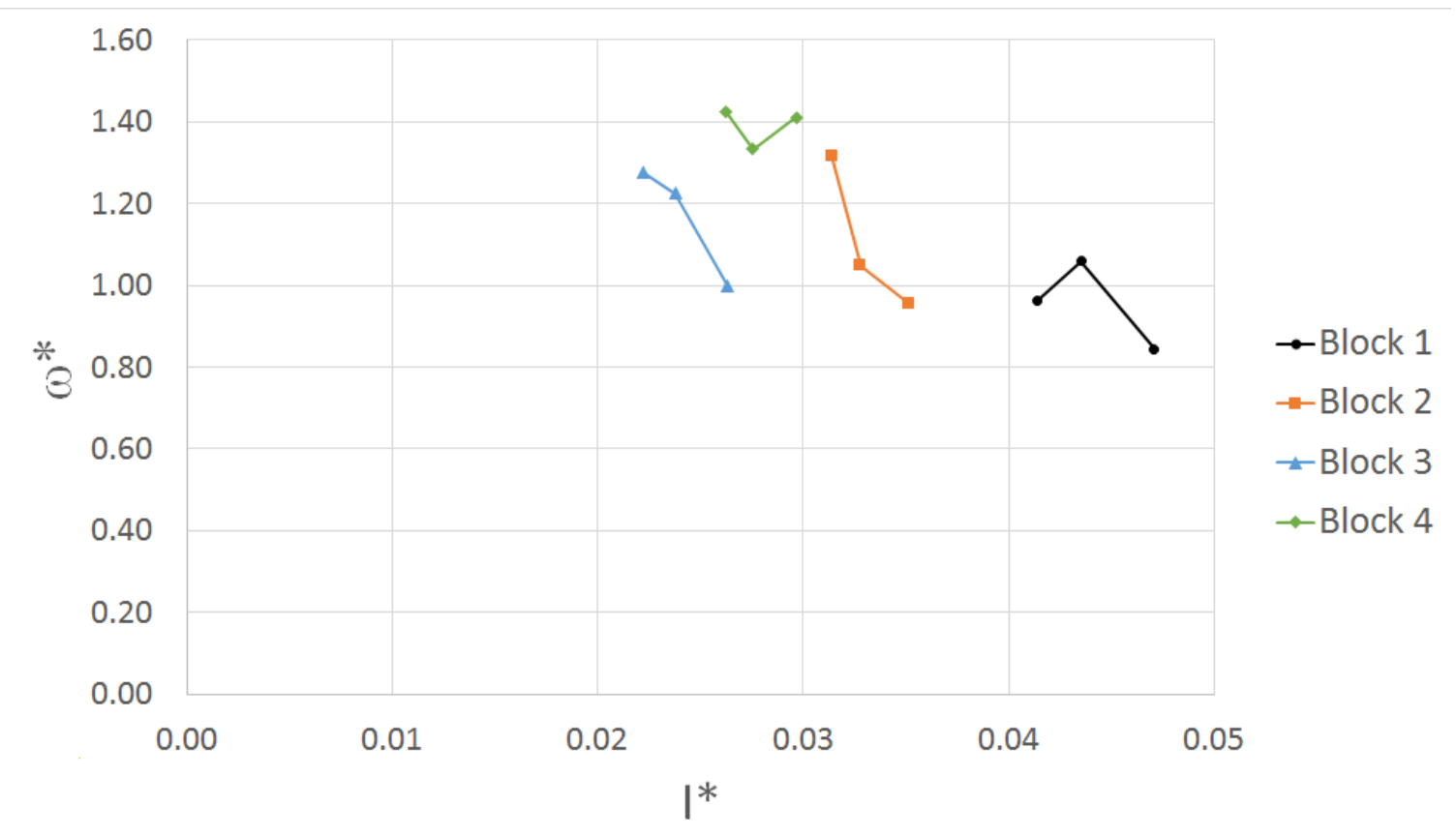

Figure 5.63: Dimension W length scale for Vicon high speed data. 


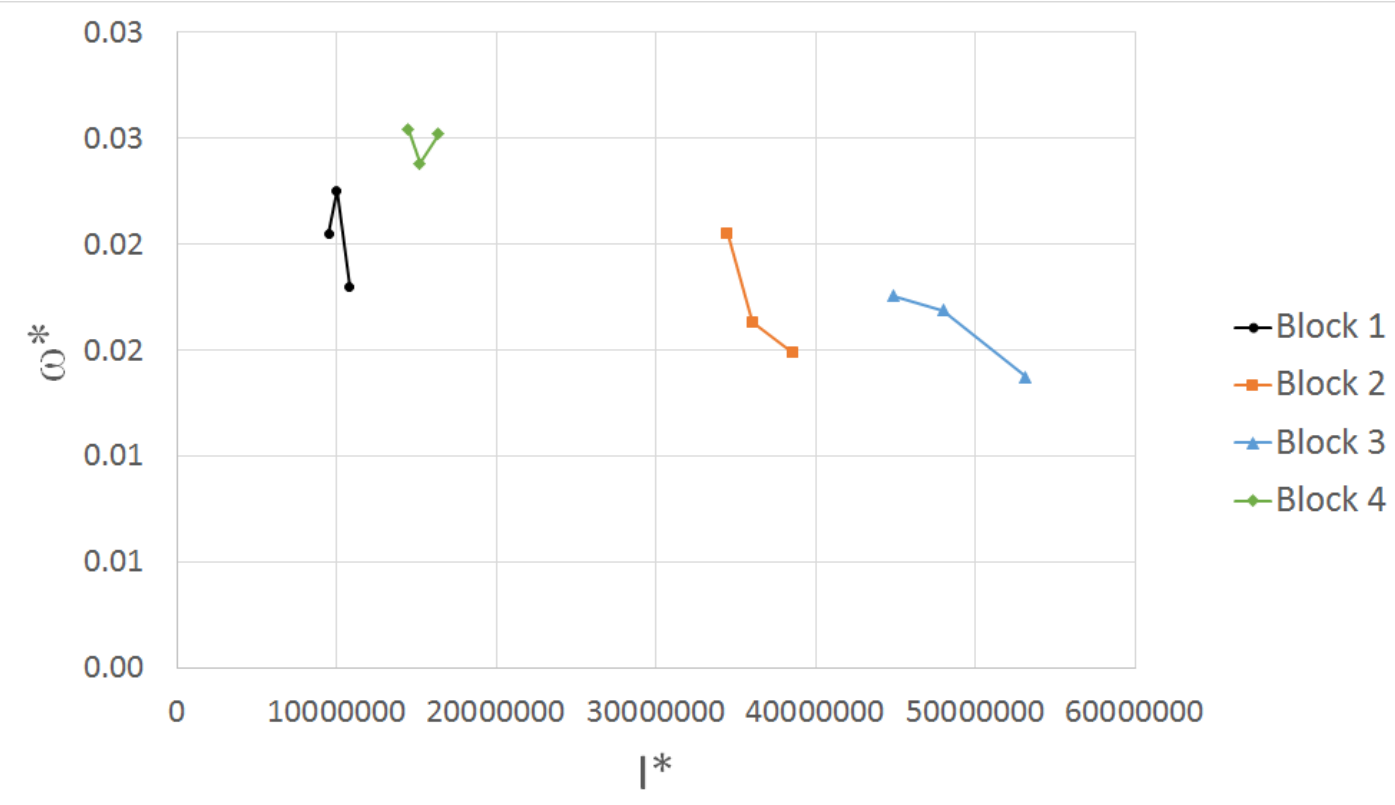

Figure 5.64: Volume/SA length scale for Vicon high speed data.

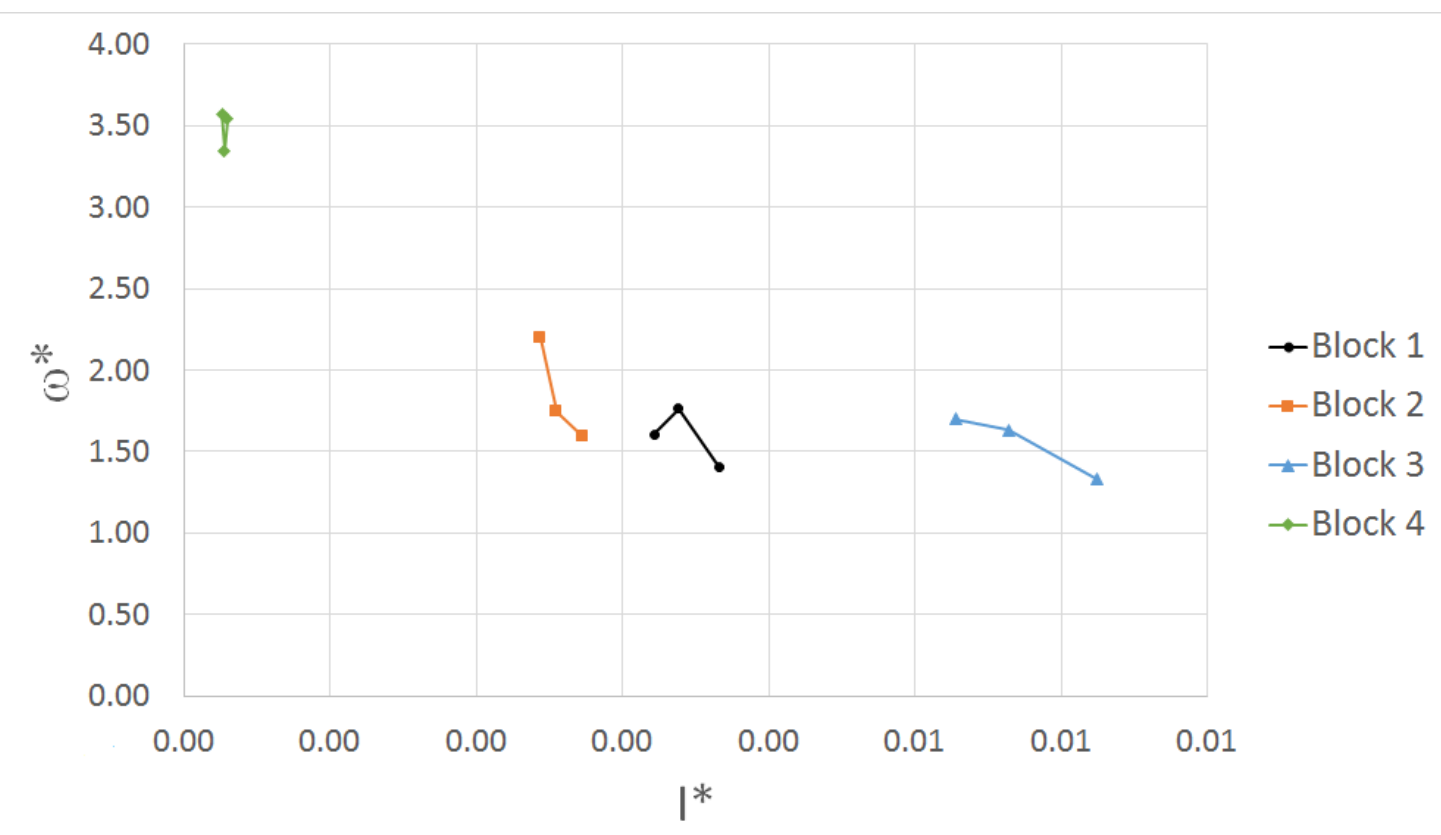

Figure 5.65: Dimension W multiplied by aspect ratio length scale for Vicon high speed data.

An ANOVA analysis performed on the data further examine what factors influenced the data. For the case of Block 4, data was omitted from for the ANOVA analysis on $\omega$ due to the inaccurate data. The ANOVA analysis is still provided for Blockl -3 for $\omega$ and all block sizes for $U x$. 
Table 5-30: Vicon translational velocity data used for ANOVA analysis.

\begin{tabular}{|c|c|c|c|c|}
\hline \multirow{4}{*}{} & \multicolumn{4}{|c|}{$\mathbf{U}_{\mathbf{x}}[\mathbf{c m} / \mathbf{s e c}]$} \\
\cline { 2 - 5 } & \multicolumn{4}{|c|}{ Size } \\
\cline { 2 - 5 } & Block 1 & Block 2 & Block 3 & Block 4 \\
\hline \multirow{3}{*}{ MMOI 1 } & 9.2 & 6.7 & 7.0 & 6.1 \\
\cline { 2 - 5 } & 8.5 & 6.9 & 6.8 & 5.5 \\
\cline { 2 - 5 } & 8.0 & 6.6 & 7.0 & 6.0 \\
\hline \multirow{3}{*}{ MMOI 2 } & 8.1 & 7.1 & 6.9 & 6.1 \\
\cline { 2 - 5 } & 8.1 & 7.0 & 7.0 & 6.2 \\
\cline { 2 - 5 } & 8.7 & 7.1 & 7.1 & 5.8 \\
\hline \multirow{3}{*}{ MMOI 3 } & 9.1 & 6.7 & 7.2 & 5.5 \\
\cline { 2 - 5 } & 8.9 & 6.6 & 7.3 & 5.5 \\
\cline { 2 - 5 } & 9.0 & 7.0 & 7.0 & 5.4 \\
\hline
\end{tabular}

Table 5-31: Vicon rotational velocity data used for ANOVA analysis. Block 4 omitted from ANOVA analysis.

\begin{tabular}{|c|c|c|c|c|}
\hline \multirow{4}{*}{} & \multicolumn{4}{|c|}{ o [rad/sec] } \\
\cline { 2 - 5 } & \multicolumn{4}{|c|}{ Size } \\
\cline { 2 - 5 } & Block 1 & Block 2 & Block 3 & Block 4 \\
\hline \multirow{3}{*}{ MMOI 1 } & 32.3 & 17.7 & 13.5 & 13.7 \\
\cline { 2 - 5 } & 30.7 & 16.9 & 15.2 & 11.4 \\
\cline { 2 - 5 } & 34.2 & 17.7 & 14.7 & 22.0 \\
\hline \multirow{3}{*}{ MMOI 2 } & 34.5 & 15.4 & 14.2 & 13.3 \\
\cline { 2 - 5 } & 32.6 & 13.6 & 13.8 & 22.4 \\
\cline { 2 - 5 } & 36.5 & 14.6 & 14.2 & 19.9 \\
\hline \multirow{3}{*}{ MMOI 3 } & 31.0 & 11.3 & 11.0 & 18.6 \\
\cline { 2 - 5 } & 26.9 & 10.5 & 11.4 & 19.7 \\
\cline { 2 - 5 } & 31.6 & 16.2 & 12.8 & 22.2 \\
\hline
\end{tabular}


Table 5-32: ANOVA analysis for Ux from Vicon data.

\begin{tabular}{lcccccc} 
ANOVA & \multicolumn{1}{c}{ Source of } & & & & & \\
$\quad$ Variation & SS & $d f$ & MS & $F$ & P-value & F crit \\
\hline Sample & 0.05 & 2 & 0.02 & 0.4 & $6.9 \mathrm{E}-01$ & 3.4 \\
Columns & 36.8 & 3 & 12.3 & 197.0 & $4.9 \mathrm{E}-17$ & 3.0 \\
Interaction & 1.5 & 6 & 0.3 & 4.0 & $6.3 \mathrm{E}-03$ & 2.5 \\
Within & 1.5 & 24 & 0.1 & & & \\
& & & & & & \\
Total & 39.9 & 35 & & & & \\
\hline
\end{tabular}

Table 5-33: ANOVA analysis for $\omega$ from Vicon data.

\begin{tabular}{lcccccc} 
ANOVA & \multicolumn{1}{c}{ Source of } & & & & & \\
\multicolumn{1}{c}{ Variation } & SS & $d f$ & MS & $F$ & P-value & F crit \\
\hline Sample & 60.8 & 2 & 30.4 & 10.8 & $8.2 \mathrm{E}-04$ & 3.6 \\
Columns & 1978.5 & 2 & 989.2 & 352.1 & $3.7 \mathrm{E}-15$ & 3.6 \\
Interaction & 19.7 & 4 & 4.9 & 1.8 & $1.8 \mathrm{E}-01$ & 2.9 \\
Within & 50.6 & 18 & 2.8 & & & \\
& & & & & & \\
Total & 2109.5 & 26 & & & & \\
\hline
\end{tabular}

The ANOVA analysis shows that the MMOI did not affect the translational velocity a meaningful amount. However, for the size and interaction, the null hypothesis was rejected. For the case of $\omega$, the null hypothesis was rejected for both Sample and Columns. This indicates that the changing MMOI significantly affects only rotational velocity while changing geometry affects both translational and rotational velocities.

\subsection{Comparison of Test Data}

It was desired to determine a scaling relationship between MMOI and angular velocity of a body using dimensionless terms. All four tests were compared using the most appropriate length scale and average velocity calculations to determine the existence of a scaling relationship. The presence of a scaling relationship will relate the data from each test, regardless of the difference in angular velocities. A comparison of ANOVA data was also completed to quantitatively determine how properties affected translational and rotational velocity data. 
The length scale $S A / L$ seemed to follow the pattern of a power function or logarithmic function. By comparing the data from dimensionless terms calculated using this length scale, a relationship between each type of test was determined. From Figure 5.66, it can be seen that the vertical plane drop tests were the only set of data of the four to scale differently. From observation, the vertical drop tests seemed to be the most susceptible to error. This test was also the only example that analyzed the blocks during acceleration throughout the entire trajectory. This made it difficult to model the velocities. Attempts were made to find an alternative scaling relationship to relate the vertical plane tests to the others using additional dimensionless terms. Tip velocity ratio, Reynolds number, rotational Reynolds number, energy ratio, and dimensionless time were used to create a scaling relationship over all four tests. However, no method was determined to relate vertical plane tests to the other testing methods.

Figure 5.67 shows the data for the horizontal plane test, throwing mechanism analyzed with the Sa5, and throwing mechanism analyzed with Vicon with a power function overlaid. Data from these three testing methods seemed to closely follow the relationship:

$$
\omega^{*}=0.878\left(I^{*}\right)^{-0.227} \quad \text { Equation 5-24 }
$$

The purpose of this study was to find a scaling relationship to relate dimensionless MMOI and dimensionless angular velocity. The relationship in Equation 5-24 can now be used to relate angular velocity and translational velocity to a rigid body with unknown MMOI or a rigid body model manufactured with undesirable MMOI. For example, if a scaled model was manufactured to meet geometric properties, but the MMOI was not able to be matched due to material limitations, testing on the model could be conducted and velocities recorded. Then, equivalent velocities could be calculated for the desired MMOI, to some certainty. The limitation with this method is that only a ratio of angular velocity to translational velocity can be calculated. Since angular and translational velocity are related by the energy transfer between the two, only a value for the ratio between the two can be determined from Equation 5-24. 


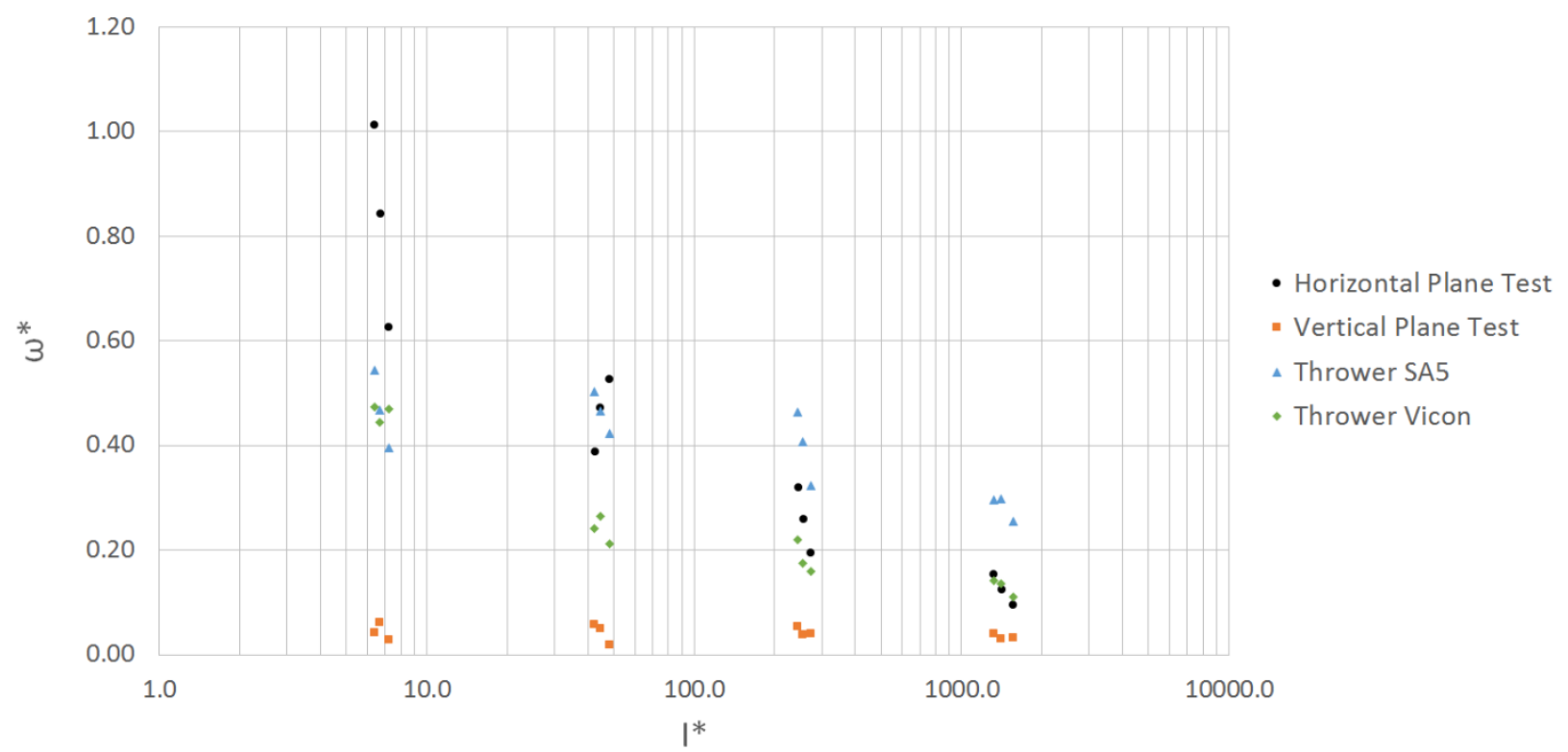

Figure 5.66: Dimensionless term data for the four test methods calculated using the SA/L length scale. Three of the four methods tend to converge to a single line. Demonstrating a scaling relationship.

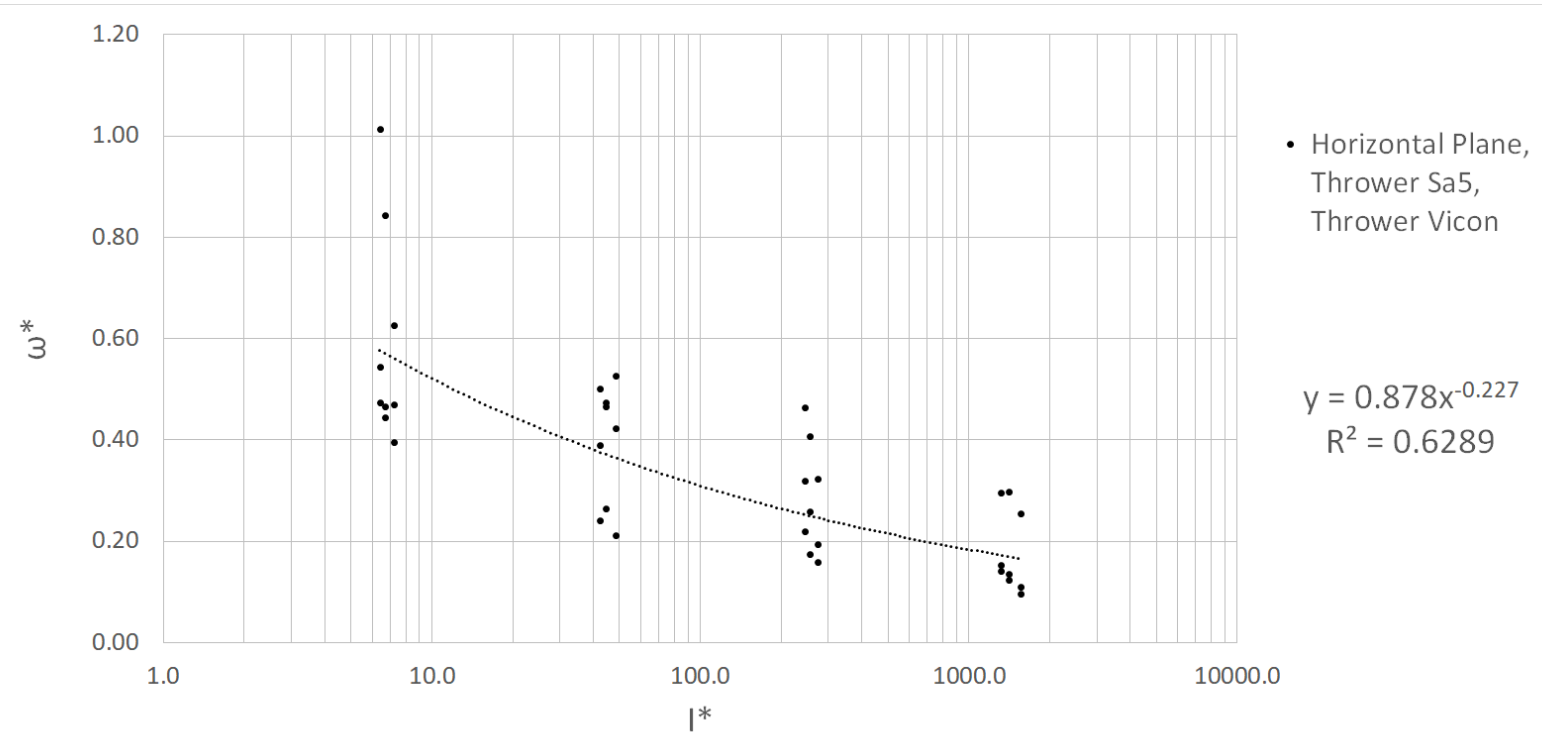

Figure 5.67: Dimensionless data for the horizontal plane test, thrower test using Sa5, and thrower test using Vicon with a power function trendline overlaid.

Figure 5.67 shows that as aspect ratio increases, the range of the dimensionless angular velocity also increases. It is assumed that this is due to the relatively higher drag affecting the velocities. The figure also shows that as dimensionless MMOI decreases, the dimensionless 
angular velocity increases. This follows the idea that a lower MMOI value causes a slower translational velocity and higher angular velocity.

The ANOVA analysis for each test can be seen in Table 5-34 and Table 5-35. For the case of translational velocity, the null hypothesis was always rejected for the geometry of the blocks. Recall that the null hypothesis states that the property does not affect the data. Therefore, the geometry of the blocks was always directly influencing the translational velocity. The same can be said for the rotational velocity. Additionally, the Sample, i.e. changing the MMOI, affected the velocity in some cases, but not all cases. For example, in the horizontal plane testing, changing MMOI of the block did influence the translational velocity but not the rotational velocity. This could be due to the location of the impact of the hammer or other outside factors. It is known from Conservation of Angular Momentum that changing the MMOI of an object increases its angular velocity while influenced by the same force. The large effects of geometry on the velocities relative to changing MMOI is assumed to be due to the increase in drag as the block size increased.

Since the throwing mechanism was analyzed with the SA5 high speed camera as well as the VICON system, the ANOVA analysis should be the same. However, the values do not match. This could be due to the increased range over which the trajectories were tracked using the VICON system. Additionally, the throwing mechanism itself may have been releasing the blocks at a slightly different position. Regardless, it is important to note that these values should show similar results. 
Table 5-34: Combined ANOVA analysis for Ux for each of the four testing methods.

\begin{tabular}{|c|c|c|c|c|c|c|}
\hline \multirow{2}{*}{} & \multicolumn{6}{|c|}{ Combined ANOVA U. } \\
\cline { 2 - 7 } & \multicolumn{3}{|c|}{ Vertical Plane } & \multicolumn{3}{c|}{ Horizontal Plane } \\
\cline { 2 - 8 } & $F$ & $F$ crit & $\begin{array}{l}\text { Reject Null } \\
\text { Hypothesis }\end{array}$ & $F$ & $F$ crit & $\begin{array}{l}\text { Reject Null } \\
\text { Hypothesis }\end{array}$ \\
\hline Sample (MMOI) & 7.4 & 3.4 & YES & 0.2 & 3.4 & NO \\
\hline Columns (Geometry) & 11.9 & 3.0 & YES & 65.0 & 3.0 & YES \\
\hline Interaction & 3.8 & 2.5 & YES & 2.0 & 2.5 & NO \\
\hline & \multicolumn{2}{|c|}{ Throwing Mechanism SA5 } & \multicolumn{2}{|c|}{ Throwing Mechanism Vicon } \\
\cline { 2 - 8 } & $F$ & $F$ crit & $\begin{array}{l}\text { Reject Null } \\
\text { Hypothesis }\end{array}$ & $F$ & $F$ crit & $\begin{array}{l}\text { Reject Null } \\
\text { Hypothesis }\end{array}$ \\
\hline Sample (MMOI) & 2.2 & 3.4 & NO & 0.4 & 3.4 & NO \\
\hline Columns (Geometry) & 234.8 & 3.0 & YES & 197.0 & 3.0 & YES \\
\hline Interaction & 1.2 & 2.5 & NO & 4.0 & 2.5 & YES \\
\hline
\end{tabular}

Table 5-35: Combined ANOVA analysis for $\omega$ for each of the four testing methods.

\begin{tabular}{|c|c|c|c|c|c|c|}
\hline \multirow{2}{*}{} & \multicolumn{7}{|c|}{ Combined ANOVA $\boldsymbol{~}$} \\
\cline { 2 - 8 } & \multicolumn{3}{|c|}{ Vertical Plane } & \multicolumn{3}{c|}{ Horizontal Plane } \\
\cline { 2 - 8 } & $F$ & $F$ crit & $\begin{array}{l}\text { Reject Null } \\
\text { Hypothesis }\end{array}$ & $F$ & $F$ crit & $\begin{array}{l}\text { Reject Null } \\
\text { Hypothesis }\end{array}$ \\
\hline Sample (MMOI) & 1.4 & 3.4 & NO & 17.5 & 3.4 & YES \\
\hline Columns (Geometry) & 637.2 & 3.0 & YES & 16.8 & 3.0 & YES \\
\hline Interaction & 28.7 & 2.5 & YES & 6.4 & 2.5 & YES \\
\hline & \multicolumn{2}{|c|}{ Throwing Mechanism SA5 } & Throwing Mechanism Vicon \\
\cline { 2 - 8 } & $F$ & $F$ crit & $\begin{array}{l}\text { Reject Null } \\
\text { Hypothesis }\end{array}$ & $F$ & $F$ crit & $\begin{array}{l}\text { Reject Null } \\
\text { Hypothesis }\end{array}$ \\
\hline Sample (MMOI) & 44.8 & 3.4 & YES & 10.8 & 3.6 & YES \\
\hline Columns (Geometry) & 527.8 & 3.0 & YES & 352.1 & 3.6 & YES \\
\hline Interaction & 3.2 & 2.5 & YES & 1.8 & 2.9 & NO \\
\hline
\end{tabular}




\section{Chapter 6 Conclusions and Recommendations}

For the horizontal plane test and two throwing mechanism tests, a relationship between dimensionless MMOI and dimensionless angular velocity was determined using the length scale $S A / L$. The relationship between the dimensionless terms is governed by the equation:

$$
\omega^{*}=0.878\left(I^{*}\right)^{-0.227}
$$

From this relationship, a ratio of angular velocity and translational velocity can be calculated for a rigid body with known MMOI and geometric dimensions. The test articles examined were simple shapes. That is, the geometries were based on symmetric shapes with relatively minimal aerodynamic forces to influence results. Increased velocities and complex geometries introduce more probability for outside influence on the trajectory of the test articles.

An example of this method for application is shows as follows:

A team of researchers are analyzing a scaled ridged body. Due to manufacturing limitations, the MMOI of the scaled model is 1,000,000 $\mathrm{g}-\mathrm{cm}^{2}$ instead of the target MMOI of 900,000 $\mathrm{g}_{-\mathrm{cm}^{2}}$. The model has a Surface Area/Length ratio of $12 \mathrm{~cm}$ and the density of the model material is recorded as $0.94 \mathrm{~g} / \mathrm{cm}^{3}$. The model is fired downrange and an average translational velocity of $800 \mathrm{~cm} / \mathrm{sec}$ and angular velocity of $30 \mathrm{rad} / \mathrm{sec}$ are recorded. For these properties, $I^{*}$ $=4.3$ and $\omega^{*}=0.45$. Figure 6.1 shows this point, symbolized by the red $X$, plotted with the other values. 


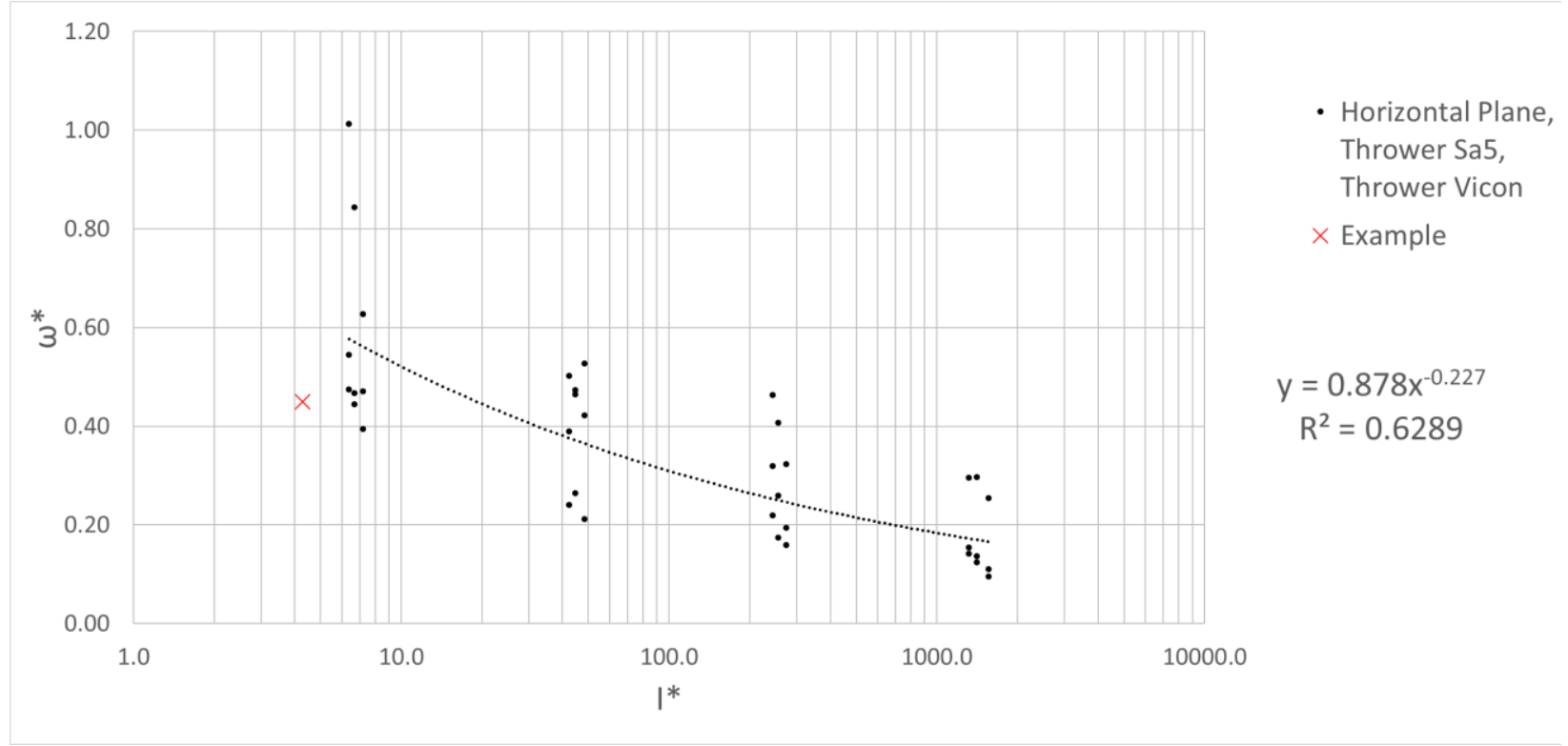

Figure 6.1: Application Example

Using the derived equation, the target value for $I^{*}$ is used to calculate what the actual $\omega$ should be.

$$
\omega^{*}=\frac{\omega(12)}{u}=0.878(3.85)^{-0.227}
$$

Simplifying, the velocity ratio becomes

$$
\frac{\omega}{u}=0.05 \frac{\mathrm{rad}}{\mathrm{cm}}
$$

From the velocity ratio, the translational velocity can be held constant from the experimental data, and the new $\omega$ is calculated to be $43 \mathrm{rad} / \mathrm{sec}$.

Another relationship between the velocity ratio and a dimensionless term independent of either velocity would give a more accurate solution. However, this method gives a close approximation for what the angular velocity would be for the desired MMOI value.

The ANOVA analysis also provided insight into how block properties affected the velocities. According to data collected, when creating a scale model UAS with the option to match either MMOI or geometry, it is more favorable to match geometry. Geometry of the model has a significantly higher effect on the angular velocity when compared to the MMOI. As for translational velocity, each factor has a similar effect on the system that is significantly small when 
compared to effects on angular velocity. Alternatively, the ANOVA analysis does not account for the increase in MMOI from increasing block size. Therefore, it is impossible to say with certainty that the geometry of the block affects the data rather than the increase in MMOI between sizes. To determine if geometry alone affects the data, MMOI of the block would need to be held constant with the geometry was changed. Refer to Table 5-34 and Table 5-35 for specific ANAVA values.

Further analysis using more samples would give a larger range over which to compare data. By placing the additional weights in unsymmetrical locations about the CG, more MMOI data points could have produced. This would have also allowed the study to include effects when the CG does not coincide with the CM.

Additionally, block material should be carefully selected for durability to increase repeatability. Although the foam blocks used for this experiment were handled with care, small inclusions and other surface defects surely added to the error within the experiment. The ability of the material to flex also introduced the influence of impulse on the blocks. Although the impact during the horizontal plane tests was assumed to be perfectly inelastic, it was possible that increased impulse as the additional masses were moved away from the CG could have increased the relative energy transferred from the hammer to the block.

A more consistent firing mechanism was also a leading factor for reducing error. The firing mechanism, in general, could have comprised of better manufactured parts to reduce friction losses. For the throwing mechanism, it was difficult to grip the larger blocks before launch. It is recommended to use additional smaller test articles or modify the holding mechanism on the arm to accept larger block sizes. Other methods to study relative rotation and translation, such as pneumatic jets, a wind tunnel, or an upgraded pendulum, could also be used. Testing in different mediums is also recommended to increase the range of velocities tested. Testing in water would require a denser material to reduce buoyancy effects. Testing in a vacuum would remove drag from influencing the block's trajectory.

Testing in the vertical plane was used to recreate results from the horizontal table, as well as draw comparisons with previous data. However, no previous data experimented with rotation about the z-axis. The two methods of analyzing the throwing mechanism were used to compare results of the two systems. The four tests examined a range of translational velocities from approximately $130 \mathrm{~cm} / \mathrm{sec}$ to $900 \mathrm{~cm} / \mathrm{sec}$ and angular velocities from approximately $0.5 \mathrm{rad} / \mathrm{sec}$ to 
$36 \mathrm{rad} / \mathrm{sec}$. However, the vertical plane tests were omitted from the scaling analysis due to their inconsistent behavior and tendency to tumble out of plane.

Factors such as block unbalanced weights made some tests inconsistent. The onset of tumbling could have been influenced by imperfections and imbalance in the block rather than MMOI and angular velocity alone. Balance of the additional masses in the blocks was not an important factor in horizontal testing, but in testing in the vertical plane and testing with the thrower, it had enough influence to affect the data. Future experiments should take care to machine weights with high tolerance and consistently balance each point mass in each plane of the block. 


\section{Chapter 7 References}

[1] F. Capra, Learning from Leonardo: Decoding the Notebooks of a Genius, San Francisco: Berrett-Koehler, 2013.

[2] S. G. Rock and e. al., "Validation of an Automated Chimera Methodology for Aircraft Escape Systems Analysis," American Instute of Aeronautics and Astronautics, Inc., 1998.

[3] M. Elson and S. J. Lingard, "Object Orientated Ejection Sear Model," American Institute of Aeronautics and Astronautics, Inc., 1997.

[4] L. A. Obergefell and I. Kaleps, "Simulatin of Body Motion During Aircraft Ejection," in Mathematical Modeling, Great Britain, 1988.

[5] B. Saranjam, "Experimental and Numerical Investigation of an Unsteady Supercavitating Moving Body," Ocean Engineering, vol. 59, pp. 9-14, 2013.

[6] A. Andersen, U. Pesavento and Z. J. Wang, "Unsteady aerodynamics of fluttering and tumbling plates," Journal of Fluid Mechanics, vol. 541, pp. 65-90, 2005.

[7] A. Andersen, U. Pesavento and Z. J. Wang, "Analysis of transitions between fluttering, tumbling and steady descent of falling cards," Journal of Fluid Mechanics, vol. 541, pp. 91-104, 2005.

[8] C. Jin and K. Xu, "Numerical study of the unsteady aerodynamics of freely falling plates," Comm. Comp. Phys, vol. 3, pp. 834-851, 2008.

[9] W. W. Willmarth, N. E. Hawk and R. L. Harvey, "Steady and unsteady motions and wakes of freely falling disks," Physics of Fluids, vol. 7, p. 197, 1964.

[10] Vicon Motion Systems, "Vicon Documentation," Vicon Motion Systems, [Online]. Available: https://docs.vicon.com/display/Tracker33/Camera+calibration+feedback. [Accessed Feburary 2018].

[11] S. Biringen and C.-Y. Chow, An Introduction to Computational Fluid Mechanics by Example, Wiley, 2011.

[12] P. R. Payne, "The Virtual Mass of a Rectangular Flat Plate of Finite Aspect Ratio," Hydronautics, vol. 8, no. 5, pp. 221-257, 1981.

[13] C. D. Griffin, Vicon Software Quaternion and Position Data Conversion to Body Rates, Morgantown: MATLAB Central File Exchange, 2017.

[14] J. K. Borchardt, "Unmanned aerial vehicles spur composites use," Reinforced Plastics, vol. 48, pp. 28-31, 2004. 
[15] R. Austin, Unmanned Aircraft Systems, John Whiley and Sons, 2010.

[16] L. Mahadevan, W. S. Ryu and A. D. T. Samuel, "Tumbling cards," Physics of Fluids (1994-present), vol. 11, pp. 1-3, 1999.

[17] J. D. Iversen, "Autorotating flat-plate wings: the effect of the moment of inertia, geometry and Reynolds number," Journal of Fluid Mechanics, vol. 92, pp. 327-348, 1979.

[18] S. Bönisch and V. Heuveline, "On the numerical simulation of the unsteady free fall of a solid in a fluid: I. The Newtonian case," Computers $\backslash \&$ fluids, vol. 36, pp. 1434-1445, 2007.

[19] A. Belmonte, H. Eisenberg and E. Moses, "From Flutter to Tumble: Inertial Drag and Froude Similarity in Falling Paper," Phys. Rev. Lett., vol. 81, no. 2, pp. 345-348, 71998.

[20] S. B. Field, M. Klaus, M. G. Moore and F. Nori, "Chaotic dynamics of falling disks," Nature, vol. 388, p. 40817, 71997.

[21] H. Guan, S. Aoki, K. Ejiri and others, "A new method for correcting geometric distortion in digital images and its application to panorama image composition," Ricoh Technical Report No. 23, 1997.

[22] M. Lennie, J. Wendler, G. Pechlivanoglou, C. Nayeri, C. O. Paschereit and D. Greenblatt, "Development of a Partially Stochastic Unsteady Aerodynamics Model," in 35th Wind Energy Symposium, 2017.

[23] H. J. Lugt, "Autorotation," Annual Review of Fluid Mechanics, vol. 15, pp. 123-147, 1983.

[24] R. Mittal, V. Seshadri and H. S. Udaykumar, "Flutter, Tumble and Vortex Induced Autorotation," Theoretical and Computational Fluid Dynamics, vol. 17, pp. 165-170, 014 2004.

[25] N. Zorzi, J. M. C. Pereira and J. C. F. Pereira, "On the uncertainty quantification of the unsteady aerodynamics of 2D free falling plates," Theoretical and Computational Fluid Dynamics, vol. 29, pp. 329-341, 0182015.

[26] P. Ern, F. Risso, D. Fabre and J. Magnaudet, "Wake-Induced Oscillatory Paths of Bodies Freely Rising or Falling in Fluids," Annual Review of Fluid Mechanics, vol. 44, pp. 97-121, 2012.

[27] A. J. DuBois, in The Mechanics of Engineering, New York, John Wiley \& Sons, 1908, pp. 363-364.

[28] C. Hecker, "Rigid Body Dynamics," March 1997. [Online]. Available: http://chrishecker.com/images/e/e7/Gdmphys3.pdf. [Accessed 2017]. 
[29] P. Dupleich, "Rotation in Free Fall of Rectangular Wings of Elongated Shape," National Advisory Committee for Aeronautics, 1949.

[30] Y. K. a. O. Mochizuki, "Role on Moment of Inertia and Vortex Dynamics for a Thin Rotating Plate," World Journal of Mechanics, 2013.

[31] B \& H Foto \& Electronics Corp., Mavic Pro Model UAV.

[32] K. Varshney, S. Chang and Z. J. Wang, "Unsteady aerodynamic forces and torques on falling parallelograms in coupled tumbling-helical motions," Physical Review E, vol. 87, no. 5, p. 053021, 2013. 


\section{Appendix A \\ Sample MATLAB Code for Camera Calibration App}

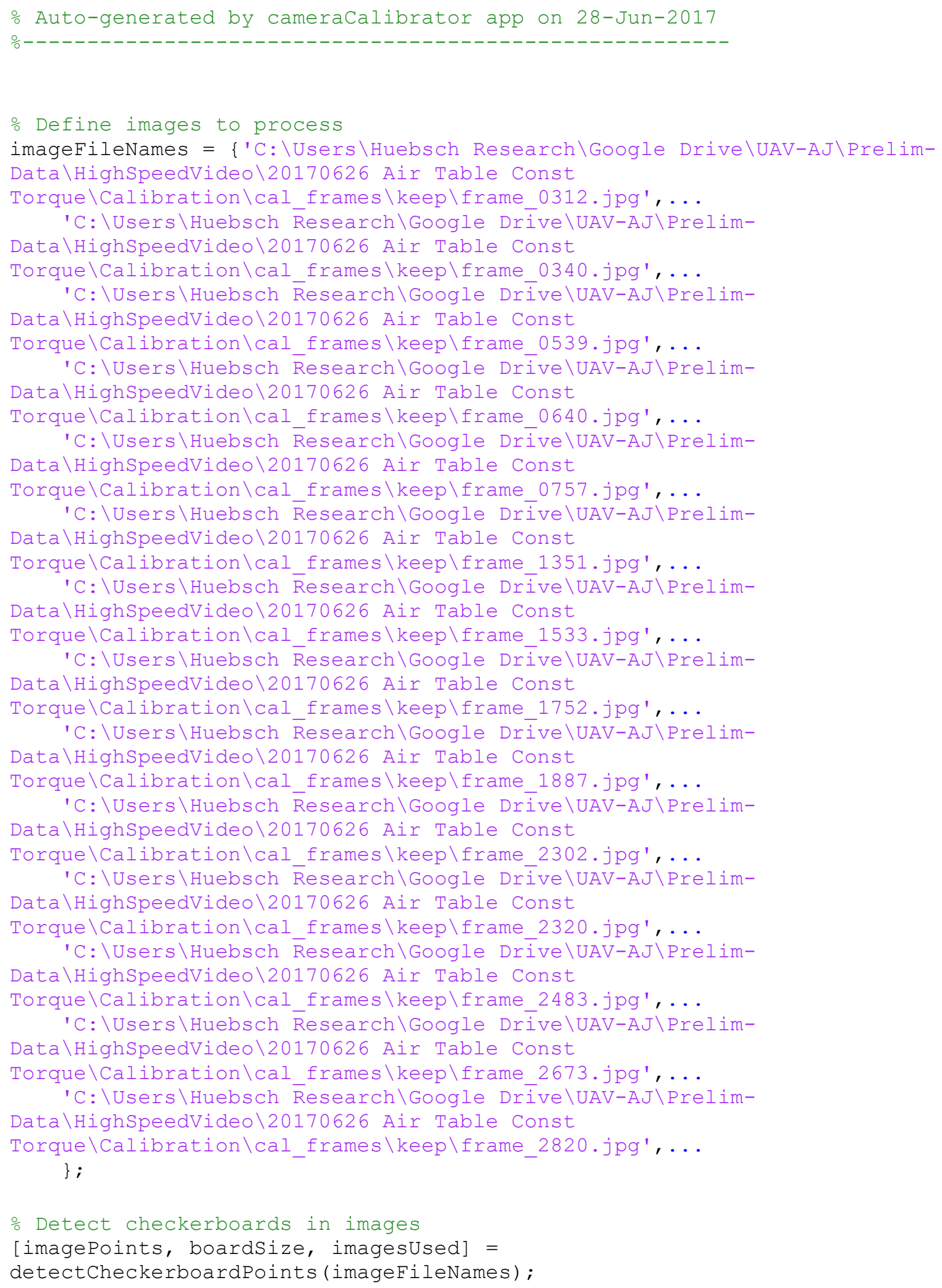




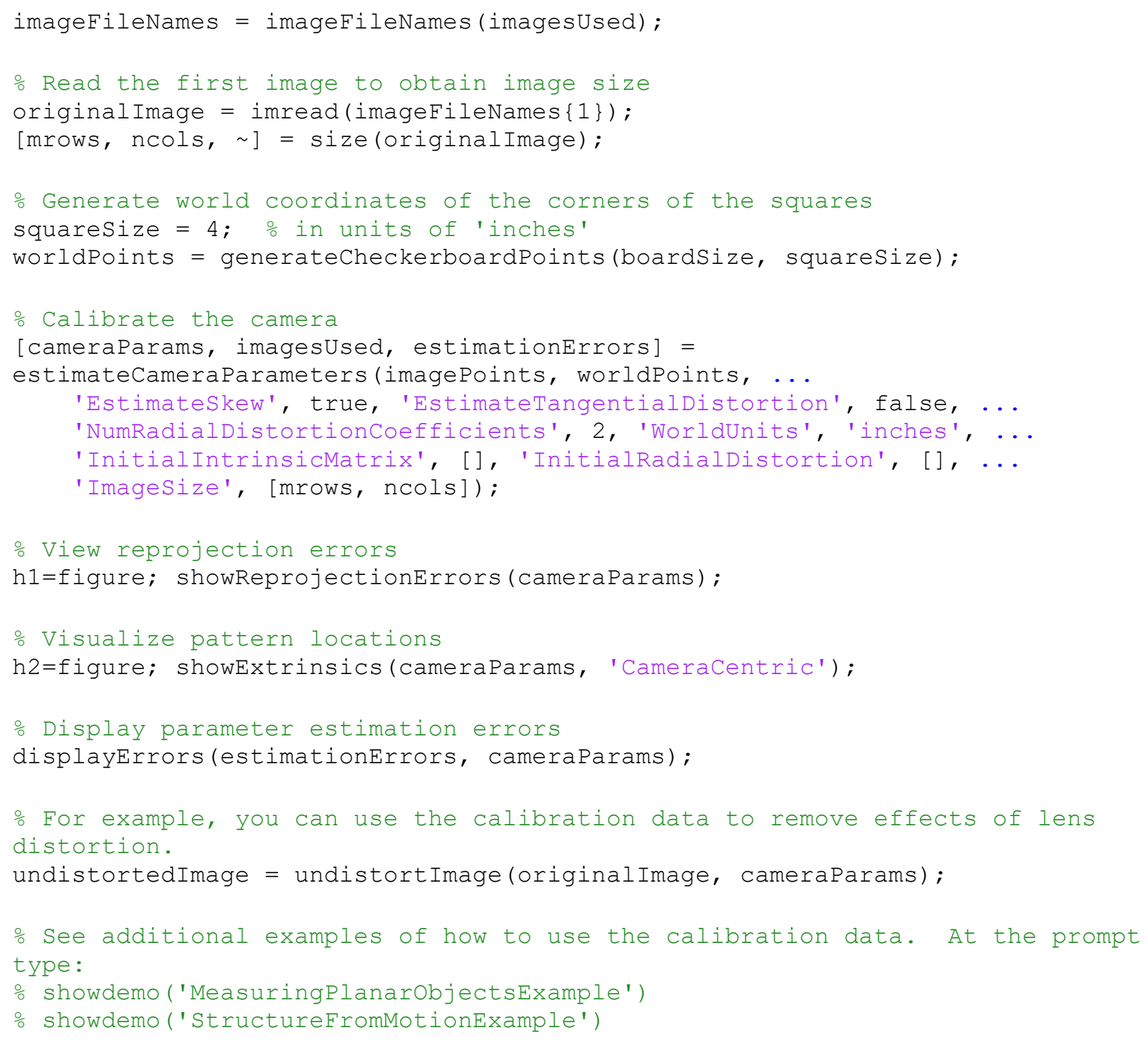




\section{Appendix B}

Sample MATLAB Code for converting Vicon recorded quaternion data to body rates. This code imports a .csv file from Vicon software and converts quaternion data to more applicable plots and data. Code provided by Griffin [13], and slightly modified to smooth data for analysis. [13]

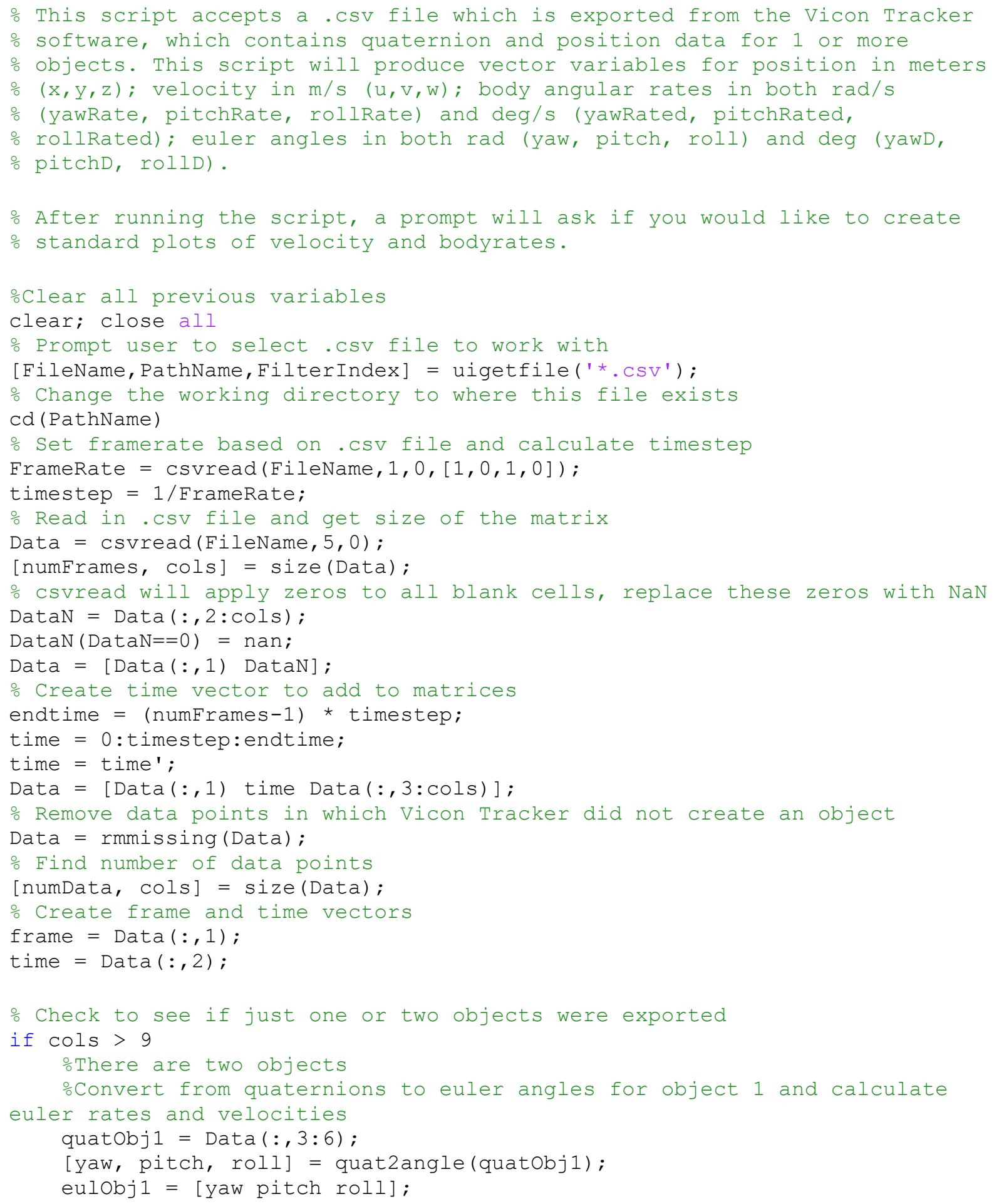




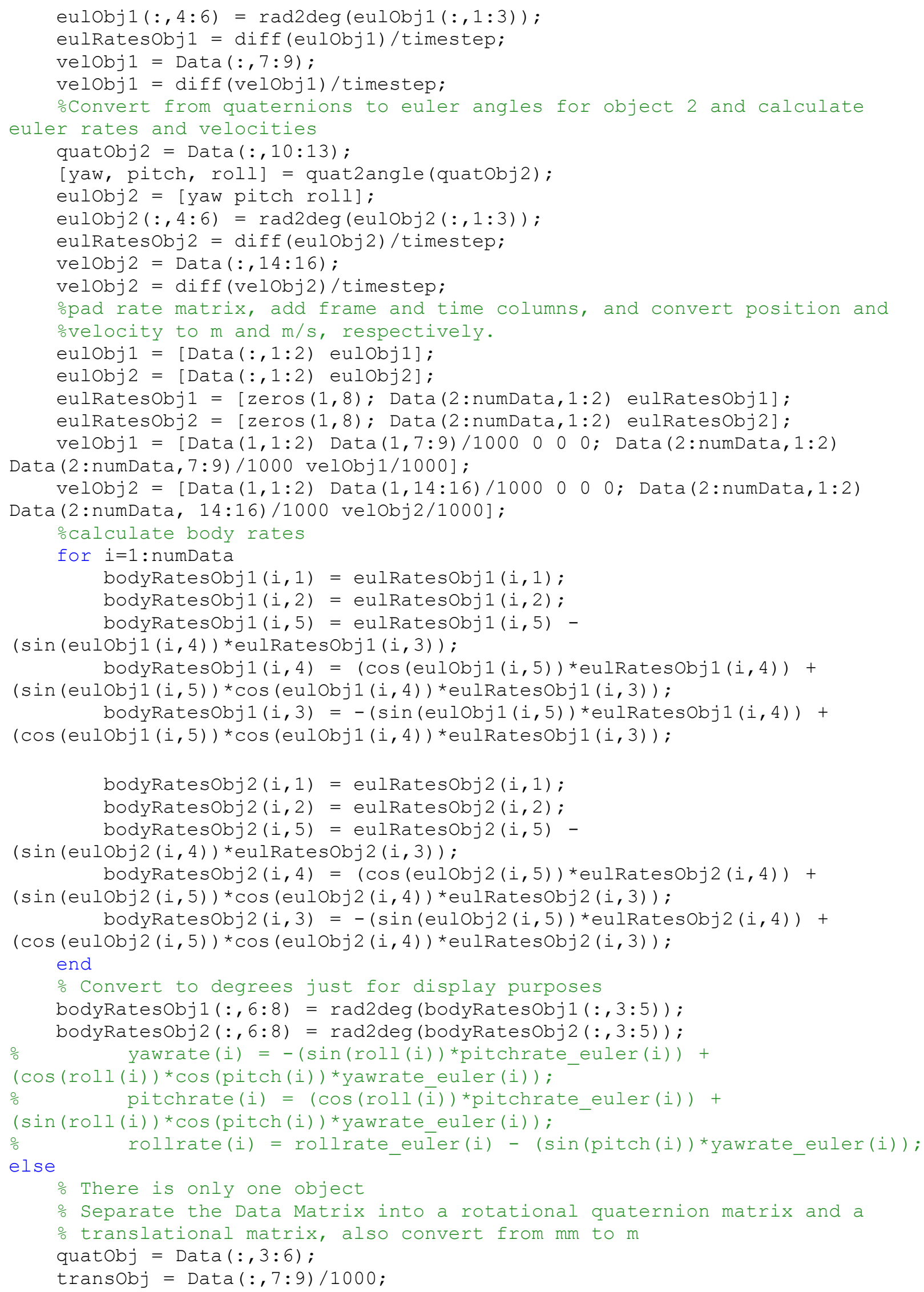




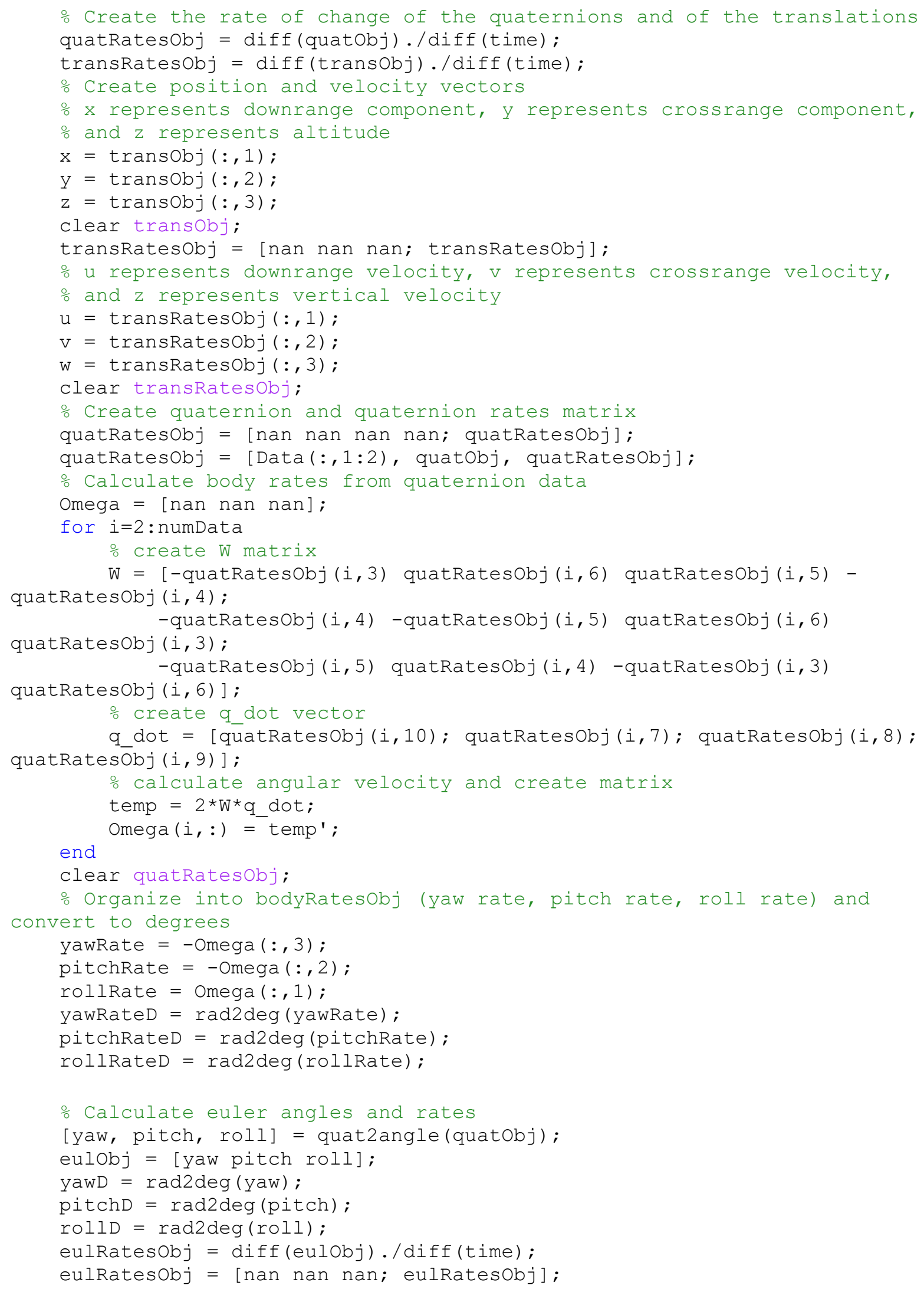




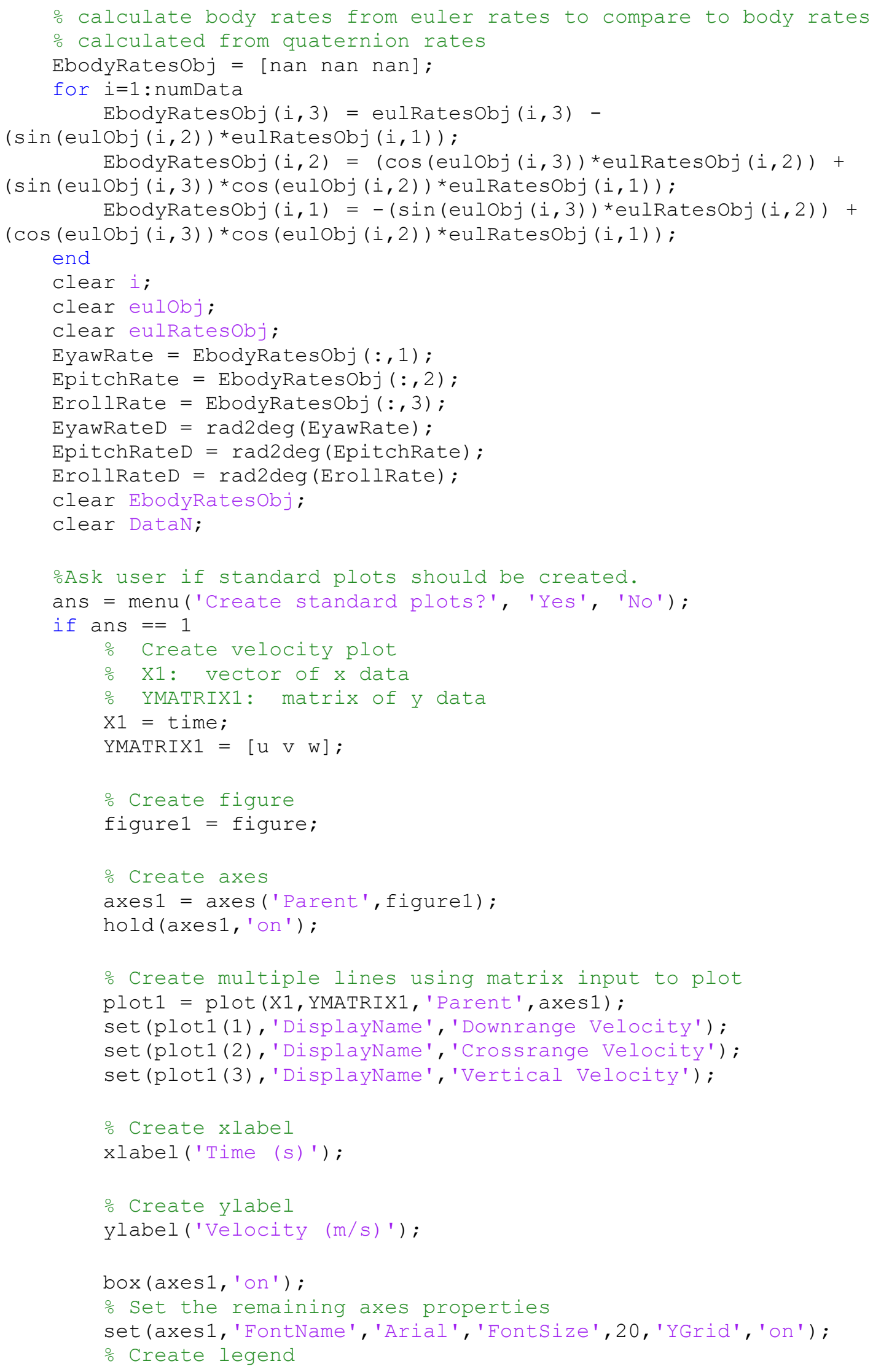




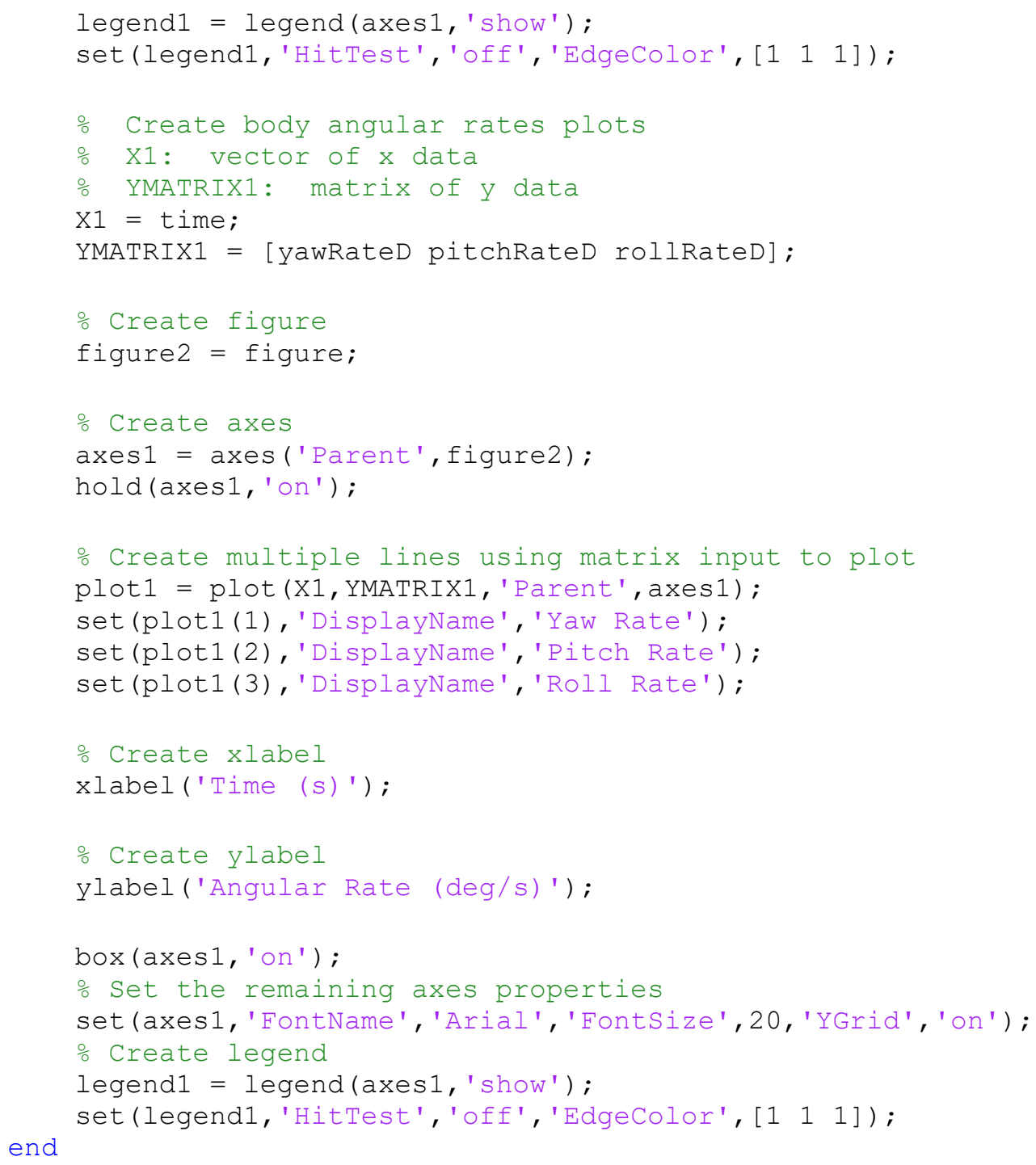


figure ()

plot (X1,pitchRateD)

xlabel ('time [sec]')

ylabel ('pitch rate [deg/sec]')

hold on

plot (X1, Ps)

plot (X1 (idx2), val2,' *k', 'MarkerSize', 7) 